\title{
Am I Luckier Than You? Individual Differences in Precognition Performance
}

\author{
Marco Zdrenka
}

A thesis submitted to Victoria University of Wellington in fulfilment of the requirements for the degree of Doctor of Philosophy in Psychology

Victoria University of Wellington 2018 


\section{ABSTRACT}

The results found in parapsychological research have not been particularly persuasive, in large part due to a lack of replicability of those studies purporting evidence in support of the existence of psi phenomena. I propose that a more promising avenue of research into psi focuses on the potential correlates of psi performance (i.e. factors that correlate with above- or below-chance performance on luck-based tasks without any known causal mechanism). Specifically, individual differences, such as belief in ESP or extraversion, have been shown to correlate with psi performance in forcedchoice precognition experiments (where participants predict a future chanceevent by choosing one option from a limited number of options). Thus, the goal of this thesis was to synthesise existing literature on predictors of psi performance, to identify the best predictors of psi performance, and to test these predictors using the latest experimental paradigms while also examining its external validity in the real world.

My first study (Study I) was a never-before-conducted meta-analysis of all research that has looked at individual differences and psi performance in forced-choice precognition experiments. Overall, 57 studies published between 1945 and 2016, including 35 individual difference measures, were subject to meta-analysis (Zdrenka \& Wilson, 2017). Six individual difference measures, namely, luck belief (the belief that luck is primarily controllable), perceptual defensiveness, openness to experience, belief in ESP, extraversion, and time belief as dynamic, were found to significantly correlate with psi performance.

Study II attempted to examine these promising individual difference variables using one of Bem's (2011a) previously successful experimental paradigms, while also using Bem's own materials and resources. Thus, Study II is a replication of the first of his experiments, Precognitive Detection of Erotic Stimuli, which was shown to be the most robust of the studies reported by Bem (Bem, Tressoldi, Rabeyron, \& Duggan, 2015). In summary, Study II explored precognition using this most robust experimental paradigm, in combination 
with the most promising individual difference correlates identified in Study I. Belief in ESP was found to significantly predict psi performance (i.e. a 'sheepgoat' effect; Schmeidler, 1943, 1945) whereas the other individual differences did not.

The aims of Studies III(A) and III(B) were to investigate the utility of paranormal belief as a predictor of performance in two real-life lottery studies. In the first Study III(A), a large sample of New Zealanders completed a measure of paranormal belief and provided their preferred lotto numbers. Paranormal belief (and a range of other lottery behaviours) failed to predict lottery success. Finally, a pilot study is presented (Study III(B)) incorporating the same individual difference measures from Study I and II, but applied in a real-world setting. We examined participants' predictions of numbers drawn in the New Zealand lottery over eight weeks, as the lottery presents a real-world example of forced-choice precognition (in that the future event is ostensibly unpredictable and there are a limited number of choices to pick from, i.e. the numbers 1-40). However, there was a low rate of participation that was caused, in part, by the time-commitment required, and therefore the study was underpowered and the results inconclusive. Limitations were also identified by potential preference biases distorting the results (due to there being only a single target set per draw, i.e. the winning lotto number set).

Overall, this thesis attempted to answer two main research questions: (1) which individual difference measures are the most predictive of psi performance in the forced-choice precognition domain? (Studies I) and, (2) are these individual difference predictors consistent and reliable across a range of settings? (Studies II through III(B)). I found partial support for a significant predictor, in that Belief in ESP significantly correlated with psi performance in a forced-choice precognition replicatory experiment, but further research is necessary to confirm whether this finding - based on experimental laboratorybased studies-translates to real-world psi, and to address the limitations found in Studies II and III. An alternative interpretation is that the results are due to random variation—given that they are weak and inconsistent—and psi does not 
exist. Pre-registered studies and prospective meta-analyses are likely to determine which interpretation prevails in the end. 


\section{ACKNOWLEDGEMENTS}

It wouldn't be a thesis about luck, without first acknowledging the large role that 'luck' played in getting me to where I am today. While hard work does pay off, I am extremely fortunate that the stars aligned from day one (when the universe first began) such that I was able to think, write, and meet all the people that helped me along the way.

It is fitting, then, to start with Marc Wilson, my supervisor, who happened to accept me as his PhD student even though we had never met (and he did not know that I was interested in parapsychology!). Perhaps it was precognition. It was only after our first meeting together that I decided to bare my soul and let him know that my 'other' interest was this phenomena known as 'psi'. I was pleasantly surprised (or perhaps more shocked) to find out that not only was he well versed in the parapsychological literature, but was happy to supervise me on that topic if I wanted to pursue it. The rest is history. I'm truly thankful for how supportive you've been over these past three years (you always know how to put someone at ease). You provided just the right amount of guidance to get me to the end, but were hands-off enough that I could always go in a direction I enjoyed. Thank you.

Three academic mentors also led me down this path. The first is Kumar Yogeeswaran, who re-invigorated my interest in psychology after I took his Social Psychology class. I wouldn't have considered postgrad if it wasn't for you. You're a great mentor and a great teacher, and made my honours year the best it could be. The second is Caroline Watt. Once Marc gave me the green light to pursue parapsychology, I delved into the literature but soon found the field to be very polarised. Most papers were either pro-psi or anti-psi, and there didn't seem to be much of a middle ground (or at least, an interpretation of the core data that both sides accepted). As 'luck' would have it, I came across Caroline's online parapsychology course, which provided a balanced overview of all sides of the debate that I could find nowhere else. It provided me with some structure amidst the chaos, and I am grateful to you for that. And finally Daryl 
Bem, thank you for graciously providing me with your replication package (which enabled me to conduct Study II). I can say without hesitation that you are a solid figure to look up to in both the field of psychology and parapsychology.

Next-I wouldn't be here without my family. Dad, for always helping me out when I needed it. Mum, for being extraordinarily proud of my academic achievements. Both of you didn't think twice about the fact that I chose to study even more (I had already spent 6 years at university before pursuing my $\mathrm{PhD}$ )-you were just supportive in whatever I wanted to do. It really helped put me at ease with my decision. My brother Paul, you were always there to keep things light-hearted, bringing up X-files references whenever my research topic came up. You made me step back and laugh, and I appreciate that. My sister Helene, you were always so inquisitive about my work and provided lots of sage advice along the way. I am grateful to have such a caring sister.

Also to meine Oma, Chai, Elaine, Annie, Marwan, Jenny, Ceci, Nox, Grace, and Nikolai, you've all been a great support for me and I'm glad to have you all as part of my extended family.

To all of my friends, thanks for listening to me ramble about what 'luck' is and whether probabilities exist. I will have no more excuses not to play some board games now and actually leave my house (I'm sure the Vitamin D won't hurt either).

Last but not least is my partner Jennifer, who often jokes that this $\mathrm{PhD}$ is practically hers-but she's not far off from the truth. There is no way I could have done this without her support, encouragement, and patience. You made all of this worth it, and I hope I can do the same for you in your future endeavours. 


\section{PREFACE}

From a young age, I have been drawn to weird or controversial topics. I remember reading books about the Bermuda Triangle, aliens, conspiracies, and everything in between. It was always difficult to decipher fact from fiction, due to the sensationalism you often find in these subjects. What I didn't know at the time, however, was that a field of research existed that attempted to examine [some of] these concepts scientifically.

This piqued my interest, as I wanted to learn about these topics in an objective way. Fringe topics never interested me for the sake of it being fantastical, or because I have a creative mind (which I don't). Rather, it was more about truth for me. I wanted to know exactly how the world operated, and assumed that since there were still so many unknowns in this world, fact must be stranger than fiction (i.e. the objective truth of these unknowns).

I think it was Bem's (2011a) paper that opened my eyes up to the world of parapsychology, and I was fascinated by its potential implications. If psi were real, were some people altering the laws of probability unbeknownst to those around them? Can the future really influence the present? It was not long after having these thoughts swirling around my head that I met Marc and told him of my newfound interest (but not before a discussion of some rather more conventional research questions, to test the waters first!), and the topic of this thesis was born.

While it might have been rather ambitious, the structure of the thesis was specifically designed to give me the best chance of finding out the truth or falsity of psi. Therefore, the subject matter addressed in my experiments was not necessarily the most interesting (e.g. I'd rather have pursued UFO hunting), but allows a methodology I believe is the most robust: forced-choice precognition. It avoids many sensory leakage limitations found in other domains, and its odds can be objectively calculated beforehand. The overall aim was simple: rather than potentially add to the pile of inconclusive studies on 
the existence of psi, I wanted to find out which factors would most likely lead to a successful demonstration of psi, and then to test these factors. Therefore, individual differences were examined using a previously successful experimental paradigm, and tested in both a replicatory experiment and in the real world. While the overall results leaves more to be desired, I hope it paves the way for structured research along similar lines. 


\section{TABLE OF CONTENTS}

LIST OF TABLES

LIST OF FIGURES

CHAPTER ONE: WHAT IS PARAPSYCHOLOGY?

1. Introduction

2. Parapsychology As A Field

3. What Is Precognition?

25

4. The Phenomenology of Precognition

CHAPTER TWO: PRECOGNITION EXPERIMENTS 37

$\begin{array}{ll}\text { 1. Introduction } & 37\end{array}$

2. The Importance of Randomisation $\quad 40$

3. Precognition Experimental Paradigms 43

3.1. Random Choice 46

3.2. Meaningful Choice $\quad 47$

3.3. Computer Task 48

3.4. ESP Deck Order 48

3.5. Physical Objects $\quad 49$

4. Individual Differences 49

CHAPTER THREE: THE META-ANALYSIS $\quad 50$

1. Introduction 50

2. Meta-analysis 52

3. Method 54

3.1. Retrieval of Studies $\quad 54$

3.2. Selection Criteria

3.3. Definitions 56

3.3.1. Independent investigator 56

3.3.2. Individual differences $\quad 57$

3.4. Procedural Features $\quad 57$

3.5. Meta-analysis of Correlation Coefficients 58 
4. Results $\quad 60$

4.1. Descriptives 60

4.2. Major Individual Difference Measures 61

4.2.1. Belief in ESP 61

4.2.2. The Big Five 63

4.2.2.1. Extraversion $\quad 63$

4.2.2.2. Neuroticism 65

4.2.2.3. Openness to experience 66

4.2.2.4. Agreeableness 68

4.2.2.5. Conscientiousness 69

4.3.1. Beliefs about luck $\quad 70$

4.3.1.1. Perceived luckiness $\quad 70$

4.3.1.2. Luck belief $\quad 71$

4.3.1.3. Chance belief 72

4.3.1.4. Providence belief $\quad 73$

4.3.1.5. Fortune belief $\quad 74$

4.4. Uncategorised Individual Difference Measures 74

$\begin{array}{ll}\text { 4.4.1. Creativity } & 74\end{array}$

4.4.2. Perceptual defensiveness 75

$\begin{array}{ll}\text { 4.4.3. Transliminality } & 77\end{array}$

$\begin{array}{ll}\text { 4.4.4. Pro attitude } & 77\end{array}$

4.4.5. Dream recall 78

4.4.6. Reports of unusual spontaneous experiences 79

$\begin{array}{ll}\text { 4.4.7. Religiosity } & 79\end{array}$

4.4.8. Emotional reactivity $\quad 80$

4.4.9. Temporal lobe dysfunction 81

4.4.10. Time belief as dynamic 82

4.4.11. Future-orientation 83

4.4.12. Intelligence $\quad 83$

4.5. Single Studies $\quad 84$

5. Summary of Results $\quad 85$

6. Discussion 86 
CHAPTER FOUR: CONFIRMATION 93

1. Introduction 93

1.1. Approach/Avoidance 94

1.2. Affective Priming 95

1.3. Habituation 95

1.4. Facilitation of Recall 96

2. Controversy 97

2.1. Methodological Criticisms 97

2.2. Criticism of Parapsychology 101

3. Responses to the Criticism 103

4. Replication Crisis 105

4.1. Recommendations 108

5. Rationale for Study II 110

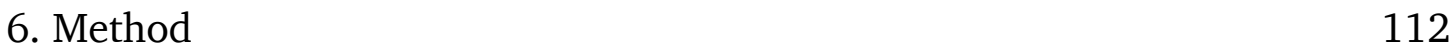

6.1. Participants 112

$\begin{array}{ll}\text { 6.2. Materials } & 113\end{array}$

$\begin{array}{ll}\text { 6.3. Procedure } & 115\end{array}$

$\begin{array}{ll}\text { 7. Results } & 115\end{array}$

7.1. Exploratory Analyses 117

$\begin{array}{ll}\text { 8. Discussion } & 119\end{array}$

CHAPTER FIVE: APPLIED FINDINGS 125

$\begin{array}{ll}\text { 1. Introduction } & 125\end{array}$

2. Rational for Study III(A) 133

3. Method 133

3.1. Participants 133

$\begin{array}{ll}\text { 3.2. Materials } & 134\end{array}$

3.3. Procedure 135

4. Results 135

5. Limitations and Rationale for Study III(B) 144

$\begin{array}{ll}\text { 6. Method } & 147\end{array}$

$\begin{array}{ll}\text { 6.1. Participants } & 147\end{array}$ 
$\begin{array}{ll}\text { 6.2. } \text { Materials } & 147\end{array}$

$\begin{array}{ll}\text { 6.2.1. Extraversion } & 147\end{array}$

6.2.2. Openness to experience 148

6.2.3. Belief in ESP 148

6.2.4. Luck belief 148

6.2.5. Time belief as dynamic 148

$\begin{array}{ll}\text { 6.2.6. Lottery success } & 149\end{array}$

$\begin{array}{ll}\text { 6.3. } \text { Procedure } & 149\end{array}$

$\begin{array}{ll}\text { 7. Results } & 149\end{array}$

8. Discussion 152

$\begin{array}{ll}\text { CHAPTER SIX: CONCLUSION } & 156\end{array}$

1. Introduction 156

2. Overall Findings 158

2.1. The Null Hypothesis 159

2.2. The Psi Hypothesis 163

2.2.1. Sheep-goat effect 164

2.2.2. Precognition 165

2.2.2.1. Quantum theories 166

2.2.2.2. Real-time ESP 166

2.2.2.3. Multiphasic Model of Precognition 167

2.2.2.4. Psi Mediated Instrumental Response Model 168

2.3. Psi As Subjective Probability 169

3. The Non-Significant Results 170

$\begin{array}{ll}\text { 4. Final Thoughts } & 174\end{array}$

$\begin{array}{ll}\text { REFERENCES } & 177\end{array}$

$\begin{array}{ll}\text { APPENDIX A. Study I supplementary tables. } & 209\end{array}$

$\begin{array}{ll}\text { APPENDIX B. Study II materials. } & 210\end{array}$

$\begin{array}{ll}\text { APPENDIX C. Study III(A) materials. } & 228\end{array}$ 
APPENDIX D. Study III(B) materials. 


\section{LIST OF TABLES}

Table $1 \quad$ Summary Statistics of All Individual Difference Measures

Included in Only One Study

Table 2 Summary Statistics of All Individual Difference Measures

That Were Meta-Analysed

Table 3 Descriptive Statistics $(N=204)$ for Belief in ESP,

Extraversion, Openness to Experience, Transliminality,

Magical Ideation, and Psi Performance

Table 4 Comparison of Study II Results with the Confidence Intervals

Found in Study I

Table 5 Matrix of Pearson Correlations Between Predictor Variables and Psi Performance

Table $6 \quad$ Multiple Regression with Belief in ESP, Extraversion, and Openness to Experience Predicting Psi Performance

Table $7 \quad$ Summary and Descriptives of Individual Items on the Precognition Subscale

Table $8 \quad$ Proportion of Sample with Paranormal Beliefs

Table 9 Correlation Matrix Between Lottery Success, Lotto Playing Frequency, Same Numbers Each Week, PBS Score, and Perceived Luckiness

Table $10 \quad$ Multiple Regression Predicting Lottery Success

Table 11 Number of Participants who Submitted Their Predictions

150

Each Week

Table 12 Effect Size Comparisons for Studies I, II, and III

151

Table 13 Multiple Regression Predicting Lottery Success

152

Table A1 Number of Studies for Each Individual Difference Measure 209 that Reported Non-Significance Without Providing Any Other Statistical Information

Table A2 $\quad 68 \%$ and $80 \%$ Confidence Intervals for All Individual 


\section{LIST OF FIGURES}

Figure 1. A flow diagram illustrating the database search and screening stages involved in this meta-analysis.

Figure 2. Meta-analysis of the relationship between belief in ESP and psi performance in forced-choice precognition experiments.

Figure 3. Funnel plot showing the relationship between effect size and sample size (precision) in forced-choice precognition experiments using belief in ESP as a predictor.

Figure 4. Meta-analysis of the relationship between extraversion and psi performance in forced-choice precognition experiments.

Figure 5. Funnel plot showing the relationship between effect size and sample size (precision) in forced-choice precognition experiments using extraversion as a predictor.

Figure 6. Meta-analysis of the relationship between neuroticism and psi performance in forced-choice precognition experiments.

Figure 7. Meta-analysis of the relationship between openness to experience and psi performance in forced-choice precognition experiments.

Figure 8. Funnel plot showing the relationship between effect size and sample size (precision) in forced-choice precognition experiments using openness to experience as a predictor.

Figure 9. Meta-analysis of the relationship between agreeableness and psi performance in forced-choice precognition experiments.

Figure 10. Meta-analysis of the relationship between conscientiousness and psi performance precognition experiments.

Figure 11. Meta-analysis of the relationship between perceived luckiness and psi performance in forced-choice precognition experiments. 
Figure 12. Meta-analysis of the relationship between luck belief and psi performance in forced-choice precognition experiments.

Figure 13. Funnel plot showing the relationship between effect size and sample size (precision) in forced-choice precognition experiments using luck belief as a predictor.

Figure 14. Meta-analysis of the relationship between chance belief and psi performance in forced-choice precognition experiments.

Figure 15. Meta-analysis of the relationship between providence belief and psi performance in forced-choice precognition experiments.

Figure 16. Meta-analysis of the relationship between fortune belief and psi performance in forced-choice precognition experiments.

Figure 17. Meta-analysis of the relationship between creativity and psi performance in forced-choice precognition experiments.

Figure 18. Meta-analysis of the relationship between perceptual defensiveness and psi performance in forced-choice precognition experiments.

Figure 19. Funnel plot showing the relationship between effect size and sample size (precision) in forced-choice precognition experiments using perceptual defensiveness as a predictor.

Figure 20. Meta-analysis of the relationship between transliminality and psi performance in forced-choice precognition experiments.

Figure 21. Meta-analysis of the relationship between pro attitude and psi performance in forced-choice precognition experiments.

Figure 22. Meta-analysis of the relationship between dream recall and psi performance in forced-choice precognition experiments. 
Figure 23. Meta-analysis of the relationship between dream recall and psi performance in forced-choice precognition experiments.

Figure 24. Meta-analysis of the relationship between religiosity and psi performance in forced-choice precognition experiments.

Figure 25. Meta-analysis of the relationship between emotional reactivity and psi performance in forced-choice precognition experiments.

Figure 26. Meta-regression on the relationship between emotional reactivity and psi performance in forced-choice precognition experiments, using publication date as the moderator.

Figure 27. Meta-analysis of the relationship between temporal lobe dysfunction and psi performance in forced-choice precognition experiments.

Figure 28. Meta-analysis of the relationship between time belief as dynamic and psi performance in forced-choice precognition experiments.

Figure 29. Meta-analysis of the relationship between futureorientation and psi performance in forced-choice precognition experiments.

Figure 30. Meta-analysis of the relationship between intelligence and psi performance in forced-choice precognition experiments.

Figure 31. Bar graph showing the distribution of participants' lotto number picks.

Figure 32. Bar graph showing the distribution of participants' 138 correctly predicted lottery numbers compared with MCE (percentage values on top of each column are MCE values).

Figure 33. Pie chart showing the frequency of participants' lottery ticket purchases.

Figure 34. Simple slopes plot for perceived luckiness moderating the relationship between paranormal belief and lottery success. 
Figure 35. Simple slopes plot for lotto playing frequency moderating the relationship between paranormal belief and lottery success.

Figure 36. Simple slopes plot for same lotto numbers moderating the relationship between paranormal belief and lottery success.

Figure 37. Number of participants and their lottery success percentage. 


\section{CHAPTER ONE: WHAT IS PARAPSYCHOLOGY?}

\section{Introduction}

Many people claim to have experienced an event in their lives that seemed to defy scientific explanation (e.g. 29\% of Americans say they have contacted the dead, and 18\% have seen a ghost; Pew Research Center, 2009). These experiences can be described as an anomaly-something that is inconsistent with current understanding of the world. Over the past century, a small and passionate group of psychologists (along with physicists and biologists, amongst others) have studied a very specific type of anomaly known as psi-or anomalous mental phenomena-which is defined as the transfer of energy or information via currently unknown mechanisms (Bem \& Honorton, 1994). In particular, psi refers to two types of ostensibly psychic abilities (Watt \& Wiseman, 2005). The first is extrasensory perception (ESP), which encompasses telepathy (reading others' minds), clairvoyance (perceiving objects not perceivable by ordinary means), and precognition (forecasting the future using paranormal means). The second is psychokinesis (PK), whereby one appears to influence matter without physical contact.

Psi phenomena are typically investigated under the umbrella of parapsychology, which is the scientific study of perceived paranormal phenomena (Irwin \& Watt, 2007). Other anomalous phenomena, such as outof-body experiences and apparitional experiences, are also the subject of study by parapsychologists (see Irwin \& Watt, 2007, for an introduction). By definition, the 'paranormal' is anything that is beyond the reach of scientific understanding (Merriam-Webster, 2018), so 'parapsychology' often invokes ideas of the supernatural by people unfamiliar with the field. Consequently, this type of research is sometimes referred to as anomalistic psychology, a term first coined by University of London Psychology Professor Chris French in an attempt to demystify and create objectivity in the field. Although it might be considered an academic fringe interest, National opinion polls regularly show that the majority of the US population (between 58-67\%; Greeley, 1987) have 
experienced what they believe to be ESP. These anomalous experiences are so widespread that the American Psychological Association has even published a textbook examining the science behind such experiences (Cardeña, Lynn, \& Krippner, 2013)

Irwin (2009) argues that irrespective of whether these phenomena are 'true', there still is merit in studying the human behaviour and experience connected with what is often called the paranormal. He bases this on the fact that a large percentage of the population (over 50\%) endorse at least some form of belief in the paranormal, which means that either (a) paranormal forces really exist, or (b) it tells us something very interesting about human psychology (many people in New Zealand have such beliefs too: see Chapter Five). Irwin (2009) therefore concludes that the field is worth taking seriously, regardless of the resolution of the argument over the existence of psi phenomena.

Although some researchers attempted to examine psi prior to the 1920s, it was only in 1927 that parapsychology emerged as a grudgingly accepted scientific endeavour after Joseph Bank Rhine, a botanist, developed a structured and scientific way to examine psi phenomena. Rhine established America's first parapsychological laboratory at Duke University after being invited to do so by psychologist William McDougall, who had just moved from Harvard to head the psychology department at Duke (Watt, 2016b). While it may seem odd that a plant physiologist was also conducting parapsychological experiments, Rhine was actually in a great position to devise laboratory tests for ESP, as botanists were at the forefront of work in statistical theory in the 1920s (Irwin \& Watt, 2007). His experiments involved Zener cards-a deck of 25 cards each representing one of five simple symbols - as an attempt to avoid the potential biases of traditional playing cards (e.g. people's preference for aces). A typical experiment involved participants guessing the order of a shuffled deck. The number of correct guesses would then be compared and analysed against that which would be expected by chance. Rhine (1934) gathered a huge collection of data from participants tested under controlled conditions (his 167- 
page monograph contains 45 tables, 5 graphs, and extensive documentation), and it was analysed using the most sophisticated statistical tools at the time. Needless to say, it caught a lot of people's attention, as he presented significant results for ESP.

Scientists came in full force to attack Rhine and the statistical methods he used (see Collins \& Pinch, 1979). However, in 1937, the president of the Institute of Mathematical Statistics, Burton Camp, defended Rhine and stated that critics could not fault parapsychological research based merely on statistical grounds (Camp, 1937). Critics therefore turned their attention to methodological weaknesses instead. Today, most researchers accept that Rhine's initial work cannot be taken at face value, as stimulus leakage, or even cheating, can account for his results (J. C. Smith, 2009). For example, any slight indentation on the backs of the cards might have given away extra information, as may the experimenter's speaking tone, facial expressions, or changes in breathing. Such potential challenges have been addressed in a variety of new experimental paradigms that have appeared since then (e.g. Bem, 2011a; Dunne, Nelson, \& Jahn, 1988; Honorton \& Harper, 1974; Soal \& Goldney, 1943; Targ \& Puthoff, 1974; Tart, Puthoff, \& Targ, 1980). It should be noted that these criticisms, and others, have resulted in the adoption of some of the most rigorous of experimental methods in any area of science (Sheldrake, 1999; Watt, 2005).

Overall, however, the evidence does not seem particularly favourable for psi. Although there are promising results published in this area of research (e.g. Bem \& Honorton, 1994; Bierman, 2011; Duane \& Behrendt, 1965; Mossbridge, Tressoldi, \& Utts, 2012; Radin, 2004), these findings have failed to be consistently replicated (Alcock, 2003; Galak, Leboeuf, Nelson, \& Simmons, 2012; Hyman, 2010a). They therefore do not tend to persuade those who were sceptical to begin with (and even some people who are open-minded to the idea). However, every so often a psi experiment with a significant result appears in a high-impact psychology journal, such as the Journal of Personality and Social Psychology or Psychological Bulletin (Bem, 2011a; 
Bem \& Honorton, 1994). But again, it loses mainstream interest rather quickly when other researchers are unable to duplicate the results (see Bierman, 2001; Hansel, 1980; Ritchie, Wiseman, \& French, 2012). Others tend to dismiss the subject matter as not being worthy of any further exploration (Bunge, 1987).

With that being said, some psi research has been more promising than others. In particular, experiments looking at individual differences and psi performance have sometimes found that constructs such as extroversion and/or belief in the paranormal are predictive of psi performance, even when there are no significant findings for psi overall (Honorton, Ferrari, \& Bem, 1998; Lawrence, 1993). These potential psi correlates are important for us to investigate because if psi is real, it will only be useful to us to the extent that we are able to reliably predict, beforehand, the necessary conditions for it to emerge (Hyman, 2010a). Additionally, these correlates might suggest potential mechanisms through which psi might operate. It would not be surprising if some individuals are better at demonstrating psi ability, just as some individuals are more musically gifted. So, in order to move forward with this endeavour, the aim of this research programme is to identify the individual difference measures that have the best potential to predict performance on a psi task (see Chapter Three). These predictors will then be put to the test in a set of varied, yet robust experiments and studies, in an attempt to answer the question of whether psi (and its correlates) exist or not. It is important to examine psi in variety of contexts, as evidence in one setting does not necessarily mean there will be evidence in another (and vice versa).

\section{Parapsychology As A Field}

In general, the scientific community is dismissive of parapsychology, seeing it as something of a taboo subject matter (Mousseau, 2003). In fact, many universities have historically been hesitant to even allow external funding for paranormal research (Wallis, 1979). However, such an outlook neglects that science is a method of enquiry, not a worldview. While the subject matter may seem inconsistent with routine expectations of the stuff of science, scientific 
methodology should be able to objectively dissect the truth or falsity of the subject at hand. If there is zero substance to it, then it should be rather easy to show this.

Nevertheless, some critics continue to label parapsychology as a pseudoscience (e.g. Alcock, 1987; Bunge, 1987; but see French, 2008; Mousseau, 2003, for refutation of this position). That is not to say that there is no justification for using terms, such as pseudoscience, where they are deserved. Anomalistic journals do have far fewer references and a higher proportion of book citations than their mainstream counterparts (Mousseau, 2003), which might be an indication of the subversion of the peer-review process. However, Mousseau (2003) also showed that articles in her sample of anomalistic journals reported more null results than a comparison sample of mainstream journals, so they seem to be less susceptible to publication bias (which many top-tier journals implicitly ignore; see Chapter Four). Furthermore, parapsychology has been instrumental in many methodological developments in psychology, such as the use of blind methods. Fisher (1924) first explored randomisation and statistical inference in card-guessing experiments for the Society for Psychical Research before introducing them to mainstream psychology, and parapsychologists were the first to raise important questions about the efficacy of meta-analysis, after multiple meta-analyses of the Ganzfeld domain (e.g. Honorton, 1985; Hyman, 1985) produced different interpretations of the same data (Rosenthal, 1986). And, as Watt (2005) remarks, "psychology is incomplete if it doesn't include the full range of human experiences, including anomalous experiences," (p. 218) so dismissing anomalies out-of-hand is not the most efficient way to progress our knowledge.

However, Hyman (1995) argues that parapsychology is different to the general study of anomalistics, insofar as the term 'anomaly' is used far more liberally in parapsychology than in any of the other sciences. Specifically, Hyman defines an anomaly as a precise and lawful departure from the norm which needs some explanation to fit into our current framework of understanding, whereas in parapsychology, an anomaly is merely any statistical 
departure from the norm with no defined way to predict when it will occur or how it will present itself (Hyman, 1995). Furthermore, promising anomalies in the other sciences are discarded as soon as they fail to be consistently replicated (e.g. N-rays: Stradling, 1907), which has allowed modern day science to flourish (Hyman, 1964). This does not seem to occur in parapsychology, as its imprecise definition means there is no real way to show that psi does not exist, and therefore the replication attempts go on ad infinitum. Consequently, there seems to be little consensus on what progress has been made in parapsychology, with some sceptics arguing that over the last century, zero progress has been made (e.g. Alcock, 1981) while others have come to the opposite conclusion, in that psi is unequivocally proven and we need to move onto more process-oriented research (e.g. Radin, 2006; Tart, 2002).

There are also other reasons why psychologists, in particular, are more sceptical of parapsychological research than scientists in general (Otis \& Alcock, 1982). Research in cognitive and social psychology have made psychologists much more aware of the biases we tend to exhibit, especially in making intuitive judgments or inferences about things from our day-to-day experience. For example, we know that our memory is unreliable and inaccurate-to the extent that it can be shown to differ from 'reality' (French, 2003). Consequently, psychologists do not give much weight to anecdotal or journalistic claims of the paranormal, since they may be explicable in nonparanormal terms (Bem \& Honorton, 1994). In other words, sceptics argue that the principle of Occam's Razor should apply, whereby the most simple and parsimonious explanation is the one we should accept (i.e. that psi does not exist) and therefore we should not even give parapsychology a second glance. However, a counter principle is Occam's Broom, whereby "inconvenient facts are swept under the carpet in the interests of a clear interpretation of a messy reality" (Vernon, 2015, p. 76). Thus we should be wary of throwing out the baby with the bathwater. Science is always improving on itself and never reaches full closure (Bauer, 2014), so there is nothing to lose by erring in the direction of further research (even if psi does not exist) as it could still bring 
about other related anomalies or discoveries (Utts, 1991).

On the other hand, if psi does exist, then there is much to be lost by not investigating its processes and how these can be enhanced and applied to realworld issues (Utts, 1991). Receiving more support from the scientific community would only help strengthen this resolve (Utts, 1987). Schouten (1993) estimates that the funding for the lifetime of all parapsychological research up until the 1990s is equivalent to just two months of funding for mainstream psychological research in the US for the same period, while Roe (2017) calculates that in the UK, the human resources that have been dedicated to all parapsychological research is equivalent to just 1.2 years of psychology research. This low level of resource allocation towards parapsychology is unfortunate, given that most parapsychologists and sceptics have, perhaps surprisingly, a lot in common (e.g. they both want to eradicate psuedoscientific practices such as alleged 'psychic healing' using sleight of hand techniques, or eliminate the teaching of creationism in a way that prevents critical thinking; Watt, 2005). As Francis Bacon (1909) remarked, "[t] here is a superstition in avoiding superstition" (p. 46), so while we do not want to be too gullible, we also want to avoid being too arrogant and should approach the subject with an open mind.

\section{What Is Precognition?}

As mentioned in section 1, parapsychology encompasses a wide range of anomalous phenomena. However, I will now give an overview of precognition specifically_precognition is the main focus of this thesis given its robustness in the experimental domain (see Chapter Two for the rationale behind this). Starting with its definition, precognition-a type of psi phenomena which also goes by the names of prescience, future vision, or future sight-is the foreknowledge of an event without any known causal mechanism (Honorton \& Ferrari, 1989). It is important to emphasise the word 'known' here because if we were to discover a mechanism that could explain an above-chance prediction of a future event, it would no longer be classified as precognition. 
Rather, the person making the prediction would likely be hailed as an expert in the field in which that the event took place. Indeed, this is the reason why some people get paid to choose stocks or predict what an upcoming trend might be (you're better off saving your money though, as in most cases experts are no better than nonexperts; Tetlock, 2017). The key difference is that 'experts' can use information in the present to inferentially predict what might happen in the future, whereas precognition relies on no such identifiable mechanism; it is often just an alleged feeling that one has information about the future with no awareness of how it came to be. A less loaded term to describe precognition could be 'lucky', but because precognition specifically refers to being 'lucky' when predicting the future, the term precognition is more specific and thus the more appropriate terminology.

In defining what precognition is, it is important to clarify what precognition is not, as there are many instances that seem precognitive but once properly understood, do not constitute precognition. The first and most obvious of these is when someone makes a lucky guess. People have thoughts about the future all the time, so when an event occurs that you had previously thought of, you might intuitively feel like you knew it was going to happen all along (rather than a chance coincidence). This is known as hindsight bias-the tendency for people to think an event was much more predictable than it was, only after-the-fact (Roese \& Vohs, 2012). Obviously, as the popular saying has it-hindsight is $20 / 20$. When you take into account the huge number of times that you have ever thought of a future event, you are going to be right in some of those instances just by pure chance.

However, not all thoughts about the future are the same. Thinking of a common event that does end up occurring (e.g. your friend Sarah will call you) will be much less convincing than an uncommon prediction (e.g. Bob will get hit by a car tomorrow). It is the latter instances that tend to convince a lot of people that precognition is real; in fact, surveys show that $23.5 \%$ of New Zealanders believe in precognition (see Chapter Five). So what can be said of these rare cases? Chance can still account for these instances. A good 
illustration is in the case of dreams that seem precognitive or prophetic. Firstly, there are typically five periods of REM (rapid eye movement) sleep in one night, and because dreams only occur during a period of REM, one might have five periods of dreaming in a single night. Couple that with the 'stories' or 'themes' that occur within a typical REM cycle-upwards of 50-and you then have 250 potential topics that you could potentially dream about in one night (Hines, 2003). If you multiply this by the number of people on the planet, you have up to $1,750,000,000,000$ dream topics per night. A small probability event-such as a dream coincidentally reflecting a future reality-is inevitable (based on the Law of Large Numbers), much in the same way that someone will eventually win the lottery Powerball, even though the odds are 38,383,800 to 1 in New Zealand (NZ Lotteries Commission, n.d.).

Furthermore, while detailed reports of dreams are convincing, the nature of our memory can make us believe that our recollections are more accurate than they really are. One study by Alcock (1981) highlighted this by getting participants to record their dreams as soon as they woke up, to see how accurate they really were; people who came into the experiment reporting dreams that 'always came true' did not find their dreams to be predictive of the future once they wrote them down. It is all too easy to connect an event to a dream you had in the past, which makes it difficult to assess the validity of prophetic dreams or vague precognitive statements in general. For example, if you dreamt that your friend Julie won $\$ 7,000$ in the lottery on Saturday, would a different friend winning $\$ 40,000$ in the lottery on a Wednesday still be considered accurate? If one did not write the specifics down, we would probably consider it a hit.

Another example of what might appear to be precognition is when someone decides to do something on a 'hunch' that something bad is about to occur, and consequently avoids disaster that they would have been caught up in had they not relied on their hunch. There are several points to note about such an event. The first is that we are often not consciously aware of the reasons behind why we do what we do (Pyszczynski, Greenberg, \& Solomon, 2000). So, 
just because we cannot rationalise an action that we have made, does not mean that it was precognitive; you could have been subconsciously acting on minor cues such as the weather outside, the noise of a slightly creaking object, or a person looking suspicious. This is known as implicit learning-where we pick up on subtle non-salient patterns in stimuli without conscious awareness (Reber, 1967). Above-chance predictions may be a consequence of becoming more sensitive to these unconscious or implicit patterns, leading to more accurate predictions (S. Wilson \& Hamlin, 2007).

The phenomenon known as cryptomnesia further illustrates the 'power of our subconscious' (Baker, 1992). Cryptomnesia is the sudden retrieval of past information from memory that one considers erroneously new or original. This has been theorised as a potential root cause in many cases of alleged reincarnation, where vivid details of a past life are described by a 'reincarnee' but are ultimately discovered-sometimes word-for-word—in a book that they read 10 or even 20 years ago, for example (French, 2003).

The power of our subconscious becomes even more complex when you consider a landmark study conducted in the 1980s by Libet and colleagues which reported that unconscious brain activity occurs approximately half a second prior to making a conscious decision (Libet, Gleason, Wright, \& Pearl, 1983). In this experiment, participants were asked to flick their wrist at random moments and record when they made this decision by specifying exactly where the second hand of a clock was. Libet et al. (1983) identified that the electrical brain signal known as bereitschaftspotential—which is a measure of activity in the motor cortex and supplementary motor area of the brain and is related to voluntary muscle movement-always preceded the time on the clock by half a second. Some claim that this is evidence that our decisions are often made on a subconscious rather than conscious level, although this experiment has received its fair share of criticism due its measurement techniques and newer studies showing the possibility of limiting the subsequent action if done within a certain timeframe (Schultze-Kraft et al., 2016). Nevertheless, it is reasonable to argue that our subconscious plays a role in the decisions we make and may 
explain many instances of precognitive 'hunches' that are based on reactions we have to various cues that have not yet entered our conscious mind. While it might sound inconceivable to think that we could be influenced to do things without conscious awareness, we can find plenty of examples in everyday life. For example, millions of people drive to work every day, making hundreds of automatic decisions before arriving at their destinations, then snapping back into reality (Bargh \& Morsella, 2008). Of course, not everyone has the ability to block out familiar stimuli and act on autopilot-individuals that are new to particular tasks, or low in latent inhibition often respond to familiar stimuli as though they were new, and may be overwhelmed by the avalanche of sensory information while driving (Kahneman, 2013). But, in general, we are often guided beyond our current conscious awareness. Other examples include having a quicker reaction time in a word task, based on the ethnicity of a person's name, even though one may not be explicitly aware of this (e.g. Greenwald, Mcghee, \& Schwartz, 1998).

So, if many of these alleged precognitive experiences seem explainable, what then is precognition? It depends on how we use the term. Firstly, if we accept that every event is preceded by a cause-in other words, that we live in a deterministic world - then precognition cannot exist, for precognition requires a non-explanation (Irwin \& Watt, 2007). And there is no logical reason why something cannot be explained-we may not understand its causal mechanisms now (if indeed that is the case) but we should be able to understand it once we acquire more knowledge about the world, at least in principle. So in that sense, what we are referring to when we talk of precognition and its related terms is perceived precognition-something that appears paranormal on the surface (i.e. would violate basic limiting principles of science; Tobacyk, 2004). However, the term precognition is still useful in distinguishing that which can currently be explained and that which we have yet to explain. If we do not yet have any plausible explanation for predicting a future event, then precognition seems to be a useful placeholder to describe the situation. 
However, it might be argued that precognition should not be used, because a correctly predicted future event cannot be caused by anything other than currently known causes. To examine whether this is the case, we might look at it philosophically to determine whether precognition is logically possible, at least in principle (before we can be open to causes we do not understand yet). Precognition is the idea that one can have information, in advance, about the future. However, if you have information about a future event, then there is nothing to stop you from changing the future and invalidating what was predicted. This seems paradoxical. For example, if you knew that your cat Ginger was going to get hit by a car tomorrow, you could put her in a cage, lock her in a room, and sit with her for the entire day to avoid that future event occurring. It could be argued that your knowledge of the future actually prevented it from happening-yet who is to say that that event was ever going to happen? In fact, it could not have been the future that you predicted for the precise reason that it did not occur (whether you believe you influenced that outcome or not). However, Rao (1963) argues that if the foreknowledge of an event is more of a potential 'thing' rather than an actual 'thing', then intervention would not invalidate it, given that it would have occurred had you not intervened. Again, this is practically impossible to prove.

There may be a solution to this philosophical conundrum. Most reported precognitive episodes are vague, not believed, not understood, or out of one's control (L. E. Rhine, 1955), so intervention may not even be a real possibility. One can imagine cases where it would seem impossible to change that future outcome-for example, a meteor that was to strike Honolulu at 5pm on May $23^{\text {rd }} 2022$. However, even this future event may be susceptible to change. You could argue that if you knew this would happen, you could plan an elaborate raid on a major weapons factory, hold leading defence missile engineers and scientists hostage, and force them to come up with a plan to divert or destroy the meteor. While this example is ludicrous, there is nothing stopping this possibility from occurring (in principle). Yet it could be argued that while theoretically possible, no person would actually ever do this (as we are in 
reality restrained by antecedent genetic and environmental factors, along with social, legal, and physical boundaries). In other words, precognition might be possible if the person predicting it does not change the future, even if they theoretically could. The second way precognition might be plausible is if the person making the prediction was unaware or uncertain that what they had was knowledge of a future event (i.e. to them, it would just seem like a random thought or guess). This is effectively an extension of point one, in the sense that while they could theoretically do something about it, in reality-due to their belief of the situation - they would do nothing about it (see Brier, 1974, for a discussion of the philosophical arguments and implications of precognition).

The latter is a more plausible manifestation of precognition (if it were to exist) given that there has never been any reliable demonstration of precognitive abilities. For example, Randi's Million Dollar prize for providing a paranormal presentation remains unclaimed, although to be fair, Randi's specifications essentially require proof to the odds of $1,000,000$ to 1 , which is far beyond what is deemed reasonable in most scientific experiments (Randi, 1964). If someone had the ability to predict something in the future with, say, 95\% accuracy (a reasonable expectation for someone who claimed they could predict the future), we would not need any scientific tests to prove this. It would be blatantly obvious to anyone who met that person (and just about anyone else if they ever stepped foot in a casino). However, it would be far less obvious if someone could predict a future event every now and then at a slightly above-chance level, since that same person would also make many failed predictions too. As Broughton (1991) has said, psi may "look like luck" (p. 193). Given that someone having $100 \%$ certainty of a future event seems paradoxical due to the possibility of intervention, we can actually take it one further step and say precognition must look like luck. And this is what is testable in the laboratory (i.e. whether people are sometimes luckier than one would expect by chance alone). Thus, the study of precognition in the scientific domain has an important role to play; it is not necessarily to scientifically analyse macro-level demonstrations of precognition (because we would not 
need science to 'prove' that) but rather, it is to systematically and statistically evaluate the probability of micro-level precognition (which might seem invisible to the 'naked eye'). In other words, we can use science to differentiate between chance-level and above-chance luckiness when it comes to predicting a future event.

\section{The Phenomenology of Precognition}

Before we take precognition into the laboratory, it is important to understand how individuals experience so-called precognition in the real world (or at least claim to experience it). We do need to acknowledge that how an individual might describe their spontaneous experience (an experience that occurs during day-to-day life, rather than in a scientific laboratory) will not necessarily be accurate-even if they are being completely honest—given what we know of biases in memory and cognition (French, 2003). Believers in the paranormal are more susceptible to false memories than non-believers, and are more likely to think that they saw something paranormal occur when it objectively did not (K. Wilson \& French, 2006, 2014). People also unknowingly add false details to their stories and experiences after having discussed an event with someone who has had a similar experience (K. Wilson \& French, 2014), which makes it difficult to take first-hand accounts at face value. Nevertheless, it is still useful to note the trends and subjective accounts of these experiences, as this is the basis for why experimental precognition research is being carried out in the first place; i.e. in order to extrapolate the findings to everyday life (Irwin \& Watt, 2007). We seek to understand why we sometimes experience getting a phone call from someone we haven't spoken to in ten years, for example, right after 'randomly' thinking about them-even if the answer is not paranormal.

Louisa Rhine (1954) collected thousands of case reports of spontaneous precognitive experiences and categorised them into four types: intuitive impressions, hallucinations, realistic dreams, and unrealistic dreams. "Intuitive experiences" are where one has a 'feeling' that something might happen, but 
are not necessarily be able to justify it in any way, whereas "hallucinations" are a message about the future displayed in some sort of sensory experience (Irwin $\&$ Watt, 2007). For example, an individual might imagine a vivid scene playing out in their head and come to find that this scene occurs sometime in the future. "Realistic dreams" are dreams that depict the future in a very literal, rather than metaphorical, way, whereas an "unrealistic dream" is the opposite and may seem fantastical and disorderly, bearing no resemblance to reality (Irwin \& Watt, 2007). For example, the Pharaoh's symbolic dream in the Old Testament, where he dreamt that seven fat cows came out of a river and seven lean cows ate them, in biblical reality predicted seven years of good harvest followed by seven years of bad harvest (Genesis 41:15-36). Sannwald (1963) analysed Rhine's cases and found that the distribution of these cases, from high to low proportion, was: realistic dreams (60\%), intuitive experience (19\%), unrealistic dreams (15\%), and hallucinations (6\%). Similar ratios are supported by other spontaneous precognition case report databases (Drewes, 2002; S. R. Feather \& Schmicker, 2005; Green, 1960; Houran \& Lange, 1998; Orme, 1974).

We cannot take these proportions at face value, as this sample is not entirely random. People were asked to write in to Rhine if they had any of these experiences, so if one of these experiences had been discussed often on the radio, for example, then many more people may have written in about that specific type of experience, potentially inflating the commonality of that experience in the general population (Irwin \& Watt, 2007). Nevertheless, it seems that dreams completely dominate precognitive spontaneous experienceespecially realistic dreams. Rhine (1954) suggested that predictions about the future might be more present in our dreams than our everyday experience, precisely because thinking we know something about the future is so distasteful that it may only be able to impenetrate our minds when our intellectual defenses are down, e.g. during a dream. Alternatively, it might be that more people are comfortable reporting dreams than any other type of experience, since it can be shrugged off as something they have no control over rather than an expression of their own belief system or mental state (Irwin \& Watt, 2007). 
In terms of the emotional significance of the precognitive dreams that people tend to report, Stowell (1997) identified four different types: (1) non-traumatic experiences, (2) bringing guidance to the dreamer, (3) negative situations that are impossible for the dreamer to act on, and (4) situations in which intervention was possible.

What is rather remarkable about the database though, is that these types of experiences are all seemingly subconscious, suggesting that if precognition were real, it would likely not be a conscious awareness of the future (Irwin \& Watt, 2007). In fact, studies have moved away from looking at precognition as a conscious process and instead look at it as more of a subconscious or unintentional process (e.g. Bem, 2011a; Luke \& Morin, 2009, 2014). However, this shift towards the unconscious might be seen as a last grasp for parapsychologists to keep precognition research alive, given the lack of convincing conscious predictions. A sceptic might instead conclude that the most plausible interpretation for spontaneous precognition experiences is a non-paranormal one. People are often unaware of why they have made a choice, picking up on subtle unconscious patterns in the stimuli around themit therefore does not seem too far-fetched to imagine them using this unconscious information to make somewhat accurate predictions about the future (S. Wilson \& Hamlin, 2007).

Another characteristic that Rhine (1954) noted in her case reports is that the individual never experiences the event as though they were in the future or that their experience represented a future event; instead, it was always experienced in present time. Then again, this might be more to do with limitations in our ways of expressing such concepts, as it would be hard to know what a future event could look like other than that event playing in current time. Two studies have also examined the apparent time delay between when the person had their precognitive episode and when the predicted event actually occurred (Green, 1960; Orme, 1974). They found that in approximately $50 \%$ of cases, the predicted event occurred within 2 days and then tapered off substantially afterwards, with only $20 \%$ of cases exceeding 2 
months. A similar trend was reported by Sondow (1988). Again, this might have more to do with the fact that it is easier to remember something that happened recently rather than a long time ago.

Researchers have also examined potential correlates of the belief in precognition. Believers in psi and precognition tend to self-report greater sense of control over political and social events (Tobacyk, Nagot, \& Miller, 1988) and overestimate their ability to control a situation with random outcomes (Blackmore \& Trościanko, 1985; Brugger, Regard, \& Landis, 1991). Perkins and Allen (2006) also found that people who believe in precognition were significantly more likely to report childhood abuse. One explanation might be that precognition (or believing you have this ability) may help mitigate the helplessness that being abused as a child might make you feel, since you could retain a sense of mastery or control and feel less vulnerable to the situation (Cermak \& Rosenfeld, 1987; Hemmings \& Irwin, 1993; Lawrence, Edwards, Barraclough, Church, \& Hetherington, 1995; Ross \& Joshi, 1992). Given that Rudski (2004) found that believers in precognition also evidenced a greater illusion of control, this explanation seems plausible. While some researchers see these beliefs as irrational or negative (Bunge, 1991; Eckblad \& Chapman, 1983; Sjöberg \& Wåhlberg, 2002; Tobacyk \& Wilkinson, 1991), it is entirely logical even for well-adjusted individuals to alter their reality. Oftentimes, we try and create a buffer for ourselves from the stark reality of life, since there are a lot of things that we have no control over but impact us greatly (Perkins \& Allen, 2006). In fact, people who have less realistic views about themselves are generally happier and less depressed than those who have more accurate views about themselves (Bates \& Stevens, 1989; Martin, Abramson, \& Alloy, 1984; S. E. Taylor \& Brown, 1988), which is reflected by believers in precognition reporting more positive self-esteem (Fitzpatrick \& Shook, 1994). Furthermore, it has been argued that such belief systems are actually essential for a sane existence, since we as humans are sufficiently self-aware that confronting our mortality and the unpredictability of life might generate ongoing anxiety (Becker, 1997; Schumaker, 1990). On the other hand, these explanations tend 
to assume that precognition does not exist and that such beliefs are merely 'created' to counteract our reality, which is not necessarily the case.

These case reports, while not providing much evidential value, are helpful in hinting at the different ways in which psi may present itself. We can then create experiments that try to bring out the experiences described, while also coming up with our own hypotheses (Irwin \& Watt, 2007). After all, people have these experiences as part of their normal lives, and we as psychologists should be actively trying to understand the entire spectrum of the human experience. In the following chapter, we will go over the history of experimental precognition research, and explore the most promising experimental paradigms to move forward with. This will hopefully give us the foundation we need to begin our empirical phase with Study I (Chapter Three), in pursuit of answering the ever-elusive question: does psi truly exist? 


\section{CHAPTER TWO: PRECOGNITION EXPERIMENTS}

The preceding chapter gave us an overview of parapsychology, with a focus on experimental psi research (i.e. where the subject matter is either ESP-that is, precognition, telepathy, and clairvoyance-or PK). I then discussed the philosophical implications of precognition, and ended with the phenomenology of precognitive experiences in the real world.

The following chapter will delve into the laboratory side of precognition, as we move towards conducting our own experimental research in this area.

\section{Introduction}

Although precognition has received experimental attention, it is the one form of psi that most parapsychologists feel most uneasy about, according to survey data (Schmeidler, 1971). This is likely to be the case for two reasons: (1) the notion of precognition is paradoxical, as mentioned in Chapter One, and

(2) the data for precognition itself produces the least convincing results in comparison to other psi phenomena (e.g. Bem \& Honorton, 1994; Storm, Tressoldi, \& di Risio, 2012). In other words, the results found in precognition experiments-regardless of their significance levels—are usually very close to Mean Chance Expectation (MCE) (Irwin \& Watt, 2007). Nevertheless, while there are many reasons to be sceptical, there is also a vast amount of literature spanning almost a century of research that deserves to be examined; especially since the people most in need of 'evidence' (e.g. psychological researchers) are no more familiar with recent parapsychological work than the general public (Bem, 2011a; Bem \& Honorton, 1994). While this thesis specifically examines individual differences in a specific experimental paradigm (i.e. precognition forced-choice), I shall begin with a brief overview of the precognition literature to understand where such experiments sit within the bigger picture.

The earliest experimental investigation of precognition began with J. B. Rhine in 1933, with his accumulated results-showing statistical significancebeing published in 1938 (J. B. Rhine, 1938). Most of the early studies were 
with traditional playing cards or Zener cards, in which participants were asked to guess the order of a to-be-shuffled deck of cards. Once the participant made their prediction, the experimenter shuffled the cards and compared their prediction with the newly formed order of the cards. Nobel Leurate Charles Richet is credited as being the first person to apply probability theory to playing card experiments as a test for psychic ability (J. B. Rhine, 1977; Richet, 1884). Once the limitations of human card shuffling became apparent (Rhine claimed that the experimenter could use their own ESP to bias the shuffle), Duke laboratory staff constructed mechanical shufflers which Rhine incorporated into his laboratory experiments, and he continued to find statistical significance for precognition-although to a lesser degree than before (J. B. Rhine, 1941). This suggests that at least some of the variation in Rhine's earlier results may have been due to biased shuffling. Eventually, automated random event generators (REG) were developed using computers and software, and precognition experiments have taken advantage of these ever since, continuing the trend of significant results (e.g. Bem, 2011a; H. Schmidt, 1969).

Recently, there has been more of a shift toward testing for subsconscious or unintentional precognition. For example, rather than only asking participants to predict a future target, researchers also monitor participants for their physiological responses (e.g. heart rate, galvanic skin response, or electrodermal activity), to see whether an increase or decrease in sweat or arousal may be a better predictor of a future event than their conscious response (e.g. Bierman \& Radin, 1997; Bierman \& Scholte, 2002; Mossbridge et al., 2012). For example, Sartori, Massacessi, Martinelli, and Tressoldi (2004) found that participants had a higher heart rate prior to target pictures than nontarget pictures, even though they did not score better than chance when making their actual conscious guesses. These types of experiments are known as presentiment studies, since they measure physiology rather than cognitive aspects; they are still a test of precognition, however, since they are looking for evidence of awareness a future event. 
Perhaps the most well-known series of precognition studies is Bem's (2011a) collection of nine precognition experiments that incorporated standard social psychological and cognitive experimental paradigms, along with implicit psi. In order to test for precognition, Bem reversed the standard procedure, such that the practiced or learned effect occurred only after the participant had made their current choice. For example, in a standard affective priming experiment, positive or negatively emotional words are displayed, followed by a picture showing a positive or negative scene (e.g. Bargh \& Ferguson, 2000). Participants are then asked to push a button indicating the valence of the picture, with congruent words and pictures usually eliciting faster reaction times. However in Bem's (2011a) Experiments' 3 and 4, while the task was the same, the participants had to indicate the valence of the picture before being shown the primed words. Even so, the reaction times were faster when the picture and words were congruent, suggesting that information about the future word went backwards in time to affect participants' current responses. In Experiment 1, Bem used erotic images as the target, based on the success of previous presentiment studies that have been able to elicit physiological responses for extreme images (violent or erotic) ahead of time (e.g. Radin, 1997). However, rather than measure physiology, he employed a simple guessing task (i.e. pick a side, one is correct and will display an image) and hypothesised that if the target image was erotic-which was determined only after participants made their choice-they would be more likely to guess the correct side. This is exactly what Bem found.

Bem (2011a) employed an experimental paradigm known as forcedchoice, in his Experiments' 1 and 2. It is less common than other experimental paradigms in parapsychology, due to it being rather repetitive and not very motivating. In fact, between 1998 and 2007, only six studies used the forcedchoice experimental paradigm in all of ESP research (see Roe, Henderson, \& Matthews, 2008). While not being ideal for ecological validity—since being forced to make a multiple-choice guess about the future is not necessarily depictive of reality—it does have its benefits. Most notably, it is much easier to 
objectively score these studies (cf. free-choice, which requires additional resources to quantify participants' responses), and is also more intuitive and straightforward to participants (Roe et al., 2008). Examples of automated forced-choice procedures include Schmidt's (1969) four-choice lamp device and Honorton's (1987) ESPerciser, a four-option display on the computer that automatically records the participants' choice and determines the target. Reviews and meta-analyses of forced-choice ESP studies, which include not only precognition but also clairvoyance and telepathy experiments, suggest that overall, participants are able to score significantly above-chance in these tasks (Honorton \& Ferrari, 1989; Pratt, Smith, Rhine, Stuart, \& Greenwood, 1940; Steinkamp, 2005; Steinkamp, Milton, \& Morris, 1998).

\section{The Importance of Randomisation}

While precognition experimentation has its benefits (e.g. it is easier to design a study with no sensory leakage, since the target is only determined after the participant makes their choice and thus eliminates potential cues in the present), it is still limited by its randomisation procedures. This is because precognition experiments rely on the chance baseline, or MCE, to effectively be the 'control group' against which participants' choices are compared with. Therefore, inadequate randomisation will likely lead to a Type I error (if the study is adequately powered), since the experimental targets theoretically become predictable; in other words, results may erroneously support the existence of psi phenomena. Although inadequate randomisation is an issue in other domains such as psychology too, the consequence is usually less severe, as the control group and experimental group may be affected similarly (i.e. contain the same biases) or it may not affect the outcome in any tangible way (e.g. an experimenter might put participant $\mathrm{X}$ in the wrong group due to inadequate randomisation, yet the control and experimental group may still remain objectively balanced for all intents and purposes).

As Hyman (1994) remarks, "[a]dequate randomization procedures are critical for parapsychological research because the evidence for psi is based on a 
low probability value for a departure from a chance baseline" (p. 21). Given that humans are known to be poor at "random" guessing (e.g. Ayton \& Fischer, 2004) — we are far more predictable than we think—our patterns of guessing may correlate with the patterns of an inadequately randomised procedure (Utts, 1991; S. Wilson \& Hamlin, 2007). It is problematic, then, that most random functions in computer software actually fail mathematical tests of randomness (L'Ecuyer, 2001). To generate true randomness, the source must come from an indeterminable physical source such as radioactive decay or diode noise (i.e. it cannot be human-made). For example, Schmidt (1969) used a single quantum process for his randomised lamp procedure, where a participant would pick one of four lamps and then the target lamp would be randomly determined (via a radioactive strongtium-90 source) and light up. It is argued that such a single quantum process is nature's most elementary source of randomness $(\mathrm{H}$. Schmidt, 1969). Unfortunately, it is often not practical to use a physical source of randomness, so most experiments incorporate a Pseudo-Random Number Generator (PRNG) instead.

A PNRG works by randomly selecting a 'seed' number (this is often generated from the state of the computer system, such as the time). Based on that seed number, a predetermined sequence of numbers will then be generated whose properties approximate that of a truly random sequence. However, a good PNRG should not be predictable in any real sense to a human, plus, there are also tests one can conduct to ensure that it passes mathematical tests of randomness. PNRGs are often used to mimic true randomness, for example, with slot machines using a PNRG to determine each outcome on a spin. It is therefore imperative that slot machines appear random as much as reasonably possible, or owners' risk financial and credibility issues (otherwise people would start exploiting the machine to win money or stop using it completely). At present, it requires months of reverse-engineering a PNRG, along with the use of sophisticated technology, to have any real way of taking advantage of a sophisticated slot machine (Koerner, 2017). So, for the purposes of randomisation in a precognition experiment, it need only be random enough 
that participants are unable to predict it at all (without the aid of an external device).

Nevertheless, some researchers argue that it is possible to take advantage of a non-random target sequence (even unconsciously), no matter how slight. For example, Gatlin $(1977,1979)$ claims that the degree of nonrandomness in a target sequence is directly related to above-chance scoring for that sequence. In other words, the hit rate in a psi experiment is equivalent to how synchronised the non-random RNG patterns are with the participants' scoring patterns. Brugger and Taylor (2003) take this one step further, arguing that the only thing that all psi experiments show is the weaknesses of randomisation. Not necessarily due to the method of randomisation either but, rather, just by the mere fact that any finite sequence of numbers can never be truly random and free of bias (although this is as much of a philosophical claim as one of objective fact). Thus, when a participant makes their non-random choice (as participants' choices are always biased), it may correlate in some way with the non-random sequence of numbers, whether that results in psi-hitting (i.e. above-chance scoring where both patterns match) or psi-missing (i.e. below-chance scoring, where the patterns do not match). According to Brugger and Taylor (2003), this can account for all significant results in the field of parapsychology.

Furthermore, Hyman (1995) argues that we cannot justify the existence of psi based on deviations from MCE alone. This is because all other scientific fields began with at least one reliable observation before exploring its intricacies, but there has never been such an observation of psi-its presence can only be observed by indirect measures such as rejecting the null hypothesis (since it is negatively defined). The underlying probability model is only "an idealisation of the empirical situation for which it is being used" and since we know that statistical models fit real-world situations only approximately, departures from the null do not "prove" that psi exists (Hyman, 1995, p. 329). In fact, small effects and slight departures from chance are expected to occur, even in the absence of psi, which is why an anomaly based solely on statistical 
inference is problematic (Hyman, 1995). Utts (1995) disagrees, arguing that in any area involving the natural variability inherent in humans, science progresses by first observing a statistical difference and then attempting to explain it. Ultimately, probability inferences underlie nearly all scientific experiments, so parapsychology ought not to be dismissed on the potential weaknesses of statistics alone. An appropriately randomised precognition experiment that can be reliably replicated (with all alternative explanations ruled out) should be given a fair chance to prove itself in the scientific domain. Even if randomisation procedures cannot be truly random, once we accept that we have zero chance of predicting things such as casino games or any other PNRGs (although if psi exists, however weakly, there would be no such thing as having zero chance at predicting things), then those same randomisation procedures ought to be adequate enough to be used as the baseline in psi experiments.

\section{Precognition Experimental Paradigms}

While all precognition experiments test the accuracy of an individual's future prediction, the actual experimental paradigms vary considerably. The experimental paradigms are: (a) free-choice experiments, whereby participants are asked to predict a future event and can come up with any answer they like-that is, they can make an unrestricted response, (b) forced-choice experiments, where participants must make a decision on a future event based on a limited number of options, and (c) 'real-world' or field studies, where participants must predict a future event-one that is not experimentally manipulated. While field studies are typically only of limited value (since there are no randomised control groups), the performance of the 'experimental' group in a precognition field study is compared to the MCE in lieu of a 'control' group, and thus can be valid regardless of its setting. There are, though, potential confounds in real-world precognition studies, since there is still the risk that not all possible known causes for above-chance results have been eliminated (e.g. using subtle cues in the environment to predict the future). 
Starting with real-world precognition studies, there have been numerous attempts to establish the accuracy of predictions or 'premonitions' (a term used interchangeably with precognition, but resembles more of an emotional awareness or intuition about a future event rather than a vision; Adams, 2014) with 'premonition registries' being established. These websites allow anyone to register their premonition online before the predicted event takes place (prior to the internet, you could actually send your handwritten predictions to physical offices), in order to assess their accuracy. Unsurprisingly to sceptics, the success of these registered predictions are meager, with only one percent of submissions being predictive of a future event (Ashe, 2001). This outcome is similar to the drop we see in the predictive accuracy of dreams, once individuals start recording their dreams in a dream diary (see Chapter One). In order to ensure the experimental conclusions are valid, they must (a) record the prediction before the event takes place, (b) make sure the prediction is sufficiently precise so that success or failure will be clearly distinguishable, and (c) calculate an estimate of their $\boldsymbol{a}$ priori likelihood (Irwin \& Watt, 2007). For example, if someone were to claim that "there will be quite a big earthquake in Japan," you need to make sure exactly where and when it would occur, how big the earthquake would need to be, and how often Japan has earthquakes. According to official records, Japan has over 1,500 earthquakes per year, so it may not be worth even evaluating this claim. In fact, surprisingly few studies have taken into account all of these factors in real-world studies-and those that have, have mostly yielded null results (e.g. Hunter \& Derr, 1978; McClenon, 1982; Reiser, Ludwig, Saxe, \& Wagner, 1979). However, there are some real-world situations that are more controlled, such as lotteries (see Chapter Five) but, in general, laboratory precognition research is preferable as it is more controlled and reliable.

The next common type of experiment is the free-choice precognition experiment (e.g. Steinkamp et al., 1998). An example of this type of experiment is where a participant is asked to draw a picture of a future event. In this case, as they are able to draw anything they like, it would be classified as a free- 
choice experiment (as they are not limited in what they can draw). The difficulty with analysing free-choice experiments is twofold, however: firstly, it can sometimes be difficult to determine whether there is a match between the participant's subjective output and the predicted target event and, secondly, it is often next to impossible to quantify the a priori odds of getting that prediction correct in the first place. Researchers have come up with ways to solve these issues by creating a set number of targets beforehand that the participant could effectively 'hit' with their response, and thus the responses need only be converted to the closest target match for the probabilities to be quantified. Nevertheless, there is still a great deal of subjective interpretation involved, which is avoided altogether in forced-choice precognition experiments.

The most robust experimental paradigm is the forced-choice precognition experiment, as the odds can always be objectively calculated before the experiment begins due to there being only a limited amount of options that participants can choose from. As early as the 1960s, it was suggested that an easy way to test for precognition was to ask participants to guess from a select few targets that were yet to be chosen, followed by a computer programme randomly generating numbers to be converted to a target (Schmeidler, 1964a). Thus, if a participant were asked to choose a symbol from four options (based on what would appear on the computer screen in the next second), thenassuming that an REG had been configured to display one of these four random symbols with equal probability—we could deduce that the MCE is $25 \%$. Employing common statistical methods used in psychology and other domains, we can objectively determine how likely the participant's result is due to natural variability or manipulated variability (or in other words, whether any above- or below-chance guessing is due to a 'true' effect, or whether these same results are due to chance). The primary downside with forced-choice precognition experiments is that they are typically far-removed from what individuals tend to experience in spontaneous cases of apparent precognition; predictions in the real world tend to be more meaningful and practical (e.g. the prediction may be 
for something that may tangibly affect them, rather than some knowledge of a random target image a few milliseconds away).

Nevertheless, forced-choice experiments provide the best method for producing efficient and objective evidence allowing tests of the notion of precognition, so this thesis focuses primarily on this experimental paradigm. This is based on the fact that even if precognition existed only in the form described in spontaneous cases, then at the very least, these individuals would still be able to predict some things at an above-chance level. The forced-choice paradigm is well suited to help make this determination. Furthermore, as mentioned in Chapter One, the most comprehensive way of understanding the occurrence of apparent precognition is to look at psychological factors in conjunction with the experimental evidence. Therefore, this thesis' focal point is on forced-choice precognition experiments that also assess individual differences that may be associated with precognitive performance. Below I elaborate upon the different types of forced-choice precognition experiments that have included individual differences.

\subsection{Random Choice}

The first and most common type of forced-choice precognition experiment is the random choice experiment, where participants are presented with a few neutral options to choose from-these options might be comprised of digits (Haraldsson, 1975), letters (Haraldsson, 1980; Haraldsson, Houtkooper, Schneider, \& Bäckström, 2002; Haraldsson \& Johnson, 1986), ESP symbols based on Zener Cards (Honorton, 1967, 1972; S. Wilson \& Hamlin, 2007), numbers on a slot wheel (Vaughan \& Houck, 1993), or blank boxes (Haraldsson, 1978; Haraldsson \& Johnson, 1979; Johnson \& Haraldsson, 1984). Participants are then asked to predict which option will be randomly selected as a target in the future. This type of experiment is the easiest to implement as the choices and probabilities are straightforward; e.g. mark one of these four boxes and a random target box will be selected in the future. However, it is also the least interesting for participants, as the options tend to 
be meaningless (on purpose, as it avoids participants having preferences towards any of the choices). For example, participants might be given a sheet of paper with 100 lines and asked to put a number between 0-9 on each line (note that even if participants have preferences for certain numbers, this should not affect the outcome since targets are randomly generated for each participant individually). Following this, target numbers for each line are randomly computed and matched with the participants' choices to determine the success rate. A 'hit' is when the participants' choices match the target.

\subsection{Meaningful Choice}

The second most common type of forced-choice precognition experiment is the meaningful choice experiment where participants are presented a few interesting options to choose from-these include emotion-eliciting words (Freeman \& Nielsen, 1964), brand and product names (Thalbourne, 1996; Thalbourne, Beloff, \& Delanoy, 1982), the location of five "golden discs" within a circular field of 12 circles (Schwartz \& De Mattei, 1983), key points from a dream report (Stanford, 1970), or two-word descriptors (Storm, 2002, 2006a, 2008; Storm \& Thalbourne, 1998-1999, 2001; Thalbourne \& Storm, 2014). Depending on the experiment, participants are either asked to predict which option will be randomly selected as a target in the future, or to choose an option based on the current task (e.g. which two-word descriptors are the most relevant to participants' current feelings). For example, Stanford (1970) required participants to listen to an audio recording of a fictional dream recollection. Following this, participants were asked to recall certain details of

the dream by answering a multi-choice questionnaire-however, unbeknownst to them, the answers were actually indeterminable from the audio recording (i.e. there was no right answer). The target answers were then determined using a random number table and matched with the participants' responses, to determine the 'correct' precognitive hit rate. 


\subsection{Computer Task}

A third type of forced-choice precognition experiment is one that uses a computer programme to conduct the experiment. This type of experiment is becoming increasingly popular in the digital age, as it allows more automaticity and therefore, more trials can be completed in less time. It also avoids the potential of many methodological errors, since experimenters can take a handsoff approach and avoid interfering with both participants' choices and target generation. These experiments typically display a selection of images on a computer screen and the participant must choose which image (or target) they think will be randomly selected by the computer afterwards. Images that have been used in such experiments include erotic images (Bem, 2011a; Wagenmakers, Wetzels, Borsboom, van der Maas, \& Kievit, 2012), positive- and negatively-valenced images (Bem, 2011a), fractal patterns (Hitchman, Roe, \& Sherwood, 2012; Luke, Delanoy, \& Sherwood, 2008; Luke \& Morin, 2014; Luke, Roe, \& Davison, 2008; Luke \& Zychowicz, 2014), playing cards (S. Wilson \& Hamlin, 2007), Zener cards (S. Wilson \& Hamlin, 2007), and a graphical representation of a coin-flip (M. D. Smith, Wiseman, Machin, Harris, \& Joiner, 1997). Participants' choices are considered a 'hit' when the target image matches the participants' choice.

\subsection{ESP Deck Order}

Prior to automated computer tasks, ESP deck order experiments using Zener cards were often employed as a way to test forced-choice precognition (Buzby, 1967b; Johnson, 1969; Schmeidler, 1964a, 1964c, 1964d, 1964b). Such experiments use an ESP deck containing 25 cards of the following symbols: circle, cross, wavy lines, square, and star-five of each. Participants are then asked to predict the order in which the cards of one deck would later appear, and this would constitute one 'run'. The order of the cards are then randomly determined, and participants' choices are compared with this target order. 


\subsection{Physical Objects}

The last type of forced-choice precognition experiment is one that uses physical objects. It is the least common method, as the opportunity for sensory leakage is far higher than with any other method. However, it is common in experiments with small children as they immediately receive feedback, which keeps them interested in continuing the task. Target objects used in these experiments include candy (Drucker, Drewes, \& Rubin, 1977; Winkelman, 1981) and marbles (Winkelman, 1981). Participants guess which colour candy or marble will be revealed from a bag or similar device, and afterwards, the experimenter reaches into the bag (using a glove to avoid tactile cues) and selects a random object as the target.

\section{Individual Differences}

Testing for evidence of precognition is only one half of the scientific study of precognition - the other half is the psychology of paranormal belief and performance, or understanding why and what types of people believe or do well in a psi task. While the latter may not tell us anything about the 'truth' or 'falsity' of precognition, it can help us understand either (a) how precognition works, assuming evidence for precognition comes to light, or (b) how people can form such beliefs (or do well in an experimental psi task), even in the face of conflicting evidence that does not support precognition. This thesis aims to combine both areas of study to produce a better understanding of precognition. Namely, I shall look at experimental evidence for precognition in conjunction with individual differences-that is, the range of constructs that an individual may psychologically vary on, whether that be personality, beliefs, intelligence, or aptitude-to see if patterns can be found that may better explain the results of precognition research. Thus, the next chapter will begin with a meta-analysis of all individual differences predictors of psi performance in forced-choice precognition experiments. 


\section{CHAPTER THREE: THE META-ANALYSIS ${ }^{1}$}

\section{Introduction}

Statistician Jessica Utts (1991) has argued that a "promising direction for future process-oriented research [in parapsychology] is to examine the causes of individual differences in psychic functioning" (p. 377). It seems that such an approach is not only reasonable but also necessary, given that the evidence for psi is often inconsistent and elusive (Kennedy, 2001). If psi is to be taken seriously by the scientific community, its nature needs to be observable under pre-specified conditions (Alcock, 2003; Hyman, 2010a). Individual difference factors-such as specific personality traits (e.g. extraversion) or beliefs (e.g. belief in ESP)—have been previously analysed and thus represent a promising avenue in this regard. However, many researchers ignore individual difference factors (potentially missing important sources of between-individual variation in psi performance) or look at a multitude of varied factors that are difficult to sort through. An actual effect may also be masked if an individual difference factor is systematically related to psi performance. For example, participants who score high on a measure of a trait may over-perform while participants who score low in that trait may under-perform, effectively cancelling each other out. Therefore, the aim of Study I of this thesis is to synthesise the relevant research to better understand the factors that may lead to a successful and consistent demonstration of psi in the laboratory. This will be followed by Study II and Study III, which attempt to replicate and extend the findings of the metaanalysis in Study I.

This meta-analysis focuses specifically on forced-choice experiments that have tested for precognition (i.e. the foreknowledge of an event without any known explanation). Forced-choice experiments give participants several options to choose from for their response, whereas free-choice experiments allow participants to make an unrestricted response. As free-choice experiments—such as the Ganzfeld—have received a lot of attention in recent

\footnotetext{
${ }^{1}$ The analyses reported in this chapter have been published (see Zdrenka \& Wilson, 2017).
} 
literature (see Bem \& Honorton, 1994; Milton \& Wiseman, 1999b; Storm, 2006b), this meta-analysis focuses exclusively on forced-choice experiments. It also focuses on precognition rather than telepathy (anomalous communication between people) or clairvoyance (perception without using normal sensory modalities), as precognition experiments are less susceptible to sensory leakage (Steinkamp, 2005). For example, in some telepathy experiments, participants may potentially make decisions based on the sender's or experimenter's facial cues, but this is not possible in precognition experiments in which the target cannot be known (even by the experimenter) until after the participant has already made their choice.

Individual differences will also be examined, for as Humphrey (1945) succinctly put it,

Progress in science is made by the discovery of the relations [between]...the phenomenon under study to other better-known processes. The finding of a consistent relationship between ESP ability and any mental state or trait, temporary or permanent, would be important. (p. 7)

Furthermore, Wilson and Shadish (2006) suggest that parapsychologists should focus on either producing larger effects or specifying conditions under which researchers would accept the null hypothesis, otherwise more of the same is not going to convince anyone outside of the field. To this end, the meta-analysis will identify the most consistent correlates of psi performance in forced-choice precognition experiments, to help produce greater and more replicable effect sizes.

Previously, there have been three meta-analyses conducted on individual differences in psi laboratory research: one looking at extraversion (Honorton et al., 1998) and the other two looking at belief in ESP, or what is known as the sheep-goat effect (Lawrence, 1993; Storm \& Tressoldi, 2017). All three metaanalyses report a relationship with psi performance $(\boldsymbol{r}$ 's $=.09, .03$, and .05 , 
respectively), indicating a small yet robustly significant overall effect size. However, a variety of other individual differences have been examined in other studies and it would be useful to summarise those studies here, and to compare them all with one another. Furthermore, many meta-analyses combine studies from multiple domains, making it difficult to unpack exactly what factors constitute a replicable psi experiment. Thus, the purpose of this meta-analysis is to (a) provide a comprehensive and updated review of all forced-choice precognition experiments that have included individual difference measures, and (b) estimate the overall magnitude of the relationship between each individual difference measure and psi performance, with the overall goal to provide researchers with the necessary information needed to design confirmatory studies.

\section{Meta-analysis}

Meta-analysis refers to a statistical technique that allows multiple studies to be combined, in order to estimate an overall outcome statistic (see Borenstein, Hedges, Higgins, \& Rothstein, 2009, for more detail). This is advantageous particularly in areas of research characterised by relatively small sample sizes—-that may therefore be underpowered —as the final estimate is effectively based on the $N$ of all observations across all included studies. A meta-analysis is preferred over a literature review, due to meta-analysis being "objectively verifiable, using measured concepts, quantitative data, and statistical analysis" (Bullock \& Svyantek, 1985, p. 112). Meta-analysis is also a great way to separate the noise from the actual psi data (assuming it exists). This is because psi operates within a stochastic framework, which means that it is difficult to claim, on the basis of one precognition experiment (even if the hit rate was $100 \%$ ), that chance did not play a role in the outcome. However, multiple studies in a meta-analysis can help locate the 'psi signal' amongst the noise more than any single study ever could (Storm, 2006b). Lastly, metaanalysis is becoming the gold standard for researchers to understand the current findings of an area-not necessarily as the sole basis to determine 
whether an effect is real, but rather, as a recipe for other independent researchers to follow (Hyman, 2010a).

Some parapsychological meta-analyses have focused on only one research paradigm, such as the Ganzfeld (e.g. Hyman, 1985) or biological systems (e.g. S. Schmidt, Schneider, Utts, \& Walach, 2004), but this is the first meta-analysis to combine both an experimental paradigm and individual differences. Forced-choice precognition is the chosen paradigm, as it is the most efficient method available for replication; the experiments are often automated (less potential for interference from both participants and experimenters) and exact probabilities of hits/misses can be objectively calculated. Although freechoice experiments can also be quantified, this requires an additional step, as participant responses need to be converted to target responses. This is avoided in forced-choice experiments altogether.

Although forced-choice precognition experiments might seem too narrow a subset to analyse, Steinkamp (2005) reviewed all forced-choice ESP experiments—including telepathy, clairvoyance, and precognition—and found it difficult to come to any conclusions due to conflicting outcomes and wide variations in study designs. Furthermore, while some studies do not show any differences in effect sizes between precognition and other domains (see Steinkamp et al., 1998), other studies have found a difference between clairvoyance, precognition, and telepathy effect sizes (e.g. Storm et al., 2012; Tart, 1983). Therefore, in defining the inclusion criteria narrowly, we sought to overcome the heterogeneity of studies in Steinkamp's (2005) review.

For the purposes of this meta-analysis, the effect size of interest is the correlation coefficient between the individual difference measure and psi performance - not psi performance specifically—with the participant as the unit of analysis. 


\section{Method}

\subsection{Retrieval of Studies}

Only studies in the published literature are included in the meta-analysis, since parapsychology is a relatively small field and it is unlikely that there are many unpublished dissertations or theses (Honorton \& Ferrari, 1989). Sourcing of relevant studies included the bibliography of two meta-analyses (Honorton \& Ferrari, 1989; Storm et al., 2012), a database search (described below), along with an inspection of all English-language parapsychological journals, namely, the Journal of Parapsychology, Journal of the American Society for Psychical Research, Journal of the Society for Psychical Research, Research in Parapsychology, Australian Journal of Parapsychology, European Journal of Parapsychology (including the Research Letter of the Utrecht University Parapsychology Laboratory), and the Journal of Scientific Exploration.

An exhaustive search was conducted of research databases including PsycINFO, Google Scholar, WorldCat, and LexScien, using the keywords "individual differences," "precognition," "parapsychology," "forced-choice," "retrocausation," "retrocausality," "psi," "ESP," and "extrasensory perception." Most of these searches located studies that were already found in the journals listed above.

The search period was intended to capture all published experimental psi research that included individual difference measures (which therefore spanned from 1945 through 2016).

The search strategy revealed 35 individual difference variables including more common measures such as extraversion and belief in ESP, along with less widely used measures such as temporal lobe dysfunction and latent inhibition. 


\subsection{Selection Criteria}

Studies were included from 1945 until 2016 if they met the following criteria:

1. Forced-choice design

2. Precognition design

3. Included individual difference measure(s)

4. A minimum of two human participants

Studies that did not include relevant information were excluded. For example, the results reported in Wiseman and Greening (2002) could not be included as their precognition and clairvoyance data were combined when reporting individual difference measures (e.g. the sheep-goat effect), and results reported by Steinkamp (1998) could not be used as the number of participants was not reported.

The identification, screening, and eligibility of the studies followed the Preferred Reporting Items for Systematic Reviews and Meta-Analyses (PRISMA) guidelines (Liberati et al., 2009). Figure 1 provides a detailed summary of the database search and screening process. 


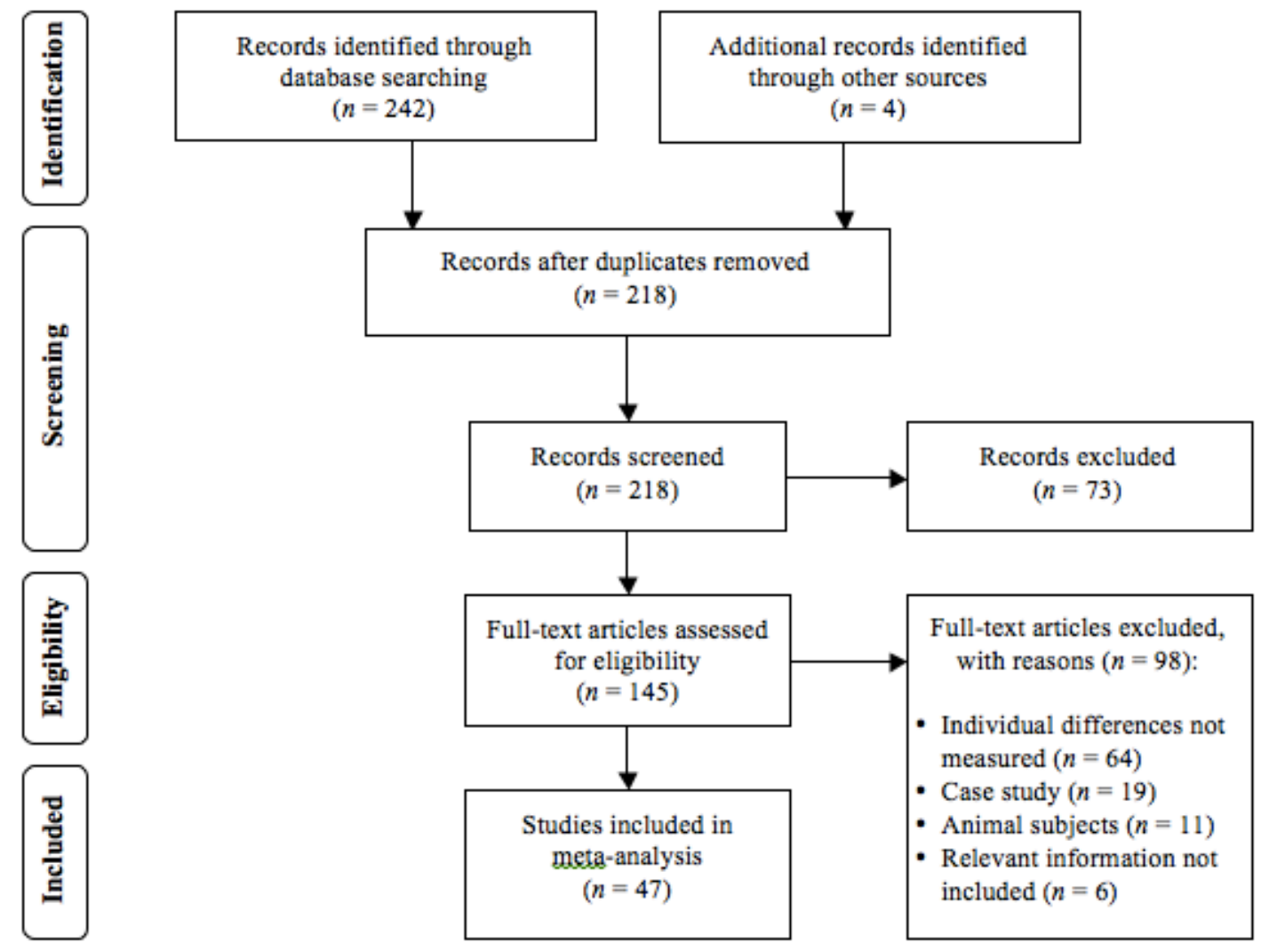

Figure 1. A flow diagram illustrating the database search and screening stages involved in this meta-analysis. ${ }^{2}$

\subsection{Definitions}

3.3.1. Independent investigator. For the purposes of this meta-analysis, an "investigator" refers to the lead author of a study. An investigator is considered independent of another investigator if both investigators have never worked on a paper together using the database being analysed (and/or worked with the other investigator's co-authors). The number of independent investigators can be helpful in determining how replicable an effect might be; the fewer independent investigators there are-even if the studies have been

\footnotetext{
${ }^{2}$ In both the screening stage and the eligibility stage, a number of articles (73 and 64, respectively) were excluded as they did not measure individual differences and were thus not relevant to this meta-analysis. The only difference is that the articles identified for exclusion in the screening stage were deemed irrelevant after only screening the titles, abstracts, and keywords, whereas the others required a more thorough reading of the article to make that determination.
} 
repeated multiple times-the less certain we can be that the results are replicable. As Hyman (1977) notes, it is not enough to simply repeat the same results, for whatever errors or biases may have occurred in the first instance might also be part of the subsequent repetitions of the experiment. Therefore, neutral investigators need to be able to repeat an experimental result also.

3.3.2. Individual differences. For the purposes of this research, an individual difference is defined as anything that an individual may psychologically vary on, whether it is personality, beliefs, intelligence, or aptitude (Nazimuddin, 2015). However, this meta-analysis makes a distinction between individual differences that are relatively constant regardless of situational factors (e.g. trait-level individual differences) and those that are more temporary and can be affected by the experimental situation (e.g. statelevel individual differences, such as participants' mood in the experiment); the latter (e.g. classification of a participant high in trait-anxiety but low in stateanxiety in a particular experimental setting) are not included in this paper to reduce confusion while also limiting the number of variables analysed.

Individual differences were further categorised, combining similar measures (or subcomponents) into families of similar constructs. Where multiple measures of a single individual difference were used in a study, only the most appropriate measure was used. For example, some studies included both a sheep-goat measure and an interest in psi measure-in these cases, only the sheep-goat measure was included, as it is has historically been more consistently used as a measure of ESP belief (Lawrence, 1993).

\subsection{Procedural Features}

Procedural features are those that determine how a study will be analysed and are often included in meta-analysis to help narrow its focus. As there are a multitude of individual difference measures included (35 individual difference constructs were identified for analysis), a separate meta-analysis was performed on each category of individual difference. The majority of these 
meta-analyses contain less than five studies in total, so it was not practical to code for procedural features.

Quality coding of the studies was not implemented for four reasons. Firstly, up until 1976, the founder of experimental parapsychology, J. B. Rhine, encouraged less detail in publications for non-significant parapsychological findings than significant findings (Steinkamp, 2005). Therefore, quality coding would inevitably favour newer studies, since more information is available for post-1976 studies (which may not correlate with the actual quality of the experiment). Secondly, precognition experiments have less potential for procedural defects compared with parapsychological research more generally, which is reflected by Honorton and Ferrari (1989) having only six quality criteria for precognition experiments, in contrast with Pratt, Smith, Rhine, Stuart, and Greenwood's (1940) 34 quality criteria for ESP experiments. Thirdly, Honorton and Ferrari (1989) did not find a relationship between forced-choice precognition experiments and their quality ratings. Lastly, the majority of these meta-analyses had too few studies in total to meaningfully differentiate them on quality.

However, year of publication was coded, as it allowed us to examine whether effect sizes have increased over time, stayed the same, or even decreased. Honorton and Ferrari (1989) suggest that if effect sizes do not increase over time-as they found in their meta-analysis-it might mean that researchers lack an understanding of the underlying factors of psi performance (since they could not reliably increase its magnitude over time). Alternatively, an increase in effect size over time would be more promising, as was reported in Storm et al.'s (2012) meta-analysis.

\subsection{Meta-analysis of Correlation Coefficients}

All indices of association between an individual difference measure and psi performance were converted to correlation coefficients using Comprehensive Meta-Analysis (CMA) software version 2 (Borenstein, Hedges, 
Higgins, \& Rothstein, 2005) or manually. For example, $\boldsymbol{t}$-tests were converted to point-biserial correlations and phi coefficients were computed from $2 \times 2$ contingency tables. Some studies gave only trial-based data such as the critical ratio (z; e.g, Buzby, 1967b; Freeman \& Nielsen, 1964). In these instances, correlations were estimated using a method for calculating effect sizes from critical ratios described by McCarthy and Schechter (1986), providing an estimate of Cohen's $\boldsymbol{d}$ that can then be converted to a correlation coefficient. Unreported correlations were estimated using the provided $\boldsymbol{p}$-values, whereas studies that reported only non-significance had their correlation set to $.00,{ }^{3} \mathrm{a}$ practice consistent with the approach adopted by Honorton et al. (1998) in their meta-analysis of extraversion and ESP performance. Where necessary, correlation signs were adjusted to reflect the appropriate relationship between the individual difference measure and psi performance. Finally, CMA weighted each study-using a random effects model incorporating both sample size and between-study variance-giving an overall outcome metric ( $\boldsymbol{r}$ ) for each individual difference measure in the database. A random effects model was used rather than a fixed effects model, as most studies were not exact replications of each other and this model takes into account such variation (Borenstein, Hedges, Higgins, \& Rothstein, 2010). All $\boldsymbol{p}$ values are two-tailed.

Heterogeneity tests using Cochran's $\boldsymbol{Q}$ were also conducted on each meta-analysis to determine whether results from the included studies were representative of a single homogenous effect. ${ }^{4}$ For those meta-analyses showing heterogeneity, moderator analyses were conducted using the year of publication as a proxy for methodological quality. The $I^{2}$ index was also reported, to give an idea of the degree of heterogeneity present. Finally, Rosenthal's (1979) fail safe $N$, or the file drawer estimate, was calculated for all

\footnotetext{
${ }^{3}$ However, this is an estimate, as the mean of the distribution of all possible non-significant outcomes is likely to be less than zero after removing the outcomes that give significant results by chance (as it effectively removes or truncates the right tail of the distribution). See Appendix A, Table A1, for all studies that this applies to.

${ }^{4}$ Note that these tests were conducted for all meta-analyses, even when there were only two or three studies, as it would be hard to justify an arbitrary cut-off point. However, it does not imply that all of these tests should be given equal weight. The heterogeneity analyses conducted on a limited number of studies should not be considered definitive.
} 
meta-analyses that showed statistical significance to determine how many unreported studies averaging null results would need to exist for the effect to be reduced to overall non-significance. If the number is high, then there is less likelihood for publication bias, that is, studies being reported only if they show statistical significance (Honorton \& Ferrari, 1989). Because unreported nonsignificant studies may have an average effect size below zero (Ferguson \& Heene, 2012), an alternative method for examining publication bias, namely Egger's regression method, was also included for these studies (Egger, Smith, Schneider, \& Minder, 1997). Egger's test aims to quantify potential asymmetrical distributions of studies around the mean effect size (Rothstein, 2008).

Funnel plots were also included for all meta-analyses (with three or more studies) that had significant results, as it gives the reader a graphical depiction of the relationship between effect size and sample size. Large studies tend to appear near the top of the plot (around the mean effect size), whereas smaller studies have greater random variation and will therefore appear more dispersed across the bottom of the plot (Borenstein et al., 2009). Because of the greater random variation in smaller studies, they should be equally likely to appear on either side of the mean; an asymmetrical distribution therefore suggests the existence of publication bias. Given that the visual interpretation of a funnel plot is inherently subjective (Simmonds, 2015), we will also report Duval and Tweedie's (2000) trim and fill analysis, which 'fills in the gaps' and effectively adds the missing studies into the funnel plot that would make it symmetric. In other words, if the test imputes a high number of missing studies, it suggests that some studies having not been reported/published.

\section{Results}

\subsection{Descriptives}

Overall, this meta-analysis is comprised of 57 individual studies, which were reported in 47 papers and conducted by 17 independent investigators. 
The studies span a total of 71 years, between 1945 and 2016. There were a total of 17,634 participants analysed, with sample sizes ranging from 10 to 13,941. In the majority of these studies (49 out of 57), students and/or their associates were the sample population.

Separate meta-analyses of the relationship between psi performance and each category of individual differences are reported below. It is ordered in terms of those variables that have the most exemplar studies (from belief in ESP, the Big Five, various operationalisations of luck, which have the most studies) through to variables for which there are only two or three studies (e.g. religiosity, emotional reactivity, intelligence).

For a summary of the total number of studies, independent investigators, and participants, see Table 2 .

\subsection{Major Individual Difference Measures}

4.2.1. Belief in ESP. Overall, belief in ESP was the most studied potential individual difference correlate in forced-choice precognition experiments, having been reported in 24 studies by 12 independent investigators based on a total of 2,250 participants. The most common measurement questionnaire was a variant of Schmeidler's (1943) sheep-goat criterion, such as Thalbourne and Delin's (1993) Australian Sheep-Goat Scale or Bhadra's (1966) Sheep-Goat Questionnaire. In general, participants who score high on these scales are classified as "sheep" (or believers in ESP) and those who score low are classified as "goats" (or disbelievers in ESP).

Figure 2 shows a forest plot of the correlation coefficients, with the correlations ranging from -.09 to .72. The overall mean weighted effect size $(\boldsymbol{r})$ is .13 ( $p<.001)$, with a 95\% confidence interval between .07 and .20 . This suggests that there is a small but significant relationship between psi belief and performance on a psi task, such that people who believe in psi tend to perform better than those who do not believe in psi. This effect size is slightly larger than the effect sizes reported by both Lawrence (1993) and Storm and Tressoldi 
(2017) in their meta-analyses of the sheep-goat effect ( $\boldsymbol{r}=.03$ and $\boldsymbol{r}=.05$, respectively), but they also included telepathy and clairvoyance experiments.

\begin{tabular}{lrrcc} 
Study name & \multicolumn{3}{c}{ Statistics for each study } \\
\cline { 2 - 5 } & $\begin{array}{rrrr}\text { Lower } \\
\text { Correlation }\end{array}$ & $\begin{array}{c}\text { Cpper } \\
\text { limit }\end{array}$ & Total \\
& 0.04 & -0.19 & 0.26 & 75 \\
Schmeidler, 1945 & 0.30 & 0.17 & 0.42 & 202 \\
Buzby, 1967 & 0.29 & 0.04 & 0.50 & 63 \\
Ryzl, 1968a & 0.31 & -0.09 & 0.63 & 25 \\
Ryzl, 1968b & 0.62 & 0.33 & 0.81 & 28 \\
Johnson, 1969 & 0.72 & 0.28 & 0.91 & 13 \\
Nielsen, 1970 & 0.00 & -0.37 & 0.37 & 28 \\
Honorton, 1972 & 0.00 & -0.13 & 0.13 & 223 \\
Haraldsson, 1975 & 0.11 & 0.02 & 0.20 & 449 \\
Haraldsson, 1980 & 0.00 & -0.20 & 0.20 & 101 \\
Thalbourne et al., 1982 (Exp 1) & 0.30 & 0.09 & 0.48 & 86 \\
Thalbourne et al., 1982 (Exp 2) & -0.06 & -0.25 & 0.14 & 99 \\
Thalbourne, 1996 & 0.06 & -0.22 & 0.33 & 50 \\
Haraldsson et al., 2002 & 0.10 & -0.04 & 0.24 & 199 \\
Storm, 2006 & 0.02 & -0.24 & 0.27 & 61 \\
Wilson \& Hamlin, 2007 (Exp 1) & 0.00 & -0.30 & 0.30 & 44 \\
Wilson \& Hamlin, 2007 (Exp 2) & 0.17 & 0.01 & 0.32 & 149 \\
Storm, 2008 & 0.24 & 0.04 & 0.41 & 100 \\
Luke et al., 2008 & 0.00 & -0.25 & 0.25 & 64 \\
Palmer, 2009 & 0.08 & -0.58 & 0.68 & 10 \\
Luke et al., 2012 & -0.03 & -0.31 & 0.25 & 50 \\
Hitchman et al., 2012 & 0.49 & 0.21 & 0.69 & 41 \\
Luke \& Morin, 2014 & -0.09 & -0.39 & 0.23 & 40 \\
Luke \& Zychowicz, 2014 & 0.01 & -0.27 & 0.28 & 50 \\
Hitchman et al., 2015 (Combined) & 0.13 & 0.07 & 0.20 & 2250 \\
& & & &
\end{tabular}

\section{Correlation and $95 \% \mathrm{CI}$}
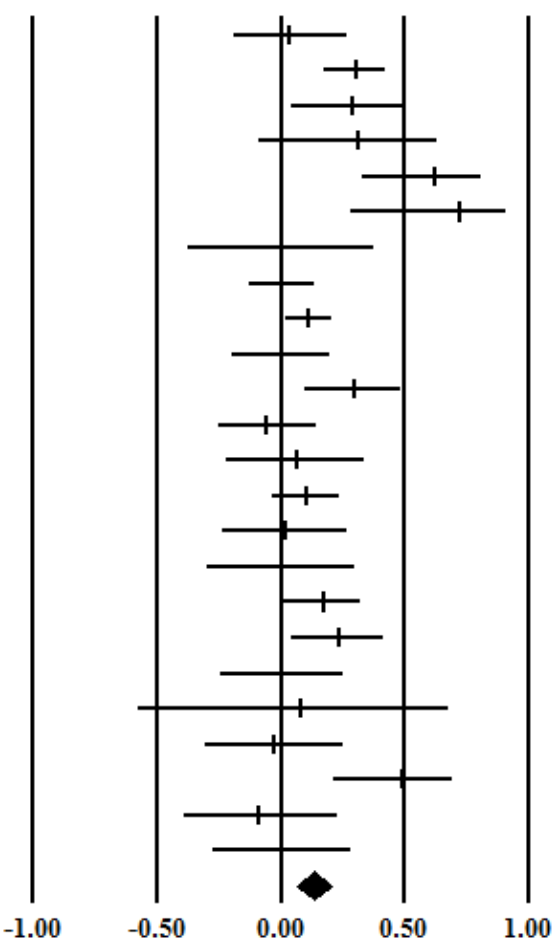

Figure 2. Meta-analysis of the relationship between belief in ESP and psi performance in forced-choice precognition experiments.

However, a test of heterogeneity was significant $(Q=51.07, \boldsymbol{p}=.001)$ which suggests that variation in results may be due to factors other than the relationship between psi belief and performance (for example, error, or the influence of a moderator). The $I^{2}$ was $55 \%$, which also indicates a moderate level of heterogeneity. Consequently, a mixed effects model (method of moments) meta-regression was conducted, but found year of publication not to be a significant moderator, $Q R=2.11, p=.15$.

Finally, the fail safe $\boldsymbol{N}$, or the number of unreported studies averaging null results that would be needed to bring the $\boldsymbol{p}$ value to non-significance, is 
193, and Egger's test was not significant, $t(22)=.88, \boldsymbol{p}=.39$. Using a funnel plot with trim and fill, no additional studies were imputed (see Figure 3 below).

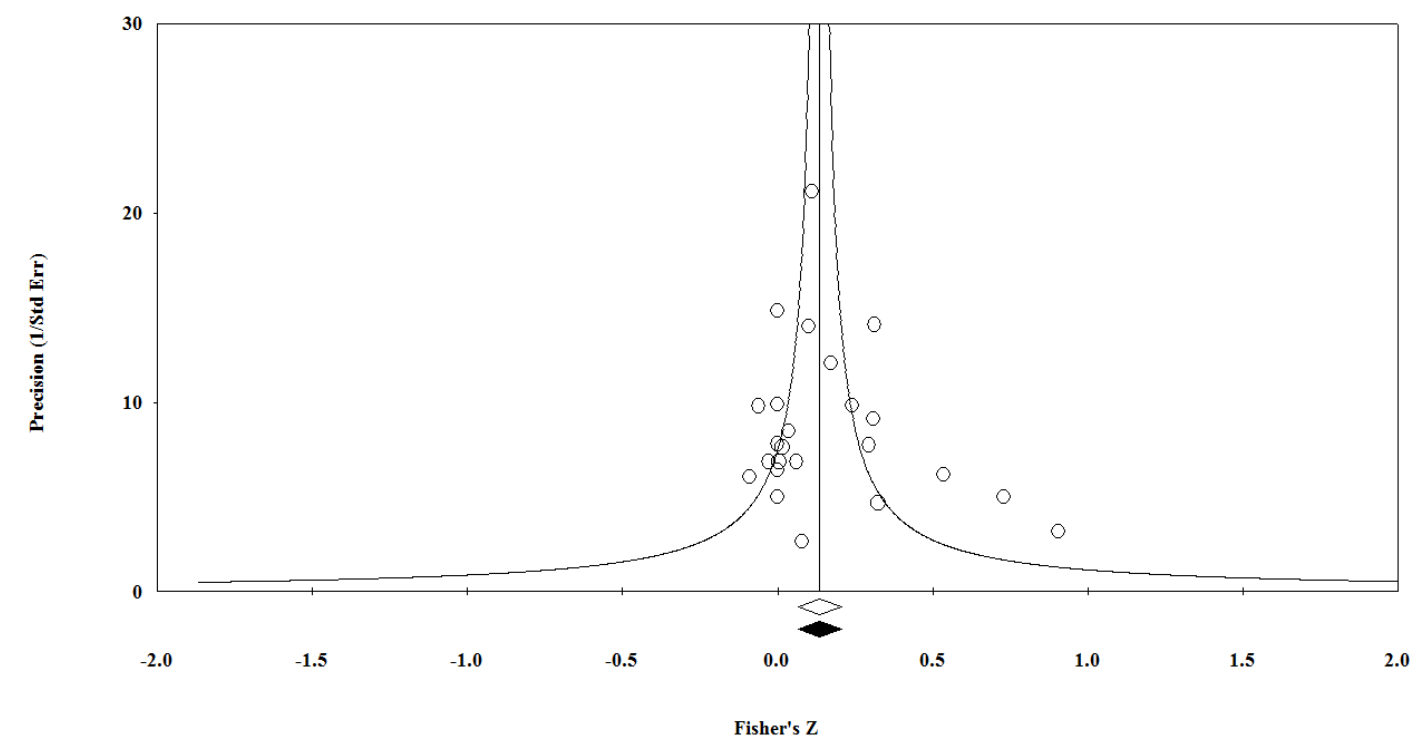

Figure 3. Funnel plot showing the relationship between effect size and sample size (precision) in forced-choice precognition experiments using belief in ESP as a predictor.

\subsubsection{The Big Five}

The next set of meta-analyses cover personality indicators consistent with the Big Five personality traits (McCrae \& Costa, 1987): extraversion (how outgoing and social a person is), neuroticism (a long-term tendency to be in a negative emotional state, such as anxious or frustrated), openness to experience (intellectually curious, willing to try new things, and imaginative), agreeableness (how kind and sympathetic a person is), and conscientiousness (organised and diligent).

4.2.2.1. Extraversion. Extraversion was the second most studied individual difference measure in forced-choice precognition experiments, having been reported in 14 studies by seven independent investigators with a total of 1,206 participants. Extraversion was typically measured within larger 
personality questionnaires such as the 16PF (Cattell \& Mead, 2008), but subcomponents such as Bem's (2011a) Sensation Seeking Scale were also included. High scorers on these measures are generally considered to be extraverted and low scorers introverted.

Figure 4 shows a forest plot of the correlation coefficients, with the correlations ranging from -.28 to .35 . The overall mean weighted effect size $(r)$ is .08 $(\boldsymbol{p}=.02)$, with a $95 \%$ confidence interval between .01 and .15 . This suggests that there is a small but significant relationship between extraversion and psi performance, such that people who are extraverted tend to perform better than those who are more introverted. This result is consistent with previous studies that have also found a positive relationship between extraversion and psi performance (Honorton et al., 1998; Mangan, 1958; Palmer, 1978). Furthermore, a test of heterogeneity was not significant $(Q=$ $17.23, \boldsymbol{p}=.19)$, with an $I^{2}$ index of $25 \%$.

Study name

Humphrey, 1945

Nielsen, 1970

Thalbourne et al., 1982 (Exp I)

Thalbourne et al., 1982 (Exp II)

Storm \& Thalbourne, 1989-99

Storm \& Thalbourne, 2001

Haraldsson et al., 2002

Storm, 2002

Wilson \& Hamlin, 2007

Luke et al., 2008

Bem, 2011 (Exp 1)

Bem, 2011 (Exp 2)

Wagenmakers et al., 2012

Thalbourne \& Storm, 2014

\begin{tabular}{rrrc}
\multicolumn{2}{c}{ Statistics for each study } & \\
\cline { 2 - 3 } Correlation & $\begin{array}{c}\text { Lower } \\
\text { limit }\end{array}$ & $\begin{array}{c}\text { Upper } \\
\text { limit }\end{array}$ & Total \\
0.35 & -0.12 & 0.69 & 19 \\
0.00 & -0.55 & 0.55 & 13 \\
0.00 & -0.20 & 0.20 & 101 \\
0.00 & -0.21 & 0.21 & 86 \\
0.28 & 0.08 & 0.46 & 93 \\
0.00 & -0.19 & 0.19 & 107 \\
-0.03 & -0.31 & 0.25 & 50 \\
-0.03 & -0.33 & 0.27 & 43 \\
-0.28 & -0.53 & 0.02 & 44 \\
0.16 & -0.03 & 0.35 & 100 \\
0.18 & -0.02 & 0.36 & 100 \\
0.17 & 0.01 & 0.32 & 150 \\
0.13 & -0.07 & 0.32 & 100 \\
0.04 & -0.10 & 0.18 & 200 \\
0.08 & 0.01 & 0.15 & 1206
\end{tabular}

Correlation and $95 \% \mathrm{CI}$
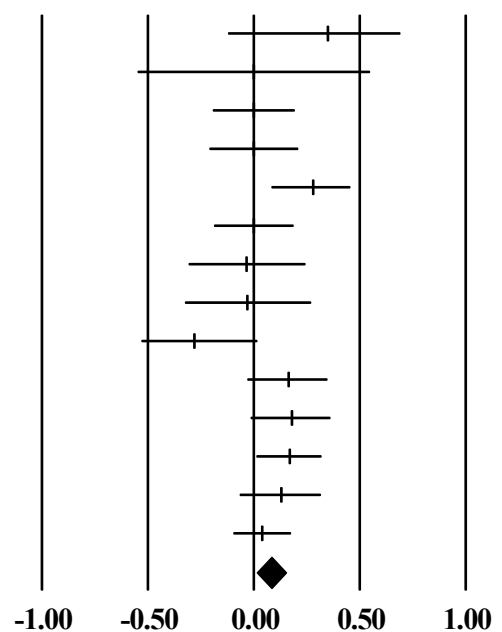

Figure 4. Meta-analysis of the relationship between extraversion and psi performance in forced-choice precognition experiments. 
Finally, the fail safe $N$, or the number of unreported studies averaging null results that would be needed to bring the $\boldsymbol{p}$ value to non-significance, is nine, whereas Egger's test was not significant, $\boldsymbol{t}(12)=0.56, \boldsymbol{p}=.59$. Using a funnel plot with trim and fill, no additional studies were imputed (see Figure 5 below).

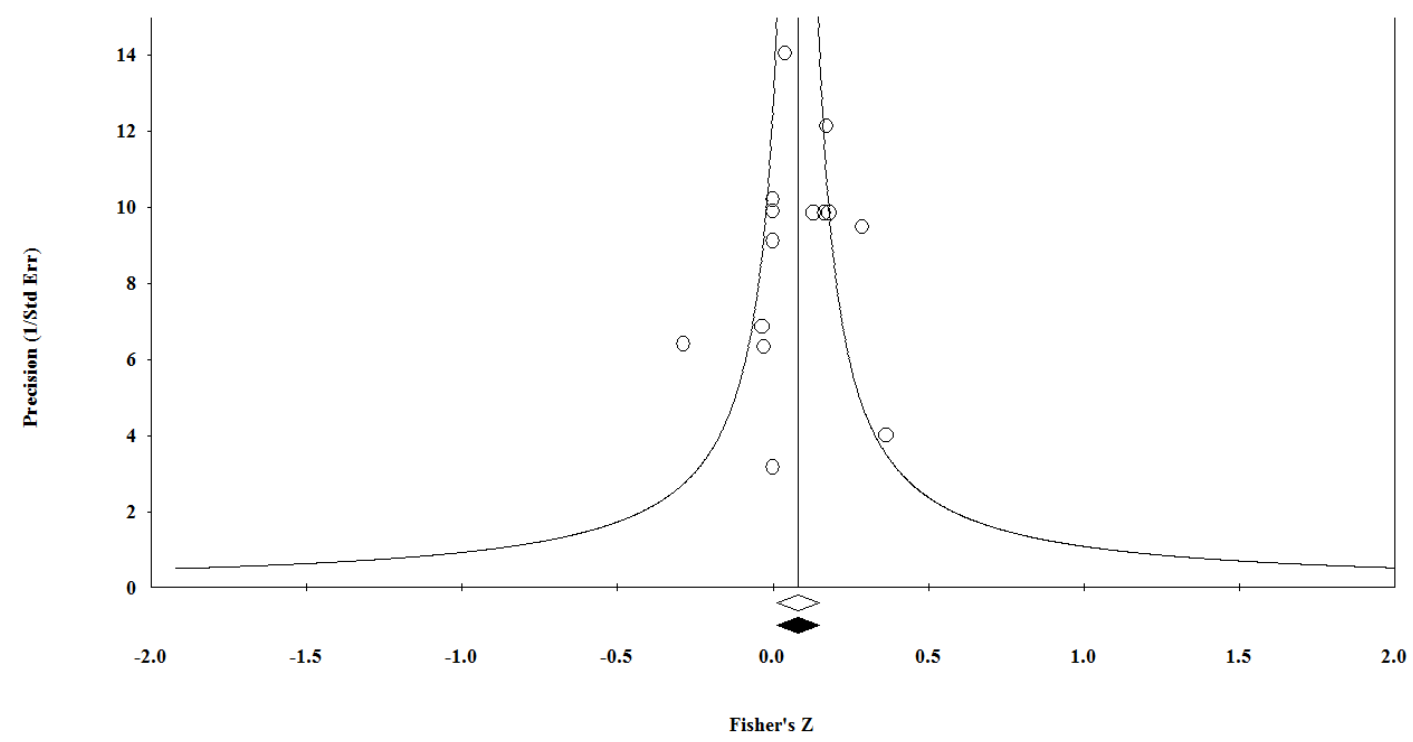

Figure 5. Funnel plot showing the relationship between effect size and sample size (precision) in forced-choice precognition experiments using extraversion as a predictor.

4.2.2.2. Neuroticism. Neuroticism was measured using a variety of different questionnaires encompassing anxiety, affect, and mood, and was included in nine studies by seven independent investigators and a total of 528 study participants. The correlation coefficients range from -.38 to .60 (see Figure 6). The overall mean weighted effect size $(r)$ is .05 $(p=.43)$, with a 95\% confidence interval between -.08 and .19. The results are therefore inconclusive about whether an actual effect occurs, falling slightly short of Steinkamp's (2005) suggestion that neuroticism was a promising predictor of ESP forced-choice experiments.

However, a test of heterogeneity was significant $(\boldsymbol{Q}=17.29, \boldsymbol{p}=.03)$ which suggests that there were potential moderating factors in this database. 
The $I^{2}$ was $54 \%$, which also indicates that there is a moderate level of heterogeneity. As with paranormal belief, a mixed effects meta-regression did not find year of publication to be a significant moderator, $Q R=0.30, p=.58$. Due to meta-regression analyses not being recommended for meta-analyses with less than 10 studies (Borenstein et al., 2009), this finding should be treated with caution.

Study name

Humphrey, 1945

Freeman \& Nielsen, 1964

Thalbourne, 1996

Storm \& Thalbourne, 1998-99

Storm \& Thalbourne, 2001

Haraldsson et al., 2002

Storm, 2002

Wilson \& Hamlin, 2007

Hitchman et al., 2015 (Combined)
Statistics for each study

$\begin{array}{rrcc}\text { Correlation } & \begin{array}{r}\text { Lower } \\ \text { limit }\end{array} & \begin{array}{c}\text { Upper } \\ \text { limit }\end{array} & \text { Total } \\ -0.38 & -0.71 & 0.09 & 19 \\ 0.60 & 0.25 & 0.81 & 23 \\ 0.04 & -0.16 & 0.24 & 99 \\ 0.00 & -0.20 & 0.20 & 93 \\ 0.00 & -0.19 & 0.19 & 107 \\ 0.17 & -0.11 & 0.43 & 50 \\ 0.27 & -0.03 & 0.53 & 43 \\ -0.18 & -0.45 & 0.12 & 44 \\ -0.04 & -0.31 & 0.25 & 50 \\ 0.05 & -0.08 & 0.19 & 528\end{array}$

Correlation and $95 \% \mathrm{CI}$

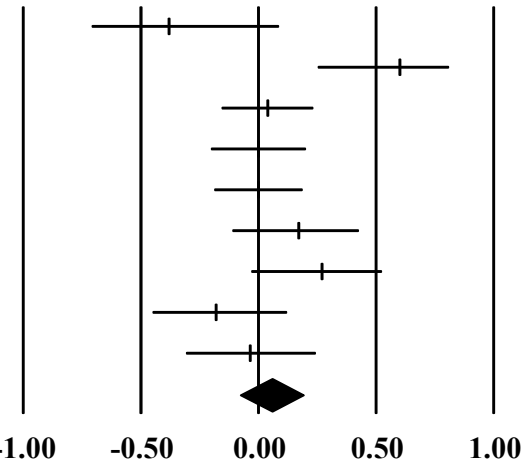

Figure 6. Meta-analysis of the relationship between neuroticism and psi performance in forced-choice precognition experiments.

4.2.2.3. Openness to experience. Openness to experience was reported in 11 studies of 572 participants, by five independent investigators. The most common measurement questionnaire was the Openness to Experience Scale (Goldberg, 1999). Figure 7 shows a forest plot of the correlation coefficients, with the correlations ranging from -.08 to .46 . The overall mean weighted effect size $(r)$ is .12 ( $p=.006)$, with a 95\% confidence interval between .03 and .20 , indicating a small but significant relationship between openness to experience and psi performance, such that people who prefer new experiences tend to perform better than those who prefer routine. Furthermore, a test of heterogeneity was not significant $(\boldsymbol{Q}=11.58, \boldsymbol{p}=.40)$, consistent with an $\boldsymbol{I}^{2}$ index of $5 \%$. 
Storm \& Thalbourne, 1998-99

Correlation $\begin{array}{ccc}\text { Lower } & \text { Upper } \\ \text { limit } & \text { limit } & \text { Total }\end{array}$

Haraldsson et al., 2002

Wilson \& Hamlin, 2007

Luke et al., 2008

Palmer, 2009

Luke et al., 2012

Hitchman et al., 2012

Luke \& Morin, 2014

Luke \& Zychowicz, 2014

Hitchman et al., 2015a

Hitchman et al., 2015b (Combined)

Hitchman et al., 2016

$\begin{array}{rrrr}0.00 & -0.20 & 0.20 & 93 \\ 0.16 & -0.12 & 0.42 & 50 \\ 0.18 & -0.12 & 0.45 & 44 \\ 0.46 & 0.13 & 0.70 & 32 \\ 0.27 & 0.02 & 0.49 & 61 \\ 0.11 & -0.56 & 0.69 & 10 \\ 0.29 & 0.01 & 0.53 & 50 \\ -0.08 & -0.38 & 0.23 & 41 \\ 0.09 & -0.23 & 0.39 & 40 \\ 0.01 & -0.27 & 0.29 & 49 \\ -0.01 & -0.29 & 0.27 & 50 \\ 0.06 & -0.22 & 0.33 & 52 \\ 0.12 & 0.03 & 0.20 & 572\end{array}$

$\mid$

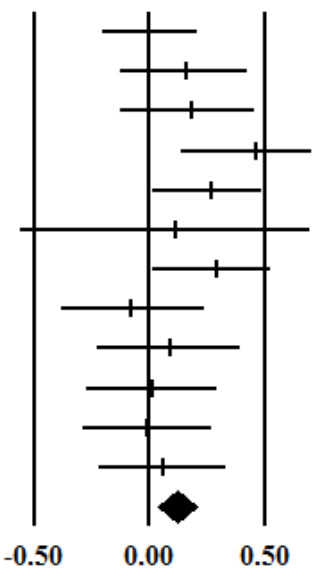

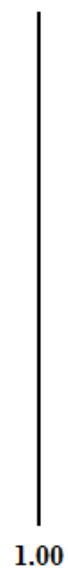

Figure 7. Meta-analysis of the relationship between openness to experience and psi performance in forced-choice precognition experiments.

In this case, the fail safe $N$, or the number of unreported studies averaging null results that would be needed to bring the $\boldsymbol{p}$ value to nonsignificance, is 14. Egger's test was not significant, $\boldsymbol{t}(10)=0.75, \boldsymbol{p}=.47$. Using a funnel plot with trim and fill, no additional studies were imputed (see Figure 8 below). 


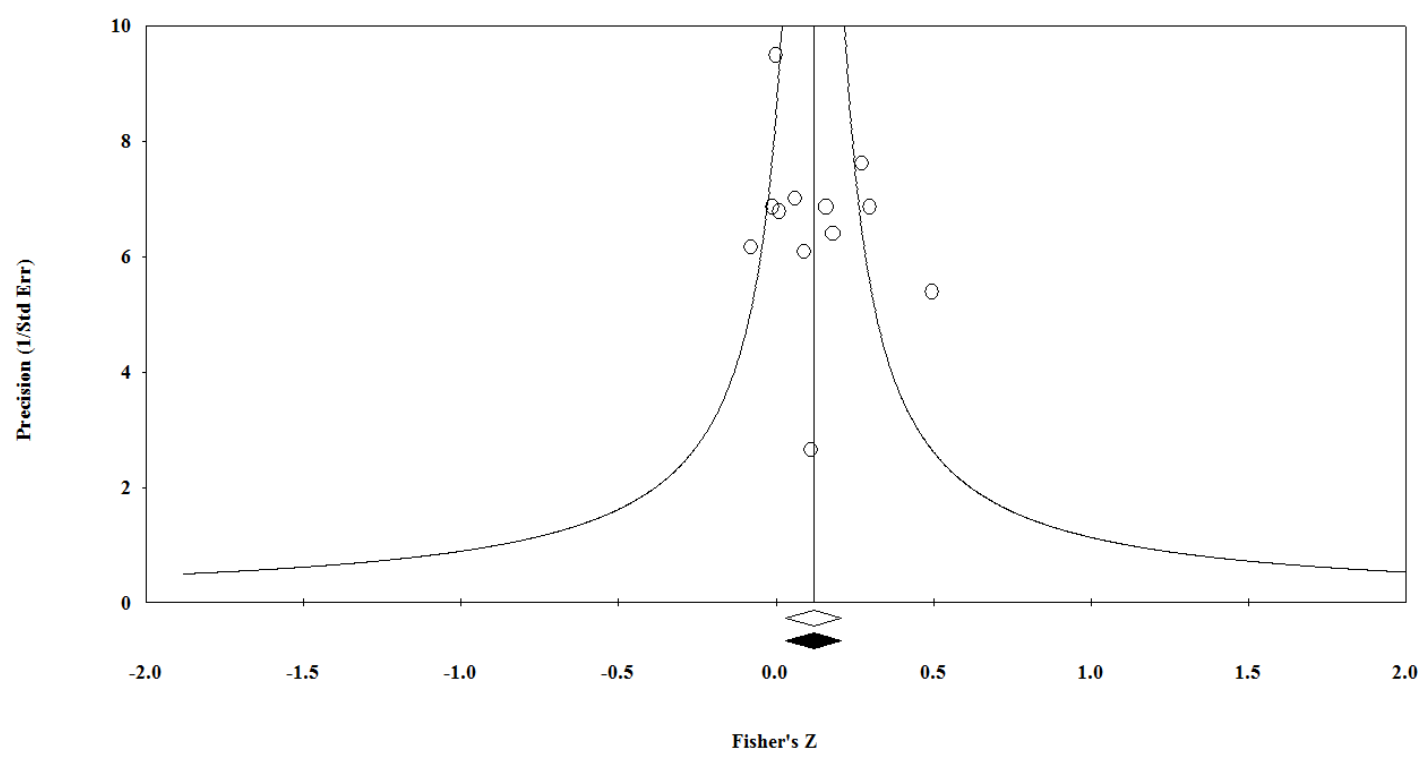

Figure 8. Funnel plot showing the relationship between effect size and sample size (precision) in forced-choice precognition experiments using openness to experience as a predictor.

4.2.2.4. Agreeableness. Agreeableness was reported in seven studies by four independent investigators with a total of 556 participants. The most common measurement questionnaire was the Independence factor of the $16 \mathbf{P F}$ (Cattell, 1996; Cattell \& Mead, 2008). Correlation coefficients range from -.36 to .23 , with an overall mean weighted effect size $(\boldsymbol{r})$ of $.02(\boldsymbol{p}=.71)$ and a 95\% confidence interval between -.09 and .13 (see Figure 9). Although the results are inconclusive, the data suggest that the true effect size is below .13 and could be zero. There was no indication of significant heterogeneity $(Q=$ $9.34, p=.16$ ), with an $I^{2}$ index of $36 \%$. 
Study name

Humphrey, 1945

Storm \& Thalbourne, 1998-99

Storm \& Thalbourne, 2001

Haraldsson et al., 2002

Storm, 2002

Wilson \& Hamlin, 2007

Thalbourne \& Storm, 2014
Statistics for each study

$\begin{array}{rrrr}\text { Correlation } & \begin{array}{c}\text { Lower } \\ \text { limit }\end{array} & \begin{array}{c}\text { Upper } \\ \text { limit }\end{array} & \text { Total } \\ -0.36 & -0.70 & 0.11 & 19 \\ 0.23 & 0.03 & 0.41 & 93 \\ 0.00 & -0.19 & 0.19 & 107 \\ -0.17 & -0.42 & 0.12 & 50 \\ 0.17 & -0.14 & 0.45 & 43 \\ -0.00 & -0.30 & 0.29 & 44 \\ 0.00 & -0.14 & 0.14 & 200 \\ 0.02 & -0.09 & 0.13 & 556\end{array}$

Correlation and $95 \% \mathrm{CI}$

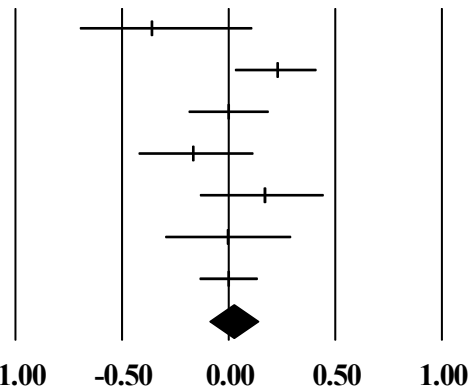

Figure 9. Meta-analysis of the relationship between agreeableness and psi performance in forced-choice precognition experiments.

4.2.2.5. Conscientiousness. Conscientiousness was the least studied Big Five personality trait in forced-choice precognition experiments, having been reported in only three studies by three independent investigators with a total of 187 participants. Conscientiousness was measured as a component of larger personality questionnaires such as the NEO Five-Factor Inventory (McCrae \& Costa, 1987). Correlations range from .00 to .23 (see Figure 10). The overall mean weighted effect size $(\boldsymbol{r})$ is $.06(\boldsymbol{p}=.45)$, with a 95\% confidence interval between -.09 and .20, which is inconclusive but suggests that the effect size may lie below .20 and could be zero. Furthermore, a test of heterogeneity was not significant $(\boldsymbol{Q}=1.69, \boldsymbol{p}=.43)$. The $\boldsymbol{I}^{2}$ is $0 \%$.

Study name

Storm \& Thalbourne, 1998-99

Haraldsson et al., 2002

Wilson \& Hamlin, 2007
Statistics for each study

$\begin{array}{rrrr}\text { Correlation } & \begin{array}{c}\text { Lower } \\ \text { limit }\end{array} & \begin{array}{c}\text { Upper } \\ \text { limit }\end{array} & \text { Total } \\ 0.00 & -0.20 & 0.20 & 93 \\ 0.01 & -0.27 & 0.29 & 50 \\ 0.23 & -0.07 & 0.49 & 44 \\ 0.06 & -0.09 & 0.20 & 187\end{array}$

Correlation and $95 \% \mathrm{CI}$
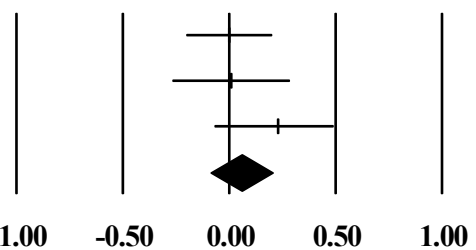

Figure 10. Meta-analysis of the relationship between conscientiousness and psi performance precognition experiments. 


\subsubsection{Beliefs About Luck}

Although luck has been explored in multiple studies, Smith (1998) showed that "luck" can mean different things to different people. Therefore, various measurements of luck and luck beliefs have been reported in forcedchoice precognition experiments that include not only perceived luckiness, but also controllable luck belief, chance belief, providence belief, and fortune belief. The most common tool of measurement used in these experiments was Luke, Delanoy, and Sherwood's (2003) Questionnaire of Beliefs about Luck, which incorporates all of these subcomponents together. They will now be discussed in turn.

4.3.1.1. Perceived luckiness. Perceived luckiness has (prior to 2008) been the standard measurement used to explore luck in psi experiments and refers to how lucky one perceives oneself to be. For forced-choice precognition experiments, perceived luckiness was reported in five studies by two independent investigators with a total of 272 participants. Figure 11 shows a forest plot of the correlation coefficients, ranging from -.20 to .26 with an overall mean weighted effect size $(\boldsymbol{r})$ of $.06(\boldsymbol{p}=.49)$; 95\% CI $[-.11, .23]$. These results are inconclusive but suggest that the effect size is below .28 (and could be zero). A test of heterogeneity was not significant $(\boldsymbol{Q}=7.61, \boldsymbol{p}=.11)$, with an $I^{2}$ index of $47 \%$.

Study name

Smith et al., 1997

Luke et al., 2008a

Luke et al., 2008b

Hitchman et al., 2012

Luke \& Morin, 2014
Statistics for each study

$\begin{array}{rrrr}\text { Correlation } & \begin{array}{c}\text { Lower } \\ \text { limit }\end{array} & \begin{array}{c}\text { Upper } \\ \text { limit }\end{array} & \text { Tota } \\ 0.09 & -0.18 & 0.34 & 56 \\ 0.26 & 0.07 & 0.44 & 100 \\ 0.10 & -0.31 & 0.48 & 25 \\ -0.20 & -0.45 & 0.08 & 50 \\ -0.02 & -0.33 & 0.29 & 41 \\ 0.06 & -0.11 & 0.23 & 272\end{array}$

Correlation and $95 \% \mathrm{CI}$

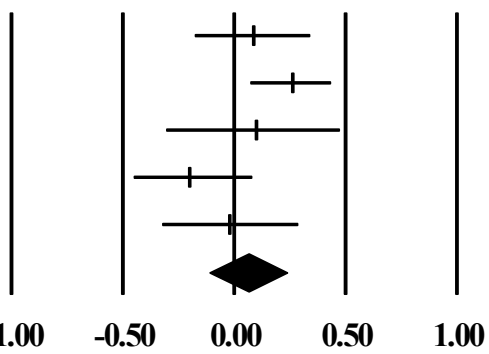

Figure 11. Meta-analysis of the relationship between perceived luckiness and psi performance in forced-choice precognition experiments. 
4.3.1.2. Luck belief. Luck belief refers to the belief that luck is primarily controllable, and participants who score high in this belief also view luck as internal, stable, and non-random (Luke et al., 2003). Luck belief was reported in five studies by one investigator with a total of 248 participants. Figure 12 shows a forest plot of the correlation coefficients, ranging from -.09 to .26. The overall mean weighted effect size $(r)$ is $.13(p=.048)$, with a 95\% confidence interval between .001 and .26, indicating a small but reliable relationship between luck belief and psi performance such that, on the balance of probability, people who believe luck to be controllable tend to perform better than those who see luck as uncontrollable. Furthermore, a test of heterogeneity was not significant $(\boldsymbol{Q}=4.11, \boldsymbol{p}=.39)$. The $\boldsymbol{I}^{2}$ is $3 \%$.

Study name

Luke et al., 2008a Luke et al., 2008b (Study 1) Luke et al., 2008b (Study 2) Hitchman et al., 2012 Luke \& Morin, 2014

\begin{tabular}{rrrr}
\multicolumn{4}{c}{ Statistics for each study } \\
Correlation & $\begin{array}{c}\text { Lower } \\
\text { limit }\end{array}$ & $\begin{array}{c}\text { Upper } \\
\text { limit }\end{array}$ & Total \\
0.26 & 0.07 & 0.44 & 100 \\
0.14 & -0.27 & 0.51 & 25 \\
0.12 & -0.24 & 0.45 & 32 \\
0.04 & -0.24 & 0.31 & 50 \\
-0.09 & -0.39 & 0.22 & 41 \\
0.13 & 0.00 & 0.26 & 248
\end{tabular}

Figure 12. Meta-analysis of the relationship between luck belief and psi performance in forced-choice precognition experiments.

Finally, the fail safe $N$, or the number of unreported studies averaging null results that would be needed to bring the $\boldsymbol{p}$-value to non-significance, is less than 1. However, Egger's test is not significant, $t(3)=1.34, p=.27$. Nevertheless, using a funnel plot with trim and fill, three additional studies were imputed (see Figure 13 below). This increases the imputed point estimate to .24 (with a $95 \%$ CI between .09 and .37), indicating somewhat surprisingly that studies with higher effect sizes may have been unreported. 


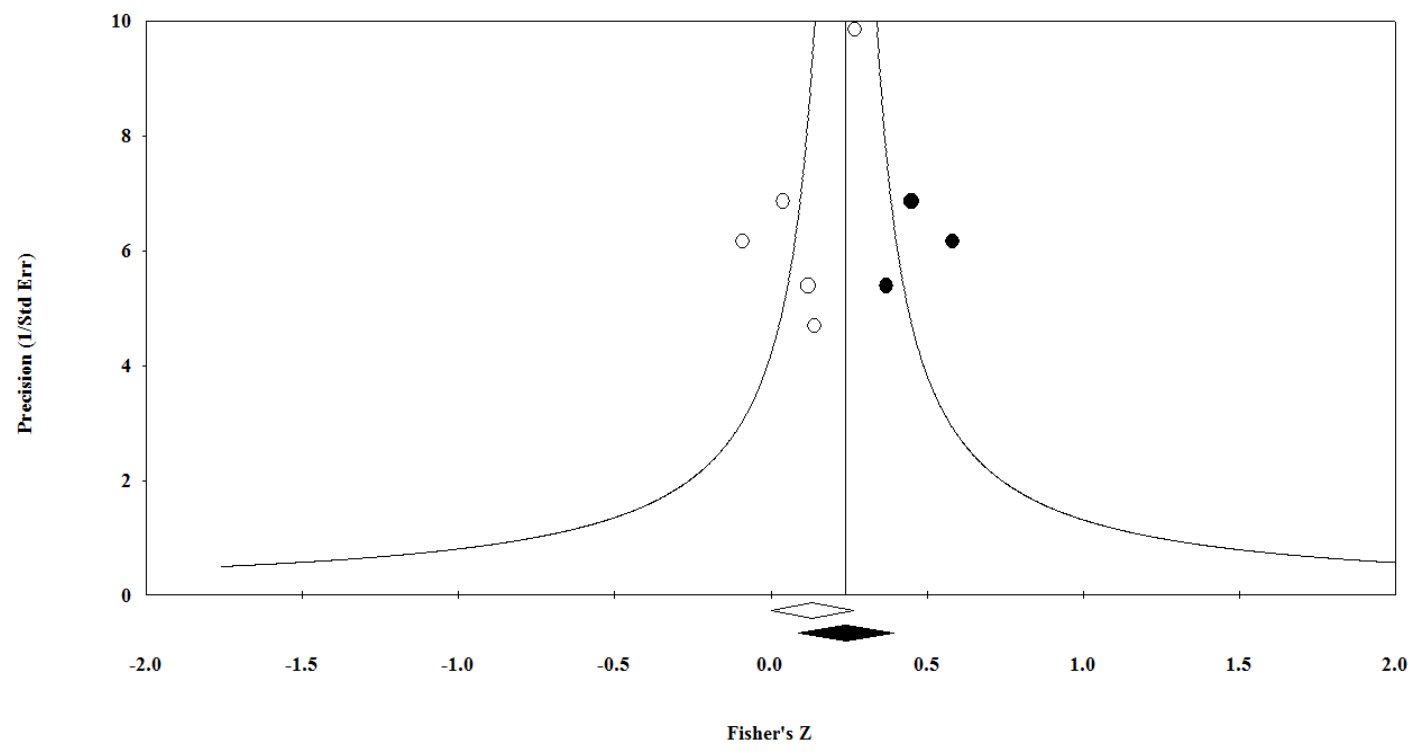

Figure 13. Funnel plot showing the relationship between effect size and sample size (precision) in forced-choice precognition experiments using luck belief as a predictor.

4.3.1.3. Chance belief. Chance belief refers to the belief that luck is random, unpredictable, unstable, and inert (Luke et al., 2003). Chance belief was reported in five studies by one independent investigator with a total of 248 participants. Correlations range from -.16 to .48 (see Figure 14). The overall mean weighted effect size $(\boldsymbol{r})$ is .14 ( $\boldsymbol{p}=.23$ ), with a 95\% confidence interval between -.09 and .36 .

However, a test of heterogeneity was significant $(Q=11.40, \boldsymbol{p}=.02)$ which suggests that there were potential moderating factors in this database. The $I^{2}$ is $65 \%$. A mixed effects model (method of moments) meta-regression was conducted, but it did not find year of publication to be a significant moderator $(Q R=1.75, p=.19)$. 
Study name

Luke et al., 2008a

Luke et al., 2008b (Study 1)

Luke et al., 2008b (Study 2)

Hitchman et al., 2012

Luke \& Morin, 2014 $\underline{\text { Statistics for each study }}$

$\begin{array}{rrrr}\text { Correlation } & \begin{array}{c}\text { Lower } \\ \text { limit }\end{array} & \begin{array}{c}\text { Upper } \\ \text { limit }\end{array} & \text { Total } \\ -0.16 & -0.34 & 0.04 & 100 \\ 0.48 & 0.10 & 0.74 & 25 \\ 0.20 & -0.16 & 0.51 & 32 \\ 0.23 & -0.05 & 0.48 & 50 \\ 0.08 & -0.23 & 0.38 & 41 \\ 0.14 & -0.09 & 0.36 & 248\end{array}$

Correlation and $95 \% \mathrm{CI}$

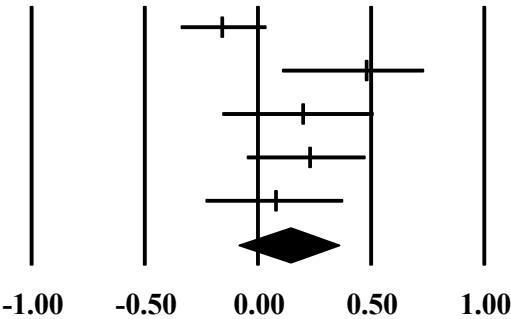

Figure 14. Meta-analysis of the relationship between chance belief and psi performance in forced-choice precognition experiments.

4.3.1.4. Providence belief. Providence belief refers to the belief that luck is something that is managed by external forces or higher beings (Luke et al., 2003). Providence belief was reported in five studies by one investigator with a total of 248 participants. Correlations range from -.09 to .39 (see Figure 15). The overall mean weighted effect size $(\boldsymbol{r})$ is .12 ( $\boldsymbol{p}=.11 ; 95 \%$ CI [-.03, .27]). Furthermore, a test of heterogeneity was not significant $(Q=5.34, \boldsymbol{p}=$ $\left..25 ; I^{2}=25 \%\right)$.

\begin{tabular}{lrrrr} 
Study name & \multicolumn{3}{c}{ Statistics for each study } \\
\cline { 2 - 5 } & $\begin{array}{rrrr}\text { Lower } \\
\text { Correlation }\end{array}$ & $\begin{array}{c}\text { Upper } \\
\text { limit }\end{array}$ & limit & Total \\
Luke et al., 2008a & 0.17 & -0.03 & 0.35 & 100 \\
Luke et al., 2008b (Study 1) & 0.39 & -0.01 & 0.68 & 25 \\
Luke et al., 2008b (Study 2) & -0.03 & -0.37 & 0.32 & 32 \\
Hitchman et al., 2012 & -0.09 & -0.36 & 0.19 & 50 \\
Luke \& Morin, 2014 & 0.23 & -0.08 & 0.50 & 41 \\
& 0.12 & -0.03 & 0.27 & 248
\end{tabular}
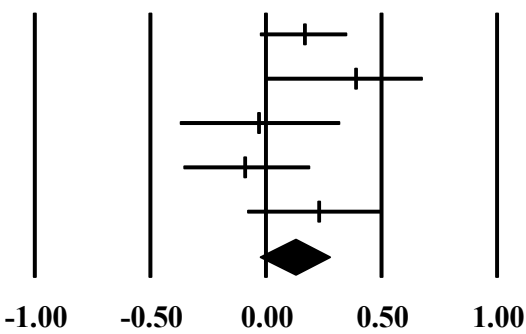

Figure 15. Meta-analysis of the relationship between providence belief and psi performance in forced-choice precognition experiments. 
4.3.1.5. Fortune belief. Fortune belief refers to the belief that luck is meant as a metaphor for life's successes rather than as a literal event (Luke et al., 2003) and was reported in five studies by one independent investigator based on 248 participants. Correlations range from -.08 to .15 (see Figure 16). The overall mean weighted effect size $(\boldsymbol{r})$ is .03 ( $\boldsymbol{p}=.62 ; 95 \%$ CI $[-.10, .16])$. A test of heterogeneity was not significant $\left(\boldsymbol{Q}=1.98, \boldsymbol{p}=.74 ; \boldsymbol{I}^{2}=0 \%\right)$.

\begin{tabular}{lrrrr} 
Study name & \multicolumn{4}{c}{ Statistics for each study } \\
\cline { 2 - 5 } & $\begin{array}{rrrr}\text { Lower } \\
\text { Correlation }\end{array}$ & $\begin{array}{c}\text { Upper } \\
\text { limit } \\
\text { limit }\end{array}$ & Total \\
Luke et al., 2008a & 0.06 & -0.14 & 0.25 & 100 \\
Luke et al., 2008b (Study 1) & 0.15 & -0.26 & 0.51 & 25 \\
Luke et al., 2008b (Study 2) & -0.13 & -0.46 & 0.23 & 32 \\
Hitchman et al., 2012 & 0.12 & -0.16 & 0.39 & 50 \\
Luke \& Morin, 2014 & -0.08 & -0.38 & 0.23 & 41 \\
& 0.03 & -0.10 & 0.16 & 248
\end{tabular}

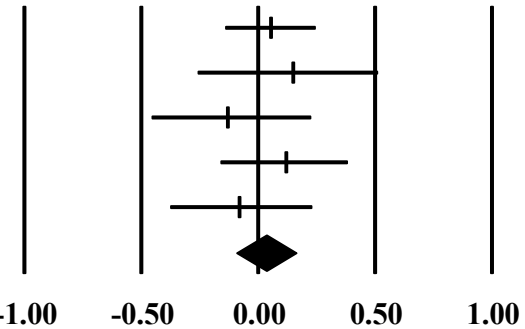

Figure 16. Meta-analysis of the relationship between fortune belief and psi performance in forced-choice precognition experiments.

\subsection{Uncategorised Individual Difference Measures}

4.4.1. Creativity. Creativity was reported in 10 studies by three independent investigators with a total of 546 participants. The most common measurement questionnaires were the short version of the Personal-Social Motivational Inventory (Torrance, 1963) and the Creative Cognition Inventory (Holt, 2002). Figure 17 shows a forest plot of the correlation coefficients, ranging from -.17 to .20 , with an overall mean weighted effect size ( $\boldsymbol{r}$ ) of .06 ( $\boldsymbol{p}=.24 ; 95 \%$ CI $[-.04, .17])$. Furthermore, a test of heterogeneity was not significant $(Q=6.19, p=.29)$, with an $I^{2}$ index of $19 \%$. 
Studyname

Schmeidler, 1945

Honorton, 1967 (Series I, II, III, IV, V conbined)

Luke et al., 2008

Hitchman et al., 2012

Luke \& Zychowicz, 2014

Hitchman et al., 2015 (Conbined)

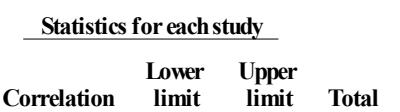

$\begin{array}{llll} & & & \\ -0.17 & -0.38 & 0.06 & 75\end{array}$

$\begin{array}{cccc}0.13 & 0.01 & 0.24 & 300\end{array}$

$\begin{array}{llll}0.20 & -0.16 & 0.51 & 32\end{array}$

$\begin{array}{llll}0.03 & -0.25 & 0.31 & 50\end{array}$

$\begin{array}{llll}0.16 & -0.16 & 0.45 & 40\end{array}$

$\begin{array}{llll}0.03 & -0.26 & 0.30 & 49\end{array}$

$\begin{array}{llll}0.06 & -0.04 & 0.17 & 546\end{array}$
Correlation and $95 \% \mathrm{CI}$

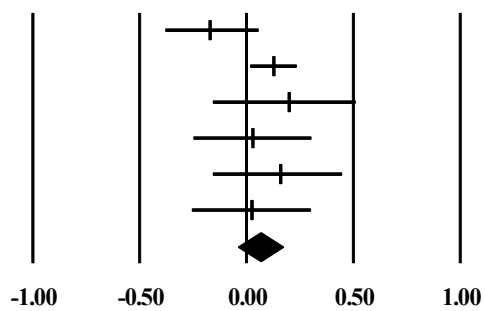

Figure 17. Meta-analysis of the relationship between creativity and psi performance in forced-choice precognition experiments.

4.4.2. Perceptual defensiveness. Perceptual defensiveness refers to psychological defence mechanisms and is argued to be related to subliminal perception and preconscious processing (Haraldsson et al., 2002). All reported studies administered the Defense Mechanism Test (Kragh \& Smith, 1970), with a total of six studies conducted by one investigator and a total of 272 participants. The test incorporates a tachistoscopic technique using peripheral stimuli to trigger subliminal anxiety and thereby defensive reactions. Figure 18 shows a forest plot of the correlation coefficients, with the correlations ranging from -.04 to .30 . The overall mean weighted effect size $(\boldsymbol{r})$ is $.12(\boldsymbol{p}=.049$; 95\% CI [.001, .24]), suggesting a small but significant relationship between perceptual defensiveness and psi performance, such that people who exhibit high preconscious defensiveness tend to perform better than those who do not. There was no evidence of heterogeneity $\left(\boldsymbol{Q}=5.13, \boldsymbol{p}=.40 ; \boldsymbol{I}^{2}=3 \%\right)$. 
Study name

Haraldsson, 1978

Haraldsson \& Johnson, 1979

Johnson \& Haraldsson, 1984 (Study IV)

Johnson \&Haraldsson, 1984 (Study V)

Haraldsson \& Johnson, 1986

Haraldsson et al., 2002

$\begin{array}{rrrr}\text { Correlation } & \begin{array}{c}\text { Lower } \\ \text { limit }\end{array} & \begin{array}{c}\text { Upper } \\ \text { limit }\end{array} & \text { Total } \\ 0.10 & -0.23 & 0.41 & 37 \\ 0.00 & -0.31 & 0.31 & 41 \\ 0.29 & 0.02 & 0.52 & 54 \\ 0.30 & 0.01 & 0.54 & 46 \\ 0.04 & -0.26 & 0.33 & 44 \\ -0.04 & -0.31 & 0.24 & 50 \\ 0.12 & 0.00 & 0.24 & 272\end{array}$

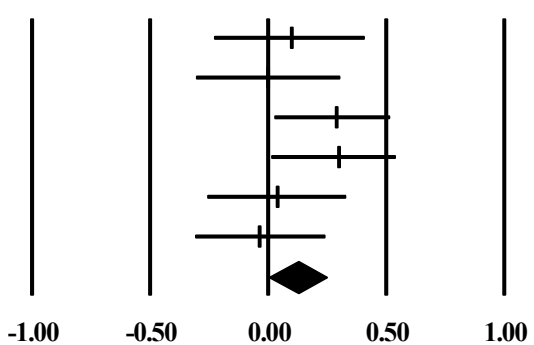

Figure 18. Meta-analysis of the relationship between perceptual defensiveness and psi performance in forced-choice precognition experiments.

In this case, the fail safe $N$ is less than 1 . Nevertheless, Egger's test was not significant, $\boldsymbol{t}(4)=0.79, \boldsymbol{p}=.47$. Using a funnel plot with trim and fill, no additional studies were imputed (see Figure 19 below).

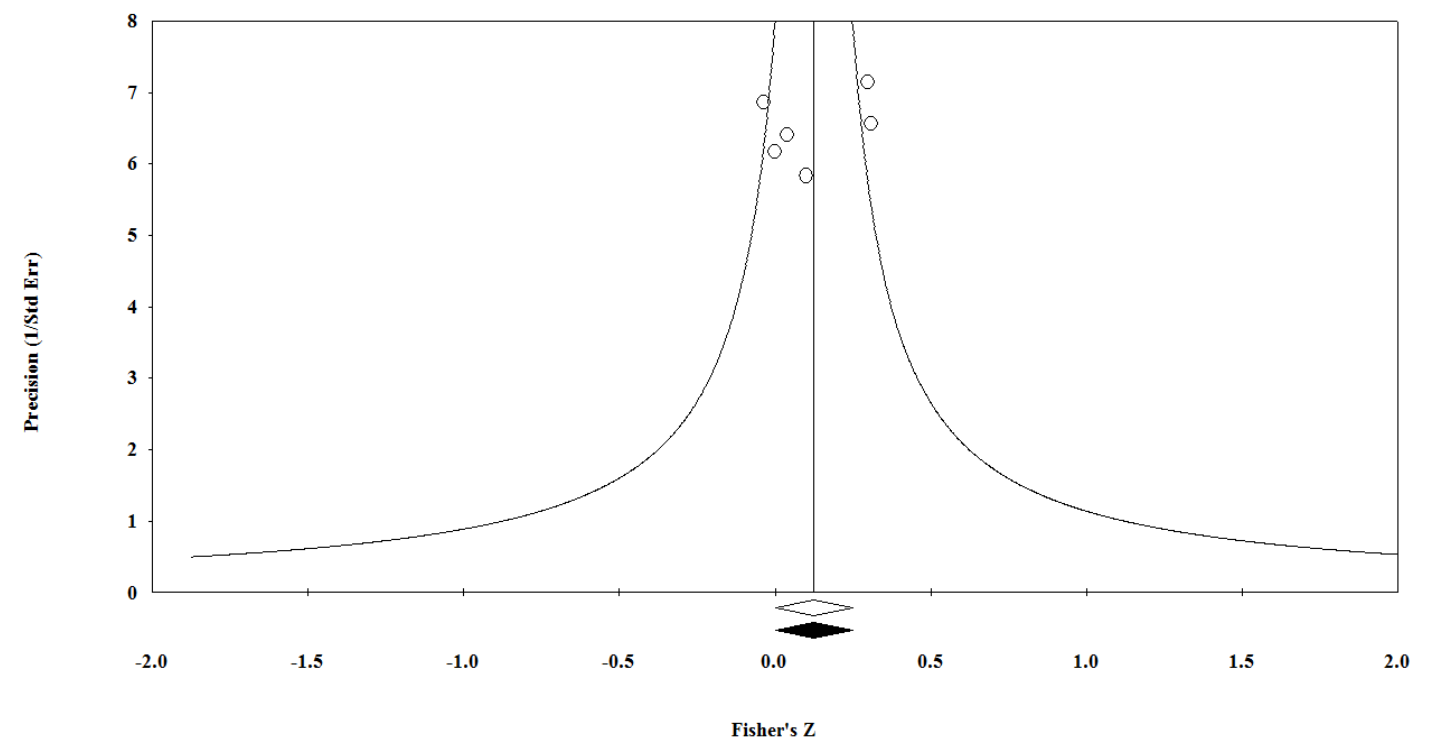

Figure 19. Funnel plot showing the relationship between effect size and sample size (precision) in forced-choice precognition experiments using perceptual defensiveness as a predictor. 
4.4.3. Transliminality. Transliminality is defined as "the hypothesised tendency for psychological material to cross thresholds into or out of consciousness" (Thalbourne \& Delin, 1994, p. 31), and was used in five studies by one investigator based on a total of 542 participants, with the most common measurement questionnaire being the Transliminality Scale (Thalbourne, 1998). Figure 20 shows a forest plot of the correlation coefficients, with the correlations ranging from -.13 to .27. The overall mean weighted effect size $(\boldsymbol{r})$ is .01 ( $p=.91)$, with a 95\% confidence interval between -.13 and .15 .

Although these results are inconclusive, a test of heterogeneity was significant $(\boldsymbol{Q}=9.81, \boldsymbol{p}=.04)$, but year of publication was not a significant moderator $(Q R=1.29, p=.26)$. Due to the small number of studies, this finding should be treated with caution (Borenstein et al., 2009). The $\boldsymbol{I}^{2}$ is $59 \%$.

Study name

Thalbourne, 1996

Storm \& Thalbourne, 1998-99

Storm \& Thalbourne, 2001

Storm, 2002

Thalbourne \& Storm, 2014

\section{Statistics for each study}

\section{Correlation}

Lower

\section{$-0.13$}

0.27

0.01

$-0.01$

$-0.08$

0.01 limit

$-0.32$

0.07

$-0.18$

$-0.31$

$-0.22$

$-0.13$
Upper

limit Total

$0.07 \quad 99$

0.4593

$0.20 \quad 107$

0.2943

0.06200

$0.15 \quad 542$
Correlation and $95 \% \mathrm{CI}$

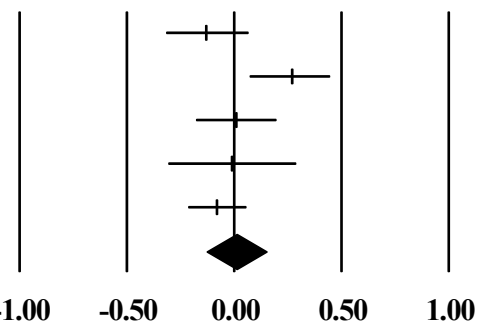

Figure 20. Meta-analysis of the relationship between transliminality and psi performance in forced-choice precognition experiments.

4.4.4. Pro attitude. Pro attitude refers to "an attitude that is favourably directed towards an outcome" (Storm, 2002, p. 47) and three studies with 393 participants were conducted by a single investigator. All used Thalbourne and Storm's (2014) Pro Attitude Scale, which includes items such as "I'm extremely interested in achieving my assigned goal". Figure 21 shows a forest plot of the correlation coefficients, with the correlations ranging from -.16 to .02 , with an overall mean weighted effect size $(\boldsymbol{r})$ of $-.02(\boldsymbol{p}=.70$; CI 95\% $[-.12, .08])$. A test of heterogeneity was not significant $(Q=1.04, \boldsymbol{p}=.60)$, with an $\boldsymbol{I}^{2}$ of $0 \%$. 
$\underline{\text { Study name }} \quad \underline{\text { Statistics for each study }}$

Correlation $\begin{gathered}\text { Lower } \\ \text { limit }\end{gathered} \begin{gathered}\text { Upper } \\ \text { limit }\end{gathered}$

Storm, 2002

$\begin{array}{rrrr}-0.16 & -0.44 & 0.15 & 43 \\ -0.02 & -0.16 & 0.12 & 200 \\ 0.02 & -0.14 & 0.18 & 150 \\ -0.02 & -0.12 & 0.08 & 393\end{array}$
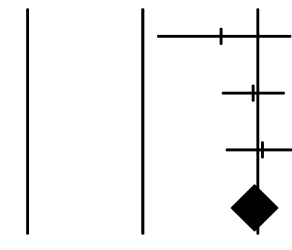

$\begin{array}{lll}-1.00 & -0.50 & 0.00\end{array}$

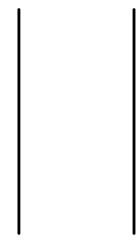

0.501 .00

Figure 21. Meta-analysis of the relationship between pro attitude and psi performance in forced-choice precognition experiments.

4.4.5. Dream recall. Dream recall (specifically, whether an individual recalls their dreams or not) was reported in four studies by three independent investigators with a total of 799 participants. It was typically measured using a one-item questionnaire, which asked participants how frequently they recalled their own dreams. Correlations range from .03 to .43 (see Figure 22). The overall mean weighted effect size $(\boldsymbol{r})$ is .07 ( $\boldsymbol{p}=.23$; 95\% CI $[-.04, .18])$, and a test of heterogeneity was not significant $(Q=5.84, p=.12)$. The $I^{2}$ is $49 \%$.

Study name

$\begin{array}{lrccc} & \text { Correlation } & \begin{array}{c}\text { Lower } \\ \text { limit }\end{array} & \begin{array}{c}\text { Upper } \\ \text { limit }\end{array} & \text { Total } \\ \text { Honorton, 1972 } & 0.43 & 0.07 & 0.69 & 28 \\ \text { Haraldsson, 1975 } & 0.10 & -0.04 & 0.22 & 223 \\ \text { Haraldsson, 1980 } & 0.00 & -0.09 & 0.09 & 449 \\ \text { Thalbourne, 1996 } & 0.03 & -0.17 & 0.23 & 99 \\ & 0.07 & -0.04 & 0.18 & 799\end{array}$

Correlation and $95 \% \mathrm{CI}$

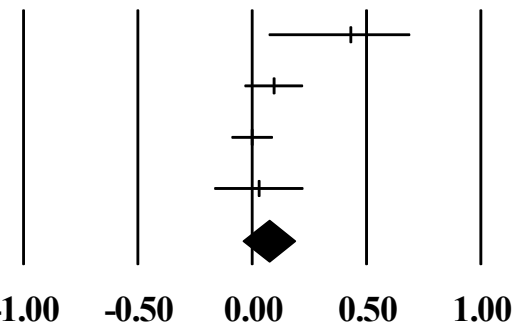

Figure 22. Meta-analysis of the relationship between dream recall and psi performance in forced-choice precognition experiments. 


\subsubsection{Reports of unusual spontaneous experiences. Unusual}

spontaneous experiences can be described as seemingly paranormal experiences in everyday life (as opposed to the experimental laboratory) and were reported in three studies by two independent investigators based on a total of 695 participants. It was typically measured using a single question, which asked participants if they have had any precognitive dreams (i.e. dreams that they thought predicted the future). Figure 23 shows a forest plot of the correlation coefficients, with the correlations ranging from -.21 to .00 and an overall mean weighted effect size $(\boldsymbol{r})$ of $-.01(\boldsymbol{p}=.87 ; 95 \%$ CI $[-.08, .07])$. A test of heterogeneity was not significant $(Q=0.85, \boldsymbol{p}=.65)$, with an $\boldsymbol{I}^{2}$ of $0 \%$.

Study name

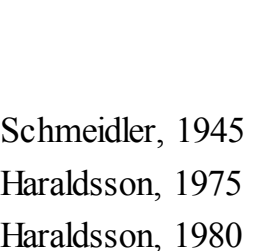

\begin{tabular}{rccc}
\multicolumn{4}{c}{ Statistics for each study } \\
Correlation & $\begin{array}{c}\text { Lower } \\
\text { limit }\end{array}$ & $\begin{array}{c}\text { Upper } \\
\text { limit }\end{array}$ & Total \\
-0.21 & -0.57 & 0.22 & 23 \\
0.00 & -0.13 & 0.13 & 223 \\
0.00 & -0.09 & 0.09 & 449 \\
-0.01 & -0.08 & 0.07 & 695
\end{tabular}

\section{Correlation and $95 \%$ CI}

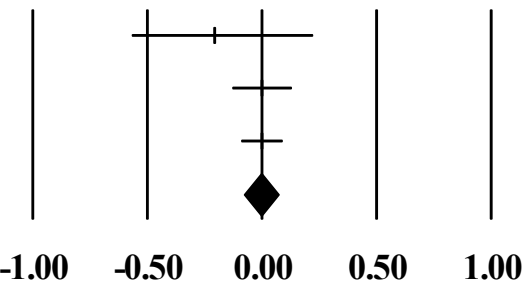

Figure 23. Meta-analysis of the relationship between dream recall and psi performance in forced-choice precognition experiments.

4.4.7. Religiosity. Religiosity was reported in two studies by two independent investigators with a total of 149 participants. In both studies, religiosity was measured using the Religiosity Scale (Haraldsson, 1993), with correlations of -.13 and .08 (see Figure 24). The overall mean weighted effect size $(\boldsymbol{r})$ is $-.05(\boldsymbol{p}=.59 ; 95 \%$ CI [-.24 and .14$])$. A test of heterogeneity was not significant $\left(Q=1.34, p=.25 ; I^{2}=26 \%\right)$. 
Study name

Thalbourne, 1996

Haraldsson et al., 2002
Statistics for each study

Correlation $\begin{array}{ccc}\text { Lower } & \text { Upper } \\ \text { limit } & \text { limit } & \text { Total }\end{array}$

$\begin{array}{cccc}-0.13 & -0.32 & 0.07 & 99 \\ 0.08 & -0.21 & 0.35 & 50 \\ -0.05 & -0.24 & 0.14 & 149\end{array}$

Correlation and $95 \% \mathrm{CI}$

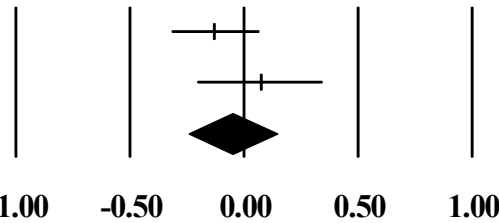

Figure 24. Meta-analysis of the relationship between religiosity and psi performance in forced-choice precognition experiments.

4.4.8. Emotional reactivity. Emotional reactivity is a measure of one's emotional reaction to violent, scary, or gruesome content in photographs, movies, and videos. All studies used the Emotional Reactivity Scale (Bem, 2003). A total of three studies by one investigator looked at emotional reactivity, with 151 participants being included in the experiments. Figure 25 shows correlations ranging from -.27 to .29 , with an overall mean weighted effect size $(\boldsymbol{r})$ of $.06(\boldsymbol{p}=.71 ; 95 \%$ CI $[-.27, .38])$.

\begin{tabular}{lrrrc} 
Study name & \multicolumn{4}{c}{ Statistics for each study } \\
\cline { 2 - 5 } & $\begin{array}{rrrr}\text { Lower } \\
\text { Correlation }\end{array}$ & $\begin{array}{c}\text { Upper } \\
\text { limit } \\
\text { limit }\end{array}$ & Total \\
Hitchman et al., 2015a & -0.27 & -0.51 & 0.01 & 49 \\
Hitchman et al., 2015b (Combined) & 0.17 & -0.12 & 0.42 & 50 \\
Hitchman et al., 2016 & 0.29 & 0.02 & 0.52 & 52 \\
& 0.06 & -0.27 & 0.38 & 151
\end{tabular}

Correlation and $95 \% \mathrm{CI}$

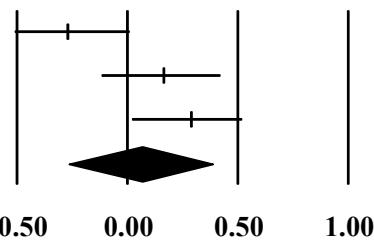

Figure 25. Meta-analysis of the relationship between emotional reactivity and psi performance in forced-choice precognition experiments.

A test of heterogeneity was significant $(Q=8.53, \boldsymbol{p}=.01)$, with the year of publication being a significant moderator $(Q R=3.96, p=.046)$. Figure 26 shows effect sizes to increase as year of publication increases. The $I^{2}$ is $77 \%$. 


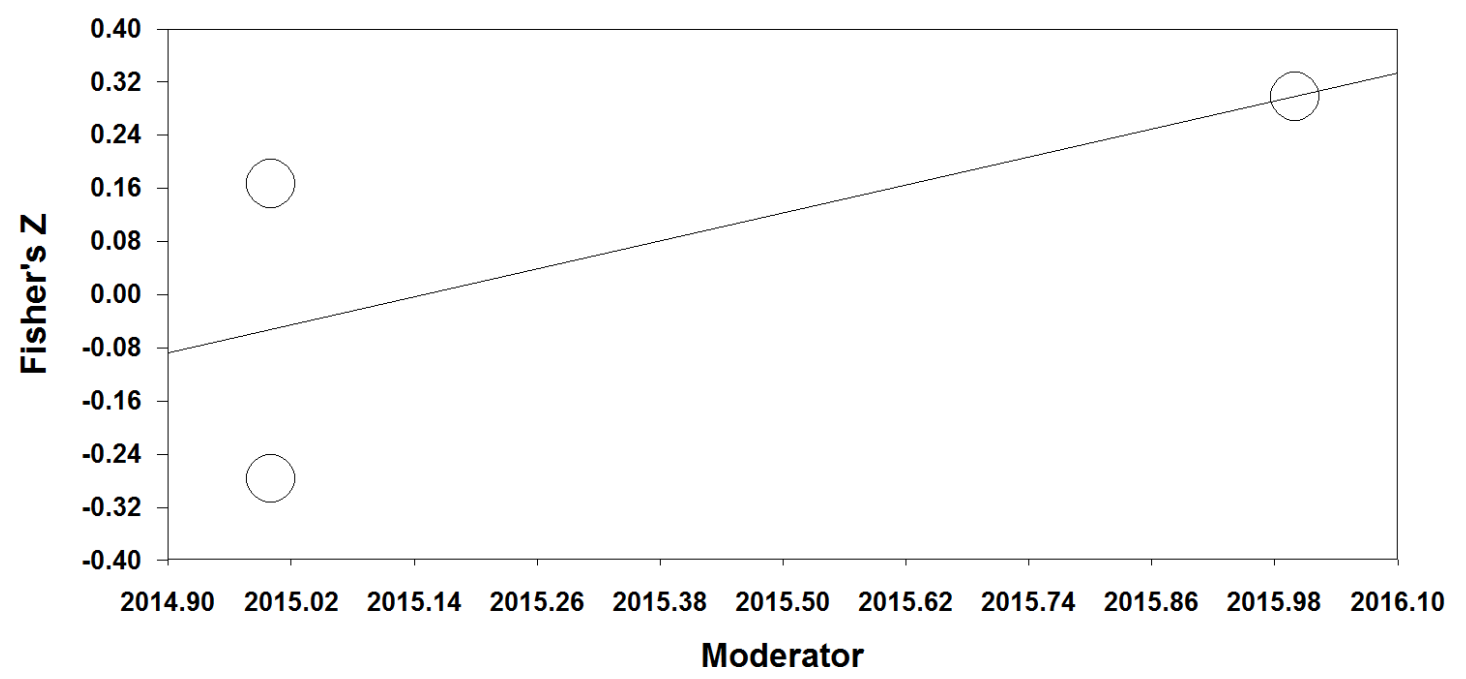

Figure 26. Meta-regression on the relationship between emotional reactivity and psi performance in forced-choice precognition experiments, using publication date as the moderator.

4.4.9. Temporal lobe dysfunction. Temporal lobe dysfunction measures symptoms of temporal lobe damage such as disturbances of perception, selective attention of auditory input, and impaired organisation of verbal material. Two studies by two independent investigators looked at temporal lobe dysfunction, using either the 13-item LIMBEX Scale or the Complex Partial Epileptic Signs cluster of the Personal Philosophy Inventory (Persinger \& Makarec, 1987). There were a total of 114 participants across all of the experiments. Figure 27 shows correlations of -.01 and .00 , with an overall mean weighted effect size $(\boldsymbol{r})$ of $-.004(\boldsymbol{p}=.96 ; 95 \%$ CI $[-.19, .18])$. A test of heterogeneity was not significant $\left.(Q=0.003, p=.96) ; I^{2}=0 \%\right)$. 


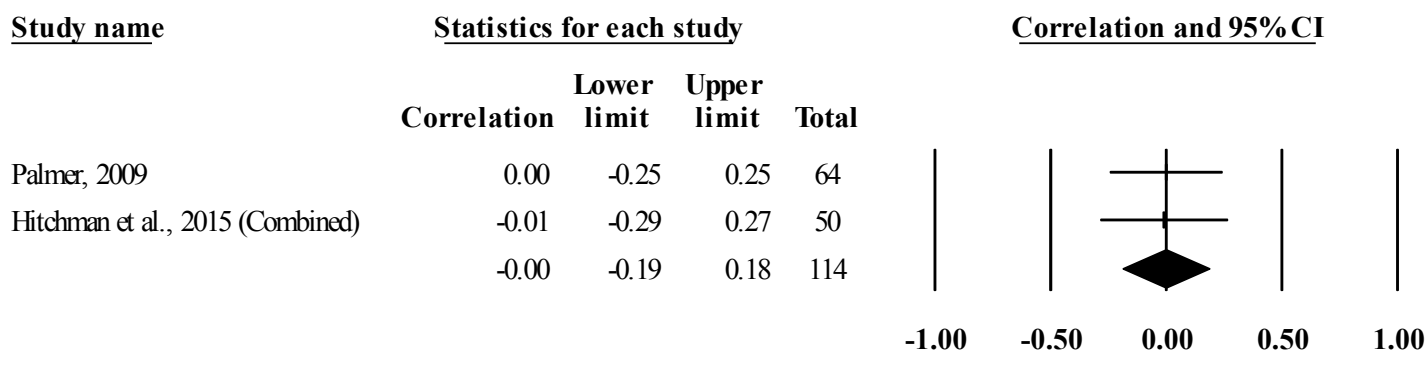

Figure 27. Meta-analysis of the relationship between temporal lobe dysfunction and psi performance in forced-choice precognition experiments.

4.4.10. Time belief as dynamic. "Time belief as dynamic" was reported in two studies by two independent investigators with a total of 14,016 participants and refers to how strongly one sees time as being in a constant flow. In both studies, Knapp and Garbutt's (1958) Time Metaphor Test was administered, which measures the belief that time is metaphorically "a dashing waterfall" or "a fast moving shuttle," for example. Figure 8 shows a forest plot of the correlation coefficients, with the correlations ranging from -.04 to -.02. The overall mean weighted effect size $(\boldsymbol{r})$ is -.02 ( $\boldsymbol{p}=.04$; 95\% CI [-.03, .001]), suggesting a small but significant negative relationship between time belief as dynamic and psi performance, such that people who do not view time as dynamic tend to perform better than those who see time as dynamic and hasty (see Figure 28). Furthermore, a test of heterogeneity was not significant $\left(\boldsymbol{Q}=0.05, \boldsymbol{p}=.82 ; \boldsymbol{I}^{2}=0 \%\right)$. The fail safe $\boldsymbol{N}$ was not calculated, as it is impractical to do so with less than three studies. 
Study name

Schmeidler, 1945 (Combined)

Schwartz \& De Mattei, 1983

$\begin{array}{rccc}\text { Correlation } & \begin{array}{c}\text { Lower } \\ \text { limit }\end{array} & \begin{array}{c}\text { Upper } \\ \text { limit }\end{array} & \text { Total } \\ -0.04 & -0.27 & 0.19 & 75 \\ -0.02 & -0.03 & -0.00 & 13941 \\ -0.02 & -0.03 & -0.00 & 14016\end{array}$

Correlation and $95 \% \mathrm{CI}$

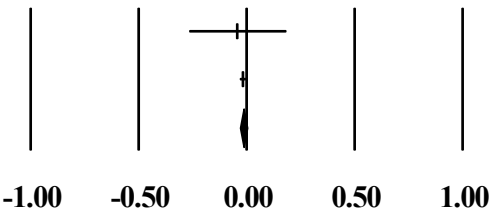

Figure 28. Meta-analysis of the relationship between time belief as dynamic and psi performance in forced-choice precognition experiments.

4.4.11. Future-orientation. Future-orientation refers to being more attentive towards future events than past events and was reported in two studies by two independent investigators based on a total of 118 participants. Both studies used the Attitude Toward the Future Questionnaire (Vaughan \& Houck, 1993). Figure 29 shows correlations of -.04 and .12, with an overall mean weighted effect size $(\boldsymbol{r})$ of .05 ( $\boldsymbol{p}=.57$; 95\% CI $[-.13, .23])$. A test of heterogeneity was not significant $(Q=.65, p=.42)$, with an $I^{2}$ of $0 \%$.

Study name

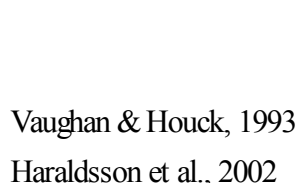

Statistics for each study

$\begin{array}{rccc}\text { Correlation } & \begin{array}{c}\text { Lower } \\ \text { limit }\end{array} & \begin{array}{c}\text { Upper } \\ \text { limit }\end{array} & \text { Total } \\ 0.12 & -0.12 & 0.35 & 68 \\ -0.04 & -0.31 & 0.24 & 50 \\ 0.05 & -0.13 & 0.23 & 118\end{array}$

Correlation and $95 \% \mathrm{CI}$
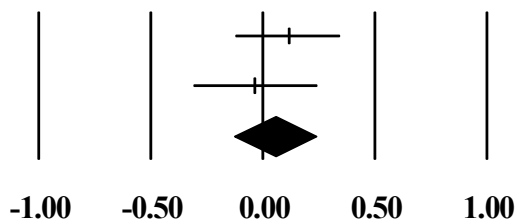

Figure 29. Meta-analysis of the relationship between future-orientation and psi performance in forced-choice precognition experiments.

4.4.12. Intelligence. Intelligence was measured in two studies by two independent investigators with a total of 80 participants. Both studies included only child participants, ranging in age from 4 to 14 . The measurement tools 
used were the Peabody Picture Vocabulary Test (Dunn \& Hottel, 1961) and a formal mathematical ability test using basic addition, subtraction, multiplication, and division problems. Figure 30 shows correlations of -.23 and .04 , with an overall mean weighted effect size $(r)$ of -.07 ( $p=.62$; 95\% CI [$.31, .19])$. A test of heterogeneity was not significant $(\boldsymbol{Q}=1.2, \boldsymbol{p}=.27)$, and the $I^{2}$ is $19 \%$.

Study name

Drucker et al., 1977

Winkelman, 1981 (Combined)

\begin{tabular}{rrrr}
\multicolumn{4}{c}{ Statistics for each study } \\
Correlation & $\begin{array}{c}\text { Lower } \\
\text { limit }\end{array}$ & $\begin{array}{c}\text { Upper } \\
\text { limit }\end{array}$ & Total \\
0.04 & -0.24 & 0.31 & 50 \\
-0.23 & -0.54 & 0.15 & 30 \\
-0.07 & -0.31 & 0.19 & 80
\end{tabular}

Correlation and $95 \% \mathrm{CI}$
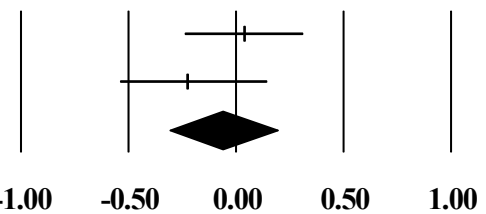

Figure 30. Meta-analysis of the relationship between intelligence and psi performance in forced-choice precognition experiments.

\subsection{Single Studies}

There were also a number of individual difference measures that were analysed only for a single study. As single studies cannot be meta-analysed, they will be presented individually in Table 1 below, in order of effect size. Of the twelve constructs assessed in relation to task performance, only 'memory' (in this case, how well one could remember details of a fictional tape recording) and optimism were significant (positive) predictors ( $\boldsymbol{r}$ 's $=.36$ and .20 , respectively). 
Table 1

Summary Statistics of All Individual Difference Measures Included in Only One Study

\begin{tabular}{llcc}
\hline Individual Difference Measure & Study Author(s) & Effect Size $(r)$ & $\begin{array}{c}\text { Sample } \\
\text { Size }\end{array}$ \\
\hline Memory & Stanford, 1970 & $.36^{*}$ & 30 \\
Sensitivity to Punishment & Hitchman et al., 2016 & -.21 & 51 \\
Optimism & Haraldsson et al., 2002 & $.20^{*}$ & 50 \\
Fantasy Proneness & Thalbourne, 1996 & -.13 & 99 \\
Latent Inhibition & Hitchman, Sherwood, \& Roe, & -.11 & 48 \\
Belief in the Occult & 2015 & & \\
Time Belief as Naturalistic & Haraldsson et al., 2002 & .10 & 50 \\
Psychotism & Schmeidler, 1964b & -.08 & 75 \\
Hypersensitivity & Haraldsson et al., 2002 & -.08 & 50 \\
Time Belief as Humanistic & Schalbourne, 1996 & .04 & 99 \\
Cerebral Lateralization & Palmer, 2009 1964b & .01 & 75 \\
Sensitivity to Reward & Hitchman et al., 2016 & .00 & 64 \\
\hline
\end{tabular}

$* p<.05$

\section{Summary of Results}

Below (Table 2) is a summary of all of the individual difference measures that were meta-analysed for comparison. The individual difference measures are ordered by overall absolute effect size (that is, by magnitude irrespective of direction).

Column 1 is the individual difference measure; Column 2 is the $95 \%$ confidence interval, ${ }^{5}$ Column 3 is the overall effect size; Column 4 is the number of individual studies included in the meta-analysis; Column 5 is the number of independent investigators in the meta-analysis; Column 6 is total number of participants for all of the studies in the meta-analysis combined.

\footnotetext{
${ }^{5}$ As effect size estimates based on previous research are inherently uncertain, confirmatory studies based on lower confidence intervals are less likely to overestimate the true effect size (Kennedy, 2016). Therefore $80 \%$ and $68 \%$ confidence intervals are provided in Appendix A, Table A2.
} 
Table 2

Summary Statistics of All Individual Difference Measures That Were Meta-Analysed

\begin{tabular}{|c|c|c|c|c|c|}
\hline $\begin{array}{l}\text { Individual } \\
\text { Difference Measure }\end{array}$ & $\begin{array}{c}95 \% \\
\text { Confidence } \\
\text { Intervals }\end{array}$ & $\begin{array}{l}\text { Overall } \\
\text { Effect } \\
\text { Size }(r)\end{array}$ & $\begin{array}{l}\text { Number } \\
\text { of } \\
\text { Studies }\end{array}$ & $\begin{array}{l}\text { Number of } \\
\text { Independent } \\
\text { Investigators }\end{array}$ & $\begin{array}{l}\text { Total Number } \\
\text { of } \\
\text { Participants }\end{array}$ \\
\hline Chance Belief & $-.09, .36$ & .141 & 5 & 1 & 248 \\
\hline Belief in ESP & $.07, .20$ & $.134 *$ & 24 & 12 & 2,250 \\
\hline Luck Belief & $.001, .26$ & $.131 *$ & 5 & 1 & 248 \\
\hline Perceptual & $.001, .24$ & $.125^{*}$ & 6 & 1 & 272 \\
\hline Defensiveness & & & & & \\
\hline Providence Belief & $-.03, .27$ & .125 & 5 & 1 & 248 \\
\hline $\begin{array}{l}\text { Openness to } \\
\text { Experience }\end{array}$ & $.03, .20$ & $.118 *$ & 11 & 5 & 572 \\
\hline Extraversion & $.01, .15$ & $.080^{*}$ & 14 & 7 & 1,206 \\
\hline Dream Recall & $-.04, .18$ & .070 & 4 & 3 & 799 \\
\hline Intelligence & $-.31, .19$ & -.065 & 2 & 2 & 80 \\
\hline $\begin{array}{l}\text { Emotional } \\
\text { Reactivity }\end{array}$ & $-.27, \quad .38$ & .064 & 3 & 1 & 151 \\
\hline Creativity & $-.04, .17$ & .063 & 10 & 3 & 546 \\
\hline Perceived Luckiness & $-.11, .23$ & .062 & 5 & 2 & 272 \\
\hline Conscientiousness & $-.09, .20$ & .056 & 3 & 3 & 187 \\
\hline Neuroticism & $-.08, .19$ & .054 & 9 & 7 & 528 \\
\hline Religiosity & $-.24, .14$ & -.054 & 2 & 2 & 149 \\
\hline Future-Oriented & $-.13, .23$ & .053 & 2 & 2 & 118 \\
\hline Fortune Belief & $-.10, .16$ & .032 & 5 & 1 & 248 \\
\hline Agreeableness & $-.09, .13$ & .021 & 7 & 4 & 556 \\
\hline Pro Attitude & $-.12, .08$ & -.019 & 3 & 1 & 393 \\
\hline $\begin{array}{l}\text { Time Belief as } \\
\text { Dynamic }\end{array}$ & $-.03,-.001$ & $-.017 *$ & 2 & 2 & 14,016 \\
\hline Transliminality & $-.13, .15$ & .008 & 5 & 1 & 542 \\
\hline $\begin{array}{l}\text { Spontaneous } \\
\text { Experiences }\end{array}$ & $-.08, \quad .07$ & -.006 & 3 & 2 & 695 \\
\hline $\begin{array}{l}\text { Temporal Lobe } \\
\text { Dysfunction }\end{array}$ & $-.19, .18$ & -.004 & 2 & 2 & 114 \\
\hline
\end{tabular}

\section{Discussion}

As we can see from the summary of results, the majority of these individual difference measures have not been extensively investigated, with the exception of belief in ESP, extraversion, and neuroticism. It might therefore be argued that such a meta-analysis is unnecessary. However, without a metaanalysis, researchers will likely impose their own synthesis of the data, and a meta-analysis can provide greater clarity in this regard-even if it only incorporates two or three studies, as Valentine, Pigott, and Rothstein (2010) argue that all other synthesis techniques are less transparent and/or less likely 
to be valid. At the same time, it is not intended to stop researchers from exploring individual difference measures that may not yet seem promising, especially those that have been tested only a handful of times; it is merely given as a benchmark of past results.

With that being said, the results suggest that there may be only a small pool of individual difference measures that are robustly correlated with performance on a forced-choice precognition task. This is also consistent with Steinkamp's (2005) review of forced-choice ESP experiments, where she found that "there are few variables which have correlated clearly with success ... most variables tested provided little evidence either way as being ultimately psiconducive and there were relatively few variables that appeared to be encouraging" (p. 155). However, notable exceptions in this meta-analysis include extraversion and belief in ESP, which show more consistent results across a larger number of studies. It should also be noted that with the number of meta-analyses conducted in this paper, there is an increased risk of familywise error, and that one or more of these significant findings might be the result of multiple analyses (e.g. represent a false positive). Further, while forced-choice ESP tests produce normal distributions (unless the number of trials is very small and/or the number of response alternatives is very large), it may be useful to incorporate nonparametric statistics in the case of any highly skewed distributions. Researchers should bear this in mind when setting their expectations for future experiments.

Nevertheless, the findings suggest a small but significant relationship between the following individual difference measures and psi performance: luck belief, perceptual defensiveness, openness to experience, belief in ESP, extraversion, and time belief as dynamic. If psi was an ability that could be learned (even if subconsciously), it is possible that these traits aid in the development of a more accurate precognitive ability. This is because the underlying mechanism of these traits (i.e. a combination of open-mindedness, curiosity, and intuition) might lead people to make more predictions (or act on 'hunches') in their day-to-day lives, which gives them relevant feedback to fine- 
tune their ability to make predictions. For example, people who report having strong luck belief (i.e. believing that they can control their own luck) would tend to have more confidence over what can and cannot happen to them, and consequently be more likely to make predictions about events in the future. Similarly, believers in ESP (of which precognition is a sub-component) are more likely to make predictions, given that they believe that precognition is possible. Those high in extraversion and openness to experience may also practice prediction-making, due to their curiosity and discussions with other people about what they believe the future holds. On the other hand, those who constantly act on 'hunches' are likely to score high in perceptual defensiveness (relating to preconscious processing), as they may be more intuitive individuals. Finally, those who believe that time is dynamic (i.e. believe that time is in a constant state of flow) may often ruminate about the direction of the future and therefore speculate on what is to come.

However, given that even the strongest predictor in this meta-analysis accounts for approximately only $2 \%$ of the variance on its own, perhaps these predictors are not related and are instead additive, and provide more power when analysed together. Therefore, the most optimal strategy for future researchers may be to combine individual difference factors, not just for the additive benefits but also to examine potential interactions (see Baron \& Kenny, 1986) between the factors that may predict precognitive performance.

Alternatively, these results may be due to statistical anomalies, having arisen from the large amount of analyses being conducted on individual differences in psi research, if not due just to methodological flaws. The other possibility is that the results reflect an actual relationship between certain variables combined with imperfect research designs. Taking into consideration the fact that many of these findings, including the non-significant results, were based on only a limited amount of studies, it is difficult to come to any strong conclusions. If one also considers the possibility of experimenter psi (i.e. where the experimenter influences the final results of an experiment due to his or her own psi abilities), it becomes extremely difficult to disentangle the data, 
especially in the case of a meta-analysis with only a single independent investigator. This potential explanation has previously been offered for the relationship between perceptual defensiveness and psi performance (see Haraldsson et al., 2002).

Retrospective meta-analyses also have several limitations, so it is not appropriate to make any definitive statements about the results without first conducting confirmatory studies. One such limitation is that included studies are often affected by publication bias or the file-drawer effect, whereby only significant results are reported or published. Although parapsychological journals generally publish more non-significant results than most mainstream scientific journals (Mousseau, 2003), no field is entirely immune, especially when there may be tens or even hundreds of secondary analyses conducted (e.g. various individual difference measures). Indeed, the low fail safe $N$ numbers found in several of these meta-analyses (e.g. nine for extraversion) suggest that publication bias is a possibility. At the same time, there is no indication to argue strongly that publication bias is a problem when taking into account Egger's test results and the funnel plots, which should be reassuring for parapsychologists given Mousseau's (2003) findings. Secondly, there will always be subjectivity involved in meta-analytical procedures and interpretation, such as defining and judging exclusion criteria, using search strategies, or coding the studies (Murray, 2011). Biases will come into playwhether conscious or unconscious-that influence procedural decisions, especially since researchers will already be aware of the results of the individual studies (Watt \& Kennedy, 2017). In fact, Gutierrez (2012) showed that three different meta-analyses, using the exact same studies and data, could still report wildly different outcomes (e.g. overall reduction of recidivism in one was $7.2 \%$ compared to $12.5 \%$ in another). This inherent subjectivity allows psi proponents and critics to conduct meta-analyses whose conclusions often support their own prior beliefs, but never manages to convince the other side (Palmer, 2003). 
Yet meta-analyses are still useful in that they can suggest the conditions under which replication is most likely to occur (assuming an effect exists). An overall effect size also gives future researchers the ability to calculate how many participants they would need to include in their experiment for it to be adequately powered. This is important, as many researchers are unaware of the importance of power and effect size, which has led to erroneous conclusions about the lack of replicability in many areas of parapsychology (Utts, 1991). With these key pieces of information, prospective meta-analyses (which define the exclusion criteria and other details beforehand) can then be conducted using only future studies that are to be pre-registered and fit the criteria. A prospective meta-analysis therefore avoids all the potential issues of publication bias and subjectivity that are evident in a retrospective meta-analysis, while also addressing methodological issues such as optional stopping. In fact, Watt (2016a) has set up a registration-based prospective meta-analysis of one of the most thorough yet controversial paradigms in parapsychology, the GanzfeldWatt's (2016a) meta-analysis protocol specifically includes only pre-registered individual studies that prospectively fit their criteria. This is a positive direction for parapsychology, as it brings structure and focus to the field.

Pre-registration has been made even easier by the Koestler Parapsychology Unit (KPU) registry, an initiative started at the University of Edinburgh in 2012, that allows researchers to prospectively register their experiments in detail, publically, and is not affiliated with a specific journal (Watt \& Kennedy, 2015). Not only are prospective meta-analyses the ideal way to test the replicability of psi phenomena, but they are also the best way to confirm the null hypothesis should psi not exist. Alcock (2003) claims that the latter hypothesis often does not get serious consideration by parapsychologists, so multiple prospective meta-analyses showing non-significance may force parapsychologists to give the null hypothesis more deliberation than a single study or retrospective meta-analysis would.

Another consideration for attempting replication is the researcher conducting the experiment. Although some parapsychologists argue that the psi 
experimenter effect eliminates the possibility of true replication, that is, that due to the nature of psi only experimenters who are proponents of psi will get positive results in psi experiments whereas sceptics will not (and there is some evidence of this, see Wiseman \& Schlitz, 1997), most researchers would only be satisfied that psi phenomena exist if it were to be consistently demonstrated by neutral scientists and not just a select few who believe in psi (Alcock, 2003; Hansel, 1966, 1980; Palmer, 2016). The current meta-analysis was conducted with this goal in mind, as forced-choice precognition experiments are arguably the easiest to run and can be automated using computer programmes. For example, Bem (2011a) conducted his Precognitive Detection of Erotic Stimuli experiment using an automated computer programme. This allows researchers to collect large amounts of data with relatively little effort, an important consideration if researchers are to try and replicate the small effect sizes shown in this meta-analysis (Steinkamp, 2005).

Ultimately, it is hoped that this meta-analysis can be used as a springboard for future research, allowing the findings to be used in a productive way and perhaps aiding in the development of research programmes that are specific and structured. As Watt (2005) comments, "[p]arapsychologists need to be far more systematic in how they tackle these questions. ... Systematic follow-up is an essential prerequisite for demonstrating a replicable effect" (p. 222). Research in parapsychology tends to have a shifting database, where an experimental paradigm remains popular for one generation of researchers only to be replaced with a new paradigm by the next generation (Hyman, 1994). Also, what constitutes evidence for psi seems to be constantly changing (rather than progressing) and may explain why parapsychology has still not achieved the recognition it desires from the mainstream scientific community (Hyman, 1994). With parapsychology being such a small field, it is important that researchers work together to build up a body of evidence that is considered respectable by both parapsychologists and mainstream academics. With the recent failures to replicate many foundational studies in both psychology and medicine (Open Science Collaboration et al., 
2015), now is the perfect time to define what a replicable psi experiment really is and take advantage of the benefits of pre-registration. Only then will we be able to finally confirm or disconfirm some of the major hypotheses in psi research. Depending on whether you are extraverted or believe in psi, you may already know how it will turn out. For those without psi ability, we turn now to the next chapter, where we examine some of these individual differences in our own set of studies. 


\section{CHAPTER FOUR: CONFIRMATION}

Chapter Three's meta-analysis uncovered six significant individual difference predictors of psi performance in the forced-choice precognition domain, spanning almost a century of published research.

The next step is to examine these predictors using a previously successful (and contemporary) forced-choice precognition paradigm—one that satisfies the following two criteria:

(a) The experimental paradigm has been published in a high-impact mainstream journal (this is not because parapsychological journals are less credible than mainstream journals, but rather, mainstream researchers are highly critical of parapsychology, so any paradigm that can pass the criticalness of mainstream researchers are likely to be highly robust), and

(b) The least susceptible to sensory leakage (i.e. automated, safeguarded against cheating, target generated after participant makes their choice).

In this aim, we turn to the work of Daryl Bem-a notable social psychologist and professor emeritus at Cornell University, who recently published a series of nine precognition experiments that we will use as the foundation for Study II of this thesis.

\section{Introduction}

In 2011, a paper by Daryl Bem (2011a) published in the Journal of Personality and Social Psychology (JPSP; a leading journal in the field of social psychology) brought parapsychology into the limelight. Bem (2011a) conducted nine experiments in total, eight of which were significant and purported to show a precognitive or retrocausal effect; that is, information in the future seemed to influence participants' choices in the present. While parapsychological research has previously appeared in mainstream journals before (see Bem \& Honorton, 1994; Targ \& Puthoff, 1974; Tart et al., 1980), 
Bem's latest paper had more of an impact, as he used well-established psychological paradigms such as priming and simply time-reversed them (e.g. priming words were displayed after the participant made their choice, not before), yet still managed to find similar effects. Overall, Bem's (2011a) research for this paper spanned 10 years, involved 1,050 participants, and had a mean effect size $(\boldsymbol{d})$ of 0.22 (with a Stouffer $Z$ of $6.66, \mathrm{p}=2.68 \times 10^{-11}$; Bem, Utts, \& Johnson, 2011).

Bem categorised his experiments into the following four standard psychological effects being tested: (a) approach/avoidance (b) affective priming (c) habituation (d) facilitation of recall.

\subsection{Approach/Avoidance}

Experiments 1 and 2, the two precognitive approach and avoidance experiments, are based on the idea that we are more likely to repeat a response that has been reinforced in the past compared to one that has not been reinforced (Lattal, 1998; Thorndike, 1898). Bem's (2011a) time-reversed version analysed this effect by looking at whether participants were more likely to predict an image that would be reinforced in the future. In Experiment 1, two curtains were placed side-by-side on a computer screen, and participants were invited to guess which side contained an image. After they made their choice, the computer randomly determined whether they were correct (which would constitute a 'hit'), and if they were, it displayed an image ('reinforcing' images were erotic in content, while neutral stimuli were non-erotic images). Across 100 sessions, participants correctly identified the erotic pictures significantly more than the $50 \%$ hit rate expected by chance: $53.1 \%, \boldsymbol{t}(99)=$ $2.51, p=.01$. In Experiment 2, the same procedure occurred but with the use of negative (i.e. gruesome and unpleasant) images rather than erotic images, such that correctly avoiding these images (i.e. choosing the opposite side that did not contain an image) would be considered a hit instead. The results similarly revealed a greater hit rate than (the 50\%) expected by chance: $51.7 \%$, $t(149)=2.39, p=.01$ 


\subsection{Affective Priming}

The two retroactive priming experiments ('retroactive' is used by Bem to denote the future actually causing the present action) were based on priming experiments that are regularly conducted in social psychology (see Klauer \& Musch, 2003). In a standard priming experiment, participants are asked to decide whether an image is pleasant or not, and their response time is measured. Before each image, a priming word is flashed, and the word can be either positive or negative (e.g. "gorgeous" or "disgusting"). A priming effect is considered to have occurred when reaction times are faster when the valences of both the primed word and the image are congruent (i.e. both positive, or both negative) as opposed to incongruent. Thus, in Bem's (2011a) Experiments 3 and 4, participants first had to decide the valence of an image and then a randomly selected prime was shown, with the hypothesis being that congruent priming words would elicit faster reaction times, even though these words appeared after the image. Indeed this effect was found in both experiments, with participants in Experiment 3 being 15 milliseconds faster on congruent trials than on incongruent trials, $\boldsymbol{t}(96)=2.55, \boldsymbol{p}=.006$, while participants in Experiment 4 were 27.4 milliseconds faster on congruent trials than incongruent trials, $\boldsymbol{t}(98)=2.03, \boldsymbol{p}=.02$

\subsection{Habituation}

Bem (2011a) describes his next set of experiments 5 through 7 as retroactive habituation. Habituation is the idea that people exposed to an emotionally arousing stimulus will have a strong physiological response to it, which then diminishes after continued exposure to the same stimulus. Such habituation may explain why after initially being scared of a frightening stimulus, one will become less scared of the same stimulus if repeatedly exposed to it. Similarly, if one is exposed to something positive, repeated exposure may lead to less arousal and consequently more boredom; or as the proverb goes, 'familiarity breeds contempt' (Dijksterhuis \& Smith, 2002). In Experiments 5 and 6, participants chose their preferred of two images (the 
images were displayed side-by-side and paired for equal likeability); each pair of images were either (a) both negative, or (b) both erotic. After the participant made their choice, the computer randomly determined one of the images to be the habituation target and flashed it subliminally on the screen several times (if they had correctly chosen it). The hypothesis was that participants would be more likely to prefer the habituation target if the images were both negative (since they would have been habituated to it), whereas they would prefer the non-habituation target if the images were both erotic (since their arousal should be higher with less habituation). Both experiments supported this hypothesis; participants in Experiment 5 preferred the target over the nontarget when they were negative picture pairs $(53.1 \%, t(99)=2.23, \boldsymbol{p}=.01)$, as did participants in Experiment $6(51.8 \%, \boldsymbol{t}(149)=1.80, \boldsymbol{p}=.04)$. For the erotic picture pairs-examined only in Experiment 6-participants preferred the target significantly less frequently than the non-target $(48.2 \%, t(149)=$ $1.77, \boldsymbol{p}=.04)$. Experiment 7 used the same procedure but specifically tested whether neutral images could induce boredom through habituation. However, the hit rate in this experiment was not significantly different from chance $(49.1 \%, t(199)==1.31, p=.10)$.

\subsection{Facilitation of Recall}

The final two experiments in Bem's (2011a) study investigated retroactive facilitation of recall by attempting to invert the phenomenon that practicing or rehearsing a set of verbal items will increase facilitation of recall afterwards. In Experiments' 8 and 9, participants were shown 48 words one-byone on a computer screen. They were then asked to recall as many words as they could remember. After participants had typed all of the words that they could remember, the computer chose half of the words as 'practice words' (the words selected were randomly determined) and participants were then asked to rehearse those words. The idea was that the words that were later practiced would influence the current decision, such that one would be more likely to recall the words that were later practiced compared to the other non-practiced words. The results supported this hypothesis, with Experiment 8 finding a 
significant DR\% of $2.27 \%, \boldsymbol{t}(99)=1.92, \boldsymbol{p}=.029$ (a positive DR\% indicates more practice words being recalled than control words), while Experiment 9 also found a significant effect: DR\% of $4.21 \%, t(49)=2.96, p=.002$.

Study II of this thesis will attempt to replicate Experiment 1 of Bem's (2011a) paper-the precognitive detection of erotic stimuli-as it also follows the same forced-choice precognition paradigm that we are looking to investigate. For a further discussion on the rationale of Study II, see section 5 .

\section{Controversy}

Soon after Bem's (2011a) paper was released-in fact, even earlier, as a pre-publication version of the paper enjoyed wide circulation beforehand (Carey, 2011)—critics from a variety of scientific disciplines attacked Bem's methodology, choice of subject matter, JPSP for allowing publication, and psychology as a field. Some commentators claim that this paper single-handedly led to the reformation of the field of psychology and scientific practices in general. This is because (some) people see Bem's results as implausible (i.e. a false positive) even though the methodological practices he used are common to science, which implies that the entire field needs reformation (as there are likely to be plenty more false positives incorporating the same inadequate yet currently accepted methodological practices: see section 4). It is appropriate, therefore, to consider in further detail the criticisms and rebuttals to Bem's (2011a) paper, to give us a greater understanding of its strengths and weaknesses, and its impact on the scientific world.

\subsection{Methodological Criticisms}

Yarkoni (2011) argues that there was no critical flaw in Bem's (2011a) paper that rendered it a 'bad' paper per se; rather, there were numerous minor, methodological flaws that collectively lead to a conclusion that could not be supported. The first of these issues is that across the nine experiments, the number of participants per experiment varied greatly (i.e. they were not consistent with one another). Instead, each individual study contained 
anywhere from 50, 100, 150, or 200 participants, an inconsistency that was never explained in the article. Yarkoni (2011) argues that while one can only speculate as to why this is the case, in the absence of a priori power analyses the main reason sample sizes vary within a paper is due to data peeking (i.e. looking at your data and then deciding to continue the study or stopping it right there). While Bem was not completely indifferent about this as his sample sizes were still in chunks of 50, the probability of a Type I error, or false positive, would still have been increased if data peeking had occurred. Furthermore, Yarkoni (2011) is critical of the fact that most of Bem's (2011a) experiments reported $p$-values that were very close to .05 , whereas you should expect to see at least some strongly significant $\boldsymbol{p}$-values amongst the nine studies (assuming there is an effect). This lends credence to some sort of selection bias. In addition, Alcock (2011) found a large negative correlation between both (a) effect size and sample size, $\boldsymbol{r}=-.91$, and (b) effect size and power, $\boldsymbol{r}=-.80$, so optional stopping seems to be a plausible explanation (Ritchie et al., 2012; Schimmack, 2012).

There is also a corollary to this: Bem's (2011a) experiments are not highly powered so, based on random variability, we would not expect to see so many significant results in one paper even if the effect were real. As Francis (2012) put it,

If all of the experiments have high power (the probability of rejecting the null hypothesis when it is false), multiple experiments that reject the null hypothesis would indeed be strong evidence for an effect. However, if the experiments have low or moderate power, then even if the effect were real, one would expect to frequently not reject the null hypothesis." (p. 152)

It is speculated, therefore, that either Bem did not report all of the relevant information or he ran his experiments incorrectly, since the null hypothesis was rejected too often for the results to be plausible (Francis, 2012). 
The form in which the studies were presented in the final publication, may also not reflect Bem's original intentions and design. Yarkoni (2011) suggests that some experiments were either split or combined post-hoc, depending on the analysis of the data. For example, Bem slightly modified the design of Experiment 2 partway through the experiment (the sequence of images differed for the last 50 participants), yet all 150 participants were combined as one. In contrast, Experiments' 8 and 9 were split into two different experiments of 100 and 50 participants respectively (also due to slight experimental modification). The fact that these similar design adjustments were treated differently invites speculation that Bem lumped together all participants in Experiment 2 due to the results of the last 50 participants failing to reach statistical significance (but combining them preserved the overall significance). Again, such a practice would inflate the risk of a Type I error, since these adjustments occur post-hoc and are not part of the original hypothesis. Furthermore, it is unclear how many experiments Bem ran in total, given that Bem omitted three sets of findings (the details of which are rather vague in his paper-although to his credit, other researchers may not have even mentioned them at all). It would be much more informative for Bem (2011a) to report exactly how many experiments and participants he ran in total, including any pilot sessions (Schimmack, 2012; Yarkoni, 2011).

Experiment 1 is also a case in point, as Bem (2011a) modified the procedure partway through the experiment, with the first forty participants being shown 36 pictures equally split between erotic, negative, or neutral pictures, whereas the last sixty participants only included erotic and non-erotic positive images "with both high and low arousal ratings" (Bem, 2011a, p. 409). It is unclear why the participants were pooled together with such a direct change in procedure, and what was meant by high or low arousal ratings seems ambiguous (Alcock, 2011). Furthermore, Bem states that in all experiments that used erotic or negative stimuli, a large number of non-arousing trials were included to reduce the participant's arousal level back to normal between the trials. However, Bem does not state the number of non-arousing trials (and how 
this number was determined), which makes the entire experimental procedure rather ambiguous and leaves us with more questions (Alcock, 2011).

Another reason to be cautious of Bem's (2011a) findings is that some of his hypotheses do not seem justifiable. Most notably, in Experiment 1, Bem's hypothesis is that people should be able to predict erotic images in advance but not any other type of image, due to evolutionary adaptations that are essential for survival and reproduction. However, Yarkoni (2011) argues that this reasoning is far-fetched (e.g. why only erotic images?), while Alcock (2011) states that no effort was made to determine whether erotic images indeed reinforced anything. One could easily make the argument that it is also adaptive to predict other potentially positive signals in the future, which includes more than just digital still images of erotica. Therefore, if results associated with any of the other five image types happened to be statistically significant, Bem could have justified those results post-hoc also (Schimmack, 2012). This is known as the 'garden of forking paths', where any outcome can be made to look consistent with hypotheses (e.g. throw a dart against a wall, and you can then draw a bullseye on where it lands) when no outcome is prespecified beforehand (Gelman, 2017). Further evidence of this is the fact that Experiments' 8 and 9 also used neutral stimuli as part of their hypotheses, even though neutral stimuli were specifically excluded in Experiment 1's hypothesis and were instead delegated to the control condition (Rouder \& Morey, 2011). Thus, Bem should have corrected his $\boldsymbol{p}$-values for this exploration of data (Wagenmakers, Wetzels, Borsboom, \& Van Der Maas, 2011).

To be clear, there is nothing wrong with exploring data and creatively searching for a potential effect. However, such post-hoc analyses needs to be distinctly labeled as exploratory and the statistics need to be adjusted for multiple analyses (Wagenmakers et al., 2011; Watt \& Kennedy, 2015). There appears to be further exploratory analyses that were not mentioned, since Bem stated that a variety of individual difference measures (that were nonsignificant) in pilot experiments were omitted in the paper, yet he still reported a gender effect in Experiment 5, which suggests he was looking at some 
moderators in his other experiments without accounting for multiple analyses (Wagenmakers et al., 2011). It is not entirely clear which analyses were exploratory or confirmatory, but overall-from the information we do havewe know that multiple testing did occur, and that it was not adjusted for statistically. Therefore, even if we assume that the actual experiment was rigorously conducted, none of the results are significant once we adjust for multiple analyses (Alcock, 2011).

Critics have also taken aim at Bem's (2011a) nonchalant use of onetailed tests. While some might take the position that one-tailed tests are never appropriate (e.g. Eysenck, 1960), most researchers would not take such an extreme approach while at least agreeing that you need a truly directional hypothesis if you are to use them. In this instance, Yarkoni (2011) argues that it is very unlikely that Bem's hypotheses are directional, given the varied ways in which psi can manifest itself (for example psi-missing, which is the ability to score below chance expectation) and the fact that these time-reversed experiments are all novel. Therefore, Bem's (2011a) use of one-tailed tests does not seem well-justified.

Ultimately, when it comes to methodological criticisms of Bem's (2011a) paper, we do need to acknowledge that we are making a few assumptions, since journal publications rarely contain every single step and procedure that took place (Francis, 2012; Yarkoni, 2011). Nevertheless, there are actions Bem could have taken even after its publication to address and alleviate some of these concerns, which he has not done (Yarkoni, 2011).

\subsection{Criticism of Parapsychology}

Another criticism leveled not specifically at Bem (2011a), but at parapsychology more generally, is that parapsychologists should change the statistical methods they use to determine the potential existence of psi phenomena. Schimmack (2012) argues that "[f]or controversial or very important research findings, the significance level could be set to $\boldsymbol{p}<.001$ or, 
as in particle physics, to $p<.0000005$ " (p. 8). Coover (1917), one of the first Americans to use statistical methods in parapsychology, similarly argued that the $\boldsymbol{p}$-value needs to be less than .0000221 at the very largest, for us to be confident that the results are indicative of phenomena other than chance (calculated using an application of Bayes Theorem, known as the Theory of Poisson). Others researchers argue that Bem's (2011a) paper shows us the weaknesses of frequentist methods (i.e. Null Hypothesis Significance Testing; NHST) in research and we should move towards using Bayesian methods instead (Wagenmakers et al., 2011). As Wilson and Shadish (2006) note, NHST is practically invulnerable to falsification as one is never able to show that the null hypothesis is true. In a field like parapsychology, the extension of theory could go on forever using NHST (in the same way that you could test any unsubstantiated hypothesis ad infinitum, such as whether tulips will react in some way to the sound of musical instruments), which may not be the most productive use of time or resources (D. B. Wilson \& Shadish, 2006). Using Bayesian methods, however, one can quantify the evidence in favour of the null hypothesis, giving the null hypothesis more weight than in NHST. Wagenmakers et al. (2011) re-analysed Bem's (2011a) data using Bayesian methods and found that of the 10 critical tests presented in his paper, six were weak (i.e. the results should not sway a critical researcher one way or the other), one was in favour of psi, and three were in favour of the null hypothesis. So overall, the evidence for a precognitive effect is not supported once Bayesian methods are used.

However, the strongest reason to doubt Bem's (2011a) reported results are the failed replication attempts. For example, Galak, Leboeuf, Nelson, and Simmons (2012) attempted to replicate Experiments' 8 and 9 (recall facilitation) by conducting seven of their own experiments across seven different samples, using over 3000 participants. All except one of their results were non-significant, and a meta-analysis of these seven experiments along with all other replication attempts in the retroactive facilitation of recall paradigm showed an average effect size $(\boldsymbol{d}=0.04)$ no different to chance 
(Galak et al., 2012). Similarly, Ritchie et al. (2012) conducted 3 independent and pre-registered replications of Bem's Experiment 9, which was also nonsignificant ( $\boldsymbol{p}=.83$ ). While some critics (e.g. Alcock, 2011) take it one step further and claim that JPSP should never have published the paper at all, not all critics agree with such a hardline stance, with French (2012) warning that "[o]nce we think we know in advance which effects are real and which are illusory, true scientific objectivity flies out of the window" (para. 7).

\section{Responses to the Criticism}

Bem (2011b) addresses the methodological criticisms by arguing-in response to Alcock's (2011) comments specifically—that most of these criticisms do not "distinguish between potential flaws in an experiment that would illegitimately produce false positive results and potential flaws that would actually work against the experimental hypothesis by introducing noise into the data" (para. 9). Bem (2011b) argues that most of the methodological issues raised fit in the latter category and would thus, if true, have simply caused more non-significant results (and even type II errors) rather than accounting for purported false positives (type I errors). However, this does not address the methodological concerns relating to multiple analyses of (presumably) exploratory testing/unreported experiments. Bem (2011b) did argue that the criticisms of Experiment 1 (i.e. that he did not adjust for any of

the five image types which could have been significant and led to different posthoc hypotheses) were irrelevant, as statistically adjusting for the other four image types would have produced the same conclusion (i.e. that they are all non-significant). However, this is not a compelling response, as adjusting for these exploratory tests would also mean that Bem would have had to adjust the findings for erotic images, which would render the finding non-significant. Nevertheless, the rest of Bem's (2011b) responses relate to his use of different statistical tests on the same data, which he says does not need to be accounted for statistically since its purpose is to show that the same conclusions can be reached no matter which statistical test was used. Again, these are not the multiple analyses that critics such as Yarkoni (2011) and Schimmack (2012) 
are referring to when pointing out Bem's methodological flaws; Bem does not address any of these specific concerns directly.

In response to Wagenmakers et al.'s (2011) claim that using Bayesian methods shows (a) the weakness of frequentist methods and that (b) Bem's (2011a) findings were not actually supported, Bem, Utts, and Johnson (2011) counter that Wagenmakers et al. (2011) failed to apply Bayesian methods correctly. In order to pit the null hypothesis against an alternative hypothesis, one must first specify both (a) the prior odds that the null hypothesis is true compared to the alternative, and (b) the probability distribution of the potential effect sizes for both the null (which would be zero) and the alternative hypothesis. For (a) the prior odds that the null hypothesis is true compared to the alternative, Wagenmakers et al. (2011) formally expressed the prior odds as 99,999,999,999,999,999,999 to 1 , which Bem et al. (2011) argue is unrealistic, as it makes proving psi almost impossible. Furthermore, regarding (b), which is the distribution range of the effect size if the alternative hypothesis is true, Bem et al. (2011) argue that Wagenmakers et al.'s (2011) decision not to specify a range (which means it defaults to a range of 0 to 1 ) was unjustified, as there are relevant pieces of information that can be used to narrow down the range at least to some degree. For example, based on past psychological research in general, most effect sizes range between .20 and .30 , and combined with the fact that no reasonable person would expect to see effect sizes above .80 for psi phenomena (as that would be so obvious that we would not even be testing it), you could at least limit the maximum range to .50 (Bem et al., 2011). Without such knowledge-based range adjustments, the probability increases "that any observed data will be higher under the null hypothesis than under the alternative" (Bem et al., 2011, p. 717). If we adjust the distribution to a more realistic prior (e.g. an effect size range between 0 and .50), the Bayesian analysis reveals the same conclusions as Bem's (2011a) original frequentist analysis (Bem et al., 2011).

In response to the failed replication attempts, Schimmack (2012) argues that although Ritchie et al. (2012) replicated the same procedure and number 
of participants that Bem used for his original Experiment 9, Bem's experiment was severely underpowered to begin with, so running the same number of participants does not tell us much. Furthermore, Bem (2012) claims that Ritchie et al. (2012) failed to include two more pre-registered studies in their analysis (both of which were statistically significant and pre-registered on the same registry used by Ritchie and colleagues), so it is premature to claim that an effect is not replicable after just a few replication attempts. Bem et al. (2015) also provide evidence that the effect is indeed replicable, having conducted a meta-analysis of all of Bem's (2011a) original experiments and consequent replication attempts, which spans 90 experiments (including Bem's original experiments), 33 laboratories, and 14 countries, with an overall effect greater than 6 sigma $\left(\boldsymbol{z}=6.40, \boldsymbol{p}=1.2 \times 10^{-10}\right)$ and an effect size (Hedges' $\boldsymbol{g}$ ) of 0.09. However, Lakens (2015) has criticised this meta-analysis for selectively including and precluding studies based on a protocol that was not clearly defined ahead of time, along with a lack of pre-registered replications (which introduce similar problems as Bem's original experiments, such as multiple testing/increased researcher degrees of freedom). These are the same limitations discussed in Chapter Three regarding retrospective meta-analyses. Furthermore, Lakens (2015) claims that Bem et al.'s (2015) publication bias analyses were incorrect, and when an appropriate PET-PEESE meta-regression is properly applied, the overall effect size is reduced to .0008 (with a confidence interval that includes zero), no different to chance.

\section{Replication Crisis}

However, many commentators instead chose to use Bem's (2011a) paper as a springboard to highlight inappropriate methodological practices of psychology and the wider scientific community in general (see Wagenmakers et al., 2011). As Rouder and Morey (2011) note, "when seemingly implausible claims are made with conventional methods, it provides an ideal moment to reexamine these methods" (p. 682). These commentators would argue that there is not necessarily a fault in Bem's specific methodology or paper, but rather, there is a fault with the system that can allow a scientific journal to 
publish findings that are so obviously 'false' (in the opinion of the critics).

For example, Francis (2012) looked at the number of null hypothesis rejections in experimental psychology and found rates very high (e.g. 97.3\%; Sterling, 1959, and 95.56\%; Sterling, Rosenbaum, \& Weinkam, 1995), so one would assume that the statistical power of these same experiments were also in the range of .90 and above. However, Hedges (1984) showed that the power in psychology experiments are typically much lower (within the range of .25 to .85), so there should be more findings that support the null hypothesis even if the effect is real (just by chance). In fact, Ioannidis (2005) came to this conclusion over a decade ago, when he calculated that the positive predicted value (PPV, or a paper's likelihood of presenting true results based on power, bias, and ratio of true to not-true relationships) in most published papers were often under $50 \%$, so there are likely many false positives in the scientific literature (and not just psychology). Moving forward, Ioannidis (2005) argues that we should aim to maximise PPV by conducting studies that have (a) larger sample sizes (b) larger effect sizes (c) a smaller number of previously tested relationships (d) less flexible designs, definitions, and outcomes (e) less financial interests and prejudices involved, and (f) less scientific teams working on the same problem, since more teams working on a single issue tend to lead to shortcut-taking due to its competitiveness.

To understand how these false positives come about, Wagenmakers et al. (2011) state that it is useful to look at Bem's (2011a) publication as a case study for the entire scientific domain, showing how statistics can be misused to mislead people. They go on to argue that "[t]he field of psychology currently uses methodological and statistical strategies that are too weak, too malleable, and offer far too many opportunities for researchers to befuddle themselves and their peers" (Wagenmakers et al., 2011, p. 426). Simmons, Nelson, and Simonsohn (2011) suggest that there are so many 'researcher degrees of freedom' to find an effect when running a study, that our $\boldsymbol{p}$-value threshold should be far lower than .05. For example, they showed that by working backwards and trying to show the nonsensical conclusion that listening to 
certain music can actually reduce one's age, they could support their a priori position as statistically significant by merely using standard justifications of ambiguous information commonly employed by researchers today (e.g. flexibility in sample size, the use of covariates, or reporting subsets of experimental conditions). In a similar vein, some commentators have put forth the idea that Bem intentionally published his paper in a top-tier journal precisely because he had an issue with the way research was being conducted in psychology and thought it would be the best way to bring more attention to the field (Gelman, 2013).

The aftermath of Bem's (2011a) paper led to many replication attempts, many of which have gone unpublished, and highlighted the publication bias prevalent in many psychology journals. For example, Ritchie et al.'s (2012) paper was initially rejected from many high-impact journals such as Psychological Science and even JPSP (the original journal that Bem's paper was published in, as they explicitly told them that they do not publish replication failures). Eventually JPSP did publish Galak et al.'s (2012) replication failure, but this was likely due to mounting pressure from the controversy Bem's paper had brought to the journal. The failure to consistently replicate Bem's (2011a) experiments also tied in to a greater debate on the replicability of psychology experiments in general. This was led by a large-scale replication project starting in November 2011, in which researchers attempted to replicate 100 classic psychology studies and found only 36 of the 100 replications to be statistically significant (Open Science Collaboration et al., 2015). All studies were high quality and highly powered according to strict protocols they followed, casting doubt on many other landmark studies conducted in psychology. Other disciplines, such as biomedicine and cancer research, have also entered the spotlight, with some replication rates as low as 11\% and most below 50\% (Begley \& Ellis, 2012; Osherovich, 2011; Prinz, Schlange, \& Asadullah, 2011). For these reasons, it is important we use this opportunity to fix the conventional research practices that have allowed these low reproducibility rates to thrive, as not doing so will have a devastating toll on the credibility of science 
(Schimmack, 2012; Simmons et al., 2011).

\subsection{Recommendations}

Wagenmakers, Wetzels, Borsboom, van der Maas, and Kievit (2012) argue that one way to reduce the amount of data "double-dipping strategies" that may lead to low reproducibility rates, is to make pre-registration compulsory for all experiments in psychology. Put another way, we need to specify beforehand which analyses are going to be conducted on the data and this information needs to be recorded somewhere for verification. This is standard practice in medical and pharmaceutical research, with the registry being public and openly accessible-in fact, a similar registry has been created specifically for parapsychological research (KPU Registry; Watt \& Kennedy, 2015). While some might argue that this is a draconian measure that stifles creativity (adding another bureaucratic layer), it does not actually prevent exploration of the data in the sense of being able to come up with new hypotheses; rather, it just forces researchers to distinguish between hypotheses and analyses that are predicted in advance (i.e. confirmatory analyses) and those that are done post-hoc (i.e. exploratory analyses) (Wagenmakers et al., 2012). As long as this process is transparent, all data and results can continue to be published and disseminated as is, without many practical changes (Bierman \& Bijl, 2014).

There are some difficulties with pre-registration, such as it potentially requiring too much paperwork, being too expensive, or being difficult to enforce in the psychological domain since most psychological research is exploratory (Francis, 2012). However, pre-registration may be a necessity, given that many researchers openly admit to questionable research practices (QRPs) such as testing their data in multiple ways to fit their hypothesis, which suggests that they may not even consider them questionable at all (John, Loewenstein, \& Prelec, 2012). Regardless of their intentions, researchers still suffer from the same confirmation and hindsight biases as the general population, so stringent criteria to follow for publication seems justified 
(Wagenmakers et al., 2012). This can be taken one step further by also requiring pre-registration of meta-analyses themselves (setting out specific coding and inclusion criteria beforehand), such that only prospective preregistered studies fitting these requirements shall be included in these metaanalyses (Akers, 1985; Kennedy, 2004; Milton, 1999; Watt, 2005). This is the only way to ensure that there are no QRPs, or bias, affecting the results of both the studies themselves, and the overall meta-analysis. Kennedy (2004) further suggests that we should have a committee in place that evaluates these metaanalytical proposals beforehand, to go over potential methodological issues that may arise before the data is collected so that they can be adequately refined.

Wagenmakers et al. (2012) also suggest that top journals (specifically, to set an example for all other journals) can help facilitate the transition to more confirmatory research by "implementing a policy to reward empirical manuscripts that feature at least one confirmatory experiment; for instance, these manuscripts could be published in a separate section explicitly containing confirmatory research" (p. 637). This also means that journals need to be more open to publishing null results, following the likes of the Journal of Parapsychology (which added a mandate to publish null results in 1980 well in advance of the replication crisis) and the Journal of Articles in Support of the Null Hypothesis (an online-only journal which publishes original experimental studies in all areas of psychology where the null hypothesis is supported). If this becomes mainstream, researchers will be less likely to engage in QRPs given that their work will receive more scrutiny by other researchers (e.g. researcher $\mathrm{X}$ may conduct the same experiment as researcher $\mathrm{Y}$ and find a null result, yet still get accepted in the same journal as researcher $Y$, thereby leading readers to investigate why the results are different), while also incentivising rigorous replication studies if null results offer the potential for publication in a top-tier journal (Schimmack, 2012). We just need to be careful that this does not lead to an increase in false negatives, which Fiedler, Kutzner, and Krueger (2012) claim are equally (if not more) problematic, since they are much harder to notice or be corrected by other researchers. 
Another factor potentially contributing to reproducibility issues is that current journals tend to favour multiple studies over single studies, which is counterproductive as "[t]he strength of the empirical evidence is a function of the total sample size rather than the number of studies" (Schimmack, 2012, p. 8). In other words, researchers will likely perform far more QRPs to get the outcome they need on at least some of their experiments (that they intend to publish in a single paper) than if they had just conducted one large, highly powered, study (Schimmack, 2012). This is illustrated by the fact that five studies with $80 \%$ power should produce one non-significant result, yet journal editors might reject such findings in an attempt to uphold an inadequate notion of what 'true' results look like (Schimmack, 2012). We can help prevent such biases by emphasising $\boldsymbol{p}$-curve analyses. The $\boldsymbol{p}$-curve is the distribution of $\boldsymbol{p}$ values across a number of studies in a single paper, and can be analysed for its overall evidential value across all of the studies, since a 'true' overall effect will contain more low values (.01s) than high values (.04s) (Simonsohn, Nelson, \& Simmons, 2014). Along the same line, Schimmack (2012) developed the Incredibility Index (IC-Index) which calculates the probability that there should have been more non-significant results in a multiple-study article (i.e. if the ICIndex is high, it is highly likely that QRPs have taken place as there should have been at least one more non-significant finding in the set of findings, even if the effect is real). Both $\boldsymbol{p}$-curve analyses and the IC-Index are tools that greatly benefit journal editors, as it gives them a more even-handed way to filter manuscripts that are likely to have used QRPs (it also benefits researchers who wish to analyse the effect of publication bias on published findings). Given that the editors of the JPSP stated that they took Bem's (2011a) data at face value and therefore had no other option but to publish it (Judd \& Gawronski, 2011), incorporating the IC-Index in the future may provide more objective measures that can help guide editors' decisions.

\section{Rationale for Study II}

At this point in time, it seems that the debate will continue and psi will not become an accepted phenomenon unless independent researchers can 
consistently replicate it. Given that the controversy over Bem's (2011a) paper centered largely over concerns that exploratory analyses were presented as preplanned hypotheses, replication attempts can address these concerns. Study II follows along this path, by using the same forced-choice precognition paradigm employed by Bem (2011a) in Experiment 1, while combining it with the most promising individual difference predictors (namely, belief in ESP, extraversion, and openness to experience, as we did not have access to all scales at the time of the experiment) that were found in the meta-analysis of Study I in this thesis.

We also included two exploratory individual differences, namely transliminality and magical ideation, as they have performed well in other parapsychological studies yet are less explored in the forced-choice precognition domain (see Parker, Grams, \& Pettersson, 1998; Thalbourne \& Storm, 2012). Specifically, transliminality is defined as a hypersensitivity to psychological material entering one's subconscious (Thalbourne \& Maltby, 2008) and has been shown to predict above-chance performance in laboratory clairvoyance experiments (e.g. Del Prete \& Tressoldi, 2005) and precognitive habituation experiments (e.g. Parker \& Sjödén, 2010). Its incorporation in these experiments is based on the idea that psi is a subliminal process and therefore people who are sensitive to material (i.e. imagery, ideation, affect, perception) originating in the subconscious and/or external environment are likely to have greater psi ability (Thalbourne \& Storm, 2012). Magical ideation is a related concept, defined as belief in forms of causation that by conventional standards are invalid, and is a common symptom in schizotypy or schizophrenia proneness (Eckblad \& Chapman, 1983). It has shown repeated success in predicting psi performance in Ganzfeld experiments (e.g. Parker et al., 1998).

Since Bem (personal communication, June 4, 2015) provided the resource materials for us to run an exact copy of his experiment, the experimental procedure was effectively fixed before we began. Pre-planned analyses were also documented in our ethics application (submitted to the ethics board at Victoria University of Wellington, and appendicised with this thesis) prior to running the experiment, which simultaneously acted as an ad- 
hoc pre-registration of the experiment (as the timestamp and contents can be independently verified).

Experiment 1 of Bem's (2011a) paper was chosen as the experimental paradigm of choice, as it uses a straightforward forced-choice precognition design with seemingly robust effects, while also having the advantage of containing a control condition where psi presumably cannot manifest itself (i.e. the non-erotic image conditions). This is rare, as researchers have been unable to reliably specify a condition in which psi does not exist, so it will be helpful to compare any successful outcome to that of the control condition to verify that psi is indeed operating as expected (e.g. if the control condition also contains significant results, then we can be less certain of any of the results). Bem (personal communication, June 4, 2015) also provided the resources for this experiment as other independent researchers have reported the most success replicating this experiment, compared to any of Bem's (2011a) other experiments.

The major goal of Study II is to provide a well-controlled and predictable demonstration of psi that can be replicated by independent investigators. Based on the findings of Study I, we hypothesise that (a) belief in ESP, (b) extraversion, and (c) openness to experience, will significantly correlate with performance on the psi task (the number of "hits" in the erotic images condition) in Study II.

\section{Method}

\subsection{Participants}

Two hundred and eight Victoria University of Wellington first year psychology students were recruited and received credit toward a research participation requirement. Of these participants, 135 were female and 73 were male. Recruitment was conducted via the School of Psychology's online sign up system. A two-tailed power analysis with $\boldsymbol{\beta}=.80$ and $\boldsymbol{r}=.08$ (based on the lowest overall effect size of the three individual difference factors being 
explored from Study I) confirmed the need for at least 198 participants. Therefore, 200 participants (rounded up from 198) were pre-specified on an Ethics Application form submitted to VUW before the experiment began, with the actual experiment recruiting slightly more than 200 participants so as to make sure we would get at least 200 participants' data (given that there are often no-shows and the recommendations for more, rather than fewer, participants).

\subsection{Materials}

A replication package (computer software) was received from Bem (2011a) of his first experiment, Precognitive Detection of Erotic Stimuli, following an email request to replicate one of his experiments. The images used in this package come from the International Affective Picture System (IAPS; Lang \& Greenwald, 1993), a set of 820 digitised photographs that have been rated on 9-point scales for valence and arousal by both male and female raters, and are commonly used in research on numerous topics. However, stronger and more explicit images were included from the internet for males, as Bem's (2011a) previous experiment showed different levels of arousal for males and females. Two additional sets of erotic images were included, so that men could choose the option of seeing male-male erotic images and women could choose the option of seeing female-female erotic images. Each session included both erotic and non-erotic images randomly intermixed, while the actual sequencing of the images along with their left/right positions were determined by the programming language's internal random function. As per Bem's (2011a) justification, the erotic images were used as specific reinforcement for correct "precognitive" guesses, whereas the non-erotic images were used as a control. The replication package also included a belief in ESP measure, with the following question: "Do you believe that ESP exists?" answered on a five-point Likert scale, ranging from 1 (definitely does not) to 5 (definitely does).

A questionnaire comprising the Ten Item Personality Inventory (TIPI; Gosling, Rentfrow, \& Swann, 2003), the Magical Ideation Scale (MIS; Eckblad 
\& Chapman, 1983), and the Revised Transliminality Scale (RTS; Lange, Thalbourne, Houran, \& Storm, 2000) was also administered to participants. This questionnaire had been compiled for use in a previous sheep-goat experiment at Victoria University of Wellington, so we decided to implement it in this study also. Although this questionnaire does not measure all of the significant predictors found in Study I (i.e. luck belief, perceptual defensiveness, time belief as dynamic), we did not have access to all of those scales at the time of the experiment, so these three predictors were omitted from analysis.

The TIPI contains ten items measured on a seven-point Likert scale ranging from 1 (strongly disagree) to 7 (strongly agree) and is a brief measure of the Big-Five personality dimensions of extraversion, agreeableness, conscientiousness, emotional stability, and openness to experience. Example items include "Extraverted, enthusiastic" to measure extraversion and "Open to new experiences, complex" to measure openness to experience. While not as extensive as other Big-Five measures, the TIPI shows test-retest reliability, along with convergent and discriminant validity (Gosling et al., 2003).

The MIS contains thirty true-false items that assess the magical ideation component of schizotypal personality, which is the belief in certain forms of causation that are by conventional standards invalid (Eckblad \& Chapman, 1983). Example items include "Some people can make me aware of them just by thinking of them" and "Horoscopes are right too often for it to be a coincidence".

The RTS is a 17-item true-false Rasch-scaled questionnaire that corrects for the age and gender biases of the previous 29-item Transliminality Scale, and measures the hypothesised tendency for psychological materials to cross thresholds into or out of consciousness (Lange et al., 2000). Some items included in the RTS are: "At times I perform certain little rituals to ward off negative influences" and "I have experienced an altered state of consciousness in which I felt that I became cosmically enlightened". 
Finally, there was a two-item stimulus seeking scale embedded in the replication package, having been developed by Bem (2011a).

\subsection{Procedure}

Participation took place in a computer laboratory equipped with 10 computers, and up to 10 people participated in each experimental session. As in Bem's (2011a) study, participants were given a consent form upon entering the laboratory, which informed them that they must be at least 18 years of age to participate, and included an explanation of the procedure (and that it tests for ESP) along with a disclaimer that the experiment contains erotic images. Participants were also made aware that they could leave the experiment (and still receive course credit) if they objected to the experiment at any time.

After signing the consent form, participants were seated in front of a computer. A relaxation period followed, in which they watched a three-minute video clip featuring a Hubble photograph of the starry sky with peaceful music played through their headphones. The 36 trials (in which participants chose one of two curtains they believed an "image" was behind, on a computer screen) began immediately after the relaxation period. If they chose the correct side, the image would then be displayed, otherwise a black square would be displayed instead. After completing the 36 trials, participants filled out the questionnaire, and were then provided with a full debrief on the nature of the experiment (i.e. that the image was generated only after the participants' made their choices, as a test for precognition specifically).

\section{Results}

Although we were only interested in individual difference predictors of psi performance (as opposed to the general performance of all participants), we still report this outcome, as it was the primary outcome of Bem's (2011a) original experiment. Across all 208 participants, participants correctly identified the future position of the erotic images more frequently than the $50 \%$ hit rate expected, but it was not statistically significant: $50.9 \%, \boldsymbol{t}(207)=1.16, \boldsymbol{p}=$ 
.138. The participants' hit rate on the non-erotic images did not significantly differ from chance: $49.6 \%, \boldsymbol{t}(207)=.41, \boldsymbol{p}=.705$. The fact that the control condition (i.e. non-erotic image trials) did not differ from chance, suggests that the RNG was not biased, and we can be more confident that our results are not due to a predictable randomisation sequence (as discussed in Chapter Two).

Descriptive data for all variables analysed are provided in Table 3 below. The reliability of the scales for transliminality $(\boldsymbol{a}=.78)$ and magical ideation ( $\boldsymbol{a}$ $=.74$ ) are acceptable, as well as the two-item reliability coefficient for extraversion $(r=.61)$ (even though .80 is ideal, as lower alphas in this range are often acceptable; Giles, 2002). However, the two-item reliability coefficient for openness to experience is very low $(r=.15)$, which suggests that a large part of the variance using this measure could be due to factors beyond the actual construct (e.g. how it is worded, or how participants are feeling at the time). Nevertheless, Gosling et al. (2003) claim that the TIPI emphasises content validity considerations (due to only having two-items), and therefore, validity exceeds reliability resulting in unusually low internal consistency estimates. For this reason, they suggest using less biased (by efforts to retain content validity) reliability estimates such as test-retest reliability correlationsthere, they found an $\boldsymbol{r}=.62$ for openness to experience (in contrast to $\boldsymbol{r}=.28$ for the openness to experience two-item correlation, which is similar to what we found).

Table 3

Descriptive Statistics $(N=204)$ for Belief in ESP, Extraversion, Openness to Experience, Transliminality, Magical Ideation, and Psi Performance

\begin{tabular}{|c|c|c|c|}
\hline Measure & $\alpha$ & $\mathrm{M}$ (max score) & SD \\
\hline Belief in ESP & & $2.91 \quad(5)$ & 1.03 \\
\hline Extraversion & $.61^{*}$ & $4.12 \quad(7)$ & 1.38 \\
\hline Openness to Experience & $.15^{*}$ & $5.25 \quad(7)$ & .96 \\
\hline Transliminality & .78 & $23.83 \quad(37.3)$ & 3.90 \\
\hline Magical Ideation & .74 & $10.47 \quad(30)$ & 4.47 \\
\hline Psi Performance & & $9.16 \quad(18)$ & 2.04 \\
\hline
\end{tabular}


To avoid multiple analyses and the criticisms of Bem's (2011a) initial paper, only the three individual difference factors that were hypothesised to predict performance on the precognition task were analysed (along with the two exploratory individual differences). The correlation between belief in ESP and performance was significant $(\boldsymbol{r}=.16, \boldsymbol{p}=.02 ; 95 \%$ CI $[.02, .29])$, whereas the correlations between extraversion $(\boldsymbol{r}=.05, \boldsymbol{p}=.49 ; 95 \% \mathrm{CI}[-.09, .18)$ and openness to experience $(\boldsymbol{r}=-.04, \boldsymbol{p}=.61 ; 95 \%[-.17, .10])$ were nonsignificant. The expected range ( $r$ 's) from Study I (based on a 95\% confidence interval) are presented in Table 4 below, along with the results of this study. As can be seen, the outcome for belief in ESP and extraversion fall within the range of the confidence intervals identified in the meta-analysis in Chapter Three, but openness to experience falls outside of this range.

Table 4

Comparison of Study II Results with the Confidence Intervals Found in Study I

\begin{tabular}{llll}
\hline Individual Difference measure & $\begin{array}{l}\text { Study I (95\% } \\
\text { Confidence } \\
\text { Interval) }\end{array}$ & $\begin{array}{l}\text { Study II results } \\
(r)\end{array}$ & $\begin{array}{l}\text { Falls within the } \\
\text { expected range? } \\
(\mathrm{Y} / \mathrm{N})\end{array}$ \\
\hline Belief in ESP & $.07, .20$ & $.16^{*}$ & $\mathrm{Y}$ \\
Extraversion & $.01, .15$ & .05 & $\mathrm{Y}$ \\
Openness to Experience & $.03, .20$ & -.04 & $\mathrm{~N}$ \\
\hline
\end{tabular}

$* p \leq .05$

\subsection{Exploratory Analyses}

The relationship between performance and the two exploratory individual difference predictors, transliminality $(r=.06, p=.39)$ and magical ideation $(r=.14, p=.06)$, were also non-significant. They were therefore no better than chance at predicting performance on this forced-choice precognition experiment, although magical ideation was approaching significance.

A matrix of correlation coefficients between all predictor variables and psi performance was also computed (see Table 5 below) to observe the relationships between the different scales. The strongest significant correlations were found between belief in ESP and both magical ideation $(\boldsymbol{r}=.53, \boldsymbol{p}<$ $.001)$ and transliminality $(\boldsymbol{r}=.43, \boldsymbol{p}<.001)$, and between magical ideation 
and transliminality separately $(\boldsymbol{r}=.67, \boldsymbol{p}<.001)$. This suggests that belief in ESP, magical ideation, and transliminality are significantly related to each other, such that participants high in one scale are likely to be high in the other scales also.

Weaker but significant correlations were also found between openness to experience and both extraversion $(\boldsymbol{r}=.22, \boldsymbol{p}=.001)$ and transliminality $(\boldsymbol{r}=$ $.30, \boldsymbol{p}=.001)$, but not between extraversion and transliminality separately $(\boldsymbol{r}$ $=.10, p=.17)$. No significant correlations were observed between belief and ESP and both extraversion $(\boldsymbol{r}=.02, \boldsymbol{p}=.79)$ and openness to experience $(\boldsymbol{r}=$ $.11, p=.13)$. Similarly, magical ideation was not significantly correlated with extraversion $(\boldsymbol{r}=.02, \boldsymbol{p}=.75)$, or openness to experience $(\boldsymbol{r}=.10, \boldsymbol{p}=.17)$.

Table 5

Matrix of Pearson Correlations Between Predictor Variables and Psi Performance

\begin{tabular}{lllllll}
\hline & 1 & 2 & 3 & 4 & 5 & 6 \\
\hline 1. Belief in ESP & - & & & & & \\
2. Extraversion & .02 & - & & & \\
3. Openness to Experience & .11 & $.22^{* *}$ & - & & & \\
4. Magical Ideation & $.53^{* *}$ & .02 & .05 & - & & \\
5. Transliminality & $.43^{* *}$ & .10 & $.30^{* *}$ & $.67^{* *}$ & - & \\
6. Psi Performance & $.16^{*}$ & .05 & -.04 & .14 & .06 & - \\
\hline${ }^{*} p \leq .05, * * p \leq .01$ & & & & &
\end{tabular}

As it was suggested in Chapter Three to combine significant predictors together for additive power, a multiple regression analysis was also performed using the main three measures (belief in ESP, extraversion, openness to experience) to predict psi performance. This model did not significantly predict psi performance $\left(\boldsymbol{R}^{2}=.03, \boldsymbol{F}(3,200)=2.21, \boldsymbol{p}=.09\right)$. Consequently, only one beta-value was significant and of moderate strength (see Table 6 below). 
Table 6

Multiple Regression with Belief in ESP, Extraversion, and Openness to Experience Predicting Psi Performance

\begin{tabular}{llcc}
\hline & $\mathrm{B}$ & $\beta$ & $t$-value \\
\hline Belief in ESP & .33 & .17 & $2.38^{*}$ \\
Extraversion & .09 & .06 & $.83 \mathrm{~ns}$ \\
Openness to Experience & -.14 & -.07 & $-.92 \mathrm{~ns}$ \\
Constant & 8.57 & & \\
\hline
\end{tabular}

${ }^{*} p \leq .05$

As the table shows, belief in ESP was the only predictor of psi performance. Extraversion and openness to experience scores did not account for significant variance in psi performance when considered alongside the other two predictors. This supports the earlier finding above that belief in ESP singlehandedly predicted psi performance on this task.

\section{Discussion}

While there were a lot of additional variables that could have been explored in this experiment, the aim of this thesis is to bring structure to the field and address concerns that have encumbered parapsychology for decades (Alcock, 2003; Hyman, 1977, 1985, 1995). Therefore, we chose to conduct a straightforward replicatory experiment using Bem's (2011a) resources, so as not to reinvent the wheel while also enabling a form of pre-registration that is inherent in the replication of a previous experimental paradigm (both in the ethics application that is submitted prior to the running of the experiment, and in the use of the same software which restricts the amount of exploration one can do).

The main criticism of Bem's (2011a) experimental procedure that we sought to address in this confirmatory study was eliminating the possibility of post-hoc exploration of the data. So, for example, Yarkoni (2011) claimed that Bem's (2011a) use of different numbers of participants per study was highly suspicious, while Francis (2012) claimed that the numbers of participants were too low given the effect sizes and power of his studies. Therefore, we conducted a power analysis before conducting Study II that confirmed the numbers of 
participants that we needed to include, and then pre-specified this minimum number in the ethics application form before we began. Another issue was that Bem (2011a) presented his experimental designs as fixed prior to collection of his data, even though there is reason to think he altered experimental protocol during data collection, pooled participants arbitrarily, and presented post-hoc hypotheses as pre-planned (Alcock, 2011; Schimmack, 2012; Yarkoni, 2011). By using Bem's (2011a) software directly, we were able to take everything he did at face value and keep it all fixed, without the possibility of post-hoc changes. Lastly, Wagenmakers et al. (2011) and Alcock (2011) argue that Bem (2011a) never made it clear how many analyses he ran in total and did not account for multiple analyses. Further, Yarkoni (2011) claims that Bem's (2011a) use of one-tailed tests was unjustified. We therefore make it clear in our study that we only had three pre-specified hypotheses and two exploratory analyses, all of which were analysed using two-tailed tests (although it could be argued that one-tailed tests are also adequate, given that our hypotheses are based on his results). We leave it up to the reader to draw his or her own conclusions based on the empirical evidence.

Overall, belief in ESP was the only two-tailed predictor of psi performance (identified in forced-choice precognition experiments from Study I) that was successfully replicated in Study II; extraversion and openness to experience failed to reach statistical significance. One interpretation is that this result represents a false positive finding, which will occasionally occur. Alternatively, belief in ESP may be a true predictor of psi performance, consistent with previous meta-analyses that have found strong support in the forced-choice domain for what is commonly known as the sheep-goat effect (Lawrence, 1993; Storm \& Tressoldi, 2017). The latter possibility is strengthened by the fact that belief in ESP was the most robust predictor found in Study I $(r=.13, p<.001)$; its confidence interval is furthest away from zero compared to all other predictors analysed, and its summary statistics are based on almost twice as many independent investigators and studies than the next largest predictor in Study I. 
What is interesting with the results (i.e. no main effect, but an overall significant belief in ESP effect) is that this is consistent with an experimenter effect, or what is known as the parapsychological experimenter effect (Irwin \& Watt, 2007). In essence, many parapsychologists believe that while psi does exist, it is difficult to pinpoint or reliably replicate, as the results are influenced by the psi abilities of the actual experimenter (e.g. an experimenter may inadvertently help or inhibit participants in a parapsychological experiment, in a way that is consistent with their beliefs). Some critics argue that this is a circular claim, as one must already assume psi exists to accept it as an explanation (Alcock, 2003). On the other hand, if psi were real, it is inevitable for experimental results to be inconsistent, given that we would never have a truly controlled experiment (until we understand exactly when psi can and cannot occur). In fact, some experiments have attempted to isolate this effect (e.g. Watt \& Ramakers, 2003; Wiseman \& Schlitz, 1997), although replication attempts are not always successful (Schlitz, Wiseman, Watt, \& Radin, 2006). Here, a belief in ESP effect is not the same as an experimenter effect, but it does suggest that some people (i.e. 'sheep') can use their psi ability to perform better in an experimental laboratory task, while others can use it to perform worse (i.e. 'goats'). It is not too hard to imagine researchers also using their own psi to influence the outcome of an experiment, just as participants did in Study II (potentially). Future researchers might therefore look to belief in ESP as a filter to examine the parapsychological experimenter effect.

In this study, a moderate correlation was also found between the measures of ESP belief, magical ideation, and transliminality, which is consistent with previous findings of transliminality (and its underlying variables including magical ideation) being correlated with the sheep-goat variable (Thalbourne \& Houran, 2003; Thalbourne \& Storm, 2012). Thalbourne and Houran (2003) argue that the correlation is so high that they are practically measuring the same construct, which suggests multicollinearity (however, in our study, belief in ESP and transliminality only share $28 \%$ of their variance, so this claim is not supported). Thalbourne and Storm (2012) compared both the 
sheep-goat variable and transliminality as predictors of psi performance and found that pre-1971, transliminality had produced more significant outcomes than the sheep-goat variable, but it had reversed post-1971. Thalbourne and Storm (2012) therefore argue that best practice should be to administer both the sheep-goat variable and transliminality concurrently to see which predicts psi performance better, since transliminality contains more underlying processes and may therefore prove to be superior to the sheep-goat variable. However, based on our findings in both Study I and Study II, belief in ESP is a significant predictor of psi performance whereas transliminality is not, even though the variables are moderately correlated with each (as seen in Study II). These differences may be due to the fact that we are looking at the forcedchoice precognition domain specifically, whereas Thalbourne and Storm (2012) were looking at predictors of psi performance in a range of contexts. Nevertheless, belief in ESP seems to independently predict psi performance in the forced-choice precognition domain.

One limitation in this study is the weak inter-relationship between the two items assessing openness to experience $(r=.15)$. While Gosling et al. (2003) claim that this correlation is not a fair representation of its reliability (as it does not account for content validity considerations), it is nevertheless a consequence of being a two-item measurement. The benefits of using the TIPI as a Big-Five instrument, is that it is not time-intensive and, in Study II, allowed us to keep experimental sessions to 30 minutes (the questionnaire was already rather long and they still needed time to complete the experimental task). However, Gosling et al. (2003) did find that Extraversion fared the best across their testing criteria (convergent discriminant validity, test-retest reliability, patterns of external correlates, and convergence between self and observer reports) whereas Openness to Experience fared least well. It is possible, then, to interpret the results of Study II as being partly due to the limitations of the TIPI. In particular, the least reliable measure Openness to Experience $(\boldsymbol{r}=-.04, \boldsymbol{p}=$ .61) was the only predictor to fall outside of the confidence interval range found in Study I $(.03, .20)$, whereas the most reliable measure Extraversion ( $\boldsymbol{r}$ 
$=.05, \boldsymbol{p}=.49) \mathrm{did}$ fall within this range $(.01, .15)$-even though it was not statistically significant. Future forced-choice precognition research may therefore look to incorporate more thorough Big-Five instruments, if time allows for it (e.g. the 240-item NEO-PI-R; Costa \& McCrae, 1992).

Another limitation is the potential order effects, as the questionnaire was administered to participants after (rather than before) the experimental task. Participants may therefore have been influenced by how well they did in the experimental task, and answered the questionnaire accordingly (e.g. if they got many trials correct, they may have answered certain questions about luck more favourably). In fact, Honorton, Ferrari, and Bem (1998) concluded that extraversion predicted psi performance (in forced-choice ESP experiments) only when extraversion was measured after (but not before) the experimental procedure. However, in our study, extraversion and openness to experience was not predictive of psi performance, so order effects did not seem to have the same influence. Belief in ESP was significant, but this predictor is unlikely to have been affected by order effects, as this measure was administered prior to the experimental trials (as it was built into Bem's replication package). Overall, it would have been better to administer all of the individual difference measurements before the experimental trials, in order to avoid any ambiguity that might arise out of order effects.

While laboratory precognition experiments are useful, it cannot provide the whole story on precognition. Why? Well for one, Study II is conducted in a somewhat artificial context, with people coming into the laboratory only for course credit, doing a task they would never otherwise do, with little to no motivation to perform well. For that reason, we will put all of this to the test again in Study III (the following Chapter) by analysing these same individual differences in a real-world lottery study, in an attempt to see whether these differences are predictive in a real-world setting where people actually choose to do these 'psi' tasks on their own accord. This is based on several recommendations by other researchers who argue that psi phenomena is only useful and convincing insofar as it can predict meaningful real-world events 
that produce tangible outcomes (e.g. Franklin, Baumgart, \& Schooler, 2014). There is no place better to look than the lottery. 


\section{CHAPTER FIVE: APPLIED FINDINGS}

\section{Introduction}

The main purpose of any research, in the end, is to understand the world better. It is conventional to conduct an experiment in the laboratory-but without further testing, it is difficult to be certain of its relevance in the real world (especially since there are often many other variables that potentially negate the effects found in an experimental setting). Therefore, the results from Studies I and II are going to be extended in Study III, in an attempt to validate the findings in the real world.

It is especially important that we test psi effects in the real world. The reason is twofold: first, if it cannot be shown to exist in the real world, then it implies that, even if psi is real, its effect is too small to meaningfully impact us, and thus not worth thinking about. It follows then, that the only way to give credence to the importance of parapsychological research is to explore psi in the real world and make psi visible in some way or form. Franklin, Baumgart, and Schooler (2014) alluded to this, when they urged researchers to explore precognition "through the development of paradigms that use software in realtime to predict meaningful future outcomes before they occur" (p. 4) with the goal of showing "tangible effects applied in real-world settings" (p. 2). As an example, they suggest an experiment to predict the outcome of a roulette wheel (either landing on red or black) and actually placing a bet on it in real-time, to show psi's money-making (and thus real-world impacting) potential. For example, experimental targets (A, B) could be mapped to either "red" or "black" on a roulette wheel, such that participants' faster reaction times (on targets $\mathrm{A}$ or B) will determine whether a bet is then placed on "red" (A) or "black" (B). After the roulette wheel is spun, the colour that the ball lands on will determine which target is going to be practiced by the participants afterwards (either A or $B$ ), such that a retrocausal practice effect will lead to more successful bets than non-successful ones, as people are more likely to choose the target corresponding to the winning colour precisely because that is the target they 
practice in the future. Such an example is, in effect, a real-world application of Bem's Experiments' 8 and 9 (retrocausal practice effect).

Second, there are many counter-examples to psi in the real world, such as casinos consistently making money, that allow sceptics and even openminded individuals to dismiss laboratory psi evidence outright (as one would assume that if psi existed, people would use it to win money off the casinos at higher rates than observed). That is not to say that casinos consistently making money off slight statistical house edges does in fact prove that psi is impossible, since experiments favouring psi show only a slight deviation from chance-a deviation typically so small that it would likely not outweigh the house edge. Furthermore, if believers in ESP could influence casino outcomes positively and disbelievers negatively (as suggested from the results in Studies I and II), then casinos are likely to remain profitable given that the effects of all casino-goers are likely to cancel each other out. Nevertheless, just because it is possible for psi to exist does not necessarily make it probable, so real world parapsychological studies need to be conducted to test psi hypotheses.

To further explain how psi might be possible even with casinos flourishing - the actual house take is usually much larger than its theoretical advantage, since people rarely play optimally and often reinvest their winnings (i.e. lose some money back to the casino even after winning). So even if you could use psi to tilt the odds in your favour, it is unlikely to occur in the real world given that a casino is designed to be distracting and prevent careful thought and concentration (whereas experimental conditions are designed to be as psi-conducive as possible, e.g. quiet and relaxing with few distractions) (Irwin \& Watt, 2007). This is why experimental sessions are usually limited to 15-30 minutes (to mitigate boredom and fatigue). To win at the casino over the long run, people would need to perform consistently at an optimal level, perhaps over a period of months or even longer. And even if a few people did beat the casino, the percentage of winners would be so small that it is unlikely to put a dent in the casino's profits. So regardless of whether psi is real or not, 
casinos are not going to provide the conclusive evidence we are looking for (i.e. they are not going to go bankrupt any time soon).

To better understand real-world psi research, Milton and Wiseman (1999a) conducted a meta-analysis on all large-scale psi experiments that have tried to bridge the gap between laboratory testing and real world demonstrations of psi. They considered all psi experiments that were conducted using newspapers, radio, magazines, and television, where listeners or readers were asked to predict random targets (such as numbers or sequences), analysing over 1.5 million trials from 8 studies, and found that the overall hit rate did not significantly differ from chance (the effect was slightly negative). Given that the statistical power of all of these trials combined was large enough to detect an effect, these results suggest that either (a) psi does not exist and the effect size is an accurate depiction of the likelihood of guessing an unpredictable event, or alternatively (b) the study conditions were very different to other successful psi experiments (which may very well be the case, given that laboratory psi experiments often create an environment with no distractions, unlike the real world).

In the absence of a meta-analytic effect in these real-life psi tests, why attempt to add to these studies? Firstly, the findings may not describe the full picture, as Milton and Wiseman (1999a) do not differentiate between the different types of psi being looked at (e.g. precognition vs. clairvoyance). Secondly, all results are generalised even though there is reason to suggest that some individuals high in a certain trait (such as belief in ESP) might perform better on psi tasks than those low on that same trait (Lawrence, 1993; Storm \& Tressoldi, 2017; Zdrenka \& Wilson, 2017). Without taking into account these individual differences, potential psi effects may be masked, which may have similarly occurred in Study II as we did not find a general psi effect yet a sheepgoat effect did emerge. Milton and Wiseman's (1999a) meta-analysis may therefore be useful as a baseline of what we might expect in the real world, but there is still need for further exploration-especially in the precognition forced- 
choice domain as the results of Study I and II suggest. Study III will therefore look at individual difference predictors in a real world lottery setting.

A few studies have followed a similar line of research (e.g. looking at the predictors of lottery success in the real world). Smith, Wiseman, and Harris (1997) looked at perceived luckiness, confidence of a win, lottery playing behaviour, and actual success in the UK National Lottery, and elicited responses using a postal questionnaire. They chose the lottery for the following reasons: (1) the large amount of prize money is more of an incentive than the rewards provided for participation in a typical laboratory experiment, (2) some people claim—anecdotally—that they are "lucky" or have had a lot of luck when predicting the lottery, (3) previous research by Broderick (1992) and Zilberman (1995) suggest that lottery data may evidence psi effects, (4) the lottery is highly randomised and has security to prevent cheating (5) the national lottery is very popular-the UK one in particular has a $65 \%$ play rate, so participants will be familiar with it.

Smith and colleagues (1997) found that participants who thought they were lucky expected to win significantly more than unlucky participants, however, none of the luckiness measures predicted actual lottery success. Overall, this suggests that there is little difference between lucky and unlucky participants (in terms of perceived luckiness) in games of chance in the real world. However, given the findings of Study I and II, there is reason to believe that in a real world instance of forced-choice guessing of a future event, there may be other individual differences that predict overall performance (not just limited to that which has been measured in mass media tests, e.g. perceived luckiness). A lottery study is a perfect extension of Study I and II, as it assesses precognition (guessing an unpredictable future event) while also being forcedchoice (participants must pick from a limited number of options, i.e. the numbers 1-40). Watt and Nagtegaal (2000) conducted a similar experiment with participants predicting the UK National Lottery-and although they did not find lucky participants to do any better than unlucky participants, they did find that participants who specifically believed that their luck could affect 
their lottery performance successfully guessed more correct numbers than those who did not believe that their luck could affect their lottery performance.

Researchers have also investigated the lottery more generally. For example, Broderick (1992) analysed lottery data from the Australian National Lottery, trying to determine whether there were more successful picks overall than one would expect by chance alone. However, the difficulty with lottery data-or basically any psi experiment where only a single target set is used for all participants-is that people's biases or preferences for certain numbers make it difficult to determine whether any above-chance results in the data might be attributable to these biases (rather than psi). This was demonstrated strongly in a study looking at response biases by Lund (1939), where 596 participants each guessed a random sequence of five Zener card symbols (wavy lines, cross, circle, star, square) and found that the star was chosen $32 \%$ of the time (cf. the $20 \%$ expected by chance if all symbols have an equal chance of being chosen)almost double the frequency of the next most popular symbol (the square).

This led Greville (1944) to develop a method attempting to correct for such biases known as the Greville correction. For example, Nash (1964) applied the Greville correction to reduce a 30-fold overestimation of an audience guessing a number between 1-1,000,000 on a nationally broadcast television show, since participants had a strong preference for numbers under 100,000 ( $80 \%$ of the participants opted for a number under 100,000 even though there is only a $10 \%$ probability of the number being less than six digits if the number was randomly chosen). To exemplify what this might look like in a lottery study, imagine the possibility that the winning lottery numbers (a shared target set) for one week includes the numbers seven and three. If so, there will be a disproportionate number of hits that week (as seven and three are the most popular numbers that people pick), but this will not be due to psi-rather, it will be due to preferences matching the 'chance' occurrence of a particular shared target. Not all biases will be fixed either; people also pick numbers based on external (and constantly changing) factors such as the current date, or previous draws' winning numbers (Halpern \& Devereaux, 1989). This is why 
Milton and Wiseman (1999a), in their meta-analysis of large-scale psi experiments, excluded studies that only used a single target set and did not adjust for such biases using the Greville correction.

Similar attempts have been made to account for such guessing habits. For example, Broderick (1992)—when analysing the lottery data-was aware of the need to factor in these biases when he stated that, "[i]n a given Lotto draw, [one must ask if] the winning numbers attract a vote significantly higher or lower than their usual vote in weeks when they were not winners" (p. 207). In other words, he looked for the base rate of how frequently a number was picked, before determining whether the hit rate of a winning number differed from the base rate expectation of that same number on non-winning draws. Otherwise, erroneous conclusions can be found if "experimenters assume independence of calls and use the theoretical variance rather than the observed variance of the distribution of responses [as the] difference between theoretical and observed variance can be extreme" (Milton \& Wiseman, 1999a, p. 237). However, just comparing the proportion of winning numbers picked with the proportion of these same numbers picked on non-winning dates is not entirely flawless, as there may still be external factors influencing people's preferences at any given time that may skew such averages (e.g. Christmas time may change people's preferences for a single draw on that week, or winning numbers for one week may influence people's predictions for the subsequent week).

Nevertheless, Broderick (1992) analysed 23 consecutive draws of lotto from 9 February to 24 April 1991, which contained more than 848 million entries. Using the approach outlined above, while also exploring the data with multiple linear regression (which has been criticised because the data cannot be linear, so even if some data fit the curve, it must only be temporary and cannot provide evidence of any systematic patterns), he was unable to find evidence that psi was operating. However, Broderick (1992) claims that if psi were operating, the effect would be so small that once too many people's choices were included in the results, any psi effect would become masked. 
Alternatively, a 'sheep' effect may be balanced out by a 'goat' effect, with a net outcome indistinguishable from chance (as mentioned previously). This is a common issue when results of a population are generalised-and is the main reason why this thesis focuses so heavily on individual differences in psi performance, as psi abilities may not be equally distributed amongst the population. Furthermore, Broderick's (1992) data used all lottery tickets in a draw, which would include both self-selected numbers and randomly selected numbers-the latter of which is unlikely to contain a psi effect and may merely dilute any psi effect in the overall data.

Broderick (1992) then decided to look at the biggest positive residuals in the data and compare them with winning numbers, on the basis that a psi effect would be too small to be found in general but may emerge in the extreme ends of the data. He therefore analysed the top one percent of positive residuals ( 9 out of 900 numbers) and found that the majority of these deviations predicted actual winning numbers (7 out of 9 ) whereas only 1.38 out of every 9 might be expected for any random sample chosen. Of course, as per the garden of forking paths (see Chapter Four), any selected group of numbers can, post-hoc, be rationalised as being significant even though this exact outcome is just as likely as any other (if not pre-specified). Broderick (1992) also looked at the top and bottom ten percent of residuals in further analyses (and split up the dataset into midweek and Saturday draws) and found less strong correlations, so a lot of exploration seems to be going on. It is inevitable that some of these findings would be significant, due to chance alone. Similarly, PEAR's (Princeton Engineering Anomalies Research Lab) Roger Nelson contends that Broderick's cut-off criteria for the largest residuals were "arbitrary (not pre-specified) and might have been selected after trying other criteria" (Broderick, 1992, p. 223). Broderick (1992) argues that the only three steps he took were: (1) excluding draws with atypical numbers of players (2) applying linear regression once he realised the baseline was being distorted by unforeseen trends. Then when that was not significant, (3) looked at the largest positive residuals to see if they were significantly unusual. Nevertheless, his findings are only valuable insofar 
as it can predict future psi effects rather than showing previous patterns (as patterns can be found in any dataset if one looks hard enough), so overall Broderick's (1992) analysis is not convincing.

The final lottery study example is Zilberman's (1995) data analysis of lottery results in both France and the former Soviet Union. Although the lottery is not an experiment in any scientific sense (since none of the variables are manipulated and the setting is not controlled), it is effectively still "a large-scale parapsychological experiment" where participants "are trying to predict results obtained from a Random Number Generator" with millions of data points collected (Zilberman, 1995 p. 150). Again, lotto draws are published independently in newspapers, which means that researchers cannot interfere with the results of the experiment (some have appeared to, such as Derren Brown on a live TV special—but this was likely only due to camera trickery; Revoir, 2009). But of even greater importance is that millions of individuals demand fair play, so the organisers have a huge responsibility and incentive to make sure that they use truly randomised methods. However, rather than looking at individual differences, Zilberman (1995) wanted to see if there was a correlation between lottery success and geomagnetic activity (daily average indices from the journal Solar-Geophysical Data; e.g. Chinnery, 1988) at the time.

Although Zilberman (1995) did find a relationship between geomagnetic activity and lottery success in Soviet draws (and one of two French draws), multiple analyses seem to have occurred, as lottery success was determined arbitrarily (the cut-off for lottery success was any ticket with exactly 3 winning numbers, so tickets with 4, 5, or 6 winning numbers were excluded) and many different ways of looking at geomagnetic activity were considered (e.g. comparing geomagnetic data from years in advance and comparing it with the current data) along with far-fetched post-hoc explanations of the data (e.g. explaining how natural disasters occur during data spikes). Therefore, it does not give us much useful information about the lottery. 
Overall, lottery studies have offered minimal evidence of an effect, other than potential hints using sophisticated analyses in some studies. However, it is still important to conduct further studies using the lottery, as none of these past lottery studies have incorporated the individual differences that are most predictive of psi performance in the forced-choice precognition domain (as seen by the meta-analysis in Chapter Three). Therefore, the following studiesStudies III(A) and (B) —will continue to examine these individual differences in a lottery setting.

\section{Rationale for Study III(A)}

New Zealand is a small island nation of 4.6 million people with its own government-run lottery system ('Lotto New Zealand', n.d.). It is therefore informative to know about the population at large before conducting Study III(B)—individual difference predictors of lottery success in the New Zealand lottery.

Study III(A) will examine lottery behaviours in New Zealand and a subset of previous predictors of psi success. Study III(A) and (B) will complement each other, as Study III(A) is exploratory and provides us background information on New Zealand's lottery and participants, whereas Study III(B) is confirmatory and can validate the findings of both Study III(A), and the previous Studies in Chapters Three and Four.

The aim of Study III(A) is to better understand the New Zealand lottery playing population and investigate a subset of potential predictors of lottery success.

\section{Method}

\subsection{Participants}

Readers of a national weekly newspaper (the Sunday Star Times) were invited to participate in a "Believe it or not" survey measuring the beliefs, attitudes, and superstitions of New Zealanders. Overall, 5861 participants filled 
out the survey, the majority of which were female (61\%) and identified as New Zealand European/Pākehā (81\%). Other participant' ethnicities were Māori (4\%), Other European (6\%), and Chinese (1\%). The median age was 41, with ages ranging from 14 to 71 . Though broadly representative of the adult population, the sample were also more educated and affluent than average; $35 \%$ had completed a tertiary qualification and another $20 \%$ had completed at least one year of a tertiary programme, while median (before tax) personal income for the sample was in the "\$40,001-\$60,000 a year" category, with $15 \%$ of the sample earning over $\$ 100,000$ per year. Therefore, this sample cannot be considered fully representative of the New Zealand population. Nevertheless, it did reach a large number of New Zealanders, with participants spread across New Zealand; 29\% of participants were from Auckland, 23\% from Wellington, $12 \%$ from Christchurch, and the rest were from smaller towns/areas.

\subsection{Materials}

Paranormal beliefs were assessed using the Paranormal Beliefs Scale (PBS; Tobacyk, 2004), comprising the following seven subscales: traditional religious belief, spiritualism, witchcraft, precognition, psi, extraordinary lifeforms, and superstition. Participants' answered a total of 26 statementsconcerning the existence of paranormal phenomena-on a 7-point Likert scale ranging from strongly agree to strongly disagree. The scale has typically

provided satisfactory reliability and been used in a variety of nations and samples (e.g. Aarnio \& Lindeman, 2007; Peltzer, 2003; Tobacyk \& PirttiläBackman, 1992).

Perceived luckiness was assessed via a single item taken from Chotai and Wiseman's (2005) self-reported measure of luckiness: "In general, I am a lucky person: that is, I tend to experience lucky breaks and be in the right place at the right time," and is answered on a 5-point Likert scale ranging from strongly agree to strongly disagree. 
Lottery behaviour was investigated using three different questions. For the actual lottery predictions, participants were asked to "[i]magine that you're picking 6 numbers for regular Lotto... [and then] Select six numbers from the options below," with checkboxes ranging from 1-40, which were then compared with that week's lottery draw' winning numbers. For the frequency of lotto purchasing, participants were asked: "How frequently do you buy a lotto ticket?" and answered using a 7-point scale ranging from daily to never. Lastly, to understand whether people choose their own lotto numbers, participants were asked the following question: "If you do buy Lotto tickets, do you regularly use the same numbers?" and answered on a 3-point scale ranging from always to never.

Participants were also given a battery of questions about anomie, trust, happiness, religiosity/spirituality, participant' demographics, conspiracies, urban myths, superstitions, religious orthodoxy and fundamentalism, alternative medicine, contemporary scientific beliefs, and feelings about social groups.

\subsection{Procedure}

Following the promotion of an internet survey (examining the beliefs of New Zealanders) in the Sunday Star Times, one of New Zealand's largest newspapers, data were collected from participants during a two week period.

\section{Results}

The data are analysed in two sections. The first presents a characterisation of the paranormal beliefs and lottery behaviour, of this large sample of New Zealanders. In the second section I present analyses of lottery success, and the extent (if any) to which perceived luckiness, paranormal belief, lottery playing frequency, and number selection practices, predict success.

The majority of the sample evidenced a score indicating that they did not believe in precognition $(72.3 \%$ of the sample scored below the middle score of 
4 out of 7 on the overall belief in precognition subscale). The sample mean was $2.77(S D=1.66)$, with a mode of $1(27.8 \%$ of the sample scored the lowest possible score of 1 out of 7 on the subscale). Table 7 shows a summary of the individual items scored in the precognition subscale.

Table 7

Summary and Descriptives of Individual Items on the Precognition Subscale

\begin{tabular}{lllllll}
\hline Individual Item & $\begin{array}{l}\text { Belief \% } \\
(5-7)\end{array}$ & $\begin{array}{l}\text { Non- } \\
\text { belief \% } \\
(1-3)\end{array}$ & $\begin{array}{l}\text { Average } \\
\text { Score (out } \\
\text { of 7) }\end{array}$ & $\begin{array}{l}\text { Average } \\
\text { Score } \\
\text { (Male) }\end{array}$ & $\begin{array}{l}\text { Average } \\
\text { Score } \\
\text { (Female) }\end{array}$ & $\begin{array}{l}\text { Sex } \\
\text { Difference } \\
\text { Significance }\end{array}$ \\
\hline $\begin{array}{l}\text { 'Astrology is a way } \\
\text { to accurately predict } \\
\text { the future' }\end{array}$ & 14.2 & 76.5 & 2.24 & 1.72 & 2.72 & $\begin{array}{l}t(4433.75) \\
=21.15^{* *}\end{array}$ \\
$\begin{array}{l}\text { 'The horoscope } \\
\text { accurately tells a } \\
\text { person's future' }\end{array}$ & 9.3 & 82.8 & 1.98 & 1.57 & 2.37 & $\begin{array}{l}t(4392.79) \\
=19.28^{* *}\end{array}$ \\
$\begin{array}{l}\text { 'Some psychics can } \\
\text { accurately predict the } \\
\text { future' }\end{array}$ & 32.1 & 58.1 & 3.18 & 2.43 & 3.94 & $t(4627.22)$ \\
'Some people have \\
$\begin{array}{l}\text { an unexplained } \\
\text { ability to predict the } \\
\text { future' }\end{array}$ & 43.6 & 47.0 & 3.71 & 2.96 & 4.48 & $t(4663.91)$ \\
\hline$* * p \leq .001$ & & & & & & $=24.14^{* *}$ \\
\hline
\end{tabular}

Overall, $23.5 \%$ of the sample believe in precognition, which is more than those who indicated belief in psi, extraordinary lifeforms, or superstitious belief, but less than the percentage of participants who believe in witchcraft, spiritualism, and traditional religious belief (see Table 8 below, ordered from highest belief percentage to lowest-belief in precognition is bolded). The comparison table shows that New Zealanders are not indifferent about all paranormal concepts equally, but believe in some ideas more than others (and although these numbers are slightly lower in New Zealand, they are still fairly similar across the world; Lyons, 2005; Reader's Digest Magazine, 2006; Shermer, n.d.). 
Table 8

Proportion of Sample with Paranormal Beliefs

\begin{tabular}{lcc}
\hline PBS Subscales & Belief \% (4.25-7) & Non-belief \% (1-3.75) \\
\hline Traditional Religious Belief & 37.6 & 58.0 \\
Spiritualism Belief & 29.0 & 67.0 \\
Belief in Witchcraft & 27.6 & 68.2 \\
Belief in Precognition & $\mathbf{2 3 . 5}$ & $\mathbf{7 2 . 3}$ \\
Belief in Psi & 22.1 & 73.4 \\
Extraordinary Lifeforms & 21.5 & 71.4 \\
Superstitious Belief & 5.6 & 92.4 \\
\hline Overall PBS Score & $\mathbf{2 1 . 6}$ & $\mathbf{7 7 . 4}$ \\
\hline
\end{tabular}

$N$ 's between 5082 and 5091

There was a lot of variation in participants' lottery number picks, with the three most popular numbers (seven, 1147 participants or 20.2\%; three, 978 participants or 17.2\%; four, 941 participants or 16.6\%) being picked by over twice as many participants as the three least popular numbers (thirty-four, 349 participants, 6.1\%; thirty-five, 389 participants, 6.8\%; thirty-nine, 404 participants, 7.1\%). This is a prime example of preference biases, as the lotto number picks are not equally distributed. In fact, there is a clear downward linear trend of number picks, such that people are more likely to pick smaller numbers than higher ones (see Figure 31).

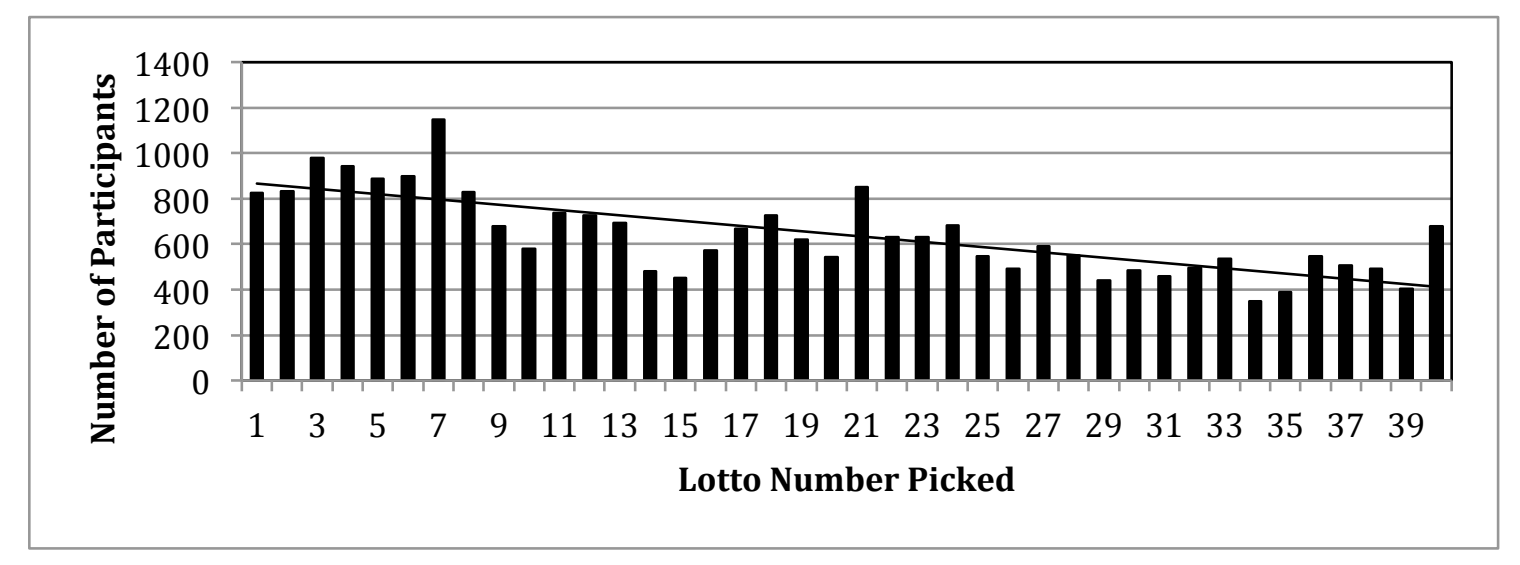

Figure 31. Bar graph showing the distribution of participants' lotto number picks. 
The majority of the participants (41.3\%) picked one correct number out of six, followed by participants picking two correct numbers (31.1\%), and then zero correct numbers (20.9\%). Overall, no participants got all six numbers correct, and only one participant got five out of six numbers correct. See the results summary in Figure 32 below to see how many winning numbers participants chose, along with the Mean Chance Expectation (MCE) for the number of winning numbers next to each column. Average outcomes were not calculated due to the non-independence calls (as seen by Figure 31$)^{6}$.

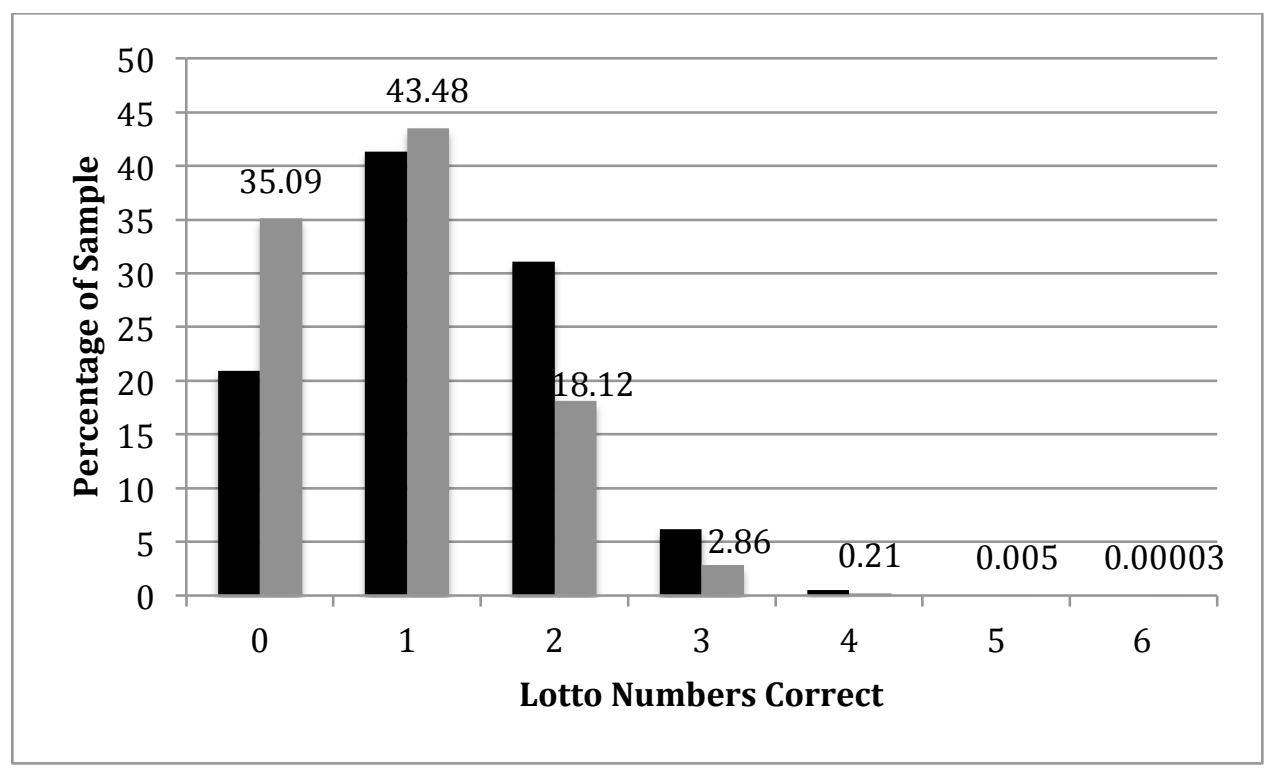

Figure 32. Bar graph showing the distribution of participants' correctly predicted lottery numbers (black columns) compared with MCE (grey columns-percentage values on top of each grey column are MCE values).

Frequency of lotto purchasing was more spread out (see Figure 33 below), with most participants having never purchased lotto tickets before (28\%), although many participants purchase tickets on a weekly (19\%), bimonthly (21\%), or yearly (19\%) basis. Monthly (7\%) and fortnightly (6\%) ticket purchasing was less common, and only a small amount of participants

\footnotetext{
${ }^{6}$ However, a one-sample t-test was calculated between the mean number of correct predictions (1.24) vs. the expected value $(0.9)$, resulting in a significant difference: $t(4101)=25.07, p<.001$.
} 
bought tickets daily (0.1\%). There are no significant sex differences in terms of lotto purchasing frequency: $t(4680.70)=-1.10, p=.27$.

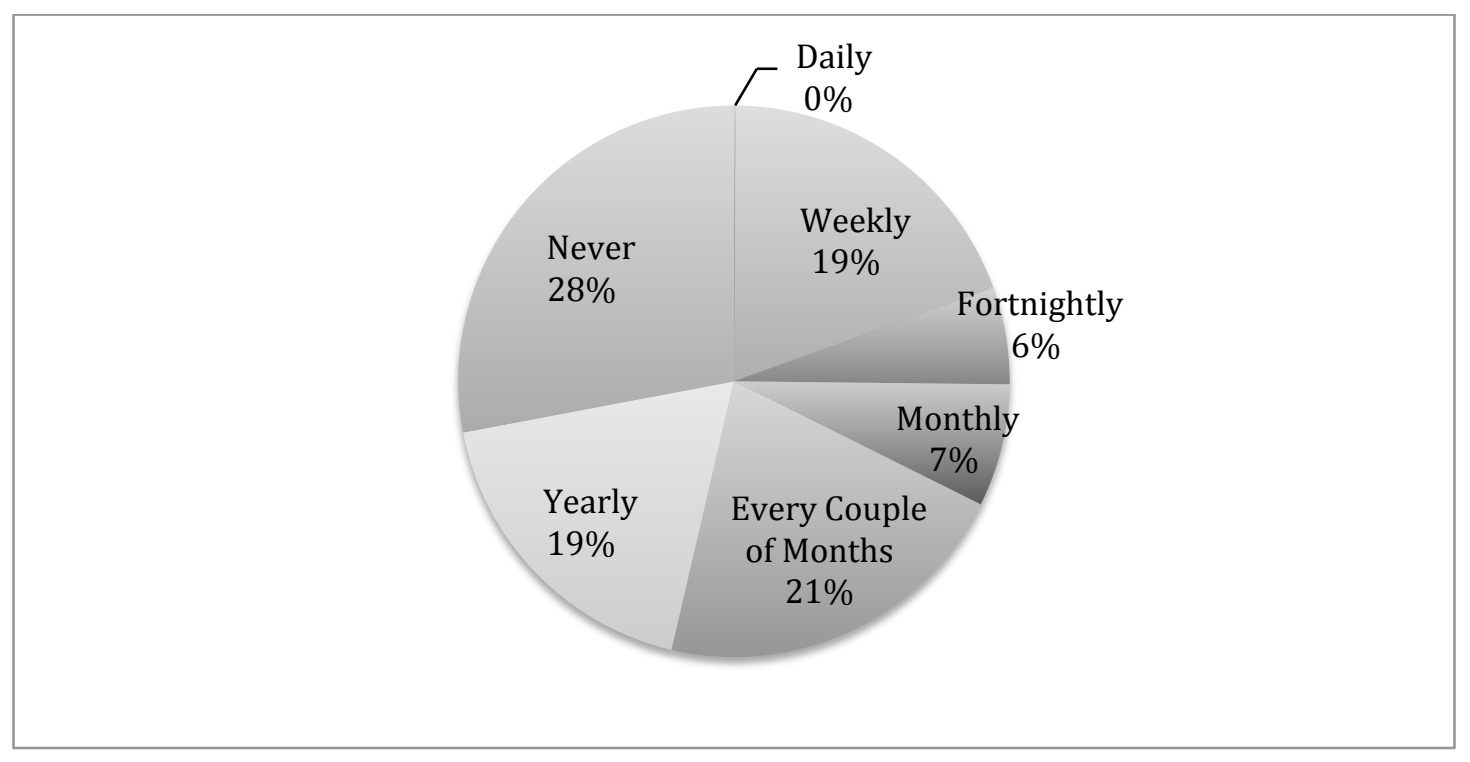

Figure 33. Pie chart showing the frequency of participants' lottery ticket purchases.

In terms of whether participants choose the same numbers each week, only $8 \%$ of participants indicated that they always select the same numbers, while $17 \%$ report that they sometimes do. The overwhelming majority never stick to a single set of six numbers for multiple lotto draws (75\%). Furthermore, participants are more likely to be frequent lotto players if they choose the same numbers each week $(\boldsymbol{r}=.38, \boldsymbol{p}<.001)$.

We also looked at lottery behaviours and whether they predicted lotto success (defined as choosing lotto numbers that were then drawn in the NZ Lottery that same week) and found no significant relationships. Lotto playing frequency was not significantly correlated with lotto performance $(r=.02, p=$ .13), as was the correlation between people who regularly pick their own numbers and lotto performance $(\boldsymbol{r}=-.002, \boldsymbol{p}=.91)$. 
Individual differences predicting lottery success were similarly examined by looking at the correlations of paranormal belief $(\boldsymbol{r}=.01, \boldsymbol{p}=.48)^{7}$ and perceived luckiness $(\boldsymbol{r}=-.02, \boldsymbol{p}=.31)$. Both correlations were non-significant and did not significantly predict lottery performance. See Table 9 below for the inter-correlations between all predictor variables and lottery success.

Table 9

Correlation Matrix between Lottery Success, Lotto Playing Frequency, Same Numbers Each Week, PBS Score, and Perceived Luckiness

\begin{tabular}{|c|c|c|c|c|c|}
\hline & LS & LPF & SNEW & PB & $\mathrm{PL}$ \\
\hline Lottery Success (LS) & - & & & & \\
\hline Lotto Playing Frequency (LPF) & -.02 & - & & & \\
\hline Same Numbers Each Week (SNEW) & .002 & $.38 * * *$ & - & & \\
\hline Paranormal Belief (PB) & .01 & $-.19 * * *$ & $-.15^{* * *}$ & - & \\
\hline Perceived Luckiness (PL) & -.02 & $-.04 * *$ & $-.04 * *$ & $.22 * * *$ & - \\
\hline
\end{tabular}

${ }^{* *} p \leq .01,{ }^{* * *} p \leq .001$

As discussed in Chapter Three, research typically looks only at single predictors for success in a psi task, so it was suggested to use multiple predictors in the same studies to look at them more holistically (rather than just separately). Therefore, to determine whether all these factors might combine to better predict lotto performance, we performed a multiple regression analysis using all four measures (paranormal belief, perceived luckiness, lotto playing frequency, lotto pick same every week) to predict psi performance. This model did not significantly predict psi performance $\left(R^{2}=.04, F(4,3716)=1.16, p=\right.$ .33). Furthermore, none of the beta-values were individually significant (see Table 10 below).

Table 10

Multiple Regression Predicting Lottery Success

\begin{tabular}{lllc}
\hline & $\mathrm{B}$ & $\beta$ & $t$-value \\
\hline Paranormal belief & .01 & .01 & .60 \\
Perceived luckiness & -.01 & -.01 & -.82 \\
Lotto playing frequency & .02 & .03 & 1.87 \\
Lotto pick same & -.02 & -.01 & .75 \\
Constant & 1.28 & & \\
\hline
\end{tabular}

All $t$ 's are not significant at $p \leq .05$.

\footnotetext{
${ }^{7}$ The precognition subscale of the PBS was also analysed separately, to see whether precognition belief could predict lottery success better than paranormal belief more generally (since the task itself is precognitive). However, it did no better than chance, $r=-.006, p=.70$.
} 
We also conducted moderated regression analyses using the interaction variables (1) perceived luckiness and paranormal belief (2) lotto playing frequency and paranormal belief (3) lotto numbers same and paranormal belief, to see whether these interactions provided additive and independent effects on lottery success. However, none of the new models significantly predicted lottery success: (1) perceived luckiness and paranormal belief, $\boldsymbol{F}(3$, $4101)=.73, \boldsymbol{p}=.54 ; \Delta \boldsymbol{R}^{2}<.001$, (2) lotto playing frequency and paranormal belief, $\boldsymbol{F}(3,4091)=.84, \boldsymbol{p}=.47 ; \Delta \boldsymbol{R}^{2}<.001$, (3) lotto numbers same and paranormal belief, $\boldsymbol{F}(3,3728)=.57, \boldsymbol{p}=.63 ; \Delta \boldsymbol{R}^{2}<.001$.

Nevertheless, a simple slope analysis was computed and graphed for all three moderated regression analyses. Figure 34 shows the moderating effect of perceived luckiness on paranormal belief predicting lottery success. From this graph, we can see that at high levels of perceived luckiness, lottery performance will increase as paranormal belief increases, while the opposite will occur at low levels of perceived luckiness (i.e. as paranormal belief increases, lottery performance will decrease). At medium levels of perceived luckiness, lottery performance will remain rather constant regardless of whether paranormal belief increases or not. 


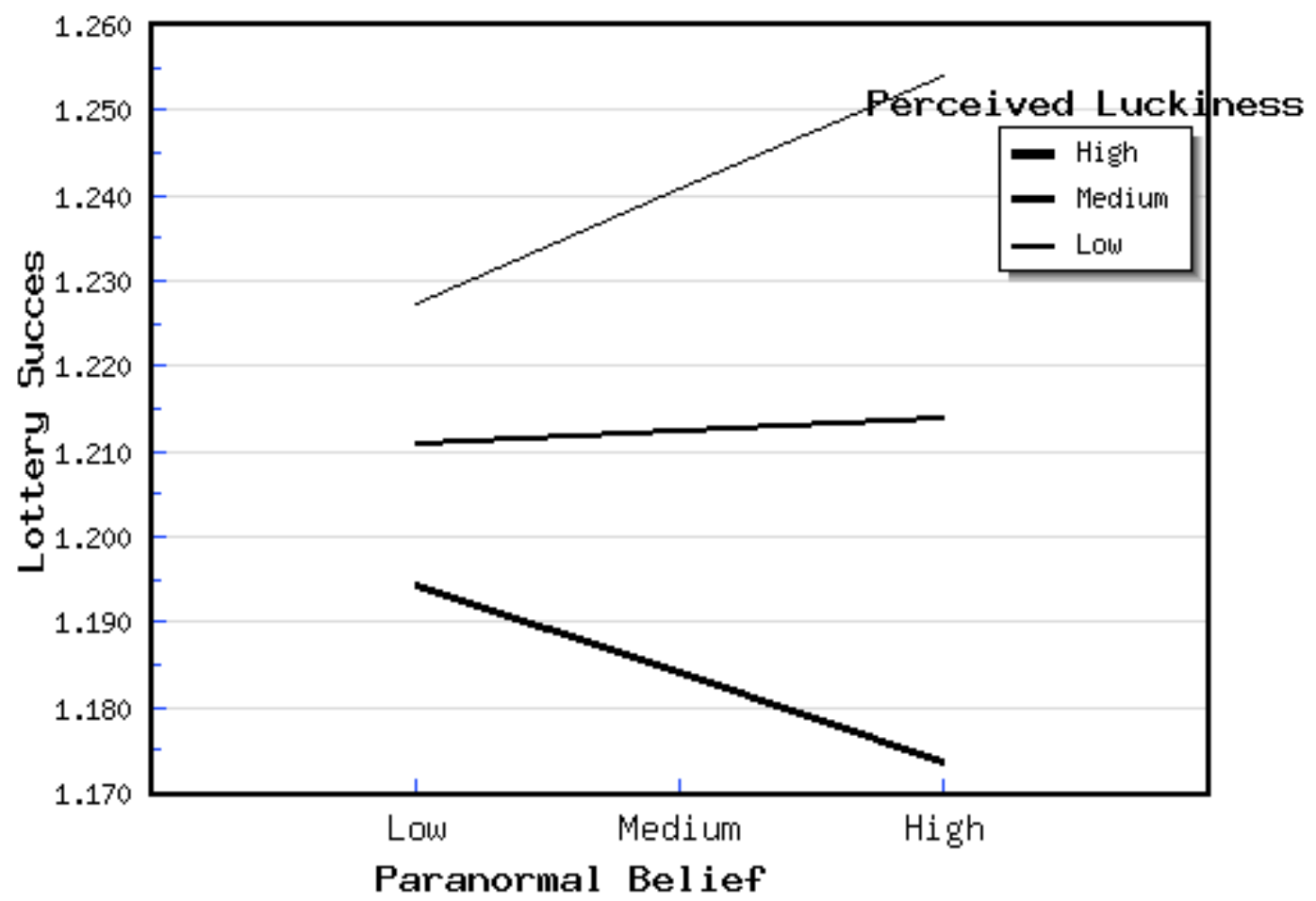

Figure 34. Simple slopes plot for perceived luckiness moderating the relationship between paranormal belief and lottery success.

The following Figure 35 shows the moderating effect of lotto playing frequency on paranormal belief predicting lottery success. From this graph, we can see that for all levels of lotto playing frequency, lottery performance will increase as paranormal belief increases. However, there also appears to be a relationship between lotto playing frequency and lottery performance, such that greater lotto playing frequency (i.e. low levels in the graph, as low numbers in this measure indicated greater frequency of play) is related to a higher base rate level of lottery success. 


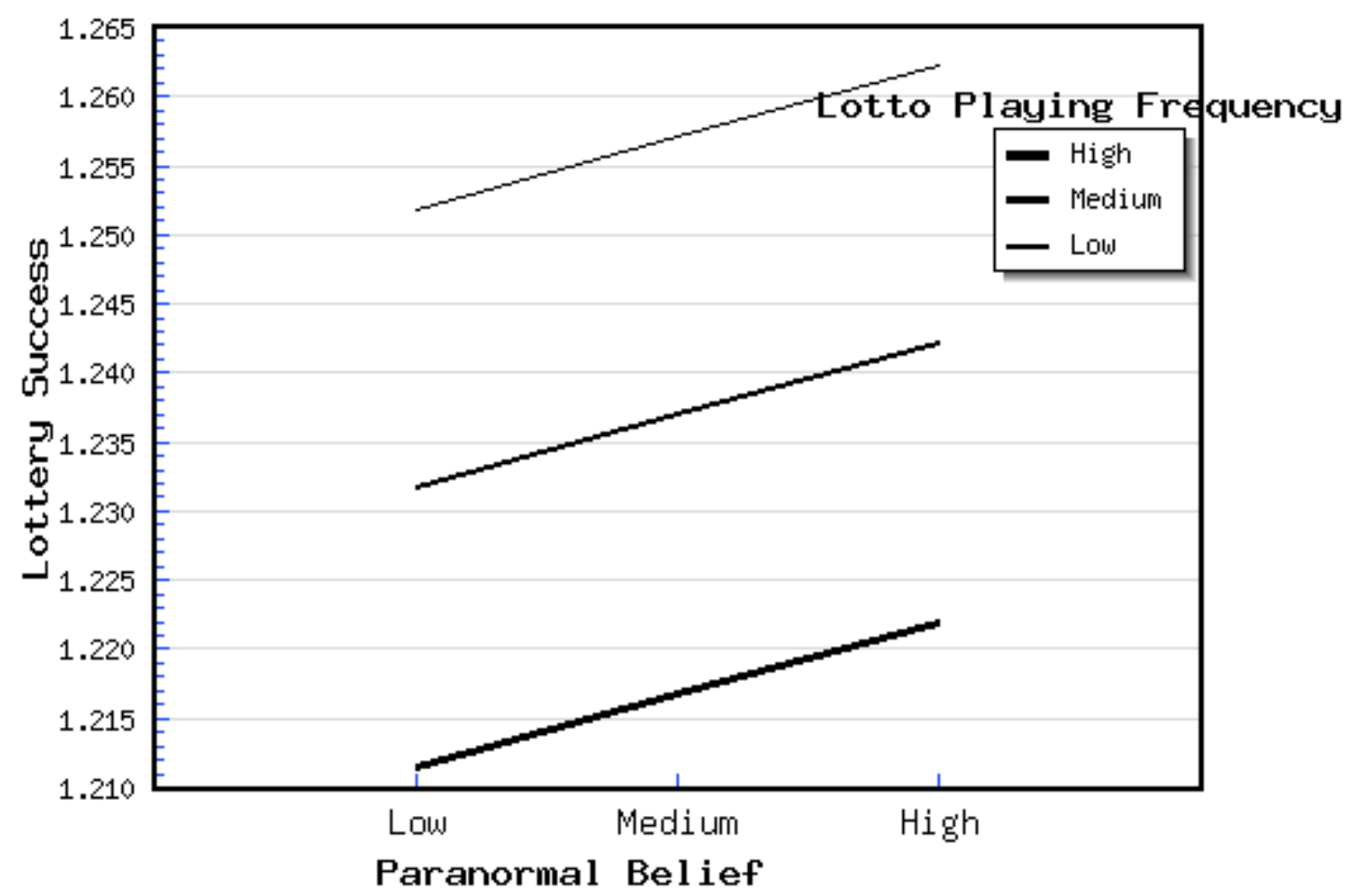

Figure 35. Simple slopes plot for lotto playing frequency moderating the relationship between paranormal belief and lottery success.

Finally, Figure 36 shows the moderating effect of picking the same lotto numbers on paranormal belief predicting lottery success. From this graph, we can see that when people were not picking the same numbers (i.e. high levels in the graph, as high numbers in this measure indicated lack of same number picks) lottery success will greatly increase as paranormal belief increases. And to a lesser extent, at medium levels of same number picks, lottery success will moderately increase as paranormal belief increases. In contrast, when people always pick the same numbers (i.e. low levels in this graph), lottery performance will remain rather constant regardless of whether paranormal belief increases or not. 


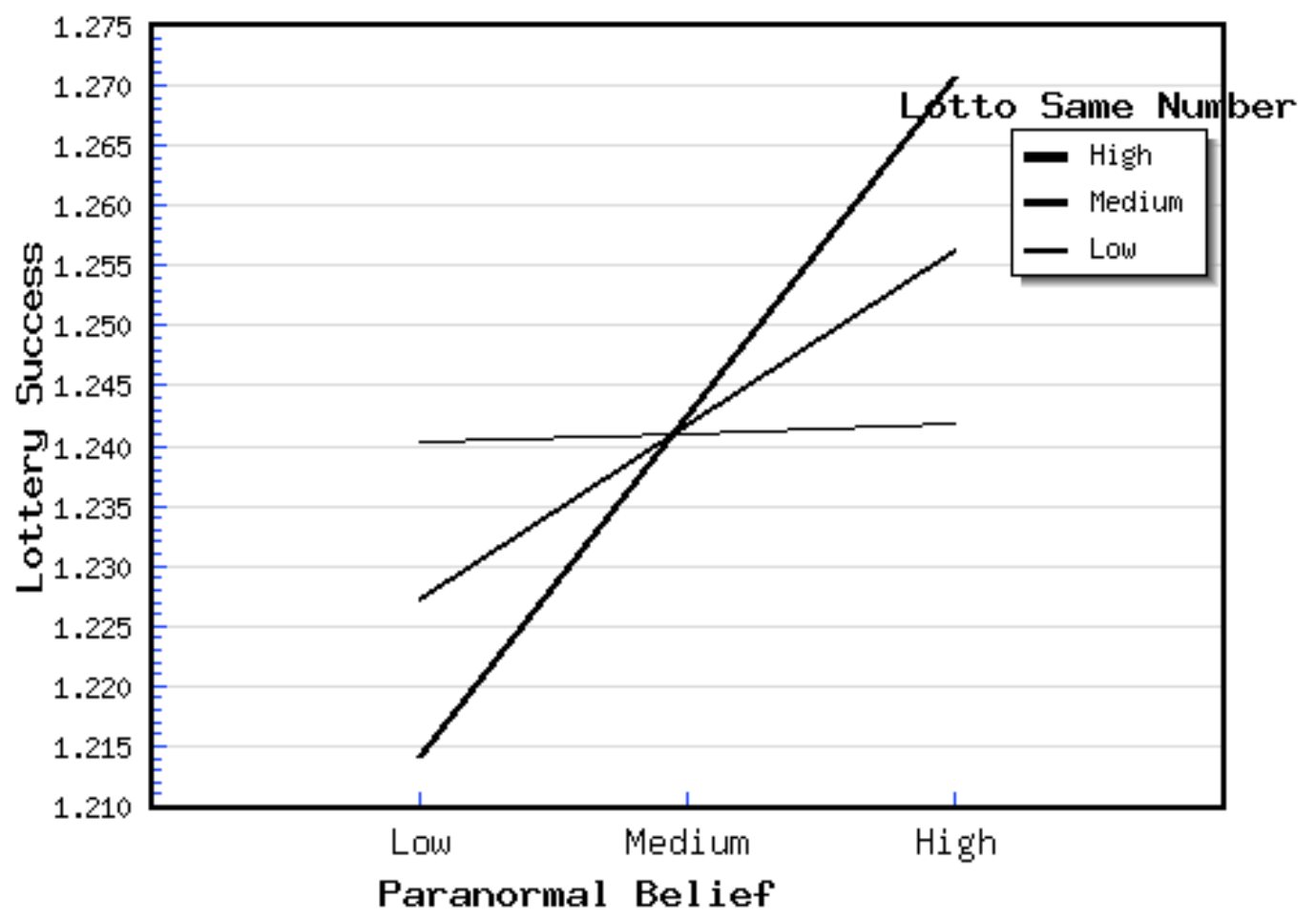

Figure 36. Simple slopes plot for same lotto numbers moderating the relationship between paranormal belief and lottery success.

However, it should be re-iterated that all of these interactions are nonsignificant and do not account for much variance (even though they may be in the expected direction).

\section{Limitations and Rationale for Study III(B)}

Study III(A) was intended as a first-step in this thesis towards evaluating the relationship between a small number of individual differences and performance in a 'real-world' psi task-the lottery. I present this study as exploratory, as the dataset was analysed after the data had been collected (and for purposes other than the research questions posed in this thesis). Nevertheless, we were interested in understanding the prevalence of precognition belief in the New Zealand population, as parapsychology stems 
from people's real world experiences (and beliefs) of psi phenomena and the aim of this thesis is to understand more about precognition in general. Overall, this sample has a low to moderate level of belief in precognition (23.5\% of the sample), and provided some indication of the nature of lotteries behaviour.

Lottery behaviour (e.g. frequency and whether or not people select their own numbers) was unrelated to lottery success. However, we did find a downward trend of lottery number preferences, which is consistent with other lottery data sets (e.g. Dutch Lotto; Wang, van Loon, van den Assem, \& van Dolder, 2016). Furthermore, seven was the most popular number chosen in our sample, which is consistent with many other experimental studies that have found seven to be participants' most preferred number choice (e.g. Silver et al., 1988; Simon, 1971). In terms of lottery purchasing frequency, we also found rates similar to a US student sample (B. A. Browne \& Brown, 1994), although we did have more regular lotto players than they did ( $25 \%$ in our sample vs. their $2 \%$ ) which may be due to students having a lower income than the general population (in which our sample came from).

We were also interested in whether paranormal belief and perceived luckiness could predict performance in New Zealand's Saturday lotto draw, since positive effect sizes were found in Zdrenka and Wilson's (2017: see Chapter Three) meta-analysis of individual difference correlates of forcedchoice precognition experiments (belief in ESP, $r=.13$; perceived luckiness, $\boldsymbol{r}$ $=.08$ ). However, we did not find such relationships in this study, so it is possible that there was no psi effect and therefore, no significant relationships between the predictors and lottery success. Furthermore, this study contained a broadly representative sample of adult New Zealanders and was adequately powered to detect any effect $(N=5861)$.

Nevertheless, there are several limitations of Study III(A) that suggest the need for a follow-up study. The first is that, because these data were collected as part of a separate research project, not all relevant predictor variables from Study I were included in the survey of Study III(A) and it was 
therefore not a comprehensive extension of Study I. Secondly, the outcome variable of lotto performance was based on a general hypothetical lotto prediction rather than a specific prediction of that week's numbers ('Imagine that you're picking six numbers for regular Lotto' rather than 'Pick the six numbers you think will appear in this Saturday's lotto draw'). Thirdly, given that there are only six winning numbers in one draw, slight differences between precognitive skill levels (assuming psi exists) may not emerge in the results of a single draw. There is a big difference in the likelihood of getting two out of six correct numbers compared to three out of six (similarly with $3 / 6$ compared to 4/6 etc.), such that the small effect sizes shown in Study I will unlikely outweigh the noise (or luck) of a single draw.

Furthermore, most of the lottery studies that have been conducted in the field are both (a) retrospective (which describe patterns in past data but do not necessarily predict future outcomes), and (b) use only a single lotto draw (which does not mitigate the potential non-independence of calls on a single shared target).

For these reasons, a pre-registered confirmatory study with all significant predictors found in Study I was designed as part of Study III(B). The confirmatory results of Study III(B) will therefore carry more weight than a non-registered study, since exploratory analyses cannot be framed as preplanned hypotheses. Furthermore, Study III(B) follows participants over an eight-week period asking them for their weekly lotto predictions (for each individual Saturday draw). This limits some of the effects that people's preferences to certain numbers might have on the prediction of a single shared target set (i.e. a single lottery draw). These factors, combined with the findings in Study I and II, allow us to better evaluate individual difference correlates in a real-world forced-choice precognition paradigm. 


\section{Method}

\subsection{Participants}

Two hundred and forty-nine participants took part in this study and received entry into a random prize-pool for sixteen $\$ 20$ vouchers (16 participants were readers of The Listener, New Zealand's leading current affairs magazine, while the other 233 participants were first-year Victoria University of Wellington psychology students and received partial course credit). A twotailed power analysis with $\beta=.90$ and $\boldsymbol{r}=.12$ (based on the lower half of most of the confidence intervals found in the meta-analysis of Study I, given that mean effect sizes have a high likelihood of overestimating the true effect; Kennedy, 2016) indicated that at least 725 participants would be required in this study to reach adequate power. We were unable to reach this number, even after multiple attempts to recruit participants both nationwide (through The Listener) and locally at Victoria University of Wellington. The lack of enthusiasm to participate in this study is likely due to the intense timecommitment required ( 8 weeks). This study was granted ethics approval by the Victoria University of Wellington's SoPHEC (School of Psychology Human Ethics Committee).

\subsection{Materials}

All significant predictors found in Study I were included (see below), except for perceptual defensiveness. This is because perceptual defensiveness can only be measured using the Defense Mechanism Test (DMT; Kragh \& Smith, 1970), which is difficult to administer and is very time consuming, requires a tachistoscope, and needs considerable training to be able to correctly interpret participant' drawings (Haraldsson et al., 2002; Meier-Civelli \& Stoll, 1990). Therefore, the DMT was not administered to participants, as it was not practical (it cannot be administered at a distance) or time-efficient.

6.2.1. Extraversion. Extraversion was measured using the two-item subscale in the Ten Item Personality Inventory (TIPI; Gosling et al., 2003). 
The items use a seven-point Likert scale ranging from 1 (not at all like me) to 7 (exactly like me), and include "Extroverted, enthusiastic" and "Reserved, quiet" (reverse scored).

6.2.2. Openness to experience. Openness to experience was measured using the two-item subscale in the TIPI (Gosling et al., 2003). The items use a seven-point Likert scale ranging from 1 (not at all like me) to 7 (exactly like me), and include "Open to new experiences, complex" and "Conventional, uncreative" (reverse scored).

6.2.3. Belief in ESP. Belief in ESP was measured using the exact same question used to measure belief in ESP in Bem's (2011a) study, "Do you believe that ESP exists?" The item uses a five-point Likert scale ranging from 1 (definitely does not) to 5 (definitely does).

6.2.4. Luck belief. Luck belief was measured using a five-item subscale in the Questionnaire of Beliefs about Luck (Luke et al., 2003). It refers to the belief that luck is primarily controllable, and participants who score high in this belief also view luck as internal, stable, and nonrandom. The items use a sevenpoint Likert scale ranging from 1 (strongly disagree) to 7 (strongly agree), and include questions such as "Being optimistic can improve one's luck" and "Pessimism invites bad luck."

6.2.5. Time belief as dynamic. Time belief as dynamic was measured using the seven-item subscale in the Time Metaphor Test (Knapp \& Garbutt, 1958). The subscale consists of seven metaphors that are related to time as being somewhat hasty or dynamic. In total, there are 25 metaphors that participants' place within 5 columns rated from 1 (most appropriate) to 5 (least appropriate). Participants were required to choose exactly five metaphors per column, such that the first five most appropriate phrases would be placed in column one, the next most appropriate phrases in column two, and so on until the five least most appropriate phrases are placed in column five. Examples of such phrases include "a dashing waterfall" and "a fleeing thief." 
6.2.6. Lottery Success. Participants' predictions were compared with the winning NZ Lotto numbers for each Saturday draw to determine lottery performance. As it is likely that not everyone will submit data for all eight weeks, the outcome measure is the percentage of correct hits in relation to the total number of (potential) winning numbers of the weeks' predicted. For example, if participant $\mathrm{X}$ got four numbers correct over six weeks of predictions, the outcome percentage would be $11.11 \%$ (4, the number of hits, divided by 36 , the total number of potential hits for those six weeks).

\subsection{Procedure}

The study was pre-registered with the KPU Study Registry (Zdrenka, 2017) to ensure all analyses were pre-planned (the benefits of doing this are outlined in Chapter Four). Participants first completed an online survey about their beliefs and attitudes. They were then sent a weekly email (for eight weeks) to predict six lotto numbers for each upcoming Saturday draw in the New Zealand lottery.

\section{Results}

As specified in the study's registry document, the data for all participants who completed at least one week (i.e. one lotto draw prediction) were included. Participants who entered duplicate numbers in a single week had all of their guesses excluded for the analysis, which left a total of $N=85$. Table 11 below shows the number of participants who entered their data in any given week (including those who provided invalid data, such as duplicate or missing numbers). Given that there were 5 pre-planned confirmatory analyses to be conducted, the probability that a significant result would be obtained by chance is approximately .23. Therefore, in our registry document we also pre-specified that any result with $\boldsymbol{p} \leq .01$ would be considered a significant outcome whereas a result between .05 and .01 would only be considered suggestive of a relationship (based on the $\boldsymbol{p}=.05$ alpha level being divided by the number of comparisons being made; i.e. .05/5 =.01). 
Table 11

Number of Participants who Submitted Their Predictions Each Week

\begin{tabular}{cc}
\hline Week & Number of Participants \\
\hline 1 & 107 \\
2 & 100 \\
3 & 81 \\
4 & 72 \\
5 & 71 \\
6 & 59 \\
7 & 58 \\
8 & 55 \\
\hline
\end{tabular}

A graph showing the number of participants' and their lottery success can be seen in Figure 37 below. The majority of participants had a 17\% success rate (21 participants), followed by a $0 \%$ success rate (10 participants), and then an $8 \%$ success rate (7 participants). The greatest success rate was $50 \%$ and was achieved by one participant.

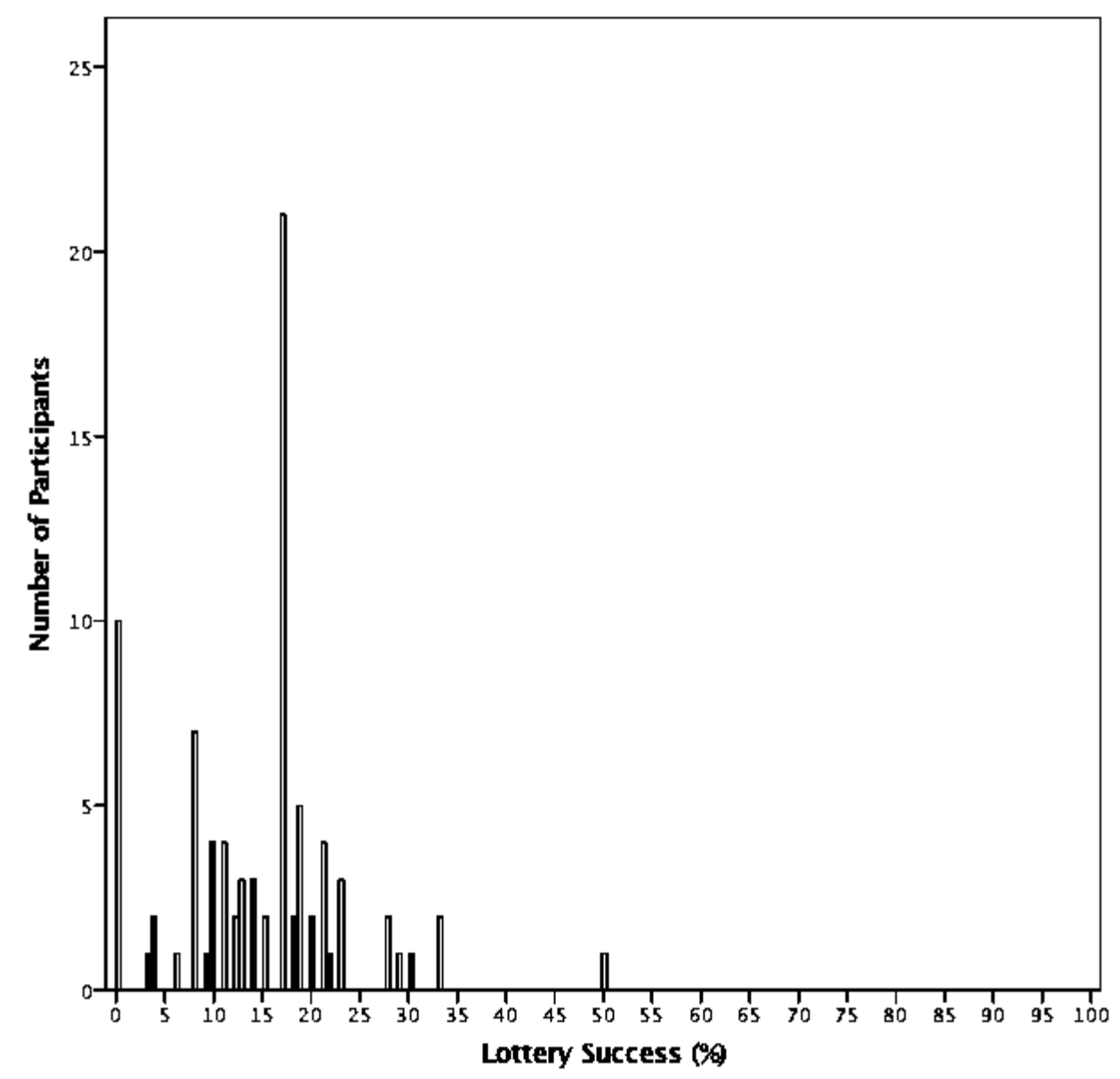

Figure 37. Number of participants and their lottery success percentage. 
The effect sizes we found in Study III(B) can be found in Table 12 below, which compares the findings across all three Studies conducted in this thesis. There were no significant predictors of lottery success: For openness to experience $(r=.13, p=.25)$ and luck belief $(r=.08, p=.48)$, the effect sizes were closely matched with the effect sizes found in Study I. Although the effect size for time belief ( $\boldsymbol{r}=-.10, \boldsymbol{p}=.37$ ) was larger in Study III(B) than the effect size found in Study I, they were both in the same direction. However, the effect sizes for extraversion $(\boldsymbol{r}=-.11, \boldsymbol{p}=.30)$ and belief in $\operatorname{ESP}(\boldsymbol{r}=-.03, \boldsymbol{p}=.82)$ were in the opposite direction to those found in Study I.

Table 12

Effect Size Comparisons for Studies I, II, and III

\begin{tabular}{llll}
\hline Individual Difference Measure & $\begin{array}{l}\text { Study I Effect } \\
\text { Size }(r)\end{array}$ & $\begin{array}{l}\text { Study II Effect } \\
\text { Size }(r)\end{array}$ & $\begin{array}{l}\text { Study III(B) } \\
\text { Effect Size }(r)\end{array}$ \\
\hline Openness to Experience & $.12^{*}$ & -.04 & .13 \\
Extraversion & $.08^{*}$ & .05 & -.11 \\
Belief in ESP & $.13^{*}$ & $.16^{*}$ & -.03 \\
Luck Belief & $.13^{*}$ & & .08 \\
Time Belief & $-.02^{*}$ & & -.10 \\
\hline
\end{tabular}

$* p \leq .05$

Unsurprisingly_due to the low number of participants and the sample size calculated as necessary to detect effects consistent with those identified in Chapter Three-none of the predictor variables significantly correlated with lottery success. While we did plan to run permutation analyses on the data to eliminate potential guessing biases (e.g. participant' preferences for certain numbers or other time/events-dependent guessing habits), this was not necessary given that the study was severely underpowered and the results cannot be taken at face value (randomisation tests would only serve to make the $\boldsymbol{p}$-values even more insignificant due to larger variance for guesses that are not independent). That being said, these biases are less likely to occur in our study given that we are looking at correlations rather than direct hits, and conducting the study over eight weeks with multiple targets (rather than just a single lotto draw; cf. Watt \& Nagtegaal, 2000 and also Study III(A)). 
As suggested in the discussion of Chapter Three, these predictor variables may not be related and may instead be additive, or they may explain common variance (and potentially supress each other), so we performed a multiple regression analysis using the main five measures (belief in ESP, extraversion, openness to experience, luck belief, time belief as dynamic) to predict psi performance. This model did not significantly predict psi performance $\left(\boldsymbol{R}^{2}=.06, F(5,70)=.86, \boldsymbol{p}=.51\right)$. Furthermore, none of the beta-values were individually significant (see Table 13 below). However, this was expected, given that the study was severely underpowered.

Table 13

Multiple Regression Predicting Lottery Success

\begin{tabular}{lrrr}
\hline & $\mathrm{B}$ & $\beta$ & $t$-value \\
\hline Belief in ESP & -1.18 & -.15 & -1.09 \\
Extraversion & -.40 & -.13 & -1.01 \\
Openness to Experience & .55 & .14 & 1.11 \\
Luck Belief & .20 & .16 & 1.18 \\
Time Belief as Dynamic & -.12 & -.07 & -.62 \\
Constant & 13.65 & &
\end{tabular}

All $t$ 's are not significant at $p \leq .05$

\section{Discussion}

Overall, we found no significant predictors of lottery success, along with a lack of consistency between these findings and those of the previous chapters. However, given that we were short 640 participants of our 725 participant' target (to meet power requirements), our study was severely underpowered and the results do not provide much evidential value.

A reverse power analysis was conducted to estimate how large the sample would have needed to be for the effects found in Study III(B) to be significant. Openness to experience (618 participants), extraversion (864 participants), and time belief (1047 participants) would have required a similar amount of participants to that found in our initial power analysis to become significant (725 participants). However, luck belief and belief in ESP would have needed far more participants to reach any level of significance (1638 and 11,671, respectively). While Openness and Time Belief provided effect sizes 
larger than those identified in Chapter Three, the study was underpowered and belief in ESP and extraversion were negatively correlated (cf. Studies I and II in which belief in ESP and extraversion were positively correlated and sufficiently powered), so we can be more confident that the results of Study III(B) are unreliable and will not help inform future power requirements.

Apart from the difficulty of recruiting/observing enough participants, most examples (if not all) of mass forced-choice precognition that naturally occur in the real world only involve a single target, which would make it difficult to locate psi effects amongst the potential noise given that individual guesses are not independent of each other. While some preference biases are easily identifiable (such as people favouring the numbers 7 and 3, or certain personality types favouring certain numbers) there are far more nuanced guessing habits that can make it hard-perhaps even impossible-to account for them all. For example, Goodfellow (1938) re-analysed data from a German radio experiment known as the Zenith tests and found (or inferred) such biases as a sequential effect (people had a preference for a 11211 pattern when there were five consecutive binary trials), unconscious influences from the broadcast itself (e.g. the word 'five' was mentioned multiple times, which led to people favouring the star-which has five ends-over the cross), and general exposures to other stimuli eliciting emotional responses affecting people's guesses for a specific draw. Lotto number biases include preference for numbers related to the current date, the date of the draw, the jackpot prize, and the remaining time until the draw is shown on screen (Wang et al., 2016).

Even though some of these factors are difficult to 'prove' (in the sense that one could posit numerous other possible explanations for these correlations, e.g. a bias for the number 3 may be due to a popular TV show talking about the number 3 rather than anything related to the current date), most researchers would accept these biases as an explanation rather than a psi effect. Therefore, the only way to truly account for all potential guessing biases is to run so many trials that the randomness of all of the lotto draws cancel out any effect that guessing biases might have on a single draw. In other words-no 
systematic patterns of winning numbers should exist once the sample of lotto draws is large enough, and therefore, guessing biases cannot interfere with the results. This was the basis of why we ran eight consecutive trials in Study III(B) rather than a single one, although this is only a starting point and more trials will always be more beneficial. Future research in this area will need to weigh the pros and cons of running a mass test with a single target.

While Study III(B) might have been underpowered, it did give us useful information on the practicality and difficulty of recruiting and retaining participants for a large-scale long-term lotto study that incorporates individual difference assessments. Firstly, our incentives (16 x $\$ 20$ vouchers) might not have been adequate enough for the length of time required of participants (an initial assessment plus 8 week follow up). Secondly, of the 249 participants that filled out the initial assessment, only 85 participants entered one or more lotto draw predictions. This is an almost $2 / 3^{\text {rd }}$ drop-off rate, which may be accounted for by the fact that the 233 Victoria University participants received credit after completing the initial assessment only and likely did not feel obligated to continue with any follow-up lotto draw predictions. Thirdly, only 19 participants completed all 8 trials, which suggests that the weekly voucher draws ( $2 \times \$ 20)$ did not prevent participants from skipping one or more weeks. It may be better to reward participants that complete all 8 trials instead of having a random weekly draw, in order to increase retention rates.

Based on all of these recommendations, specifically regarding the sample size (advice: power analysis), commonality of guessing habits (advice: more trials), and recruiting and retaining participants for a long-term study (advice: greater incentives), future researchers may need to spend a significant amount of time in the planning and preparing stages to avoid the issues that came up in Study III(B). Given the time required of participants and high financial cost to incentivise participants appropriately, such research may be better accomplished through a more structured research programme involving multiple researchers and laboratories (and hopefully some source of funding). Otherwise, we will be left with the same old laboratory research to fall back 
on-unable to satisfactorily deal with the claim that 'if psi were real, casinos would be bankrupt.' 


\section{CHAPTER SIX: CONCLUSION}

This chapter will begin with a summary of the results of the studies in this thesis, followed by a reflection on what these results might mean. I will then conclude with a focus on the bigger picture and philosophical questions that remain in this area of study.

\section{Introduction}

The aim of this thesis is to facilitate one of the long-term foci in parapsychological research-to understand the nature of precognition. This was to be achieved by picking a specific area within parapsychology (forced-choice precognition) and collating all of the research in that area to determine which factors most reliably predict psi success (Study I), followed by systematically testing these results in both an experimental replication (Study II) and a novel real-world study (Study III). The importance of systematic research in parapsychology cannot be overstated, as Alcock (2003) maintains that parapsychology,

...is a field without a core knowledge base, a core set of constructs, a core set of methodologies, and a core set of accepted and demonstrable phenomena that all parapsychologists accept. . . . I consider it doubtful that parapsychologists could agree amongst themselves as to just what experiments or demonstrations in the literature constitute the best case for psi." (p. 32)

Study I's meta-analysis incorporated all available forced-choice precognition experiments (with individual difference measures), and found that 6 of the 23 variables analysed were predictive of psi success: (1) belief in ESP, (2) extraversion, (3) openness to experience, (4) time belief as dynamic, (5) luck belief, and (6) perceptual defensiveness. While the meta-analysis was comprehensive and spanned over 70 years, it also highlighted the lack of systematic research being conducted in this area as the majority of the individual difference variables (88\%) were included in less than 10 studies, 
and/or were the focus of study by fewer than five independent investigators. There is also the risk, with the number of individual meta-analyses conducted in Study I, that one or more of these significant findings may have been an artefact of multiple analyses (e.g. represent a false positive).

Study II examined (1) belief in ESP, (2) extraversion, and (3) openness to experience (a subset of those individual differences identified as potential moderators of performance in Study I), to see whether they could predict psi performance using a previously successful forced-choice precognition experimental paradigm (Bem, 2011a). We found that belief in ESP was significantly related to psi performance $(r=.16, p=.02)$ whereas extraversion and openness to experience were not. However, given that five predictors were analysed in total (including the exploratory analyses of transliminality and magical ideation), a Bonferroni correction-which lowers the significance level to take into account multiple analyses-suggested that even belief in ESP did not meet the threshold of significance in this instance (i.e. $\boldsymbol{p} \leq .01$ was not achieved). Alternatively, one could argue that because Study II was a replication of Bem's (2011a) experiment, we ought to have used a one-tailed test rather than a two-tailed one given that our hypotheses were based on his previous results, and therefore, belief in ESP still meets the adjusted significance levels (just). Study II was also limited by the fact that not all significant predictors were analysed from Study I (since we did not have access to all of the scales at the time of the experiment).

Study III examined these same predictors in a real world lottery study and was pre-registered with the Koestler Parapsychology Unit Study Registry (Zdrenka, 2017). We examined (1) belief in ESP, (2) extraversion, (3) openness to experience, (4) time belief as dynamic, and (5) luck belief, as predictors of lottery success and found that none of the variables significantly predicted psi performance. The sixth significant predictor from Study I (perceptual defensiveness) was not included due to the difficulty of its assessment. Unfortunately, the results were inconclusive as the study was severely underpowered; to meet the power requirements, 725 participants were 
needed but we only managed to get data for 85 participants. We identified two factors which may have contributed to this low rate of participation: (a) the incentives, namely sixteen $\$ 20$ vouchers, may have been insufficient for the time-commitment required, and (b) Victoria University of Wellington participants received course credit after the initial assessment and likely did not feel obligated to continue with the lotto prediction phase of the study. Study III was also limited by the fact that guessing biases could potentially interfere with the interpretation of the results.

In short, the research programme in this thesis started off with promising results, although it ended with a weak finish. One take-away from this is that findings in the laboratory may not translate to meaningful results in the field.

\section{Overall Findings}

So-what do all of these results mean? It would be inappropriate to make any definitive statements about the data given the limitations described above. However, we can interpret the on-again, off-again significant belief in ESP result (or non-significant result, depending on the stance one takes) in a few different ways. The first is simply that the sheep-goat effect does not exist-the significant result can be explained by random variation, which will sometimes produce false positives just by chance. This is supported by the fact that Study II's $\boldsymbol{p}$-value was .02 (rather than < .001), which does not reach significance after the Bonferroni correction is applied. The second interpretation is that the sheep-goat effect does exist, and Study II is confirmation that in a wellcontrolled forced-choice precognition experiment, psi can reliably be detected. This is consistent with previous meta-analyses having found a significant sheepgoat effect in the forced-choice ESP domain (Lawrence, 1993; Storm \& Tressoldi, 2017). It is also supported by the fact that the most robust predictor in forced-choice precognition experiments is belief in ESP as shown in Study I's meta-analysis ( $\boldsymbol{r}=.13, \boldsymbol{p}<.001$; 95\% CI [.07, .20]), having been examined by 12 independent investigators and 24 individual studies. The final interpretation is that the sheep-goat effect exists, but it is not a psi effect; rather, it is an effect 
of 'subjective probability' (Brugger \& Taylor, 2003). This is based on the idea that participants' choices are biased and 'random' target sequences are never truly random - therefore, any patterns exhibited by a participant may correlate with these non-random target sequences, resulting in either psi-hitting or psimissing (Gatlin, 1977, 1979). Consequently, the sheep-goat effect may emerge if 'sheep' and 'goats' differ in their guessing behaviours-and there is support for this, as studies have found that 'sheep' and 'goats' vary in the 'random' choices they make and their judgement of probabilities (Blackmore \& Trościanko, 1985; Brugger et al., 1991).

We will now look at each possibility, along with its implications, in turn.

\subsection{The Null Hypothesis}

The first thing to note is that the only support for psi is through indirect statistical inference and the rejection of the null hypothesis (Hyman, 1995) there are no other observed effects that justify a causal explanation, such as our minds connecting in any way to the outcome (e.g. that which is implied by the term 'precognition'). Otherwise, the success rate in psi experiments (and real life) would be close to $100 \%$, such that people could confidently claim that their thoughts accurately represent a future reality. However, they cannot do so, given that they are wrong most of the time (and are unable to predict when they will be right). In other words, when we are talking about precognition (and individual differences in precognition), we are simply asking the following question: can certain people guess (random) things in the future at an abovechance rate, and if so, what types of people are they? Once we remove these causally presumptuous terms such as "precognition", we are better able to analyse what is (and is not) going on. Think of it this way-people who claim to be better at predicting things in the future, such as lottery numbers, might more aptly be considered lucky (or unlucky if they always lose). In this context, luck is the idea that you can beat the odds, so to speak (and not necessarily through your own actions, but through something beyond your control, a.k.a. 
psi). Therefore, the plausibility of psi hinges on whether one can be luckier than others and effectively beat 'chance'.

To determine whether beating 'chance' is a real possibility, we first need to define the term probability (since beating 'chance' is merely a statistical deviation from a probability estimate). There are two main schools of thought when it comes to probability: the classical frequentist camp (which is what we base most experimental analyses on; Fisher, 1925) and the Bayesian camp (Wagenmakers et al., 2018). The former is based on the idea that if we were to repeatedly (and infinitely) record independent observations using the exact same conditions, the occurrence of each outcome will ultimately match the probability estimate. The latter is an estimate of one's subjective feeling about how likely an event is about to occur. While experts may differ on the meaning of probability, they unanimously agree on the mathematics (e.g. if one assumes that outcome $\boldsymbol{x}$ has a 55\% probability of occurring, then all will agree that the outcome of $\boldsymbol{x}$ 's non-occurrence is 45\%) (Schoenberg, 2016). In a deterministic system, such as the world we live in (notwithstanding the still unresolved contradictions posed by quantum mechanics), nothing is truly 'random'. If we knew everything there was to know about the system in perfect detail, we could predict the future with 100\% accuracy (hence 'chaotic randomness' rather than just randomness; L. Browne, 2015). In other words, all predictions about a future event are either true or false-it either will or will not happen.

However, given that we do not have all of the relevant information about the system at hand, we use probabilities to make use of the information that we do have. So in terms of Bayesian probability estimates, the information we use is based on our past experiences and expectations. Whereas for frequentist probability estimates, we use information about the current conditions that we assume to be true. For example, when determining a frequentist probability estimate for whether a person can predict where a ball will land on a 36 number roulette wheel (with one zero number), we assume that the ball has an equal chance of landing on each number and thus the person has a $2.7 \%$ likelihood of getting it correct. However, this is an 
assumption that can be invalidated if the wheel is biased (e.g. tilted 0.04 degrees and all but unnoticeable to anyone) for example, or if the person making the prediction uses a sophisticated roulette computer hidden from view (which detects the ball trajectory in motion and accurately determines the section of the roulette wheel that the ball will land on). In fact, the only way to determine the truth or falsity of these a priori assumptions is to repeatedly run trials under these exact same conditions to see whether the occurrence of outcomes matches the probability estimate. If it does not match or approach the probability estimate (e.g. $2.7 \%$ ), then the assumptions will necessarily have been false, and need to be adjusted. That is to say that any deviation from 'chance' merely describes the fact that one used an incorrect probability estimate based on incorrect assumptions (Alcock, 1981); nothing more, nothing less. Therefore, it is impossible to 'beat the odds'-the only way that the odds can be beaten is if the odds were incorrect to begin with.

So, if psi is said to emerge in an experiment when participants are lucky or unlucky (i.e. they statistically deviate from the probabilistic estimate) regarding their choices of future random events, what is 'psi' even referring to? As we have shown, frequentist probability estimates are based on assumptions that may or may not be correct, so any above-chance results must mean that the probability model was wrong (or that not enough trials have been run for the long-term average to ultimately converge to its expected value; Borovcnik \& Kapadia, 2014). If we accept this model of reality, psi cannot exist. However, one might argue that psi refers to an above-chance prediction over and above a completely accurate probability estimate, which seems very implausible. Labelling any outcome as a consequence of psi prematurely (i.e. before every non-paranormal way of getting the information has been completely ruled out, and is consistently replicated by independent researchers) seems similarly inappropriate (Hyman, 2017). As an analogy_if a casino owner realises a new patron is consistently winning at roulette, they would do everything they could to find out how the facts were different to what they had assumed prior to the patron winning. The absolute last thing the casino owner would do is accept 
that their probability estimate was a correct depiction of reality, the patron was able to beat chance, and the explanation is paranormal (or outside of any known causal explanation, which is the definition of psi). In parapsychology, the opposite seems to apply, with many researchers quick to claim psi-which is why Alcock (2003) provocatively concludes that "[p]arapsychology is, at bottom, motivated by belief in search of data, rather than data in search of an explanation" (p. 49). Hyman (1995) notes that although some experimental paradigms look promising, he "hasten[s] to add that without further evidence, [he does] not think we can conclude that these effects are all due to the same cause-let alone that they result from a single phenomenon that is paranormal in origin" (p. 342). It seems fair to say that we do not have that evidence yet; we are still looking for a reliable and consistent way to produce psi phenomena upon demand.

The implausibility of psi, on a conceptual level, is one reason to consider the idea that the null hypothesis is 'true'. Another reason to consider the null hypothesis is by understanding how psi differs to other anomalies in the scientific domain. Hyman (2010b) argues that anomalies within science (such as what we now know to be quantum mechanics, or the planet Neptune) typically arise as "byproducts of research aimed at exploring and extending the reach of existing theories" and precisely details how an outcome or observation will deviate from a scientific prediction (p. 50). Consequently, evidence that consistently supports this anomaly leads to wider scientific acceptance, while the lack of such evidence leads to the anomaly being discarded. In contrast, parapsychological research tries to find an anomaly from the outset, and given that psi is both negatively defined and non-falsifiable (in the sense that one can always claim that current conditions were not psi-conducive, and try again ad infinitum), any glitch in a body of data can count as evidence for psi (Hyman, 2010b). The lack of a positive theory of psi, along with the fact that claims of parapsychology often arise outside of the scientific community, allow psi to continue to be seriously investigated even though parapsychology has a long history of inconsistent and non-repeatable claims (Hyman, 2010b). 
Ultimately, claims of psi stem from experiences people have in the real world; the most reported of which are telepathy (direct communication between two minds without using known sensory channels) and precognition (Zusne \& Jones, 1989). The main commonality between the two forms of psi is that they involve judgements of probability (Blackmore \& Trościanko, 1985). For example, you may dream about a unique pair of green and silver shoes that you have never seen before, and a week later, your friend returns back from a trip and gives you those exact shoes as a gift. Whether you decide that it was a coincidence (i.e. chance) or something paranormal (i.e. above-chance, or psi) all comes down to a probability judgement; that is, you are deciding whether this outcome can naturally occur even though the probability of it occurring is so low (in your subjective opinion). As discussed previously, probability estimates are an estimate of reality, and may not mimic the real world unless and until all of your assumptions are correct. Given that probability judgements are notoriously inaccurate (due to our use of heuristics, confidence in erroneous judgements, selective recall, illusion of control, etc.), the most plausible explanation for these real world experiences is a misjudgement of probability that allows one to see normal events as paranormal (Blackmore \& Trościanko, 1985). Precognitive claims in the real world will therefore continue to drive research in parapsychology (cf. anomalies in other scientific domains).

Ultimately, there is a strong case for the null hypothesis being accepted given the implausibility of psi, its non-reproducibility, and its unique position as a negatively defined anomaly often arising outside of the scientific community.

\subsection{The Psi Hypothesis}

There is a clear emphasis placed upon the role of identifying mechanisms in contemporary science, so if psi were real (insofar as above-chance results were achieved at the exclusion of all currently known mechanisms), it does not mean that it is non-explainable. Even if we cannot understand its mechanisms now, in principle, it should be explainable and within the realms of science. In the event that we reliably replicate experimental psi and begin to decipher 
precise conditions for reproducibility, we can create a positive definition of say, precognition, and it will "cease to be a subject of para-psychology [as] such a sense [would have been] discovered," and will no longer be "unknown" (Brugger \& Taylor, 2003, p. 241). At the same time, it is difficult to eliminate all the mundane ways which may cause above-chance results, with Hyman (2017) emphasising that it is practically impossible to control for all of these possibilities and often "when a parapsychologist believes he or she has controlled for every reasonable normal possibility, it later turns out that new normal possibilities are discovered that had not been anticipated" (p. 289). Nevertheless, the possibility remains open that psi-or at least, some currently unknown mechanism—exists, and we will now explore the potential implications of the psi hypothesis being true.

2.2.1. Sheep-goat effect. Schmeidler $(1943,1945)$ first coined the terms 'sheep' and 'goat' in psi research by labelling those who believe in the possibility of ESP as sheep, and those who reject the possibility as goats. Over time, these labels have expanded to include a more broad belief (and disbelief) in some aspect of psychic or paranormal phenomena (Thalbourne, 2010). The 'sheep-goat effect' refers to a significant psi performance difference between the two groups, where 'sheep' tend to perform relatively well in psi tasks and 'goats' perform relatively poorly (Storm, 2016). Palmer's (1972) Vindication Theory attempts to explain this phenomenon as a "need to defend the validity of one's previously formed opinions on important social, moral, and political issues" and can lead to different psi results based on individual attitudes (p. 10). While it logically follows that sheep would be more likely to attend to information that conforms to their beliefs (and therefore perceive psi), it is ironic that the goats' process of rejecting ESP effectively proves its existence through performance below chance (Irwin \& Watt, 2007). This seems to suggest that it is not necessarily that sheep have greater psi ability than goats, but rather, that their differing attitudes reflect how they deal with such information. Lovitts (1981) has provided support for this attitudinal basis for psi, by manipulating the attitudes of participants such that sheep performed worse than chance, while 
goats performed above chance. Participants were split into two groups, a propsi group and an anti-psi group, where the former were told that the experiment was a demonstration of ESP and the latter were told that the experiment was to show that subliminal perception explains ESP. A significant interaction effect was found whereby sheep appeared to have been manipulated to score like traditional goats, and vice versa. Lawrence (1990-1991) has unsuccessfully attempted to replicate this finding. Nevertheless, Wiseman and Smith (2002) showed that people tend to interpret ambiguous stimuli based on cognitive factors (e.g. different thinking styles) rather than motivational factors (e.g. wanting to prove psi), and this may be the basis behind the sheep-goat effect.

2.2.2. Precognition. The sheep-goat question is subsumed within a much larger question: how can anyone (be it a sheep, goat, or even human) perform better than chance when predicting a random event in the future? What can explain precognition? Sceptics claim that it cannot exist, as precognition is incompatible with our current conceptual model of physical reality—so there is nothing that needs to be explained (Bunge, 1987; Crossman, 2009). Researchers have attempted to overcome this objection by embarking on process-oriented research, which is the investigation of the processes by which parapsychological experiences occur, irrespective of its paranormality (Irwin \& Watt, 2007). I will only examine process-oriented precognition research that attempts to explain the psi hypothesis within a scientific framework (i.e. non-paranormal), as there is no reason to assume that its cause, even if currently unknown, lies beyond the reach of scientific understanding (see section 1). The difficulty in assessing such process-oriented research is that, currently, the primary support for psi is through indirect statistical inference and the rejection of the null hypothesis (Hyman, 1995). Therefore, potential theories of precognition are more conceptual or theoretical than evidence based, but this may be a necessary first step to understanding psi if it is truly a legitimate phenomenon. 
2.2.2.1. Quantum theories. Von Lucadao, Romer, and Walach (2007) argue that precognition-and all other forms of psi-are based on quantum entanglement, which is a microscopic state whereby one observed measurement of a component (e.g. subatomic particle) is correlated with a second observed measurement of a different component, even if they are so far apart that it would be impossible for them to be in traditional communication with one another. Therefore, precognition (or specifically certain measurements of belief, such as the measurement of ESP belief and its correlation with psi success) can be explained as some form of entanglement with targets and the mind. However, this view of psi uses a liberal definition of quantum theory known as Weak or Generalized Quantum Theory (Atmanspacher, Römer, \& Walach, 2002), which applies quantum effects in the microscopic level to general systems on the macroscopic level (such as conscious individuals) without much justification or evidence. It effectively applies a framework to Carl Jung's (1973) 'synchronicity' hypothesis, where two meaningful but not causally connected events occur together. It is difficult to examine these acausal explanations of psi from a scientific point of view, since our reductionist worldview is predicated upon cause and effect; it is therefore more akin to a metaphor than a genuine model of psi. Nonetheless, Bem (2011a) argues that since quantum mechanics and alternative conceptions of time and causality in physics do not discount the possibility of precognition, we ought to take the claim seriously.

2.2.2. Real-time ESP. Some researchers theorise that precognitive effects may arise from unconscious use of real-time ESP (such as clairvoyance), as participants could use their own ESP to gather information about the experimenter or test equipment which could enable them to score above chance (R. L. Morris, 1980; Mundle, 1964). In real life, then, people may make predictions about the future that turn out correct, precisely because they had used ESP to notice cues or circumstances in the current environment in order to make an accurate inference about the future (e.g. noticing the deterioration of a car's engine via ESP and consequently predicting a malfunction or car crash in 
the near future). While such an explanation might remove the major theoretical issues of precognition, such as time reversal (J. Taylor, 1980), it merely replaces one incomprehensible mechanism (based on our current scientific understanding of the world) with another-albeit one that is intuitively more understandable than reverse causality.

A full examination of other forms of ESP is beyond the scope of this thesis (but see Hyman, 1995; and Ullman, Krippner, \& Vaughan, 1989). However, we can compare the effect sizes of clairvoyance experiments with precognition experiments to determine the plausibility of this explanation for precognition; if the real-time ESP hypothesis is supported, larger effect sizes should be found in clairvoyance experiments compared to precognition experiments, since an extra calculative step is needed for precognition (as you have to make an inference from the present circumstance to a future outcome). However, Steinkamp, Milton, and Morris (1998) compared the effect sizes of all forced-choice precognition experiments with clairvoyance experiments and report no statistically significant differences, which casts doubt on this theoretical model of precognition.

2.2.2.3. Multiphasic Model of Precognition. According to Marwaha and May (2015), the Multiphasic Model of Precognition (MMPC) is capable of addressing existing experimental psi data. The MMPC essentially splits the problem of precognition into two independent domains: (1) the physics domain, and (2) the neuroscience domain, with the former referring to how information can subvert space-time constraints while the latter refers to the processes by which such information can be perceived by the human mind and lead to a precognitive response. They argue that we do not need to be concerned with the reverse-causality implications when analysing the neuroscience domain; what is important is just that the information-which may or may not be from the future-is available in the present (Marwaha \& May, 2015). For the neuroscience domain, two hypotheses have been proposed to explain how such information signals might be processed: (1) psychophysical variability in a signal transducer, which can account for individual differences 
in psi ability, and (2) a cortical hyperassociative mechanism, which is the processing of a signal via a crossmodal mechanism (i.e. perception involving interactions between two or more different sensory modalities, such as that which occurs for people with synaesthesia). For the physics domain, May and Depp (2015) suggest that the solution might be found within entropic considerations (relating to an increased uncertainty or lack of information about a system). They argue that psi performance correlates with increased entropic gradients of target stimuli (e.g. a high entropy state would be a state that is highly disorganised from an information systems point of view, such as a nuclear test), and the second law of dynamics-when related to changes of entropy — can account for the apparent paradox of time between the microscopic perspective (where equations of motion are all symmetric in time) and the macroscopic perspective (where time appears unidirectional). May and Depp (2015) do acknowledge, however, that the actual mechanism underlying precognition remains unclear.

2.2.2.4. Psi Mediated Instrumental Response Model. Just as humans possess a variety of faculties that are adaptive in managing threats in the environment, or enhancing reproductive success (e.g. Gluckman, Hanson, \& Spencer, 2005; Neuberg, Kenrick, \& Schaller, 2011), Stanford (1974) speculates that psi may be part of a system that adaptively gathers useful information to serve human needs-although what these needs are remain rather vague. The Psi Mediated Instrumental Response Model (PMIR) has been subsequently elaborated (Stanford, 1977, 1982, 1990) and suggests that psi can operate unconsciously (or non-intentionally) to produce advantageous outcomes for an organism, by triggering pre-existing behaviours, memories, feelings, or desires in response to opportunities or threats in the environment. A similar explanation was used by Bem (2011a) to justify his hypothesis for the Precognitive Detection of Erotic Stimuli experiment, where it was argued that anticipating erotic images would be evolutionarily advantageous for reproduction or survival. For example, one may non-intentionally follow an impulse that leads to a beneficial outcome in real life (Watt \& Nagtegaal, 
2000). While PMIR might provide a basis for humans having developed a psi

ability (and even variation in the distribution of this faculty across the population, in line with the sheep-goat hypothesis), it is still not specific enough to formulate the conditions in which psi could not occur, nor does it provide the mechanism by which precognition actually operates.

Clearly, there is a great deal of speculation concerning how precognition works from both a conceptual and theoretical level, but ultimately there is still no known mechanism for precognition at present (Houran, Lange, \& Hooper, 2018). Nevertheless, the issue will not be resolved through logical analysis, but rather, by way of empirical investigation (Irwin \& Watt, 2007). We must therefore go wherever the data takes us.

\subsection{Psi As Subjective Probability.}

A final interpretation of the data presented in this thesis is that the sheep-goat effect is caused by participants' subjective randomness biases. Brugger and Taylor (2003) attempted to relabel ESP as an Effect of Subjective Probability, on the basis that target sequences are always generated using an algorithm (which will generate sequences that are more or less patterned), and therefore, participants' response preferences can easily coincide with target symbol patterning. Following this reasoning, the sheep-goat effect may arise when we compare groups of individuals who share a similarity with others (e.g. belief or disbelief in ESP) and who also demonstrate different types of sequential guessing behaviours, as each group may either psi-hit or psi-miss collectively (Brugger \& Taylor, 2003). There is evidence to suggest that there are systematic differences in appreciation and generation of randomness that is consistent with differential matching performances of pseudorandom targets (e.g. Blackmore \& Trościanko, 1985; Brugger, Landis, \& Regard, 1990). For example, sheep are more likely than goats to avoid repeating the same guesses (Brugger et al., 1990). This does not necessarily explain why sheep are more likely to psi-hit, and goats psi-miss (cf. vice versa), but some studies have shown a reversal of this effect as well (e.g. Lovitts, 1981). 
Proponents of psi might argue that while differences in subjective randomness might exist between groups, in actual experimental psi tasks sheep use psi to make their choices (rather than attempting to make 'random' guesses). Nevertheless, Brugger and Taylor (2003) claim that all parapsychological results are due to subjective probability, such that psi will be observed "when the inherent biases in two sequences [the RNG output and the participants' responses] are congruous" (p. 241). They therefore argue that the way forward for parapsychology is not to dispose of the field, but rather, to transition from a causal interpretation of psi (e.g. your mind accessing information) to a correlational one. Guessing behaviours, such as the process of anticipation and learning of sequential restraints, are all important areas of research in behavioural science (e.g. Bischoff-Grethe, Proper, Mao, Daniels, \& Berns, 2000; Huettel, Mack, \& Mccarthy, 2002) that could benefit from new data in the matching paradigms of parapsychology. If we take this approach, it may be particularly pertinent to examine the individual differences in the attempted randomisation of guesses so that we can better understand the sheep-goat effect.

\section{The Non-Significant Results}

All of the data in Studies I, II, and III, could be re-analysed post-hoc to explore other (non-hypothesised) relationships, unexpected patterns, and alternative psi effects. For example, I considered analysing all of the Big-Five measures after collecting the data for Study II, as well as re-analysing the data collected for Study III in search of the displacement effect (see below)— however, this was not done for good reason. While this thesis set out to examine whether psi phenomena could reliably be produced, I also-and perhaps more importantly — wanted to take seriously the possibility that the null hypothesis is true. Parapsychologists have been criticised for their quick dismissal of non-significant findings as post-hoc, showing some other psi effect (Alcock, 2003), with Richard Wiseman (2010) labelling the problem as "heads I win, tails you lose" (para 1.). I did not want to add to that problem. To understand how prevalent this is in parapsychology, we need only take a look 
at the list of [often post-hoc] labels that parapsychologists have used to describe the different manifestations of psi in the past. The most commonly accepted and widely used is the psi-experimenter effect (Parker \& Millar, 2014; M. D. Smith, 2003), whereby significant findings cannot be replicated due to researchers' lack of psi conduciveness (cf. the original experimenter/s, who were psi conducive). While there is some evidence to this (e.g. Watt \& Ramakers, 2003; Wiseman \& Schlitz, 1997), this phenomenon is as testable as psi itself. One would merely need to run a similar study as Wiseman and Schlitz (1997) did-where both authors ran separate experiments (but in the same location, with the same equipment/procedures and subject pool)—and use a valid and reliable measure of ESP belief (or actual testing of psi ability) for both researchers beforehand. A few pre-registered studies from multiple laboratories that reliably predict success based on researchers' psi beliefs would make a strong case for the existence of psi, for sceptic and proponent (well, maybe not all sceptics). However, before the evidence is in, it does not make sense to attribute a failed replication attempt to a psi-experimenter effect when this lack of consistency and reliability is more in line with the null hypothesis than anything else (otherwise, how can we explain failed experiments by pro-psi researchers? —of which there are many).

The next list of 'effects' will be discussed more briefly, as they are less widely used after failing to be replicated over the long run. The first is the decline effect, where results are initially psi-indicative but fade away over time (e.g. Colborn, 2004). In the same category is the incline effect, the opposite of the decline effect-but of course, this momentum does not continue or it would show an overall psi effect, and the rebound effect, which is a U-shaped curve to describe effect sizes starting strong, dissipating over time, and then returning back to its initial strength (e.g. Bierman, 2001). The quartile-decline effect is specifically related to scores being recorded in two columns on a page, such that psi success significantly decreases when comparing the top left quadrant on the page to the bottom right quadrant (discussed in Alcock, 2003). Another common effect is the displacement effect, which is the unintentional psi-hitting 
or psi-missing of targets that are usually one above or below in the current target sequence (Braud, 1987; Mangan, 1955, 1957), whereas the psi-missing displacement effect is the same as the displacement effect but only for participants who psi-miss in the main task (e.g. Crandall \& Hite, 1983). Psi may sometimes also 'appear' via large spikes not in the actual scores but in the variance of the scores of the individual participants, known as a high-subject variance effect (e.g. Buzby, 1967b, 1967a), while greater run-score variance in the first half of a test compared to the second is termed the decline of variance effect (e.g. Carpenter, 1968; Rogers \& Carpenter, 1966). Similarly, when participants psi-hit and psi-miss within the same trial run it is known as a psidifferential effect, despite the overall hit rate not being significant (e.g. Freeman, 1966). There is also the response bias effect, where lesser-chosen responses are more likely to be hits than frequently chosen responses (e.g. Kanthamani \& Rao, 1975; Stanford, 1973). A less common one is the checker effect, where participants' score significantly better in a psi task if they correctly predict who is going to check/mark their data compared to those who do not (e.g. S. Feather \& Brier, 1968; O'Brien, 1979). Finally, there is the Rao Reversal, which is the reversal of previous scoring direction found in a replication attempt (e.g. Rao, 1963), similar to the Midas Touch whereby an original psi experiment cannot be replicated by the same experimenter due to the experimenter (supposedly) losing interest after the initial success (e.g. Taves \& Dale, 1943).

The point of listing all these terms is not to discredit or dismiss them (as there is nothing wrong with exploratory analyses and labelling of patterns, per se) but to show that parapsychological research has been rife with the continuous creation, and discarding, of old labels that no longer fit the data, without adequate and systematic testing of such effects-in isolation, not just running normal psi experiments and searching the data for every alternative way psi could have manifested. While it is possible that psi is so complex that post-hoc exploration is necessary, this could go on forever in a field like parapsychology as studies cannot disprove psi (as we are unable to know when 
psi is absent and therefore have no adequate controls-it is only negatively defined in terms of ruling out 'normal' explanations). Therefore, all studies provide opportunities to extend psi theories post-hoc. If one accepts that all of these effects are possible manifestations of psi, consider two examples of an experimental outcome: (1) a standard above-chance psi result in an experiment, supporting the psi hypothesis, yet the lack of all of these other psi effects in the data is not seen as detrimental to the psi hypothesis at all (Alcock, 2003), or (2) an unintended psi effect such as the displacement effect, which would also support the psi hypothesis. As you can see, it is a catch-22 situation-no outcome will be taken to support the null hypothesis (Hyman, 1995). We need to define precisely the conditions in which an effect can, and cannot, occur for it to have evidential value; otherwise, an effect that is not predictable beforehand should merely be seen as random variation consistent with the null hypothesis. This is the reason why I have been so strict in my exploration of the data and analyses in this thesis-the last thing I want to do is resurrect any of these discarded effects post-hoc and continue this never-ending cycle.

This also ties back in with the replication crisis that social psychology, in particular, has been hit with recently (see Chapter Four). The replication crisis did not necessarily arise from egregious practices, but more so from everyday practices in science that allow a few extra researcher' degrees of freedom here and there which can inadvertently lead to false positive outcomes (Simmons et al., 2011; Wagenmakers et al., 2011). This methodological flexibility likely plays an important role in parapsychology too-and just as the Open Science Collaboration project (Open Science Collaboration et al., 2015) was unable to replicate the majority of classic psychology studies, so too are most of these post-hoc parapsychological effects irreproducible. To move forward in both fields, pre-registration needs to become commonplace, so that it becomes clear which studies provide replicatory evidence and which studies merely provide exploratory hypotheses for the future. 


\section{Final Thoughts}

I have attempted to answer the following question in this thesis: is precognition real (yet hard to pin down) and therefore requires further research to isolate/make it more reliably evident (e.g. using forced-choice precognition correlates), or is the evidence for precognition 'simply' a set of false positives reflecting methodological weaknesses, chance, and biases in the research process (Franklin et al., 2014)? The benefits of exploring the forced-choice precognition paradigm is that it is far less prone to sensory leakage than any other parapsychological paradigm, as targets are generated after the participant makes their 'precognitive' choice (Mossbridge \& Radin, 2018). This does not mean that outcomes cannot be biased in other ways-as seen by the criticisms of Bem's (2011a) studies in Chapter Four-but it is one less factor to muddy the waters. Given that psi is extremely elusive and has yet to be reliably detected (from both an examination of the literature and the studies conducted in this thesis), I am inclined to give the benefit of the doubt to the null hypothesis until further evidence becomes available.

Over the course of this thesis, I have learnt that running a parapsychological study is far easier in my head (I mean, it is just getting people to guess an RNG, right?) than in practice. There are so many variables that one needs to account for, such as zero possibility for sensory leakage, perfect randomisation methods, controlling for people's guessing biases, appropriate motivation/incentives for participants, adequate power and sample size to detect small effects, correcting for multiple analyses, pre-planning hypotheses based on past research (much of which is unstructured and inconsistent), pre-registration (I could go on...). Not to mention the philosophical implications that I have had to continuously ask myself: (1) what does it mean if psi is real-as in, how is it possible for any study to control for anything if psi can influence anyone at any time? (2) can precognition really exist if, in knowing the future, you can prevent the future? (3) if probabilities are an approximation of reality, and psi allows us to beat this approximation, 
does that mean our probability estimates are wrong or that psi has a truly remarkable ability to affect our probabilistic laws in an unknown way?

Suffice to say, I still have many unresolved questions, and will keep my eye out in this space.

Ultimately, the biggest challenge parapsychology faces at present is to find an experiment that can be demonstrated by any researcher, or at the very least, experimenters of a clearly defined type (Steinkamp, 2005). To assist in this goal, promising paradigms need to be systematically explored by multiple researchers using pre-registered studies and prospective meta-analyses (Kennedy, 2015; Watt, 2005). Otherwise, psi proponents will continue to claim that their studies have 'proven' psi, while sceptics will claim that nothing has changed over the last century of psi research (although, many researchers may not have access to out of print or rare periodicals in parapsychology; Alvarado, 1982). Designing such a study is not an easy task, as small effect sizes typical in this area mean that a definitive and replicable study will require a large sample size and need to be tightly controlled (in fact, if precognition was real, its effect size would need to be small and hard to detect, otherwise people would try to alter their future causing a paradox: see Chapter One).

If I were able to run just one more precognition study and had unlimited resources to do so, I would opt again for another lottery study looking at the sheep-goat effect, as I believe it has the best potential to show us whether precognitive effects really occur in the real world. The first thing I would do is set a fixed length of time for the study: in this case, two years. This would equate to 104 weekly lottery draws, providing a large enough sample of targets to mitigate the effects of a per-draw shared target set (see Chapter Five). I would then work with the government to make it mandatory for all lotto purchasers to fill out a sheep-goat measure, and consequently observe the predictions of all lotto players for the next two years (specifically those who choose their own numbers). I would also set up a variety of RNGs and PRNGs to automatically give a 'random' output of lotto numbers every week, to act as a 
control. Once the two years are over, I would analyse the data as follows: (1) the sheep-goat measure predicting lottery success, (2) all winning numbers over this period, to confirm that the distribution approximates MCE and was not biased, and (3) the hit rate of the RNG and PRNG outputs to winning numbers, compared with the MCE. All of these details would be pre-registered prior to the commencement of this study. If the sheep-goat measure significantly predicted lottery success and the winning numbers were equally distributed as expected by MCE and the RNGs and PRNGs were no better than chance at predicting winning numbers, then that would be highly suggestive of a precognitive effect. Any other result would be taken to support the null hypothesis.

Although a rather quixotic example above, if parapsychology is to be treated with respect by the scientific community, it is essential that exemplar studies come to light that scientists can repeat and use to better understand the principles, procedures, methods, and standards in the field of parapsychology (French, 2004; Hyman, 1995). This also means parapsychologists need to explore psi within a scientific framework (and label it, at most, as an atypical ability) rather than as a spiritual, supernatural, or paranormal phenomenon (Marwaha \& May, 2015). Parapsychology as appropriately defined, is the scientific study of the actual bases of anomalous experience (Irwin, 1989), lest it become the "anomalous study of the anomalous" (Bunge, 1987, p. 576). Overall, I have faith that we will eventually be pulled in the right direction; and I am still open to this being caused by a future event. 


\section{REFERENCES}

Aarnio, K., \& Lindeman, M. (2007). Religious people and paranormal believers: Alike or different? Journal of Individual Differences, 28(1), 1-9. https://doi.org/10.1027/1614-0001.28.1.1

Adams, P. M. (2014). The healing field: Energy, consciousness and transformation. Bloomington, IN: Balboa Press.

Akers, C. (1985). Can meta-analysis resolve the ESP controversy? In P. Kurtz (Ed.), A skeptic's handbook of parapsychology. Buffalo, NY: Prometheus.

Alcock, J. (1981). Parapsychology, science or magic? a psychological perspective (1st ed.). Oxford, NY: Pergamon Press.

Alcock, J. (1987). Parapsychology: Science of the anomalous or search for the soul? Behavioral and Brain Sciences, 10(4), 553-565. https://doi.org/10.1017/S0140525X00054467

Alcock, J. (2003). Give the null hypothesis a chance: Reasons to remain doubtful about the existence of Psi. Journal of Consciousness Studies, 10(F0020006), 29-50.

Alcock, J. (2011). Back from the future: Parapsychology and the Bem affair. Retrieved from https://www.csicop.org/specialarticles/show/\%20back_from_the_future

Alvarado, C. S. (1982). Historical perspective in parapsychology: Some practical considerations. Journal of the Society for Psychical Research, 51, 265-271.

Ashe, G. (2001). Encyclopedia of prophecy. Santa Barbara, CA: ABC-CLIO.

Atmanspacher, H., Römer, H., \& Walach, H. (2002). Weak quantum theory: Complementarity and entanglement in physics and beyond. Foundations of Physics, 32(3), 379-406. https://doi.org/10.1023/A:1014809312397

Ayton, P., \& Fischer, I. (2004). The hot hand fallacy and the gambler's fallacy: Two faces of subjective randomness? Memory \& Cognition, 32(8), 1369-1378. https://doi.org/10.3758/BF03206327

Bacon, F. (1909). Essays or counsels: Civil and moral. In C. Eliot (Ed.), Harvard classics volume 3: Essays, civil and moral, and new atlantis; Areopagitica and tractate of education; Religio medici. New York: P. F. Collier \& Son.

Baker, R. A. (1992). Hidden memories: Voices and visions from within. Buffalo, NY: Prometheus Books. 
Bargh, J. A., \& Ferguson, M. J. (2000). Beyond Behaviorism: On the Automaticity of Higher Mental Processes. Psychological Bulletin, 126(6), 925-945. https://doi.org/10.1037/0033-2909.126.6.925

Bargh, J. A., \& Morsella, E. (2008). The unconscious mind. Perspectives on Psychological Science, 3(1), 73-79. https://doi.org/10.1111/j.17456916.2008.00064.x

Baron, R. M., \& Kenny, D. A. (1986). The moderator-mediator variable distinction in social psychological research: Conceptual, strategic, and statistical considerations. Journal of Personality and Social Psychology, 51(6), 11731182. https://doi.org/10.1037/0022-3514.51.6.1173

Bates, C., \& Stevens, D. (1989). Illusion and well-being: Toward a new agenda for mental health. Journal of Counseling and Development, 67(10), 590-591. https://doi.org/10.1002/j.1556-6676.1989.tb01337.x

Bauer, H. H. (2014). Anomalistics, pseudo-science, junk science, denialism: Corollaries of the role of science in society. Journal of Scientific Exploration, $28(1), 95-111$.

Becker, E. (1997). The denial of death. New York: Free Press.

Begley, C. G., \& Ellis, L. M. (2012). Drug development: Raise standards for preclinical cancer research. Nature, 483(7391), 531-533. https://doi.org/10.1038/483531a

Bem, D. J. (2003). Precognitive habituation: Replicable evidence for a process of anomalous cognition. In Proceedings of Presented Papers: The Parapsychological Association 46th Annual Convention (pp. 6-20). Retrieved from https://pdfs.semanticscholar.org/8033/f0406daadc956c18d847cb39afc1610b2e 73.pdf

Bem, D. J. (2011a). Feeling the future: Experimental evidence for anomalous retroactive influences on cognition and affect. Journal of Personality and Social Psychology, 100(3), 407-425. https://doi.org/10.1037/a0021524

Bem, D. J. (2011b). Response to Alcock's “Back from the Future: Comments on Bem". Retrieved from https://www.csicop.org/specialarticles/show/response_to_alcocks_back_from_t he_future_comments_on_bem 
Bem, D. J. (2012). Bem's response to Ritchie, Wiseman, and French. Retrieved from http://journals.plos.org/plosone/article/comment?id=info:doi/10.1371/annotatio n/02eae6d6-af7f-41d8-b2b3-b6d32fdce7a6

Bem, D. J., \& Honorton, C. (1994). Does Psi exist? Replicable evidence for an anomalous process of information transfer. Psychological Bulletin, 115(1), 418. https://doi.org/10.1037/0033-2909.115.1.4

Bem, D. J., Tressoldi, P., Rabeyron, T., \& Duggan, M. (2015). Feeling the future: A meta-analysis of 90 experiments on the anomalous anticipation of random future events. F1000Research, 4, 1188. https://doi.org/10.12688/f1000research.7177.1

Bem, D. J., Utts, J., \& Johnson, W. O. (2011). Must psychologists change the way they analyze their data? Journal of Personality and Social Psychology, 101(4), 716719. https://doi.org/10.1037/a0024777

Bhadra, B. (1966). The relationship of test scores to belief in ESP. Journal of Parapsychology, 30(1), 1-17.

Bierman, D. J. (2001). On the nature of anomalous phenomena: Another reality between the world of subjective consciousness and the objective world of physics. In P. Van Loocke (Ed.), The physical nature of consciousness (pp. 269-292). Amsterdam, Netherlands: John Benjamins Publishing Company.

Bierman, D. J. (2011). Anomalous switching of the bi-stable percept of a Necker cube: A preliminary study. Journal of Scientific Exploration, 25(4), 721.

Bierman, D. J., \& Bijl, A. (2014). Anomalous 'retrocausal' effects on performance in a Go/NoGo task. Journal of Scientific Exploration, 28(3), 437-452.

Bierman, D. J., \& Radin, D. I. (1997). Anomalous anticipatory response on randomized future conditions. Perceptual and Motor Skills, 84(2), 689-690. https://doi.org/10.2466/pms.1997.84.2.689

Bierman, D. J., \& Scholte, H. S. (2002). A fMRI brain imaging study of presentiment. Journal of International Society of Life Information Science, 20(2), 380-388.

Bischoff-Grethe, A., Proper, S., Mao, H., Daniels, K., \& Berns, G. (2000). Conscious and unconscious processing of nonverbal predictability in Wernicke's area. Journal of Neuroscience, 20(5), 1975-1981. https://doi.org/10.1523/JNEUROSCI.20-05-01975.2000

Blackmore, S., \& Trościanko, T. (1985). Belief in the paranormal: Probability judgements, illusory control, and the 'chance baseline shift'. British Journal of 
Psychology, 76(4), 459-468. https://doi.org/10.1111/j.2044-

8295.1985.tb01969.x

Borenstein, M., Hedges, L. V., Higgins, J. P. T., \& Rothstein, H. R. (2005).

Comprehensive meta-analysis (Version 2) [Computer Software]. Englewood, NJ: Biostat.

Borenstein, M., Hedges, L. V., Higgins, J. P. T., \& Rothstein, H. R. (2009).

Introduction to meta-analysis (1st ed.). Chichester, U.K: Wiley.

Borenstein, M., Hedges, L. V., Higgins, J. P. T., \& Rothstein, H. R. (2010). A basic introduction to fixed - effect and random - effects models for meta - analysis. Research Synthesis Methods, 1(2), 97-111. https://doi.org/10.1002/jrsm.12

Borovcnik, M., \& Kapadia, R. (2014). From puzzles and paradoxes to concepts in probability. In E. J. Chernoff \& B. Sriraman (Eds.), Probabilistic thinking presenting plural perspectives (pp. 35-73). Dordrecht, Netherlands: Springer.

Braud, W. (1987). Dealing with displacement. Journal of the American Society for Psychical Research, 81(3), 209-231.

Brier, B. (1974). Precognition and the philosophy of science. New York: Humanities Press.

Broderick, D. (1992). The lotto effect: Towards a technology of the paranormal. Hawthorn, Vic: Hudson Publishing.

Broughton, R. S. (1991). Parapsychology: The controversial science. New York: Ballantine Books.

Browne, B. A., \& Brown, D. J. (1994). Predictors of Lottery Gambling Among American College Students. Journal of Social Psychology, 134(3), 339-347. https://doi.org/10.1080/00224545.1994.9711738

Browne, L. (2015). Some difficulties in coincidence analysis. Australian Journal of Parapsychology, 15(1), 53-79.

Brugger, P., Landis, T., \& Regard, M. (1990). A 'sheep-goat effect'in repetition avoidance: Extra-sensory perception as an effect of subjective probability? British Journal of Psychology, 81(4), 455-468.

Brugger, P., Regard, M., \& Landis, T. (1991). Belief in extrasensory perception and illusory control: A replication. Journal of Psychology, 125(4), 501-502. https://doi.org/10.1080/00223980.1991.10543314 
Brugger, P., \& Taylor, K. I. (2003). ESP: Extrasensory perception or effect of subjective probability? Journal of Consciousness Studies, 10(6-7), 221-246.

Bullock, R. J., \& Svyantek, D. J. (1985). Analyzing meta-analysis: Potential problems, an unsuccessful replication, and evaluation criteria. Journal of Applied Psychology, 70(1), 108-115. https://doi.org/10.1037/0021-9010.70.1.108

Bunge, M. (1987). Why parapsychology cannot become a science [Peer commentary on "The anomaly called psi: Recent research and criticism," by K. R. Rao \& J. Palmer]. Behavioral and Brain Sciences, 10(4), 576-577. https://doi.org/10.1017/S0140525X00054595

Bunge, M. (1991). A skeptic's beliefs and disbeliefs. New Ideas in Psychology, 9(2), 131-149. https://doi.org/10.1016/0732-118X(91)90017-G

Buzby, D. (1967a). Precognition and a test of sensory perception. Journal of Parapsychology, 31(2), 135-142.

Buzby, D. (1967b). Subject attitude and score variance in ESP tests. Journal of Parapsychology, 31(1), 43-50.

Camp, B. H. (1937). (Statement in Notes Section.). Journal of Parapsychology, 1, 305.

Cardeña, E., Lynn, S. J., \& Krippner, S. (2013). Varieties of anomalous experience: Examining the scientific evidence. Washington, DC: American Psychological Association.

Carey, B. (2011). Journal's paper on ESP expected to prompt outrage. Retrieved from http://www.nytimes.com/2011/01/06/science/06esp.html

Carpenter, J. (1968). Two related studies on mood and precognition run-score variance. Journal of Parapsychology, 32(2), 75-89.

Cattell, H. E. P. (1996). The original big five: A historical perspective. European Review of Applied Psychology, 46, 5-14.

Cattell, H. E. P., \& Mead, A. D. (2008). The sixteen personality factor questionnaire (16PF). In G. Boyle, G. Matthews, \& D. Saklofske (Eds.), The SAGE handbook of personality theory and assessment (Vol. 2, pp. 135-178). Trowbridge, UK: Cromwell Press.

Cermak, T. L., \& Rosenfeld, A. A. (1987). Therapeutic considerations with adult children of alcoholics. Advances in Alcohol \& Substance Abuse, 6(4), 17-32. https://doi.org/10.1300/J251v06n04_03

Chinnery, M. A. (1988). Solar geophysical data comprehensive reports: Data for July 1988. Retrieved from 
ftp://ftp.ngdc.noaa.gov/STP/SOLAR_DATA/SGD_PDFversion/1989/sgd8901c .pdf

Chotai, J., \& Wiseman, R. (2005). Born lucky? The relationship between feeling lucky and month of birth. Personality and Individual Differences, 39(8), 1451-1460. https://doi.org/10.1016/j.paid.2005.06.012

Colborn, M. (2004). The decline effect in spontaneous and experimental psychical research. Journal of the Society for Psychical Research, 71, 1-21.

Collins, H. M., \& Pinch, T. J. (1979). The construction of the paranormal: Nothing unscientific is happening. The Sociological Review, 27(1_suppl), 237-270. https://doi.org/10.1111/j.1467-954X.1979.tb00064.x

Coover, J. E. (1917). Experiments in psychical research at Leland Stanford Junior University. Stanford University, Calif.

Costa, P. T., \& McCrae, R. R. (1992). Revised NEO Personality Inventory (NEO-PI-R) and NEO Five-Factor Inventory (NEO-FFI) manual. Odessa, FL:

Psychological Assessment Resources.

Crandall, J. E., \& Hite, D. D. (1983). Psi-missing and displacement: Evidence for improperly focused psi? Journal of the American Society for Psychical Research, 77(3), 209-228.

Crossman, A. (2009). Parapsychology: the control group for science. Retrieved from https://www.lesswrong.com/posts/enuGsZoFLR4KyEx3n/parapsychology-thecontrol-group-for-science

Del Prete, G., \& Tressoldi, P. E. (2005). Anomalous cognition in hypnagogic state with OBE induction: An experimental study. Journal of Parapsychology, 69(2), 329-339.

Dijksterhuis, A., \& Smith, P. K. (2002). Affective habituation: subliminal exposure to extreme stimuli decreases their extremity. Emotion, 2(3), 203-214. https://doi.org/10.1037//1528-3542.2.3.203

Drewes, A. A. (2002). Dr. Louisa Rhine's letters revisited: The children. Journal of Parapsychology, 66(4), 343-370.

Drucker, S. A., Drewes, A. A., \& Rubin, L. (1977). ESP in relation to cognitive development and IQ in young children. Journal of the American Society for Psychical Research, 71, 289-298. 
Duane, T., \& Behrendt, T. (1965). Extrasensory electroencephalographic induction between identical twins. Science, 150(3694), 367. https://doi.org/10.1126/science.150.3694.367

Dunn, L. M., \& Hottel, J. V. (1961). Peabody picture vocabulary test performance of trainable mentally retarded children. American Journal of Mental Deficiency, $65,448-452$.

Dunne, B. J., Nelson, R. D., \& Jahn, R. G. (1988). Operator-related anomalies in a random mechanical cascade. Journal of Scientific Exploration, 2(2), 155-179.

Duval, S., \& Tweedie, R. (2000). Trim and fill: a simple funnel-plot-based method of testing and adjusting for publication bias in meta-analysis. Biometrics, 56(2), 455-463. https://doi.org/10.1111/j.0006-341X.2000.00455.x

Eckblad, M., \& Chapman, L. J. (1983). Magical ideation as an indicator of schizotypy. Journal of Consulting and Clinical Psychology, 51(2), 215-225. https://doi.org/10.1037/0022-006X.51.2.215

Egger, M., Smith, G. D., Schneider, M., \& Minder, C. (1997). Bias in meta-analysis detected by a simple, graphical test. British Medical Journal, 315(7109), 629634. https://doi.org/10.1136/bmj.315.7109.629

Eysenck, H. J. (1960). The concept of statistical significance and the controversy about one-tailed tests. Psychological Review, 67(4), 269-271. https://doi.org/10.1037/h0048412

Feather, S., \& Brier, R. (1968). The possible effect of the checker in precognition tests. Journal of Parapsychology, 32(3), 167-175.

Feather, S. R., \& Schmicker, M. (2005). The gift: The extraordinary paranormal experiences of ordinary people. London: Rider.

Ferguson, C. J., \& Heene, M. (2012). A vast graveyard of undead theories: Publication bias and psychological science's aversion to the null. Perspectives on Psychological Science, 7(6), 555-561. https://doi.org/10.1177/1745691612459059

Fiedler, K., Kutzner, F., \& Krueger, J. I. (2012). The long way from $\alpha$-error control to validity proper: Problems with a short-sighted false-positive debate. Perspectives on Psychological Science, 7(6), 661-669. https://doi.org/10.1177/1745691612462587 
Fisher, R. A. (1924). A method of scoring coincidences in tests with playing cards. In Proceedings of the Society for Psychical Research (Vol. 34, pp. 181-185). Glasgow, Scotland: Robert Maclehose \& Co.

Fisher, R. A. (1925). Statistical methods for research workers. Edinburgh, Scotland: Oliver \& Boyd.

Fitzpatrick, O., \& Shook, S. (1994). Belief in the paranormal: Does identity development during the college years make a difference? An initial investigation. Journal of Parapsychology, 58(3), 315-329.

Francis, G. (2012). Too good to be true: Publication bias in two prominent studies from experimental psychology. Psychonomic Bulletin \& Review, 19(2), 151156. https://doi.org/10.3758/s13423-012-0227-9

Franklin, M. S., Baumgart, S. L., \& Schooler, J. W. (2014). Future directions in precognition research: more research can bridge the gap between skeptics and proponents. Frontiers in Psychology, 5, 907. https://doi.org/10.3389/fpsyg.2014.00907

Freeman, J. (1966). Sex differences and atrget arrangement: High-school booklet tests of precognition. Journal of Parapsychology, 30(4), 227-235.

Freeman, J., \& Nielsen, W. (1964). Precognition score deviations as related to anxiety levels. Journal of Parapsychology, 28(4), 239-249.

French, C. (2003). Fantastic memories: The relevance of research into eyewitness testimony and false memories for reports of anomalous experiences. Journal of Consciousness Studies, 10(6-7), 153-174.

French, C. (2004). Parapsychology. In A. Kuper \& J. Kuper (Eds.), The social science encyclopedia (pp. 702-703). London: Routledge.

French, C. (2008). Is Parapsychology a Pseudoscience? In Conference on Science and Pseudoscience. Birmingham University. Retrieved from https://www.researchgate.net/publication/267772045_Is_Parapsychology_a_Ps eudoscience

French, C. (2012). Precognition studies and the curse of the failed replications. Retrieved from https://www.theguardian.com/science/2012/mar/15/precognition-studies-cursefailed-replications 
Galak, J., Leboeuf, R. A., Nelson, L. D., \& Simmons, J. P. (2012). Correcting the Past: Failures to Replicate Psi. Journal of Personality and Social Psychology, 103(6), 933-948. https://doi.org/10.1037/a0029709

Gatlin, L. L. (1977). Meaningful information creation: An alternative interpretation of the psi phenomenon. Journal of the American Society for Psychical Research, $71(1), 1-18$.

Gatlin, L. L. (1979). A new measure of bias in finite sequences with applications to ESP data. Journal of the American Society for Psychical Research, 73, 29-43.

Gelman, A. (2013). A new Bem theory. Retrieved from http://andrewgelman.com/2013/08/25/a-new-bem-theory/

Gelman, A. (2017). Daryl Bem and Arthur Conan Doyle. Retrieved from http://andrewgelman.com/2017/07/12/daryl-bem-arthur-conan-doyle/

Giles, D. C. (2002). Advanced research methods in psychology. London, England: Routledge. Retrieved from http://ebookcentral.proquest.com/lib/vuw/detail.action?docID=1144492

Gluckman, P. D., Hanson, M. A., \& Spencer, H. G. (2005). Predictive adaptive responses and human evolution. Trends in Ecology \& Evolution, 20(10), 527533. https://doi.org/10.1016/j.tree.2005.08.001

Goldberg, L. R. (1999). A broad-bandwidth, public domain, personality inventory measuring the lower-level facets of several five-factor models. Personality Psychology in Europe, 7(1), 7-28.

Goodfellow, L. D. (1938). A psychological interpretation of the results of the Zenith radio experiments in telepathy. Journal of Experimental Psychology, 23(6), 601-632. https://doi.org/10.1037/h0058392

Gosling, S. D., Rentfrow, P. J., \& Swann, W. B. (2003). A very brief measure of the Big-Five personality domains. Journal of Research in Personality, 37(6), 504528. https://doi.org/10.1016/S0092-6566(03)00046-1

Greeley, A. (1987). The 'impossible': It's happening. Noetic Sciences Review, 2, 7-9.

Green, C. E. (1960). Analysis of spontaneous cases. In Proceedings of the Society for Psychical Research (Vol. 53, pp. 97-161). Glasgow, Scotland: Robert Maclehose \& Co.

Greenwald, A. G., Mcghee, D. E., \& Schwartz, J. L. K. (1998). Measuring individual differences in implicit cognition: The implicit association test. Journal of 
Personality and Social Psychology, 74(6), 1464-1480.

https://doi.org/10.1037/0022-3514.74.6.1464

Greville, T. N. E. (1944). On multiple matching with one variable deck. The Annals of Mathematical Statistics, 15(4), 432-434.

https://doi.org/10.1214/aoms/1177731215

Gutierrez, L., \& Bourgon, G. (2012). Drug treatment courts: A quantitative review of study and treatment quality. Justice Research and Policy, 14(2), 47-77. https://doi.org/10.3818/JRP.14.2.2012.47

Halpern, A., \& Devereaux, S. (1989). Lucky numbers: Choice strategies in the Pennsylvania Daily Number game. Bulletin of the Psychonomic Society, 27(2), 167-170. https://doi.org/10.3758/BF03329930

Hansel, C. E. M. (1966). ESP: A scientific evaluation. New York City, NY: Charles Scribner's Sons.

Hansel, C. E. M. (1980). ESP and parapsychology: A critical re-evaluation. Buffalo, NY: Prometheus Books.

Haraldsson, E. (1975). Reported dream recall, precognitive dreams, and ESP. In J. D. Morris, W. G. Roll, \& R. L. Morris (Eds.), Research in parapsychology 1974 (pp. 47-48). Metuchen, NJ: Scarecrow Press.

Haraldsson, E. (1978). ESP and the defense mechanism test (DMT): A further validation. European Journal of Parapsychology, 2, 104-114.

Haraldsson, E. (1980). Scoring in a precognition test as a function of the frequency of reading on psychical phenomena and belief in ESP. Research Letter of the Parapsychological Division of the Psychological Laboratory of the University of Utrecht, 1, 1-8.

Haraldsson, E. (1993). Are religiosity and belief in an afterlife better predictors of ESP performance than belief in psychic phenomena? Journal of Parapsychology, 57(3), 259-273.

Haraldsson, E., Houtkooper, J. M., Schneider, R., \& Bäckström, M. (2002). Perceptual defensiveness and ESP performance: Reconstructed DMT ratings and psychological correlates in the first German DMT-ESP experiment. Journal Of Parapsychology, 66(3), 249-270.

Haraldsson, E., \& Johnson, M. (1979). ESP and the defence mechanism test (DMT), Icelandic study no. III.: A case of experimenter effect? European Journal of Parapsychology, 3, 11-20. 
Haraldsson, E., \& Johnson, M. (1986). The Defense Mechanism Test (DMT) as a predictor of ESP performance: Icelandic studies VI and VII. In D. H. Weiner \& D. I. Radin (Eds.), Research in Parapsychology 1985 (pp. 43-44). Metuchen, NJ: Scarecrow Press.

Hedges, L. V. (1984). Estimation of effect size under nonrandom sampling: The effects of censoring studies yielding statistically insignificant mean differences. Journal of Educational Statistics, 9(1), 61-85. https://doi.org/10.2307/1164832

Hemmings, S., \& Irwin, H. J. (1993). Tendency toward controlling behavior in adult children of problem drinkers: An experimental study. Psychological Reports, 72(2), 691-700. https://doi.org/10.2466/pr0.1993.72.2.691

Hines, T. (2003). Pseudoscience and the paranormal (2nd ed.). Amherst, NY: Prometheus Books.

Hitchman, G. A. M., Roe, C. A., \& Sherwood, S. J. (2012). A reexamination of nonintentional precognition with openness to experience, creativity, psi beliefs, and luck beliefs as predictors of success. Journal of Parapsychology, 76(1), $109-145$.

Holt, N. J. (2002). Creativity and boundary permeability. Unpublished manuscript, University of Northampton, UK.

Honorton, C. (1967). Creativity and precognition scoring level. Journal of Parapsychology, 31(1), 29-42.

Honorton, C. (1972). Reported frequency of dream recall and ESP. Journal of the American Society for Psychical Research, 66(4), 369-374.

Honorton, C. (1985). Meta-analysis of psi ganzfeld research: A response to Hyman. Journal of Parapsychology, 49(1), 51-91.

Honorton, C. (1987). Precognition and real-time ESP performance in a computer task with an exceptional subject. Journal of Parapsychology, 51(4), 291-320.

Honorton, C., \& Ferrari, D. (1989). 'Future telling': A meta-analysis of forced-choice precognition experiments, 1935-1987. Journal of Parapsychology, 53(4), 281308.

Honorton, C., Ferrari, D., \& Bem, D. (1998). Extraversion and ESP performance: A meta-analysis and a confirmation. Journal of Parapsychology, 62(3), 255-276.

Honorton, C., \& Harper, S. (1974). Psi-mediated imagery and ideation in an experimental procedure for regulating perceptual input. Journal of the American Society for Psychical Research, 68, 156-168. 
Houran, J., \& Lange, R. (1998). Modeling precognitive dreams as meaningful coincidences. Psychological Reports, 83(3 Suppl), 1411-1414. https://doi.org/10.2466/pr0.1998.83.3f.1411

Houran, J., Lange, R., \& Hooper, D. (2018). Cross-examining the case for precognition: Comment on Mossbridge and Radin (2018). Psychology of Consciousness: Theory, Research, and Practice, 5(1), 98-109. https://doi.org/10.1037/cns0000126

Huettel, S. A., Mack, P. B., \& Mccarthy, G. (2002). Perceiving patterns in random series: dynamic processing of sequence in prefrontal cortex. Nature Neuroscience, 5(5), 485-490. https://doi.org/10.1038/nn841

Humphrey, B. (1945). An exploratory correlation study of personality measures and ESP scores. Journal of Parapsychology, 9(2), 116-123.

Hunter, R. N., \& Derr, J. S. (1978). Prediction monitoring and evaluation program; a progress report. Earthquake Information Bulletin, 10(3), 93-96.

Hyman, R. (1964). The nature of psychological inquiry. Eaglewood Cliffs, NJ: Prentice Hall.

Hyman, R. (1977). The case against parapsychology. The Humanist, 37, 37-49.

Hyman, R. (1985). The ganzfeld psi experiment: A critical appraisal. Journal of Parapsychology, 49(1), 3-49.

Hyman, R. (1994). Anomaly or artifact? Comments on Bem and Honorton. Psychological Bulletin, 115(1), 19-24. https://doi.org/10.1037/00332909.115.1.19

Hyman, R. (1995). Evaluation of the program on anomalous mental phenomena. Journal of Parapsychology, 59(4), 321-351.

Hyman, R. (2010a). Meta-analysis that conceals more than it reveals: Comment on Storm et al. (2010). Psychological Bulletin, 136(4), 486-490. https://doi.org/10.1037/a0019676

Hyman, R. (2010b). Parapsychology's achilles heel: Persistent inconsistency. In S. Krippner \& H. L. Friedman (Eds.), Debating psychic experience (pp. 43-52). Oxford: Praeger.

Hyman, R. (2017). Pitfalls in parapsychological research. In S. O. Lilienfeld \& I. D. Waldman (Eds.), Psychological science under scrutiny (pp. 271-294). Malden, MA: Wiley-Blackwell. Retrieved from https://onlinelibrary.wiley.com/doi/10.1002/9781119095910.ch14 
Ioannidis, J. P. A. (2005). Why most published research findings are false. CHANCE, 18(4), 40-47. https://doi.org/10.1080/09332480.2005.10722754

Irwin, H. J. (1989). On paranormal belief: The psychology of the sceptic. In J. F. Zollschan, J. F. Schumaker, \& G. F. Walsh (Eds.), Exploring the paranormal: Perspectives on belief and experience (pp. 305-312). Bridport: Prism Press.

Irwin, H. J. (2009). The psychology of paranormal belief: A researcher's handbook. Hatfield, Hertfordshire: University Of Hertfordshire Press.

Irwin, H. J., \& Watt, C. A. (2007). An introduction to parapsychology (5th ed.). Jefferson, NC: McFarland.

John, L. K., Loewenstein, G., \& Prelec, D. (2012). Measuring the prevalence of questionable research practices with incentives for truth telling. Psychological Science, 23(5), 524-532. https://doi.org/10.1177/0956797611430953

Johnson, M. (1969). Attitude and target differences in a group precognition test. Journal of Parapsychology, 33, 324-325.

Johnson, M., \& Haraldsson, E. (1984). The Defense Mechanism Test as a predictor of ESP scores: Icelandic studies IV and V. Journal of Parapsychology, 48(3), $185-200$.

Judd, C. M., \& Gawronski, B. (2011). Editorial comment. Journal of Personality and Social Psychology, 100(3), 406. https://doi.org/10.1037/0022789

Jung, C. (1973). Synchronicity: An acausal connecting principle. Princeton, NJ: Princeton/Bollingen.

Kahneman, D. (2013). Thinking, fast and slow (1st ed.). New York: Farrar, Straus and Giroux.

Kanthamani, H., \& Rao, H. (1975). Response tendencies and stimulus structure. Journal of Parapsychology, 39(2), 97-105.

Kennedy, J. E. (2001). Why is PSI so elusive? A review and proposed model. Journal of Parapsychology, 65(3), 219-245.

Kennedy, J. E. (2004). A proposal and challenge for proponents and skeptics of psi. Journal of Parapsychology, 68(1), 157-167.

Kennedy, J. E. (2015). Beware of inferential errors and low power with Bayesian analyses: Power analysis is needed for confirmatory research. Journal of Parapsychology, 79(1), 53-64.

Kennedy, J. E. (2016). Is the methodological revolution in psychology over or just beginning? Journal of Parapsychology, 80(2), 156-168. 
Klauer, K. C., \& Musch, J. (2003). Affective priming: Findings and theories. In J. Musch \& K. C. Klauer (Eds.), The psychology of evaluation: Affective processes in cognition and emotion (pp. 7-49). Mahwah, NJ: Erlbaum.

Knapp, R. H., \& Garbutt, J. T. (1958). Time imagery and the achievement motive. Journal of Personality, 26(3), 426-434. https://doi.org/10.1111/j.14676494.1958.tb01597.x

Koerner, B. (2017). Russians engineer a brilliant slot machine cheat — and casinos have no fix. Retrieved from https://www.wired.com/2017/02/russians-engineerbrilliant-slot-machine-cheat-casinos-no-fix/

Kragh, U., \& Smith, G. (Eds.). (1970). Percept-genetic analysis. Lund, Sweden: Gleerup.

Lakens, D. (2015). Why a meta-analysis of 90 precognition studies does not provide convincing evidence of a true effect. Retrieved from http://daniellakens.blogspot.co.nz/2015/04/why-meta-analysis-of-90precognition.html

Lang, P. J., \& Greenwald, M. K. (1993). International Affective Picture System standardization procedure and results for affective judgments. Gainesville: University of Florida Center for Research in Psychophysiology.

Lange, R., Thalbourne, M. A., Houran, J., \& Storm, L. (2000). The revised transliminality scale: Reliability and validity data from a rasch top-down purification procedure. Consciousness and Cognition, 9(4), 591-617. https://doi.org/10.1006/cog.2000.0472

Lattal, K. A. (1998). A century of effect: Legacies of E. L. Thorndike's animal intelligence monograph. Journal of the Experimental Analysis of Behavior, 70(3), 325-336. https://doi.org/10.1901/jeab.1998.70-325

Lawrence, T. R. (1990-1991). Subjective random generations and the reversed sheepgoat effect: A failure to replicate. European Journal of Parapsychology, 8 , $131-144$.

Lawrence, T. R. (1993). Gathering in the sheep and goats: A meta-analysis of forcedchoice sheep-goat ESP studies, 1947-1993. In Proceedings of Presented Papers: The Parapsychological Association 36th Annual Convention (pp. 7586).

Lawrence, T. R., Edwards, C., Barraclough, N., Church, S., \& Hetherington, F. (1995). Modelling childhood causes of paranormal belief and experience: Childhood 
trauma and childhood fantasy. Personality and Individual Differences, 19(2), 209-215. https://doi.org/10.1016/0191-8869(95)00034-4

L'Ecuyer, P. (2001). Software for uniform random number generation: distinguishing the good and the bad. In Proceedings of the Winter Simulation Conference (Vol. 1, pp. 95-105). USA: IEEE. https://doi.org/10.1109/WSC.2001.977250

Liberati, A., Altman, D. G., Tetzlaff, J., Mulrow, C., Gøtzsche, P. C., Ioannidis, J. P. A., ... Moher, D. (2009). The PRISMA statement for reporting systematic reviews and meta-analyses of studies that evaluate healthcare interventions: explanation and elaboration. British Medical Journal, 339(jul21 1), b2700. https://doi.org/10.1136/bmj.b2700

Libet, B., Gleason, C. A., Wright, E. W., \& Pearl, D. K. (1983). Time of conscious intention to act in relation to onset of cerebral activity (readiness-potential): The unconscious initiation of a freely voluntary act. Brain, 106(3), 623-642. https://doi.org/10.1093/brain/106.3.623

Lotto New Zealand. (n.d.). Retrieved from https://mylotto.co.nz/

Lovitts, B. (1981). The Sheep-goat effect turned upside down. Journal of Parapsychology, 45(4), 293-309.

Luke, D., Delanoy, D., \& Sherwood, S. J. (2003). Questionnaire of beliefs about luck. Unpublished instrument, University of Northampton, UK.

Luke, D., Delanoy, D., \& Sherwood, S. J. (2008). Psi may look like luck: Perceived luckiness and beliefs about luck in relation to precognition. Journal of the Society for Psychical Research, 72(4), 193-207.

Luke, D., \& Morin, S. (2009). Luck beliefs, PMIR, psi and the sheep-goat effect: A replication. In Abstracts of papers presented at the 33rd International Conference of the Society for Psychical Research (pp. 12-13). University of Nottingham: Society for Psychical Research.

Luke, D., \& Morin, S. (2014). Exploration of the validity and utility of a reward contingency in a non-intentional forced-choice precognition task. Journal of the Society for Psychical Research, 78(4), 207-218.

Luke, D., Roe, C., \& Davison, J. (2008). Testing for forced-choice precognition using a hidden task: Two replications. Journal of Parapsychology, 72, 133-154.

Luke, D., \& Zychowicz, K. (2014). Comparison of outcomes with nonintentional and intentional precognition tasks. Journal of Parapsychology, 78(2), 223-234. 
Lund, F. H. (1939). Extra-sensory-perception another name for free association? Journal of General Psychology, 20(1), 235-238. https://doi.org/10.1080/00221309.1939.9710005

Lyons, L. (2005). Paranormal beliefs come (super)naturally to some. Retrieved from http://news.gallup.com/poll/19558/Paranormal-Beliefs-Come-SuperNaturallySome.aspx

Mangan, G. L. (1955). Evidence of displacement in a precognition test. Journal of Parapsychology, 19(1), 35-44.

Mangan, G. L. (1957). An ESP experiment with dual-aspect targets involving one trial a day. Journal of Parapsychology, 21(4), 273-283.

Mangan, G. L. (1958). A review of published research on the relationship of some personality variables to ESP scoring level. New York, NY: Parapsychology Foundation.

Martin, D. J., Abramson, L. Y., \& Alloy, L. B. (1984). Illusion of control for self and others in depressed and nondepressed college students. Journal of Personality and Social Psychology, 46(1), 125-136. https://doi.org/10.1037/00223514.46 .1 .125

Marwaha, S. B., \& May, E. C. (2015). The multiphasic model of precognition: the rationale. Journal of Parapsychology, 79(1), 5-19.

May, E. C., \& Depp, J. G. (2015). Entropy and precognition: The physics domain of the Multiphasic Model of Precognition. In E. C. May \& S. B. Marwaha (Eds.), Extransensory perception: Support, skepticism, and science (Vol. 2). Santa Barbara, CA: ABC-CLIO.

McCarthy, D., \& Schechter, E. I. (1986). Estimating effect size from critical ratios. In D. H. Weiner \& D. I. Radin (Eds.), Research in parapsychology 1985 (pp. 9596). Metuchen, NJ: Scarecrow Press.

McClenon, J. (1982). Two experiments in the practical application of psi [Abstract]. Journal of Parapsychology, 46, 59.

McCrae, R. R., \& Costa, P. T. (1987). Validation of the five-factor model of personality across instruments and observers. Journal of Personality and Social Psychology, 52(1), 81-90. https://doi.org/10.1037/0022-3514.52.1.81

Meier-Civelli, U., \& Stoll, F. (1990). An evaluative study of the defense mechanism test. US Army Research Institute for the Behavioral and Social Sciences. 
Merriam-Webster. (2018). Paranormal. Retrieved from https://www.merriamwebster.com/dictionary/paranormal

Milton, J. (1999). Should ganzfeld research continue to be crucial in the search for a replicable psi effect? Part I. Discussion paper and introduction to an electronicmail discussion. Journal of Parapsychology, 63(4), 309-333.

Milton, J., \& Wiseman, R. (1999a). A meta - analysis of mass - media tests of extrasensory perception. British Journal of Psychology, 90(2), 235-240. https://doi.org/10.1348/000712699161378

Milton, J., \& Wiseman, R. (1999b). Does psi exist? Lack of replication of an anomalous process of information transfer. Psychological Bulletin, 125(4), 387-391. https://doi.org/10.1037/0033-2909.125.4.387

Morris, R. L. (1980). Psi functioning within a simple communication model. In B. Shapin \& L. Coly (Eds.), Communication and parapsychology (pp. 1-24). New York: Parapsychology Foundation.

Mossbridge, J., \& Radin, D. (2018). Plausibility, statistical interpretations, physical mechanisms and a new outlook: Response to commentaries on a precognition review. Pyschology of Consciousness: Theory, Research, and Practice, 5(1), $110-116$.

Mossbridge, J., Tressoldi, P., \& Utts, J. (2012). Predictive physiological anticipation preceding seemingly unpredictable stimuli: a meta-analysis. Frontiers in Psychology, 3, 390. https://doi.org/10.3389/fpsyg.2012.00390

Mousseau, M.-C. (2003). Parapsychology: Science or pseudo-science. Journal of Scientific Exploration, 17(2), 271-282.

Mundle, C. W. K. (1964). Does the concept of precognition make sense? International Journal of Parapsychology, 6, 179-198.

Murray, A. L. (2011). The validity of the meta-analytic method in addressing the issue of psi replicability. Journal of Parapsychology, 75(2), 261-277.

Nash, C. B. (1964). A television test on ESP. International Journal of Parapsychology, $6,139-142$.

Nazimuddin, S. K. (2015). A Study of Individual Differences in Educational Situations. International Journal of Scientific Engineering and Research, 3, $180-184$. 
Neuberg, S. L., Kenrick, D. T., \& Schaller, M. (2011). Human threat management systems: Self-protection and disease avoidance. Neuroscience and Biobehavioral Reviews, 35(4), 1042-1051. https://doi.org/10.1016/j.neubiorev.2010.08.011

NZ Lotteries Commission. (n.d.). Game odds. Retrieved from https://assets.mylotto.co.nz/assets/uploads/cc7ac4b6-1049-11e7-b94b46046049c92f.pdf

O’Brien, J. T. (1979). An examination of the checker effect. In W. G. Roll (Ed.), Research in Parapsychology 1978 (pp. 153-155). Metuchen, NJ: Scarecrow Press.

Open Science Collaboration, Nosek, B. A., Aarts, A. A., Anderson, C. J., Anderson, J. E., \& Kappes, H. B. (2015). Estimating the reproducibility of psychological science. Science, 349, aaac4716-1-aaac4716-8. https://doi.org/10.1126/science.aac4716

Orme, J. E. (1974). Precognition and time. Journal of the Society for Psychical Research, 47, 351-365.

Osherovich, L. (2011). Hedging against academic risk. Science-Business EXchange, 4(15), 416. https://doi.org/10.1038/scibx.2011.416

Otis, L. P., \& Alcock, J. (1982). Factors affecting extraordinary belief. Journal of Social Psychology, 118(1), 77-85. https://doi.org/10.1080/00224545.1982.9924420

Palmer, J. (1972). Scoring in ESP tests as a function of belief in ESP. Part II. Beyond the Sheep-goat effect. Journal of the American Society for Psychical Research, $66(1), 1-26$.

Palmer, J. (1978). Extrasensory perception: Research findings. In S Krippner (Ed.), Advances in parapsychological research 2 (pp. 59-243). New York, NY: Springer.

Palmer, J. (2003). ESP in the ganzfeld: Analysis of a debate. Journal of Consciousness Studies, 10(F0020006), 51-68.

Palmer, J. (2016). Hansel's ghost: Resurrection of the experimenter fraud hypothesis in parapsychology. Journal of Parapsychology, 80(1), 5-16.

Parker, A., Grams, D., \& Pettersson, C. (1998). Further variables relating to psi in the ganzfeld. Journal of Parapsychology, 62(4), 319-337. 
Parker, A., \& Millar, B. (2014). Revealing psi secrets: Successful experimenters seem to succeed by using their own psi. Journal of Parapsychology, 78(1), 39-55.

Parker, A., \& Sjödén, B. (2010). Do some of us habituate to future emotional events? Journal of Parapsychology, 74(1), 99-115.

Peltzer, K. (2003). Magical thinking and paranormal beliefs among secondary and university students in South Africa. Personality and Individual Differences, 35(6), 1419-1426. https://doi.org/10.1016/S0191-8869(02)00359-8

Perkins, S. L., \& Allen, R. (2006). Childhood physical abuse and differential development of paranormal belief systems. The Journal of Nervous and Mental Disease, 194(5), 349-355. https://doi.org/10.1097/01.nmd.0000217832.85665.c5

Persinger, M. A., \& Makarec, K. (1987). Temporal lobe epileptic signs and correlative behaviors displayed by normal populations. Journal of General Psychology, 114(2), 179-195. https://doi.org/10.1080/00221309.1987.9711068

Pew Research Center. (2009). Many Americans mix multiple faiths. Retrieved from http://www.pewforum.org/2009/12/09/many-americans-mix-multiple-faiths/

Pratt, J. G., Smith, B. M., Rhine, J. B., Stuart, C. E., \& Greenwood, J. A. (1940). Extra-sensory perception after sixty years: A critical appraisal of the research in extra-sensory perception. New York, NY: Henry Holt. Retrieved from http://psycnet.apa.org/psycinfo/2008-17044-000

Prinz, F., Schlange, T., \& Asadullah, K. (2011). Believe it or not: How much can we rely on published data on potential drug targets? Nature Reviews Drug Discovery, 10(9), 712-713. https://doi.org/10.1038/nrd3439-c1

Pyszczynski, T., Greenberg, J., \& Solomon, S. (2000). Proximal and distal defense: A new perspective on unconscious motivation. Current Directions in Psychological Science, 9(5), 156-160. https://doi.org/10.1111/14678721.00083

Radin, D. I. (1997). Unconscious perception of future emotions: An experiment in presentiment. Journal of Scientific Exploration, 11(2), 163-180.

Radin, D. I. (2004). Event-related electroencephalographic correlations between isolated human subjects. Journal of Alternative and Complementary Medicine, 10(2), 315-323. https://doi.org/10.1089/107555304323062301

Radin, D. I. (2006). Entangled minds: Extrasensory experiences in a quantum reality. New York, NY: Paraview Pocket Books. 
Randi, J. (1964). The million dollar challenge. Retrieved from http://web.randi.org/themillion-dollar-challenge.html

Rao, K. (1963). Studies in the preferential effect II. A language ESP test involving precognition and 'intervention'. Journal of Parapsychology, 27(3), 147-160.

Reader's Digest Magazine. (2006). Britons report 'psychic powers'. Retrieved from http://news.bbc.co.uk/2/hi/uk_news/5017910.stm

Reber, A. S. (1967). Implicit learning of artificial grammars. Journal of Verbal Learning and Verbal Behavior, 6(6), 855-863. https://doi.org/10.1016/S00225371(67)80149-X

Reiser, M., Ludwig, L., Saxe, S., \& Wagner, C. (1979). Evaluation of the use of psychics in the investigation of major crimes. Journal of Police Science and Administration, 7(1), 18-25.

Revoir, P. (2009). OK Derren, now tell us how you REALLY did it: Experts pour scorn on illusionist's explanation of lottery stunt. Retrieved from http://www.dailymail.co.uk/tvshowbiz/article-1212839/Okay-Derren-Browntell-REALLY-did-Experts-pour-scorn-illusionists-explanation-lotterystunt.html

Rhine, J. B. (1934). Extra-sensory perception. Boston: Boston Society for Psychic Research.

Rhine, J. B. (1938). Experiments bearing on the precognition hypothesis. Journal of Parapsychology, 2(1), 38-54.

Rhine, J. B. (1941). Experiments bearing upon the precognition hypothesis. III. Mechanically selected cards. Journal of Parapsychology, 5(1), 1-58.

Rhine, J. B. (1977). History of experimental studies. In B. B. Wolfman (Ed.), Handbook of parapsychology (pp. 25-47). New York: Van Nostrand Reinhold.

Rhine, L. E. (1954). Frequency of types of experience in spontaneous precognition. Journal of Parapsychology, 18(2), 93-123.

Rhine, L. E. (1955). Precognition and intervention. Journal of Parapsychology, 19(1), $1-34$.

Richet, C. (1884). La suggestion mentale et le calcul des probabilités. Revue Philosophique de la France et de l'Étranger, 18, 609-674.

Ritchie, S. J., Wiseman, R., \& French, C. C. (2012). Failing the future: Three unsuccessful attempts to replicate Bem's 'retroactive facilitation of recall' effect. PLoS ONE, 7(3), e33423. https://doi.org/10.1371/journal.pone.0033423 
Roe, C. A. (2017). Has parapsychology made progress? Mindfield, 9(2), 42-47.

Roe, C. A., Henderson, S. J., \& Matthews, J. (2008). Extraversion and performance at a forced-choice ESP task with verbal stimuli: Two studies. Journal of the Society for Psychical Research, 72(893), 208-220.

Roese, N. J., \& Vohs, K. D. (2012). Hindsight Bias. Perspectives on Psychological Science, 7(5), 411-426. https://doi.org/10.1177/1745691612454303

Rogers, D., \& Carpenter, J. (1966). The decline of variance of ESP scores within a testing session. Journal of Parapsychology, 30(3), 141-150.

Rosenthal, R. (1979). The 'file drawer problem' and tolerance for null results. Psychological Bulletin, 86(3), 638-641. https://doi.org/10.1037/00332909.86.3.638

Rosenthal, R. (1986). Meta-analytic procedures and the nature of replication: The ganzfeld debate. Journal of Parapsychology, 50(4), 315-336.

Ross, C. A., \& Joshi, S. (1992). Paranormal experiences in the general population. The Journal of Nervous and Mental Disease, 180(6), 357-361.

Rothstein, H. (2008). Publication bias as a threat to the validity of meta-analytic results. Journal of Experimental Criminology, 4(1), 61-81. https://doi.org/10.1007/s11292-007-9046-9

Rouder, J., \& Morey, R. (2011). A Bayes factor meta-analysis of Bem's ESP claim. Psychonomic Bulletin \& Review, 18(4), 682-689. https://doi.org/10.3758/s13423-011-0088-7

Rudski, J. (2004). The illusion of control, superstitious belief, and optimism. Current Psychology, 22(4), 306-315. https://doi.org/10.1007/s12144-004-1036-8

Sannwald, G. (1963). On the psychology of spontaneous paranormal phenomena. International Journal of Parapsychology, 5(3), 274-292.

Sartori, L., Massacessi, S., Martinelli, M., \& Tressoldi, P. E. (2004). Physiological correlates of ESP: heart rate differences between targets and nontargets. Journal of Parapsychology, 68(2), 351-360.

Schimmack, U. (2012). The ironic effect of significant results on the credibility of multiple-study articles. Psychological Methods, 17(4), 551-566. https://doi.org/10.1037/a0029487

Schlitz, M., Wiseman, R., Watt, C., \& Radin, D. I. (2006). Of two minds: Scepticproponent collaboration within parapsychology. British Journal of Psychology, 97(3), 313-322. https://doi.org/10.1348/000712605X80704 
Schmeidler, G. (1943). Predicting good and bad scores in a clairvoyance experiment: A preliminary report. Journal of the American Society for Psychical Research, $37,103-110$.

Schmeidler, G. (1945). Separating the Sheep from the Goats. Journal of the American Society for Psychical Research, 39, 47-49.

Schmeidler, G. (1964a). An experiment on precognitive clairvoyance: Part I. The Main Results. Journal of Parapsychology, 28(1), 1-14.

Schmeidler, G. (1964b). An experiment on precognitive clairvoyance: Part III. Precognition scores related to the subjects' ways of viewing time. Journal of Parapsychology, 28(2), 94-101.

Schmeidler, G. (1964c). An experiment on precognitive clairvoyance: Part IV. Precognition scores related to creativity. Journal of Parapsychology, 28(2), $102-108$.

Schmeidler, G. (1964d). An experiment on precognitive clairvoyance: Part V. Precognition scores related to feelings of success. Journal of Parapsychology, $28(2), 109-125$.

Schmeidler, G. (1971). Parapsychologists' opinions about parapsychology. Journal of Parapsychology, 35(3), 208-218.

Schmidt, H. (1969). Precognition of a quantum process. Journal of Parapsychology, 33(2), 99-108.

Schmidt, S., Schneider, R., Utts, J., \& Walach, H. (2004). Distant intentionality and the feeling of being stared at: Two meta-analyses. British Journal of Psychology, 95, 235-247. https://doi.org/10.1348/000712604773952449

Schoenberg, F. P. (2016). Introduction to probability with Texas Hold 'em examples (2nd ed.). Boca Raton: Chapman and Hall/CRC.

Schouten, S. (1993). Are we making progress. In L. Coly \& J. McMahon (Eds.), Psi research methodology: A re-examination, proceedings of an international conference (pp. 295-328). New York: Parapsychology Foundation.

Schultze-Kraft, M., Birman, D., Rusconi, M., Allefeld, C., Görgen, K., Dähne, S., ... Haynes, J.-D. (2016). The point of no return in vetoing self-initiated movements. Proceedings of the National Academy of Sciences, 113(4), 10801085. https://doi.org/10.1073/pnas.1513569112

Schumaker, J. F. (1990). Wings of illusion: The origin, nature, and future of paranormal belief. Buffalo, NY: Prometheus Books. 
Schwartz, S. A., \& De Mattei, R. J. (1983). The Mobius Psi-Q test: Preliminary findings. In R. A. White \& J. Solfvin (Eds.), Research in Parapsychology 1982 (pp. 103-105). Metuchen, NJ: Scarecrow Press.

Sheldrake, R. (1999). How widely is blind assessment used in scientific research? Alternative Therapies in Health and Medicine, 5(3), 88-91.

Shermer, M. (n.d.). The demographics of belief. Retrieved from https://michaelshermer.com/the-believing-brain/excerpt/

Silver, N., Mcculley, W., Chambliss, L., Charles, C., Smith, A., Waddell, W., \& Winfield, E. (1988). Sex and racial differences in color and number preferences. Perceptual and Motor Skills, 66, 295-299. https://doi.org/10.2466/pms.1988.66.1.295

Simmonds, M. (2015). Quantifying the risk of error when interpreting funnel plots. Systematic Reviews, 4, 24. https://doi.org/10.1186/s13643-015-0004-8

Simmons, J. P., Nelson, L. D., \& Simonsohn, U. (2011). False-positive psychology: Undisclosed flexibility in data collection and analysis allows presenting anything as significant. Psychological Science, 22(11), 1359-1366. https://doi.org/10.1177/0956797611417632

Simon, W. E. (1971). Number and color responses of some college students: Preliminary evidence for a "Blue Seven Phenomenon". Perceptual and Motor Skills, 33(2), 373-374. https://doi.org/10.2466/pms.1971.33.2.373

Simonsohn, U., Nelson, L. D., \& Simmons, J. P. (2014). P-curve: A key to the filedrawer. Journal of Experimental Psychology: General, 143(2), 534-547. https://doi.org/10.1037/a0033242

Sjöberg, L., \& Wåhlberg, A. A. (2002). Risk perception and new age beliefs. Risk Analysis, 22(4), 751-764. https://doi.org/10.1111/0272-4332.00066

Smith, J. C. (2009). Pseudoscience and extraordinary claims of the paranormal: A critical thinker's toolkit. Malden, MA: Wiley-Blackwell.

Smith, M. D. (1998). Perception of one's own luck: The formation, maintenance and consequences of perceived luckiness. Unpublished manuscript, University of Hertfordshire, UK.

Smith, M. D. (2003). The role of the experimenter in parapsychological research. Journal of Consciousness Studies, 10(6-7), 69-84. 
Smith, M. D., Wiseman, R., Machin, D., Harris, P., \& Joiner, R. (1997). Luckiness, competition, and performance on a psi task. Journal of Parapsychology, 61(1), $33-43$.

Soal, S. G., \& Goldney, K. M. (1943). Experiments in precognitive telepathy. In Proceedings of the Society for Psychical Research (Vol. 47, pp. 21-150). Glasgow, Scotland: Robert Maclehose \& Co.

Sondow, N. (1988). The decline of precognized events with the passage of time: Evidence from spontaneous dreams. Journal of the American Society for Psychical Research, 82(1), 33-51.

Stanford, R. G. (1970). Extrasensory effects upon memory. Journal of the American Society for Psychical Research, 64(2), 161-186.

Stanford, R. G. (1973). Extrasensory effects upon associative processes in a directed free-response task. Journal of the American Society for Psychical Research, 67(2), 147-190.

Stanford, R. G. (1974). An experimentally testable model for spontaneous psi events: I. Extrasensory events. Journal of the American Society for Psychical Research, $68,34-57$.

Stanford, R. G. (1977). Conceptual frameworks of contemporary psi research. In B. B. Wolfman (Ed.), Handbook of parapsychology (pp. 823-858). London, England: Van Nostrand Reinhold.

Stanford, R. G. (1982). An experimentally testable model for spontaneous extrasensory events. In I. Grattan-Guiness (Ed.), Psychical research: A guide to its history, principles and practices (pp. 195-205). Wellingborough, UK: Aquarian Press.

Stanford, R. G. (1990). An experimentally testable model for spontaneous psi events: A review of related evidence and concepts from parapsychology and other sciences. In S. Krippner (Ed.), Advances in parapsychological research 6 (pp. 54-161). Jefferson, NC: McFarland.

Steinkamp, F. (2005). Forced-choice ESP experiments: Their past and their future. In M. A. Thalbourne \& L. Storm (Eds.), Parapsychology in the 21st century: Essays on the future of psychical research (pp. 124-163). Jefferson, NC: McFarland.

Steinkamp, F., Milton, J., \& Morris, R. (1998). A meta-analysis of forced-choice experiments comparing clairvoyance and precognition. Journal of Parapsychology, 62(3), 193-218. 
Sterling, T. D. (1959). Publication decisions and their possible effects on inferences drawn from tests of significance - or vice versa. Journal of the American Statistical Association, 54(285), 30-34. https://doi.org/10.1080/01621459.1959.10501497

Sterling, T. D., Rosenbaum, W. L., \& Weinkam, J. J. (1995). Publication decisions revisited: The effect of the outcome of statistical tests on the decision to publish and vice versa. The American Statistician, 49(1), 108-112. https://doi.org/10.1080/00031305.1995.10476125

Storm, L. (2002). A parapsychological investigation of the I Ching: Seeking Psi in an ancient Chinese system of divination. Australian Journal of Parapsychology, 2(1), 44-62.

Storm, L. (2006a). A parapsychological investigation of the I Ching: The relationship between psi, intuition, and time perspective. Journal of Parapsychology, 70(1), $121-141$.

Storm, L. (2006b). Meta-Analysis in Parapsychology: I. The Ganzfeld Domain. Australian Journal of Parapsychology, 6(1), 35-53.

Storm, L. (2008). Investigations of the I Ching: I. Relationships between psi and time perspective, paranormal belief and meaningfulness. Australian Journal of Parapsychology, 8(2), 103-127.

Storm, L. (2016). The Sheep-Goat effect. Retrieved from https://psiencyclopedia.spr.ac.uk/articles/sheep-goat-effect

Storm, L., \& Thalbourne, M. A. (1998-1999). The transliminal connection between paranormal effects and personality in an experiment with the I Ching. European Journal of Parapsychology, 14, 100-124.

Storm, L., \& Thalbourne, M. A. (2001). Studies of the I Ching: I. A replication. Journal of Parapsychology, 65(2), 105-124.

Storm, L., \& Tressoldi, P. E. (2017). Gathering in more sheep and goats: A metaanalysis of forced-choice sheep-goat ESP studies, 1994-2015. Journal of the Society for Psychical Research, 81(2), 79-107.

Storm, L., Tressoldi, P. E., \& di Risio, L. (2012). Meta-analysis of ESP studies, 19872010: Assessing the success of the forced-choice design in parapsychology. Journal of Parapsychology, 76(2), 243-273. 
Stowell, M. S. (1997). Precognitive dreams: A phenomenological study. Part II. Discussion. Journal of the American Society for Psychical Research, 91(4), 255-304.

Stradling, G. F. (1907). A résumé of the literature of the N rays, the N1 rays, the physiological rays and the heavy emission, with a bibliography. Journal of the Franklin Institute, 164(6), 57-74, 113-130, 177-189. https://doi.org/10.1016/S0016-0032(07)90056-3

Targ, R., \& Puthoff, H. (1974). Information transmission under conditions of sensory shielding. Nature, 251(5476), 602-607. https://doi.org/10.1038/251602a0

Tart, C. (1983). Information acquisition rates in forced-choice ESP experiments: Precognition does not work as well as present-time ESP. Journal of the American Society for Psychical Research, 77, 293-310.

Tart, C. (2002). Parapsychology and transpersonal psychology: 'Anomalies' to be explained away or spirit to manifest? Journal of Parapsychology, 66(1), 31-47.

Tart, C., Puthoff, H., \& Targ, R. (1980). Information transmission in remote viewing experiments. Nature, 284(5752), 191. https://doi.org/10.1038/284191a0

Taves, E., \& Dale, L. A. (1943). The Midas Touch in psychical research. Journal of the American Society for Psychical Research, 37, 57-83.

Taylor, J. (1980). Science and the supernatural - An investigation of such paranormal phenomena as psychic healing, clairvoyance, telepathy and precognition by a distinguished physicist and mathematician (1st ed.). New York: E.P. Dutton.

Taylor, S. E., \& Brown, J. D. (1988). Illusion and well-being: A social psychological perspective on mental health. Psychological Bulletin, 103(2), 193-210. https://doi.org/10.1037/0033-2909.103.2.193

Tetlock, P. E. (2017). Expert political judgment: How good is it? How can we know? Princeton, NJ: Princeton University Press.

Thalbourne, M. A. (1996). An attempt to predict precognition scores using transliminality relevant variables. Journal of the Society for Psychical Research, 62, 129-140.

Thalbourne, M. A. (1998). Transliminality: Further correlates and a short measure. Journal of the American Society for Psychical Research, 92(4), 402-419.

Thalbourne, M. A. (2010). The Australian Sheep-goat scale: Development and empirical findings. Australian Journal of Parapsychology, 10(1), 5-39. 
Thalbourne, M. A., Beloff, I., \& Delanoy, D. (1982). A test for the 'extraverted sheep versus introverted goats' hypothesis. In W. G. Roll, R. L. Morris, \& R. A. White (Eds.), Research in Parapsychology 1981 (pp. 155-156). Metuchen, NJ: Scarecrow Press.

Thalbourne, M. A., \& Delin, P. (1994). A common thread underlying belief in the paranormal, creative personality, mystical experience and psychopathology. Journal of Parapsychology, 58(1), 3-38.

Thalbourne, M. A., \& Delin, P. S. (1993). A new instrument for measuring sheep-goat variable: Its psychometric properties and factor structure. Journal of the Society for Psychical Research, 59(832), 172-186.

Thalbourne, M. A., \& Houran, J. (2003). Transliminality as an index of the sheep-goat variable. European Journal of Parapsychology, 18, 3-14.

Thalbourne, M. A., \& Maltby, J. (2008). Transliminality, thin boundaries, Unusual Experiences, and temporal lobe lability. Personality and Individual Differences, 44(7), 1617-1623. https://doi.org/10.1016/j.paid.2008.01.022

Thalbourne, M. A., \& Storm, L. (2012). Has the sheep-goat variable had its day?: Testing transliminality as a psi predictor. Australian Journal of Parapsychology, 12(1), 69-80.

Thalbourne, M. A., \& Storm, L. (2014). A further study of psychopraxia using the 'I Ching'. Australian Journal of Parapsychology, 14(2), 115-142.

Thorndike, E. L. (1898). Animal intelligence: An experimental study of the associative processes in animals. Psychological Review, 5(5), 551-553. https://doi.org/10.1037/h0067373

Tobacyk, J. J. (2004). A revised paranormal belief scale. International Journal of Transpersonal Studies, 23(1), 94-98. https://doi.org/10.24972/ijts.2004.23.1.94

Tobacyk, J. J., Nagot, E., \& Miller, M. (1988). Paranormal Beliefs and Locus of Control: A Multidimensional Examination. Journal of Personality Assessment, 52(2), 241-246. https://doi.org/10.1207/s15327752jpa5202_5

Tobacyk, J. J., \& Pirttilä-Backman, A.-M. (1992). Paranormal beliefs and their implications in university students from Finland and the United States. Journal of Cross-Cultural Psychology, 23(1), 59-71. https://doi.org/10.1177/0022022192231004 
Tobacyk, J. J., \& Wilkinson, L. V. (1991). Paranormal beliefs and preference for games of chance. Psychological Reports, 68(3 Suppl), 1088-1090. https://doi.org/10.2466/pr0.1991.68.3c.1088

Torrance, E. P. (1963). Preliminary manual for personal-social motivation inventory. Minneapolis, MN: University of Minnesota.

Ullman, M., Krippner, S., \& Vaughan, A. (1989). Dream telepathy: Experiments in nocturnal ESP. Jefferson, NC: McFarland \& Co.

Utts, J. (1987). Psi, statistics, and society. Behavioral and Brain Sciences, 10(4), 615616. https://doi.org/10.1017/S0140525X00054923

Utts, J. (1991). Replication and meta-analysis in parapsychology. Statistical Science, 6(4), 363-378. https://doi.org/10.1214/ss/1177011577

Utts, J. (1995). Response to Ray Hyman's report. Retrieved from http://www.ics.uci.edu/ jutts/response.html

Valentine, J. C., Pigott, T. D., \& Rothstein, H. R. (2010). How many studies do you need? A primer on statistical power for meta-analysis. Journal of Educational and Behavioral Statistics, 35(2), 215-247. https://doi.org/10.3102/1076998609346961

Vaughan, A., \& Houck, J. (1993). A" success" test of precognition and attitude toward the future. Journal of the Society for Psychical Research, 59, 259-268.

Vernon, D. J. (2015). Exploring precognition using a repetition priming paradigm. Journal of the Society for Psychical Research, 79(919), 65-79.

von Lucadou, W., Romer, H., \& Walach, H. (2007). Synchronistic phenomena as entanglement correlations in generalized quantum theory. Journal of Consciousness Studies, 14(4), 50-74.

Wagenmakers, E.-J., Marsman, M., Jamil, T., Ly, A., Verhagen, J., Love, J., ... Morey, R. D. (2018). Bayesian inference for psychology. Part I: Theoretical advantages and practical ramifications. Psychonomic Bulletin \& Review, 25(1), 35-57. https://doi.org/10.3758/s13423-017-1343-3

Wagenmakers, E.-J., Wetzels, R., Borsboom, D., \& van der Maas, H. L. J. (2011). Why psychologists must change the way they analyze their data: The case of psi: Comment on Bem (2011). Journal of Personality and Social Psychology, 100(3), 426-432. https://doi.org/10.1037/a0022790

Wagenmakers, E.-J., Wetzels, R., Borsboom, D., van der Maas, H. L. J., \& Kievit, R. A. (2012). An agenda for purely confirmatory research. Perspectives on 
Psychological Science, 7(6), 632-638.

https://doi.org/10.1177/1745691612463078

Wallis, R. (Ed.). (1979). On the margins of science: The social construction of rejected knowledge. Keele: University of Keele.

Wang, T., van Loon, P. R., van den Assem, M., \& van Dolder, D. (2016). Number preferences in lotteries. Judgment and Decision Making, 11(3), 243-259. https://doi.org/10.2139/ssrn.2657776

Watt, C. (2005). Parapsychology's contribution to psychology: A view from the front line. Journal of Parapsychology, 69(2), 215-231.

Watt, C. (2016a). A prospective meta-analysis of pre-registered ganzfeld ESP studies. Retrieved from https://koestlerunit.wordpress.com/study-registry/registeredstudies/

Watt, C. (2016b). Parapsychology. London, England: Oneworld Publications.

Watt, C., \& Kennedy, J. E. (2015). Lessons from the first two years of operating a study registry. Frontiers in Psychology, 6, 173. https://doi.org/10.3389/fpsyg.2015.00173

Watt, C., \& Kennedy, J. E. (2017). Options for prospective meta-analysis and introduction of registration-based prospective meta-analysis. Frontiers in Psychology, 7, 2030. https://doi.org/10.3389/fpsyg.2016.02030

Watt, C., \& Nagtegaal, M. (2000). Luck in action? Belief in good luck, psi-mediated instrumental response, and games of chance. Journal of Parapsychology, 64(1), $33-52$.

Watt, C., \& Ramakers, P. (2003). Experimenter effects with a remote facilitation of attention focusing task: a study with multiple believer and disbeliever experimenters. Journal of Parapsychology, 67(1), 99-116.

Watt, C., \& Wiseman, R. (Eds.). (2005). Parapsychology. Burlington, VT: Routledge. Wilson, D. B., \& Shadish, W. R. (2006). On blowing trumpets to the tulips: To prove or not to prove the null hypothesis - comment on Bösch, Steinkamp, and Boller (2006). Psychological Bulletin, 132(4), 524-528. https://doi.org/10.1037/00332909.132.4.524

Wilson, K., \& French, C. (2006). The relationship between susceptibility to false memories, dissociativity, and paranormal belief and experience. Personality and Individual Differences, 41(8), 1493-1502. https://doi.org/10.1016/j.paid.2006.06.008 
Wilson, K., \& French, C. (2014). Magic and memory: Using conjuring to explore the effects of suggestion, social influence and paranormal belief on eyewitness testimony for an ostensibly paranormal event. Frontiers in Psychology, 5, 1289. https://doi.org/10.3389/fpsyg.2014.01289

Wilson, S., \& Hamlin, I. (2007). Implicit learning in a card prediction task. European Journal of Parapsychology, 22(1), 3-29.

Winkelman, M. (1981). The effect of formal education on extrasensory abilities: The Ozolco study. Journal of Parapsychology, 45(4), 321-336.

Wiseman, R. (2010). 'Heads I win, tails you lose': How parapsychologists nullify null results. Retrieved from https://www.csicop.org/si/show/heads_i_win_tails_you_loser_how_parapsycho logists_nullify_null_results

Wiseman, R., \& Greening, E. (2002). The mind machine: A mass participation experiment into the possible existence of extra-sensory perception. British Journal of Psychology, 93, 487-499. https://doi.org/10.1348/000712602761381367

Wiseman, R., \& Schlitz, M. (1997). Experimenter effects and the remote detection of staring. Journal of Parapsychology, 61(3), 205-207.

Wiseman, R., \& Smith, M. D. (2002). Assessing the role of cognitive and motivational biases in belief in the paranormal. Journal of the Society for Psychical Research, 66, 157-166.

Yarkoni, T. (2011). The psychology of parapsychology, or why good researchers publishing good articles in good journals can still get it totally wrong. Retrieved from http://www.talyarkoni.org/blog/2011/01/10/the-psychology-ofparapsychology-or-why-good-researchers-publishing-good-articles-in-goodjournals-can-still-get-it-totally-wrong/

Zdrenka, M. (2017). Individual difference correlates of lottery success. Retrieved from http://www.koestlerparapsychology.psy.ed.ac.uk/Documents/KPU_Registry_1038.pdf

Zdrenka, M., \& Wilson, M. S. (2017). Individual difference correlates of psi performance in forced-choice precognition experiments (1945-2016). Journal of Parapsychology, 81(1), 9-32. 
Zilberman, M. S. (1995). Public numerical lotteries: An international parapsychological experiment covering a decade. Journal of the Society for Psychical Research, 60, 149-160.

Zusne, L., \& Jones, W. H. (1989). Anomalistic psychology: A study of magical thinking (2nd ed.). Hillsdale, NJ: Psychology Press. 


\section{META-ANALYSIS (STUDY I) PAPERS NOT CITED}

Hitchman, G. A. M., Pfeuffer, C. U., Roe, C. A., \& Sherwood, S. J. (2016). The effects of experimenter-participant interaction qualities in a goal-oriented nonintentional precognition task. Journal of Parapsychology, 80(1), 45-69.

Hitchman, G. A. M., Roe, C. A., \& Sherwood, S. J. (2015). The relationship between lability and performance at intentional and nonintentional versions of an implicit PMIR-type psi task. Journal of Parapsychology, 79(1), 65-86.

Hitchman, G. A. M., Sherwood, S. J., \& Roe, C. A. (2015). The relationship between latent inhibition and performance at a non-intentional precognition task. Explore: The Journal of Science and Healing, 11(2), 118-126. https://doi.org/10.1016/j.explore.2014.12.004

Luke, D., Zychowicz, K., Richterova, O., Tjurina, I., \& Polonnikova, J. (2012). A sideways look at the neurobiology of psi: Precognition and circadian rhythms. NeuroQuantology, 10(3), 580-590. https://doi.org/10.14704/nq.2012.10.3.614

Nielsen, W. (1970). Relationships between precognition scoring level and mood. Journal of Parapsychology, 34(2), 93-116.

Palmer, J. (2009). Decision augmentation in a computer guessing task. Journal of Parapsychology, 73, 119-135.

Ryzl, M. (1968a). Precognition scoring and attitude. Journal of Parapsychology, 32(3), 183-189.

Ryzl, M. (1968b). Precognition scoring and attitude toward ESP. Journal of Parapsychology, 32(1), 1-8. 


\section{APPENDIX A}

Table A1

Number of Studies for Each Individual Difference Measure that Reported NonSignificance Without Providing Any Other Statistical Information

\begin{tabular}{lc}
\hline Individual Difference Measure & $\begin{array}{c}\text { Number of Non-Significant Studies Estimated } \\
\text { as } r=.00\end{array}$ \\
\hline Belief in ESP & 5 \\
Extraversion & 3 \\
Neuroticism & 2 \\
Agreeableness & 2 \\
Spontaneous Experiences & 2 \\
Openness to Experience & 1 \\
Dream Recall & 1 \\
Conscientiousness & 1 \\
Temporal Lobe Dysfunction & 1 \\
Cerebral Lateralization & 1 \\
\hline
\end{tabular}

Table A2

68\% and $80 \%$ Confidence Intervals for All Individual Difference Measures MetaAnalysed

\begin{tabular}{lcc}
\hline Individual Difference Measure & $\begin{array}{c}6 \% \text { Confidence } \\
\text { Intervals }\end{array}$ & $\begin{array}{c}80 \% \text { Confidence } \\
\text { Intervals }\end{array}$ \\
\hline Chance Belief & $.03, .25$ & $-.01, .28$ \\
Belief in ESP & $.10, .17$ & $.09, .18$ \\
Luck Belief & $.07, .20$ & $.05, .21$ \\
Perceptual Defensiveness & $.06, .19$ & $.04, .20$ \\
Providence Belief & $.05, .20$ & $.02, .22$ \\
Openness to Experience & $.07, .16$ & $.06, .17$ \\
Extraversion & $.05, .11$ & $.04, .12$ \\
Dream Recall & $.01, .13$ & $-.004, .14$ \\
Intelligence & $-.19, .07$ & $-.23, .10$ \\
Emotional Reactivity & $-.11, .23$ & $-.16, .28$ \\
Creativity & $.01, .12$ & $-.04, .13$ \\
Perceived Luckiness & $-.03, .15$ & $-.05, .18$ \\
Conscientiousness & $-.02, .13$ & $-.04, .15$ \\
Neuroticism & $-.01, .12$ & $-.03, .14$ \\
Religiosity & $-.15, .05$ & $-.18, .07$ \\
Future-Oriented & $-.04, .15$ & $-.07, .17$ \\
Fortune Belief & $-.03, .10$ & $-.05, .12$ \\
Agreeableness & $-.04, .08$ & $-.05, .10$ \\
Pro Attitude & $-.07, .03$ & $-.08, .05$ \\
Time Belief as Dynamic & $-.03,-.01$ & $-.03,-.01$ \\
Transliminality & $-.06, .08$ & $-.08, .10$ \\
Spontaneous Experiences & $-.04, .03$ & $-.05, .04$ \\
Temporal Lobe Dysfunction & $-.10, .09$ & $-.13, .12$ \\
\hline
\end{tabular}




\section{APPENDIX B}

- Ethics Application (Study II)

- Information Sheet for Participants (Study II)

- Debrief for Participants (Study II)

- Survey Questions (Study II) 


\section{RM ResearchMaster \\ (E)}

Human Ethics Application

Application ID :

Application Title :

Date of Submission :

Primary Investigator :

Other Investigators :

Deo0022146 Pem's (2011) Precognitive Detection of Erotic Stimul

N/A

Marco Zdrenka

A/Prof Marc Wilson 


\section{Research Form}

Application Type

What type of application is this?

Research Only

\section{Information}

\section{Welcome to the Human Ethics Application Form}

The following advice will assist you in completing this process:

\section{Help contact}

For information about Human Ethics, go to the Human Ethics web page.

For help, please email the Ethics Administrator.

\section{Policy}

You must read the Human Ethics Policy before beginning your application. Appendix B of the Policy contains a sample consent form, information sheet, and transcribing confidentiality form which may be useful (see last page).

Health research may require HDEC approval. To find out if your research requires this, read the HDEC Guidelines or contact the chairperson of your committee for clarification. If your research does require HDEC approval, a copy of the application must be sent to the Administrator of the HEC. Evidence of approval should also be sent to the HEC

Student research

If you are a student, check with your supervisor before filling in this form. You may need to complete School requirements before applying for ethical approval. Student applications will be automatically forwarded to supervisor(s) and then Head of School/Delegate for approval when the form is submitted. Once the Head of School has

\section{Technical}

This online system works best on Internet Explorer and Safari. It may not work on your iPad or tablet.

A guide to using this online form, which includes a workflow showing how the approval process works, can be downloaded here.

If your application involves other researchers, you can use the Comments function of this form to communicate about the application with each other. Click on the Application Comments or Page Comments icon on the top right of the screen to view and add comments. Comments left on the
School and committee reviewers, so remember to delete any private comments before submitting the form.

\section{Process}

All applications will be automatically forwarded to the Head of School for review when the application is submitted. Once the Head of School has approved it, the form will be automatically forwarded for committee review.

You will normally receive an outcome of the review of your application within three weeks, unless you apply during an advertised close-down period (for instance, applications may not be reviewed in December and January). NO part of the research requiring ethical approval may commence prior to approval being given

To apply for an amendment or extension to an approved application, open the approved form and click on Apply for amendment/extension. You will then be able to complete the Amendment/Extension page and resubmit the form.

Application Details

1. Ethics category code*

Human

Application ID

0000022146 
2. Please select the appropriate committee below. Please note that:

- Education applications are now handled by the Human Ethics Committee.

School of Psychology Ethic

3. The following question is meant to help applicants consider their research application and any protocols that should be uploaded and to help to help committee members review the application.

Indicate all answers that apply. Please indicate whether the research planned for this application:

$\square$ Includes equipment or financial resources that have not yet been sourced

$\square$ Is similar to prior research of yours (with VUW ethical approval)

Recruits participants that may not be mentally capable of understanding information for informed con

$\square$ Uses audio or video recording of participants

$\square$ Uses noninvasive physiological procedures (e.g., EEG, heart rate monito

$\square$ Uses equipment (e.g., TMS) that may temporarily alter mental function

Uses sensitive materials or procedures (e.g., sexually explicit or disturbing imagery, depictions of

$\square$ Collects human fluid samples (e.g., saliva)

$\square$ Administers substances (e.g., food, alcohol, placebo pill) to be ingested by participants

$\square$ Uses equipment or procedures that are likely to produce some physical discomfort for participants

$\square$ Uses materials or procedures that are likely to put participants in some mental distress

Recruits participants from the IPRP, summer pool or psychology research methods courses

$\square$ Recruits participants from within a psychology course in which you will be involved

$\square$ Recruits participants via external organisations

$\square$ Recruits participants from internet pools (e.g., Mechanical Turk)

$\square$ Recruits participants by written advertisements/invitations, including via Facebook

$\square$ Recruits participants by personally soliciting in a public space

Recruits participants by some means not listed here

Recruits participants under the age of 18

4. Title of project*

Replication of Bem's (2011) Precognitive Detection of Erotic Stimul

School or research centre*

Psychology

5. Please list all personnel involved in this project. Ensure that all are listed with the correct role. If you are a student, do not add your supervisor here: you will be asked to add this information on the next page.

Please ensure that only one person is listed as Principal Investigator.

To add a person, search for their Victoria ID if known, otherwise either their first or last name (whichever is the most unusual). Click on the magnifying glass to search for results. Press the green tick at the bottom right corner to save the person record.

Add anybody who is involved in this project as:

- Associate Investigato

- Other Research

- PhD Student

- Masters Student

Click on the help button if you are having difficulty adding people to the list.

\begin{tabular}{|l|l|l|}
\hline 1 & Given Name & Marco \\
\hline & Surname & Zdrenka \\
\hline & Full Name & Marco Zdrenka \\
\hline & AOU & PSYC \\
\hline & Position & Principal Investigator \\
\hline & Primary? & Yes \\
\hline
\end{tabular}

6. Are any of the researchers from outside Victoria?*

$\mathrm{O}$ Yes

No

7. Is the principal investigator a student?*

$\odot$ Yes

No 
Next time you save this form or move to a new page, a Student Research page will appear after this one. Please complete the two questions on the Student Research page.

Student Research

7a. What is your course code (e.g. ANTH 690)?*

PSYC 690

7b. Please add your primary supervisor (the supervisor who should review this application).

If your supervisor is also the Head of School or the school ethics officer, you will need to discuss with your School who should approve this application as Head of School or delegate. The supervisor and Head of School or delegate must not be the same person.

To add your supervisor, search for their Victoria ID if known, otherwise either their first or last name (whichever is the most unusual).

Press the green tick at the bottom right corner to save the person record:

\begin{tabular}{|l|l|l|}
\hline 1 & Given Name & Marc \\
\hline & Surname & Wilson \\
\hline & Full Name & A/Prof Marc Wilson \\
\hline & AOU & PSYC \\
\hline & Position & Supervisor \\
\hline
\end{tabular}

If your supervisor is also the Head of School, you will need to assign a different person to the Head of School or Delegate role on the Signoff page.

7c. What is your email address? (this is needed in case the committee needs to contact you about this application)*

Marco.Zdrenka@vuw.ac.nz

Note that system-generated emails (eg approval notifications) will not necessarily come to this address. System-generated emails will come to the email address stored for you in Note that system-generated emails (eg approval noufications) will not necessarily come to this address. System-generated emails will come to the email address stored for you

Project Details

9. Describe the objectives of the project*

The aims of this proct are

a) replicate Bem's (2011) precognitive detection of erotic stimuli experiment which found that participants were able to anticipate, at a greater

b) to investigate whether individual differences in personality or cognition are able to predict performance in this task.

10. Describe the benefits and scholarly value of the project*

Bem's (2011) findings have led to a lot of controversy in Psychology, with claims that he may have arbitrarily stopped his experiments when the results were in his favour, that his statistical methods were unsuitable and incorrectly used, and that his experiments suffer from the file-drawer effect and are not actually replicable.

This project attempts to address these criticisms by pre-specifying the participants, measures, and analyses beforehand. Its outcome will add to a

precognitive effect or whether the results were merely artifactual.

11. Describe the method of data collection. Note that later in this form, in the Documents section, you will need to upload any relevant documentation such as interview schedule, survey, questionnaires, focus group rules, observation protocols etc. Delays are likely if the interview questions are missing from the Documents section. *

Firstly, participants will be seated in front of a computer and given an explanation about the experiment

Participants will then complete brief measures of personality (Mini-IPIP6 measure of personality; Sibley, Luyten, Purnomo, Mobberley, Wootton, Hammond et al., 2011), paranormal belief (Paranormal Belief Scale (PBS); Tobacyk, 1997), sensation seeking (Stimulus Seeking Scale (SSS); Bem, performance in precogition. The SPQ and PBS have been used extensie, in IPR.

Following this, a 3-minute relaxation period will then begin on the computer, followed by 36 trials. In each trial, participants will guess whether the left or right side of the screen contains a picture. If a picture is correctly identified, it will be revealed.

Most of the pictures selected for this experiment will be used from the International Affective Picture System (IAPS; Lang \& Greenwald, 1993) or those that were previously used by Bem (2011).

Overall, participation will last approximately 30 minutes.

12. Does your research have more than one phase that requires HEC approval?

$\mathrm{O}$ Yes

- No

Key Dates

If approved, this application will cover this research project from the date of approval

$7 / 21 / 2015$

Page 4 / 9 
13. Proposed end date for data collection*

$01 / 03 / 2018$

14. Proposed end date for research project as a whole* $01 / 03 / 2020$

Proposed source of funding and other ethical considerations

15. Indicate any sources of funding, including self-funding (self-funding means that you are paying for research costs such as travel, postage etc. from your own funds) (tick all that

$\square$ Internally funded

$\square$ Externally funded

$\square$ Self-funded

16. Is any professional code of ethics to be followed?*

○ Yes

No

16a. Name the professional code(s) of ethics *

American Psychological Association

17. Is ethical approval required from any other body?*

$O$ Yes

18 Depending on the characteristics of your participants or location of the research, you may need to arrange permission from another body or group before proceeding. If this is the case, explain and describe how you are addressing this*

N/A

\section{Treaty of Waitangi}

19. How does your research conform to the University's Treaty of Waitangi Statute? (you can access the statute from Victoria's Treaty of Waitangi page)*

The focus of the study is not specifically on Maori participants, but participation from all eligible volunteers is welcomed. With that in mind, guideline $4.3[\mathrm{x}]$ is most relevant here ("Ensures that the University does not discriminate unfairly against any person").

Information about participants

20. How many participants will be involved in your research? If you are using records (e.g. historical), please estimate the number of records 200

21. What are the characteristics of the people you will be recruiting?*

First-year psychology students. Participation will be restricted to those who are at least 18 years of age.

22. Are you specifically recruiting any of the following groups?

- Māori

- Children/youth

- People who are offenders and/or victims of crime

People with disabilities

- People in residential care

Please indicate below.*

$\odot$ Yes

No

22a. Which of these groups will you be recruiting? (tick all that apply)* 
$\square$ People who are offenders and/or victims of crime

$\square$ People with disabilities

People in residential care

$\square$ People who are refugees

23. Have you undertaken any consultation with the groups from which you will be recruiting?* No

24. Provide details of consultation you have undertaken or are planning* N/A

25. Outline the method(s) of recruitment you will use for participants in your study* Participants will be invited to participate as part of 100 -level IPRP, following IPRP protocols.

26. Will your participants receive any gifts/koha in return for participating?*

$\mathrm{O}$ Yes

27. Will your participants receive any other assistance (for instance, meals, transport, release time or reimbursements)?* $\mathrm{O}$ Yes

No

28. Will your participants experience any special hazard/risk including deception and/or inconvenience as a result of the research?* ○ Yes

No

28a. Give details and indicate how you will manage this*

As the study includes presentation of erotic images, participation will be restricted to those students who indicate in IPRP pre-test that they are happy to view explicit images.

Participants will not be told exactly what is being tested beforehand, which constitutes mild deception. This is unavoidable in testing the research hypotheses. However, all participants will be thoroughly debriefed, and can contact Marco Zdrenka or A/Prof Marc Wilson with any questions.

29. Is any other party likely to experience any special hazard/risk including breach of privacy or release of commercially sensitive information?* $\mathrm{O}$ Yes

No

30. Do you have any professional, personal, or financial relationship with prospective research participants?* ○ Yes

O No

30a. Give details and indicate how you will manage this*

A/Prof Marc Wilson is the Course Coordinator for IPRP, so a professional relationship exists with prospective research participants from the IPRP pool. However, as participants will be interacting with Marco Zdrenka rather than A/Prof Marc Wilson, this does not pose much of a problem.

31. What opportunity will participants have to review the information they provide? (tick all that apply)*

$\square$ They will be given a transcript of their interview

$\square$ They will be given a summary of their interview

$\square$ other

They will not have an opportunity to review the information they provide

31b. Please give details about why the participants will not be able to review the information they provide*

As the experiment relies on the initial responses of participants rather than revised responses, a review would be inappropriate. Also, student IDs are soon removed from the responses, so it would be difficult to locate the data from any one participant.

Informed consent

32. Will participation be anonymous? 'Anonymous' means that the identity of the research participant is not known to anyone involved in the research, including (It not possible for the researchers to identify whether the person took part in the research, or to subsequently identify people who took part (e.g., by recognising them in different settings by their appearance, or by wh 
O Yes

○ No

33. Will contributions of participants be confidential? Confidential means that those involved in the research are able to identify the participants but will not reveal their identity to anyone outside the research team. Researchers will also take reasonable precautions to ensure that participantsi identities cannot be linked to their responses in the future.

Y) Yes

No

33a. How will confidentiality be maintained in terms of access to the research data? (tick all that apply)*

$\square$ Access to the research will be restricted to the investigator

Access to the research will be restricted to the investigator and their supervisor (student research

$\square$ Focus groups will have confidentiality ground rules

$\square$ Transcribers will sign confidentiality forms

$\square$ Other

33c. Please provide further details about how confidentiality will be maintained in terms of reporting of the data*

Data are effectively anonymous once student ID information is removed (student ID information will be sought initially, to credit participants for IPRP).

33b. How will confidentiality be maintained in terms of reporting of the data? (tick all that apply)*

$\square$ Pseudonyms will be used

$\square$ Participants will be named only in a list of interviewees

Data will be aggregated and so not reported at an individual level

Participants will be referred to by role or association with an organisation rather than by name

Names will be confidential, but other identifying characteristics may be published with consent

$\square$ other

33c. Please provide further details about how confidentiality will be maintained in terms of reporting of the data*

See 33 c above.

34. How will informed consent be obtained? (tick all that apply to all phases of the research you are describing in this application)*

$\square$ Informed consent will be implied through voluntary participation (anonymous research only)

$\square$ Informed consent will be obtained through a signed consent form

$\square$ Informed consent will be obtained by some other method

\section{Access, storage, use, and disposal of data}

35. What procedures will be in place for the storage of, access to and disposal of data, both during and at the conclusion of the research? (tick all that apply)*

$\square$ All written material will be kept in a locked file; access restricted to investigator(s)

All electronic information will be password-protected; access restricted to the investigator(s)

$\square$ All questionnaires, interview notes and similar materials will be destroyed

$\square$ Any audio or video recording will be returned to participants and/or electronically wiped

$\square$ other procedures

35a. Describe the procedures for the storage of and access to the data*

Open access; publicly available following the removal of identifying information.

35b. Will the data be destroyed immediately after the conclusion of the research?*

$\mathrm{O}$ Yes

○ No

35c. How many years after the conclusion of the research will the materials be destroyed? 999.00

36. If data and material are not to be destroyed, indicate why and the procedures envisaged for ongoing storage and security In order to be transparent and avoid the criticisms of Bem's (2011) findings, the data must be made available and accessible to anyone who
wants it. However, it will not affect the participants as there will be no identifying information and they will have provided consent for this use.

\section{Dissemination}

37. How will you provide feedback to participants?*

Students will receive a debriefing statement as well as being verbally debriefed. 
38. How will results be reported and published? Indicate which of the following are appropriate. The proposed form of publications should be indicated on the information sheet and/or consent form*

7 Publication in academic or professional journals

$\square$ Dissemination at academic or professional conferences

Availability of the research paper or thesis in the University Library and Institutional Repositon

$\square$ Other

39. Is it likely that this research will generate commercialisable intellectual property? (check the help text for more information about IP)

$\mathrm{O}$ Yes

○ No

Documents

40. Please upload any documents relating to this application. Ensure that your files are small enough to upload easily, and in formats which reviewers can easily download and review. To replace a document, click the tick in the column to the right of the document title. A green arrow will appear - click this arrow to upload a new document. To add a new ecument click on 'Add New Document', at top right of the documents window. Then
green arrow will appear to the right of the file name which allows you to upload the new file."

This question is not answered.

\section{Getting feedback on your application}

You can seek feedback on your draft application, for instance from a mentor or a school Ethics representative before submitting it for review.

There are two ways of doing this:

1. Emailing your application to someone

You can email your application and any associated documents to another person at Victoria. To do this:

Click on the Action tab (on the left of the screen)

. Click on Email application

Search for the person using either their first name or their last name (whichever is the most unusual)

Select the documents to include from the Document list (eg the Application PDF)

If you wish to send your application to someone outside Victoria, one option is emailing the application to yourself and then forwarding it

\section{Assigning a peer reviewer}

You can add someone to the form as 'peer reviewer'. This means that they will be able to access your form by logging onto ResearchMaster. They will also be able to comment on your form online. If you are a student, don't add your supervisor to the form as a peer reviewer - to get supervisor feedback, submit the form. Your superviso

Click on the Review tab on the left of the screen

Search for the person using their person code if known, or either their first name or their last name (whichever is the most unusual)

. You may then also want to send the peer reviewer a notification, by clicking on Notify Peer Reviewer on the Actions tab

\section{Checklist}


Please check the information below and tick the box at the bottom of the page. Then follow the instructions to submit.

- Have you read the Human Ethics Policy?

- Have you incuded an information sheet for participants which explains:

the nature and purpose of your research;

the proposed use of the material collected

who will have access to the material collected

whether the data will be kept confidential to you

Does your information sheet also include:
a statement about participants' right to withdraw and the final date for doing so (and is this also referred to in the consent form)?

a statement confirming that the research has been approved by Victoria University of Wellington Human Ethics Committe

a statement about the destruction of the data at the end of the project?

- Contact details for the HEC Convener should participants have ethics queries? (AProf Susan Corbett, email susan.corbett@vuw.ac.nz, telephone +64-4-463 5480)

- Have you used your VuW email address?

- Have you included a written consent form?

If not, have you explained on the application form why you do not need to get written consent?

- Are your information sheets and consent forms on VUW letterhead?

- Have you included a copy of any questionnaire or interview schedule you propose using?

I have gone through the checklist and completed all the relevant tasks. *

$\square$ Yes

This question is not answered

Signoff

This section records sign-off by all other researchers involved in the project (the other team members listed at Q.6). Principal investigators do not need to complete this section - you signoff by submitting the application.

If co-researchers are external to Victoria University they may be unable to access this site. In this instance, the Principal Investigator may sign off on their behalf. Please upload evidence of the co-researchers' signoff (e.g., a scanned email) to the Documents page.

To sign off, do ALL 5 of the following 5 steps::

Click on the pencil icon on the far right of the line with your name on it

Click on I Accep

Click on the green tick icon on the bottom of the signoff window

5. Go to the Actions tab and click on 'Notify lead researcher that signoff is complete'

This question is not answered.

42. Please add the Head of School (or delegate. e.g. school ethics officer) who should approve this application. This will be your own Head of School, or the person in your School responsible for approving Ethics applications. The form will be forwarded to this person automatically once it is submitted. Please check with your School administration team if you are unclear who should be assigned this role. Adding the wrong person could lead to delays in processing your application.

If you are a student, the Head of School or delegate must not be the same person as your supervisor.

Once you've searched for your Head of School/delegate, click on the green tick to add them, and then also save the application before submitting.

Heads of School or delegates should process this form by clicking on the Actions tab and either approve it, or return it to the researcher for further changes. *

This question is not answere.

Please ensure that you save your application before submitting it. Once you have saved your application, to submit it, click on 'Actions' on the left hand side of the screen and then 'Submit for review'.

If you are a student, your application will go to your supervisor and then Head of School for approval once you submit it. If you are a staff member, your

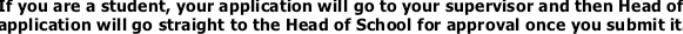

If you have any feedback about this online form, please email it to ethicsadmin@vuw.ac.nz 


\section{Ethics Application attachment}

The following experimental protocols are being pre-specified before the experiment begins, as per Wagenmakers, Wetzels, Borsboom, and van der Maas' (2012) recommendation to researchers who wish to avoid the common criticisms of Bem's (2011) experiments:

1) The number of participants in this experiment will be 200.

2) No data will be transformed during the analyses.

3) The analyses that will be performed are one-sample $t$ tests across sessions comparing the hit rate of erotic images with non-erotic images and a nonparametric binomial test on the overall proportion of hits across all trials and sessions (tested against a null of .5). Individual difference correlations and $t$ tests will also be conducted.

4) Bayesian $t$ tests will also be performed on the data. 
Information Sheet

Marco Zdrenka

\title{
PhD Student
}

Marco.Zdrenka@vuw.ac.nz

\section{What is the purpose of this research?}

- This experiment tests for Extra Sensory Perception (ESP).

\section{Who is conducting the research?}

- This research is being conducted by Marco Zdrenka, a PhD student at Victoria University of Wellington, and is supervised by A/Prof Marc Wilson of the School of Psychology (Marc.Wilson@vuw.ac.nz). This study has been approved by the School of Psychology Human Ethics Committee under the delegated authority of the Victoria University Human Ethics committee (\#0000022146).

\section{What is involved if you agree to participate?}

- If you agree to participate in this study, you will complete a survey of your views, experiences, and beliefs.

- You must be at least 18 years of age to participate in this study.

- You will also complete an experiment that takes about 20 minutes and is run completely by computer. First you will answer a couple of brief questions. Then, on each trial of the experiment, pictures of two curtains will appear on the screen side by side. One of them has a picture behind it; the other has a blank wall behind it. Your task is to click on the curtain that you feel has the picture behind it. The curtain will then open, permitting you to see if you selected the correct curtain. There will be 36 trials in all.

- Participation is entirely voluntary. If you sign this form, you will be consenting to participation.

- During the research you are free to withdraw at any point before completion.

\section{Erotic Imagary}

- Several of the pictures contain explicit erotic images (e.g. couples engaged in nonviolent but explicit consensual sexual acts). If you object to seeing such images, you should not participate in this experiment.

\section{Privacy and Confidentiality}

- Your responses will be kept completely confidential and the only identifying information we require is your Student ID (to give you credit for IPRP). Your identifying information will be removed before any data is analysed.

- In order to be transparent, the data will be publically available for anyone to access. In accordance with the requirements of some scientific journals and organisations, your data may be shared with other competent researchers, and/or used in other related studies.

- You will not be identifiable in the results or any other presentation or publication of them.

- We will keep your responses (electronically) for up to 999 years.

- A copy of the coded data will remain in the custody of Marco Zdrenka and A/Prof Marc Wilson in his office/laboratory.

\section{What happens to the information that you provide?}

- The data you provide may be used in a manuscript submitted for publication in a scientific journal, or presented at a scientific conference.

\author{
THE PURPOSE OF THE DATA IS NOT FOR INAPPROPRIATE CHARACTERISATION OF \\ INDIVIDUALS OR GROUPS ON THE BASIS OF THEIR BELIEFS.
}

Student ID number:

Signature: 


\section{Study Debrief}

Thank you for participating in this experiment.

As you can tell from the procedure itself, we are interested in whether people can use ESP to detect whether the picture will be on the left or on the right. What is not obvious from the procedure, however, is that this experiment is specifically designed to test for precognition, a form of ESP in which a person can anticipate the future.

To do this, the computer doesn't actually decide the placement of the picture until after you have already made your choice. Before you make your choice, the computer itself doesn't yet know where the picture will be. Instead, it waits until you have already made your choice and then it flips a virtual coin to decide whether to put it on the left or the right.

If there is no ESP, then we would expect people to see the picture on approximately $50 \%$ of the trials by pure chance. So, if people are successful at seeing the picture on significantly more than $50 \%$ of the trials, it would provide support for precognition.

Some of the trials included erotic stimuli, while others included non-erotic positive and negative stimuli. The use of erotic pictures in this type of research is traditional, as reinforcement for correct "precognitive" guesses.

Thank you again for participating in this research. If you have any queries, comments, or criticisms of this research, please contact us on one of the following email addresses:

Marco Zdrenka (Marco.Zdrenka@vuw.ac.nz) or A/Prof Marc Wilson (Marc.Wilson@vuw.ac.nz).

If there was an aspect of this research that bothered you and was not addressed here, please feel free to contact us. It may also help to talk to a friend or relative, or make an appointment with one of our Student Services (e.g., Student Health, Student Counselling) via their website, http://www.victoria.ac.nz/students/support.

If you have questions about the ethical approval of this study, please contact Associate Professor Susan Corbett (susan.corbett@vuw.ac.nz, telephone +6444635480 ). 
TE WHARE WĀNANGA O TE ŪPOKO O TE IKA A MĀUI

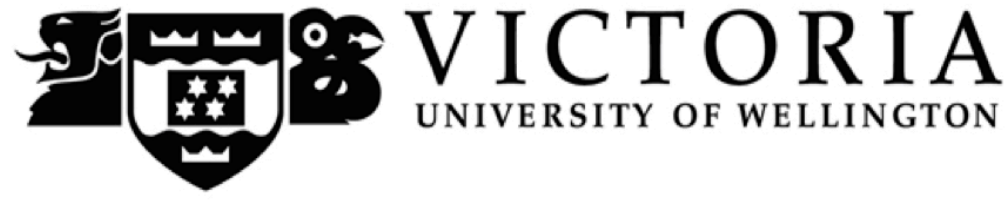

Vivid Experiences

$1 / 1$

The aim of this set of questions is to get a sense of how common various 'vivid' experiences are in the population of psychology students. These questions have ben asked of thousands of people around the world, and we'd like to know about your personal experience.

So we can credit you for your participation, and match your responses here to your participation in the other task(s) in this session, please tell us your Student ID number.

WE WILL DELETE THIS INFORMATION AFTER CREDITING AND DATA-MATCHING.

The aim of this set of questions is to get a sense of how common various 'vivid' experiences are in the population of psychology students. Because this is about your experiences, there's no such thing as a right or wrong answer - the best answer is whatever makes sense to you.

Read through each statement and tell us if you've had this experience ('Yes') or not ('No')

I have occasionally had the feeling that a TV or radio broadcaster
knew I was listening to him.
I have felt that there were messages for me in the way things were
arranged, like in a store window.

Things sometimes seem to be in different places when I get home, 
even though no one has been there.

I have never doubted that my dreams are the products of my own mind.

I have noticed sounds on my records that are not there at other times.

I have had the momentary feeling that someone's place has been taken by a look alike.

I have never had the feeling that certain thoughts of mine really belonged to someone else.

I have wondered whether the spirits of the dead can influence the living.

At times I perform certain little rituals to ward off negative influences.

I have felt that I might cause something to happen just by thinking too much about it.

At times, I have felt that a professor's lecture was meant especially for me.

I have sometimes felt that strangers were reading my mind.

If reincarnation were true, it would explain some unusual experiences I have had.

I sometimes have a feeling of gaining or losing energy when certain people look at me or touch me.

It is not possible to harm others merely by thinking bad thoughts about them.

\section{Continued...}

I have sometimes sensed an evil presence around me, although I could not see it.

People often behave so strangely that one wonders if they are part of an experiment.

The government refuses to tell us the truth about flying saucers.

I almost never dream about things before they happen.

I have sometimes had the passing thought that strangers are in love with me. 
The hand motions that strangers make seem to influence me at times.

Good luck charms don't work.

I have sometimes been fearful of stepping on sidewalk cracks.

Numbers like 13 and 7 have no special powers.

I have had the momentary feeling that I might not be human.

I think I could learn to read others' minds if I wanted to.

Horoscopes are right too often for it to be a coincidence.

Some people can make me aware of them just by thinking about me.

I have worried that people on other planets may be influencing what happens on Earth.

When introduced to strangers, I rarely wonder whether I have known them before.

\section{Continued...}

At times I perform certain little rituals to ward off negative influences

I have experienced an altered state of consciousness in which I felt that I became cosmically enlightened

At the present time, I am very good at make-believe and imagining

I have felt that I had received special wisdom, to be communicated to the rest of humanity

I have sometimes sensed an evil presence around me, although I could not see it

My thoughts have sometimes come so quickly that I couldn't write them all down fast enough

It is sometimes possible for me to be completely immersed in nature or in art and to feel as if my whole state of consciousness has somehow temporarily been altered

Often I have a day when indoor lights seem so bright that they bother my eyes

I have experienced an altered state of consciousness which I believe utterly transformed (in a positive manner) the way I looked at 
myself

I think that I really know what some people mean when they talk about mystical experiences

I have gone through times when smells seemed stronger and more overwhelming than usual

I can clearly feel again in my imagination such things as: the feeling of a gentle breeze, warm sand under bare feet, the softness of fur, cool grass, the warmth of the sun and the smell of freshly cut grass

A person should try to understand their dreams and be guided by or take warnings from them

At times I somehow feel the presence of someone who is not physically there

For several days at a time I have had such a heightened awareness of sights and sounds that I cannot shut them out

I sometimes have a feeling of gaining or losing energy when certain people look at me or touch me

When listening to organ music or other powerful music, I sometimes feel as if I am being lifted up into the air

There follows a list of personality characteristics. Please look at each one, and indicate how accurately it describes you.

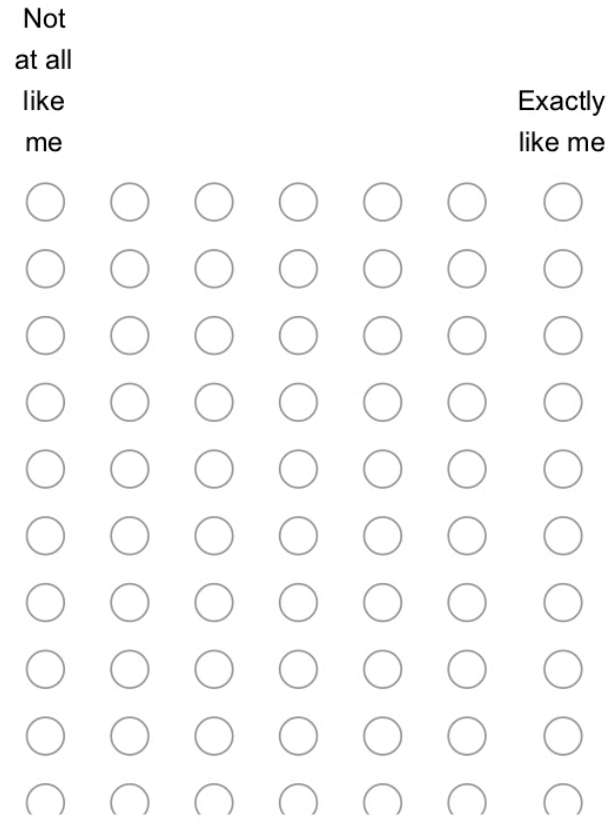


sympathetic, warm

Disorganized, careless

Calm, emotionally stable

Masculine

Conventional, uncreative

All done! Thanks for your participation 


\section{APPENDIX C}

- Survey Questions (Study III(A)) 


\section{BELIEVE IT OR NOT - The Sunday Star-Times beliefs survey}

YOUR BELIEFS (part 1)

Many of the questions in this survey ask you to read a statement and then indicate your response to those statements. Please read each statement, and select a response that best reflects your opinion.

Some sections require that you answer at least some (and in some cases, all) of the questions before you can continue. This is so that we have enough information to reliably report results.

* Some of the questions that follow refer to paranormal phenomena - things that cannot be explained and/or proven by current scientific methods. Select the option that best indicates how much you agree or disagree with each item. There are no right or wrong answers. This is a sample of your own beliefs and attitudes:

The soul continues to exist though the body may die.
Some individuals are able to levitate (lift) objects through mental forces.
Black magic really exists.
Yaur mind or soul can leave your body and travel (astral projection).
Humban babomiterally Do exist.
Astrology is a way to accurately predict the future.
There is a devil.
Psychokinesis, the movement of objects through psychic powers, does exist.
Witches do exist.
All animate and inanimate objects have a life-force/essence that binds body and
spirit together.
If you break a mirror, you will have bad luck.
During altered states, such as sleep or trances, the spirit can leave the body.
The Loch Ness monster of Scotland exists.

Yellow = Mavi belief

Purple = Endorsement of scientific theory.

The rest come from the Paranormal Belief Scale (Revised) Tobacyk, 1988 


\section{BELIEVE IT OR NOT - The Sunday Star-Times beliefs survey}

YOUR BELIEFS (part 2)

* Some of the questions that follow refer to paranormal phenomena - things that cannot be explained and/or proven by current scientific methods. Select the option that best indicates how much you agree or disagree with each item. There are no right or wrong answers. This is a sample of your own beliefs and attitudes:

The horoscope accurately tells a person's future.
I believe in God.
Humanity as we now know it evolved over a long period of time from more primitive
organisms.
A person's thoughts can influence the movement of a physical object.
Through the use of formulas and incantations, it is possible to cast spells on
persons.
The number "13" is particularly unlucky (or particularly lucky)
Reincarnation does occur.
There is life on other planets.
drive the expansion of the universe today.
Some psychics can accurately predict the future.
There is a heaven and/or a hell.
Mind reading is not possible.
There are actual cases of witcheraft.
It is possible to communicate with the dead.
Some people have an unexplained ability to predict the future.
Some numbers, and/or sequences of numbers are particularly lucky/unlucky.

$$
\begin{aligned}
& \text { Purple-scientific endorserent again. } \\
& \text { yellow - God knows. One of Marc's I think. } \\
& \text { Leave it out when doing analyses } \\
& \text { with the PBS. }
\end{aligned}
$$$$
\text { The rest are PBS items. }
$$ 
BELIEVE IT OR NOT - The Sunday Star-Times beliefs survey

Have you ever had a 'paranormal' experience - one that can't be explained scientifically, or 'proven' in ways that a scientist would accept?

Yes

No

If yes, please describe the experience briefly: 


\section{BELIEVE IT OR NOT - The Sunday Star-Times beliefs survey}

LUCK

* How lucky are you?

In general I consider myself:

In general, I am a lucky person: that is, I tend to experience lucky
breaks and be in the right place at the right time

\section{LOTTO!}

How frequently do you buy a
Lotto ticket?

\section{Your numbers}

If you do buy Lotto tickets, do you regularly use the same numbers?

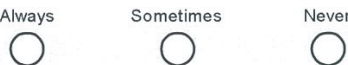

Numbers and outlets

If you do buy Lotto tickets, do you believe that some numbers are luckier than others?

If you do buy Lotto tickets, do you believe that some Lotto outlets are luckier than

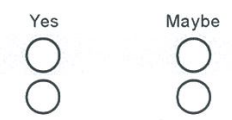

$\bigcirc^{\text {Maybe }}$

$\bigodot^{\text {No }}$

others?

Imagine that you're picking 6 numbers for regular Lotto (e.g., not including the Powerball number). Select six numbers from the options below:

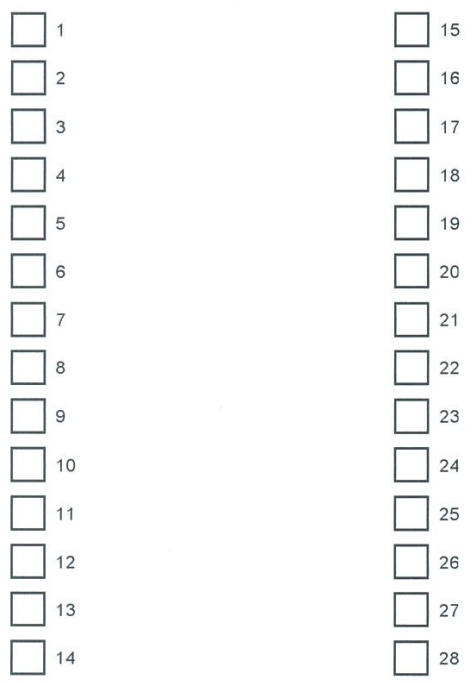

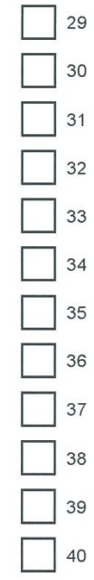




\section{BELIEVE IT OR NOT - The Sunday Star-Times beliefs survey}

$\begin{array}{ll}\text {...and the Powerball number? } & \bigcirc_{6} \\ \bigcirc_{2} & \bigcirc_{7} \\ \bigcirc_{3} & \bigcirc_{8} \\ \bigcirc_{4} & \bigcirc_{9} \\ \bigcirc_{5} & \bigcirc_{10}\end{array}$


BELIEVE IT OR NOT - The Sunday Star-Times beliefs survey

SPIRITUALITY AND RELIGION

* Consider the following questions

Do you consider yourself to be a spiritual person?

Do you consider yourself to be a religious person?

Do you have a particular religious or spiritual faith'?

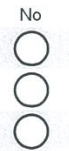

$\bigcirc$

If you do have a religious or spiritual 'faith', WHICH of the following describes what you believe (tick a box, and indicate more detail if appropriate):
Christianity
Islam
Hinduism
Judaism
Buddhism
other

Denomination/details: 
BELIEVE IT OR NOT - The Sunday Star-Times beliefs survey

* The following questions relate primarily to Christianity. Even if you are not a Christian, you can still answer the questions if you wish. Please read each of the statements below, and indicate the extent to which you agree with each one.

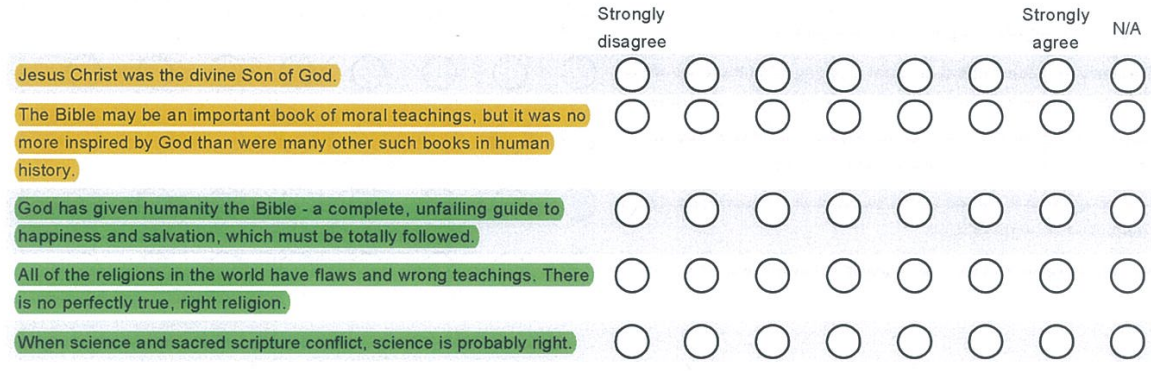

$$
\begin{aligned}
& \text { Yellow }=\text { Christian orthodoxy scale } \\
& \text { Green }=\text { Christian fundamentalism scalc. }
\end{aligned}
$$




\section{BELIEVE IT OR NOT - The Sunday Star-Times beliefs survey}

* The following questions relate primarily to Judaism. Please read each of the statements below, and indicate the extent to which you agree with each one.

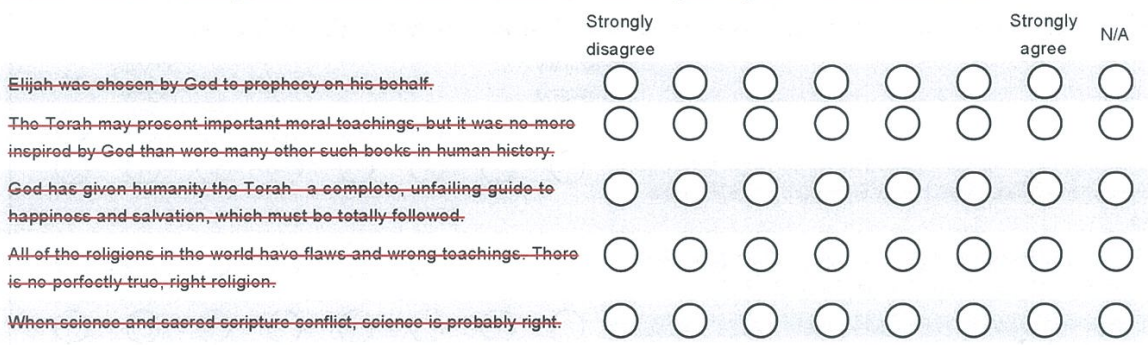

Not in the datafile. 


\section{BELIEVE IT OR NOT - The Sunday Star-Times beliefs survey}

*The following questions relate primarily to Islam. Please read each of the statements below, and indicate the extent to which you agree with each one.

$$
\text { Strongly Strongly N/A }
$$

1

There is no-other-God-but-Allah.

Mohammad is God's prophot.

Allah has-givon humanity tho Koran a-comploto, unfailing-guidoto happinoss and salvation, which must bototally followod.

000

$0 \bigcirc \bigcirc 0$

$0 \bigcirc \bigcirc \bigcirc \bigcirc \bigcirc \bigcirc$

All of tho roligions in tho world havo flaws and wrong toachings. Thoro

0000

0000

Whon scionce and sacrod scripture confflict, scionce is probably-ight

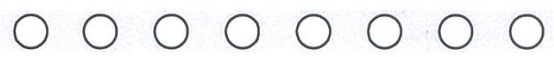

Not in the data file. 


\section{BELIEVE IT OR NOT - The Sunday Star-Times beliefs survey}

\section{Congregations and organisations}

Do you belong to a religious/spiritual congregation or organisation?

$\bigcirc^{\text {No }}$

If yes, please describe briefly:

Practice

If you belong to a congregation or

organisation, how frequently do you

meet with other members?

Daily Weekly Fortnightly Monthly $\begin{gathered}\text { Every couple Yearly Never } \\ \text { of months }\end{gathered}$

How regularly do you read scripture, or

important books associated with your

belief?

How regularly do you pray (or meditate

upon your faith)?

$\bigcirc 000000$

$\bigcirc \bigcirc \quad \bigcirc \quad \bigcirc \quad 0 \quad 0 \quad 0$

$\begin{array}{llllll}0 & 0 & 0 & 0 & 0 & 0\end{array}$ 


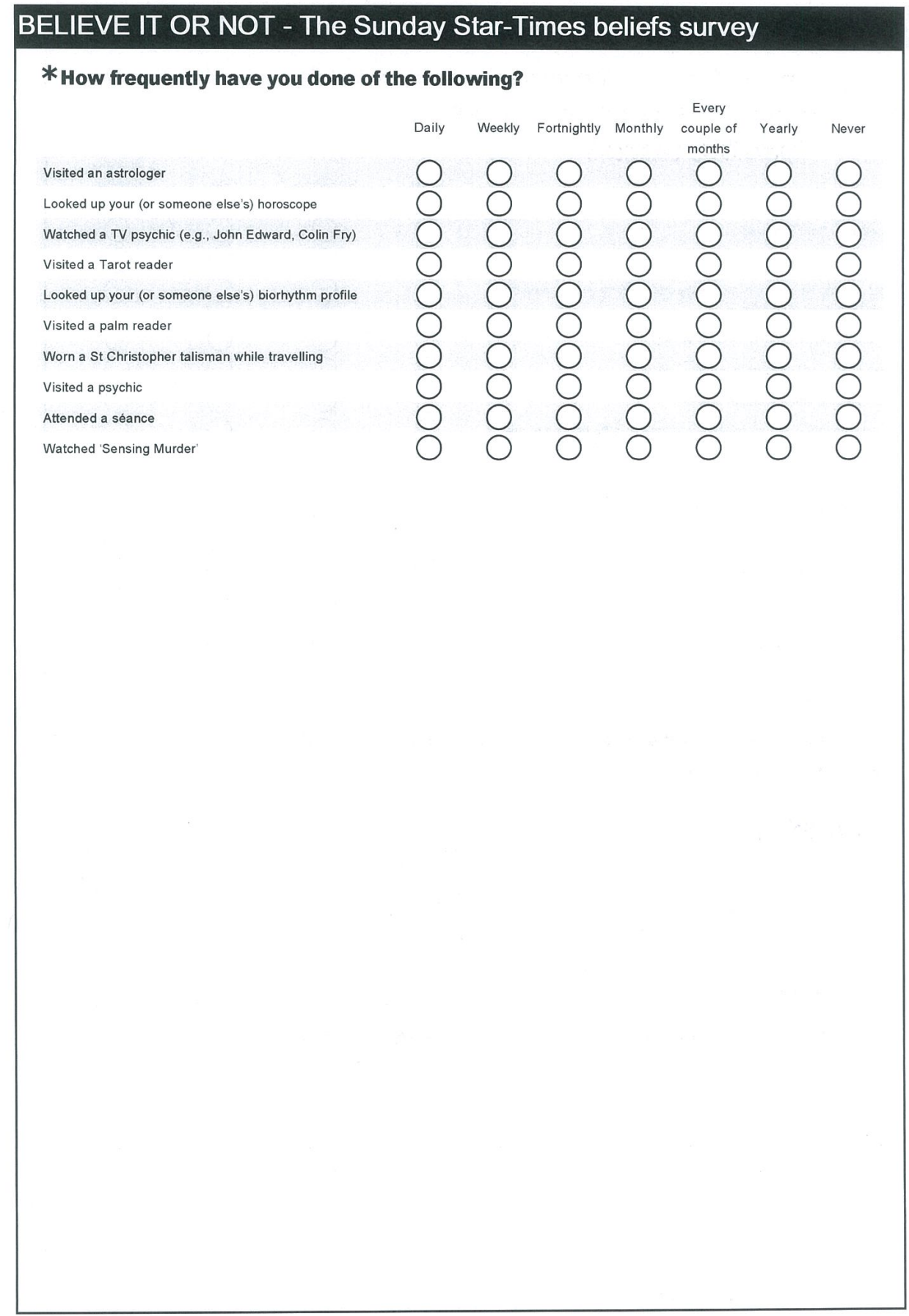




\section{BELIEVE IT OR NOT - The Sunday Star-Times beliefs survey}

The following actions are considered by some people to be lucky. How often have you performed these actions (even if only out of habit)?

Superstitious behaviours

"Knocked" or "touched" wood

Tossed spilt salt over your shoulder

Taken something into an exam, or important sports event, something that is important or significant

Avoided sitting on a table used for eating

Walked around a ladder rather than under it

Thrown a coin into a fountain or well

Actively thought about something you really want to happen or are looking forward to

Wished on a falling star

Avoided putting a hat on a table used for eating

Carried a rabbit's foot

Crossed your fingers for luck

Changed direction while walking to AVOID having a black cat cross your path

Changed direction while walking to ENSURE having a black cat cross your path

Looked for or picked a four-leaf clover

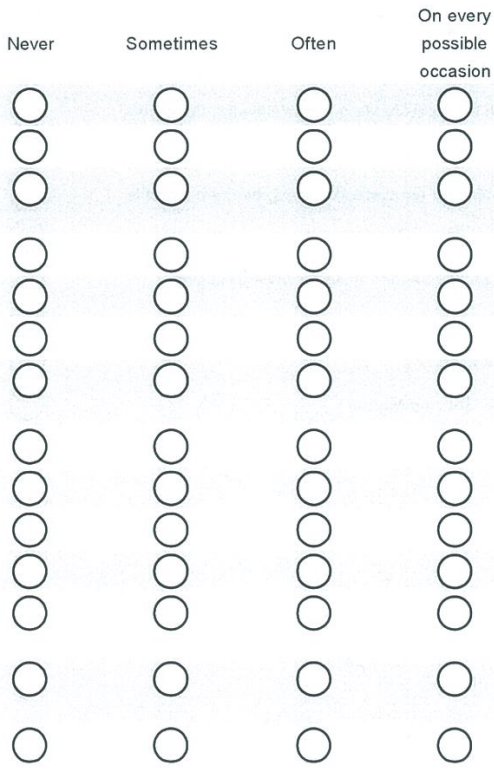

Some people think the following events can be a cause of a bad luck. Please indicate how strongly you agree.

\section{Superstitious beliefs}

It is bad luck to break a mirror

It is bad luck if a fantail comes into your house

It is bad luck to look at the new moon through glass

It is bad luck to leave chopsticks standing up in a bowl of food

It is bad luck to have 13 people at a table

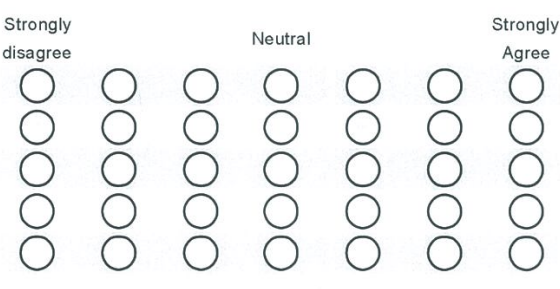

Are there any other things that you do, that you might consider superstitious? 


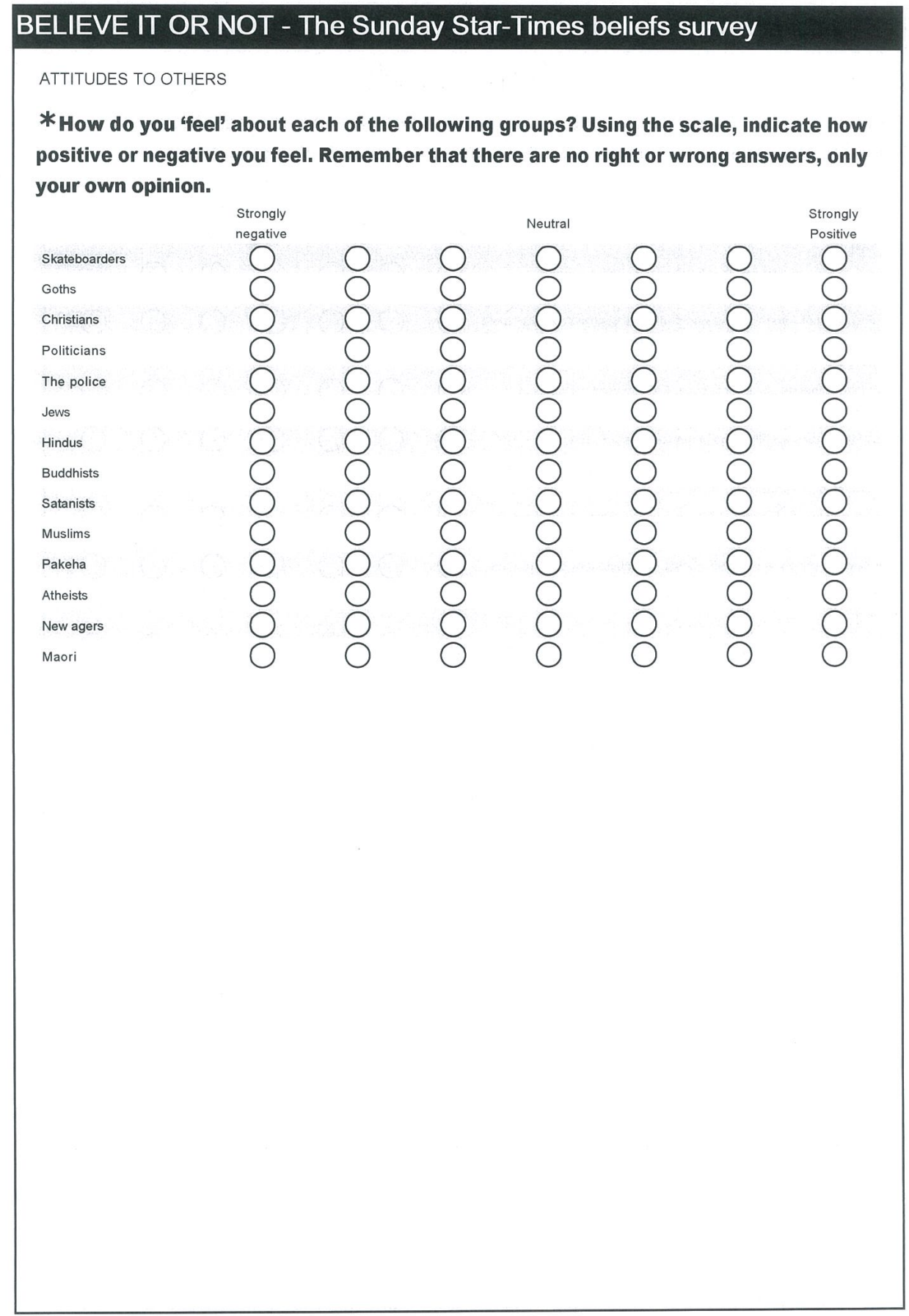




\title{
BELIEVE IT OR NOT - The Sunday Star-Times beliefs survey
}

\author{
'CONSPIRACY' THEORIES \\ Conspiracy beliefs
}

* Below is a list of theories about the causes of important or controversial events. Please read through, and indicate how likely these are as actual explanations.

The All Blacks were deliberately poisoned before the 1995 rugby world
cup final
Princess Diana was killed by the British secret service in order to
prevent a Royal scandal
A secret cabal of American and European elite control the election of
national leaders, the world economy, and direct the course of history in
their favour
There is a deliberate political conspiracy to suppress the rights of
minorities in $\mathrm{NZ}$
NASA faked some (or all) of the moon landings for publicity
The war in Iraq has less to do with promoting democracy than it does
with controlling oil production in the East
Elvis Presley faked his own death to escape the pressures of fame, the
shame of his decline, or the unwanted attentions of the Mob
New Zealand society is constantly being manipulated by Big Business
World governments are hiding evidence that the earth has been visited
by aliens
The American government was either involved in, or knew about, the
September 11 attacks before they happened




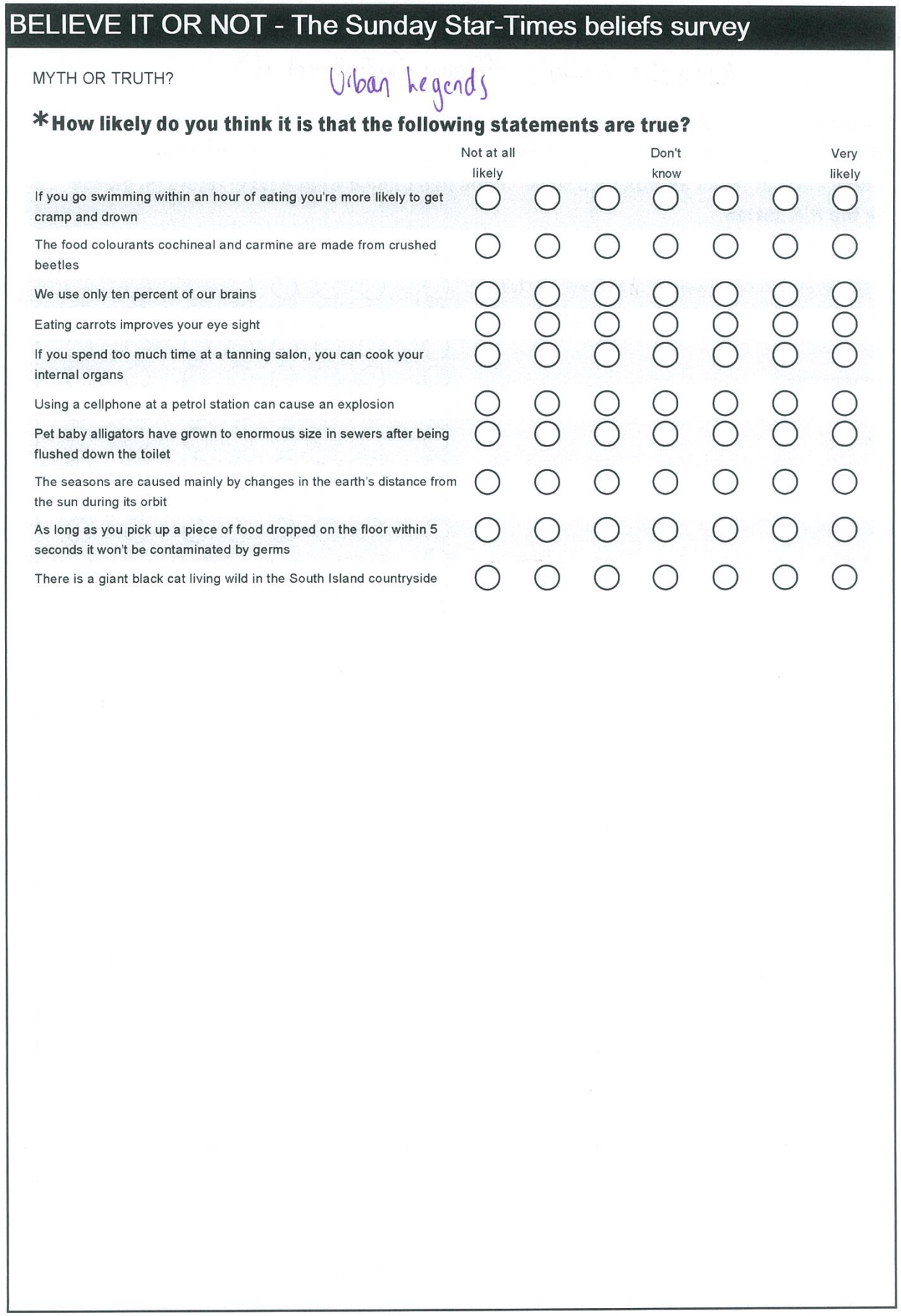




\title{
BELIEVE IT OR NOT - The Sunday Star-Times beliefs survey
}

\author{
HEALTH \\ Alternative medicine efficacy (endorsement of)
}

* Below is a list of practices that people might turn to to address illness and health problems or to ensure continuing good health. Please rate how generally effective you believe each of these approaches to be. If you don't know what a label refers to, please tick the N/A button.

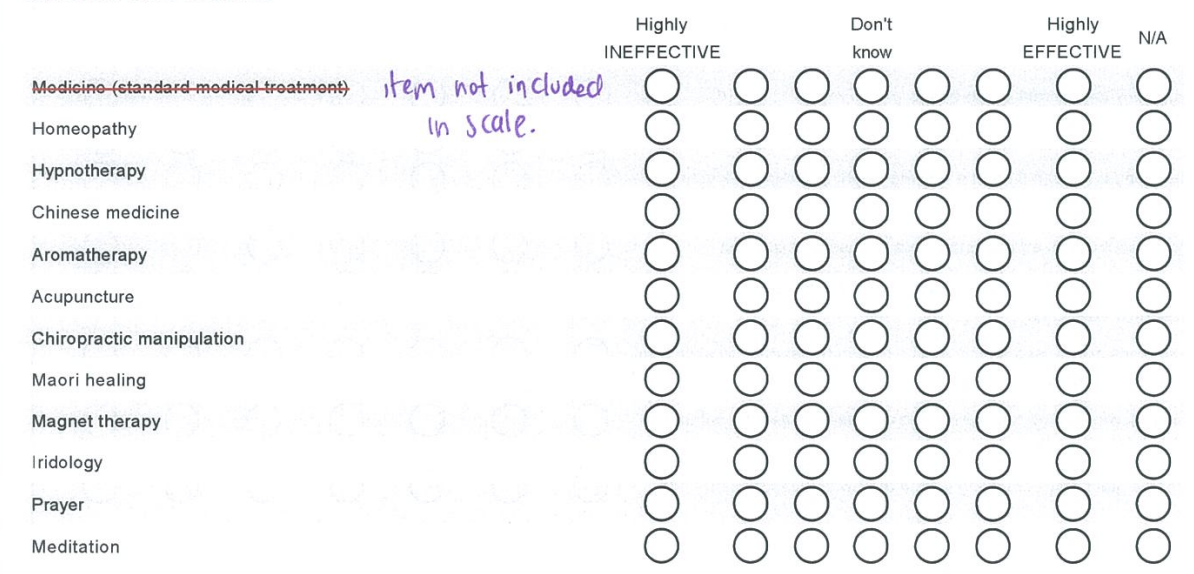




\section{BELIEVE IT OR NOT - The Sunday Star-Times beliefs survey}

Feelings about yourself and society

Please indicate your response using the scale provided.

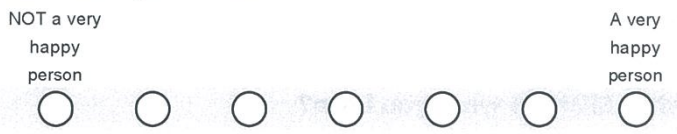

In general I consider myself:

* Please indicate your response using the scale provided.

Compared to most people I know, I consider myself:

* Below are descriptions of two 'types' of person. Read both, and indicate how well they describe you.

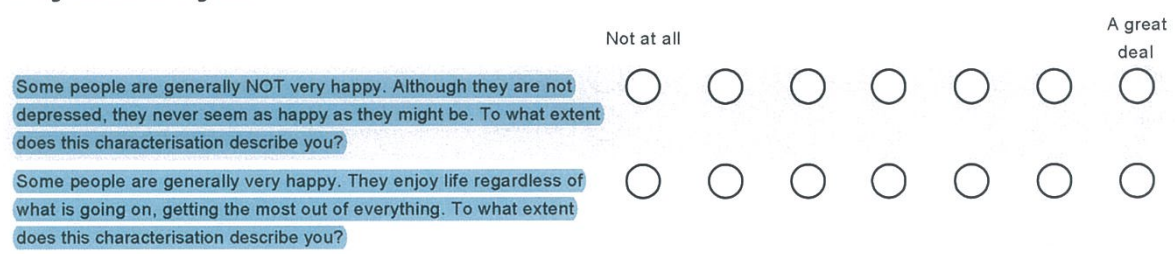

does this characterisation describe you?

* Below is a list of statements. Read each one and then indicate how much you agree (or disagree) with each using the scale provided.

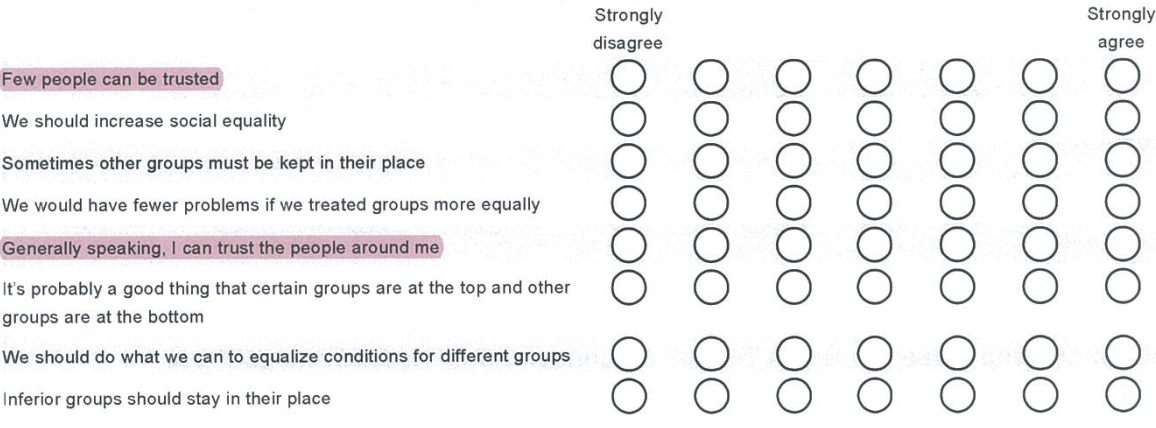

Blue: questions from Lyubomirsty et al's

Happiness scale.

Pink: items measuring distrust

The rest are items from the SDO scale. Will ark Marc

to provide a citation

for this. 
BELIEVE IT OR NOT - The Sunday Star-Times beliefs survey

The following data will help us look at differences in beliefs between different age-groups, parts of the country, or even star signs. This data will never be connected to your name.

Age in years:

In what MONTH were you born?

Select the appropriate answer from the drop down menu

What is your star sign?

aries

SCORPIO

Otaurus

Sagittarius

Gemin

CAPRICORN

Cancer

aquarius

LEO

Pisces

VIRGo

Don't know

LIBRA

* Your gender:

$\bigcirc$ Female $\bigcirc$ male

If an election were to be held today, what political party would get your party vote?

Sometimes people use the terms 'left-wing' or 'right-wing' to describe political viewpoints.

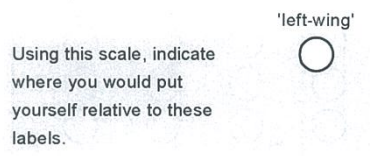
viewpoints.

Using this scale, indicate
where you would put
yourself relative to these
labels.




\section{BELIEVE IT OR NOT - The Sunday Star-Times beliefs survey}

\section{In what country were you born?}
New Zealand
China (People's Republic of)
Samoa
Australia
Japan
Cook Islands
United Kingdom
India
Other (please specify)

Which ethnic group do you belong to? Please choose one or more:

$\square$ New Zealand European/Pakeha

$\square$ Other European

$\square$ NZ Maori

Cook Island Maori

$\square$ Other Pacific Islands

$\square$ Samoan

\section{Other (please specify)}

\section{Which of the following best describes your education?}

$\square$ Left school before or during 5 th form

Completed 5 th form, then left school

$\square$ One or more years of study towards a qualification at a

$\square$ Left school before or during 7 th form polytechnic or university

Completed 7 th form

$\square$ Completed Bachelors degree / Trade Certificate / Advanced Trade Certificate

$\square$ Completed a Postgraduate degree

\section{What best describes your employment status?}

Currently in paid employment

Currently in volunteer employment

Not currently employed

If you are currently employed (either for money or as a volunteer), how many hours per week does your employment involve?

Which of the following best describes the nature of your work?

Professional or managerial

Clerical/sales/service worker

Technical, craftsman or skilled trades

Manual worker/semi-skilled/labourer

Not currently employed

What is your occupation? 


\section{BELIEVE IT OR NOT - The Sunday Star-Times beliefs survey}

Roughly speaking, which of the options describes your annual income (BEFORE tax)?

Up to $\$ 20,000$ a year ( $\$ 384$ a week)

$\$ 20,001$ to $\$ 40,000$ a year ( $\$ 385$ - $\$ 769$ a week)

$\$ 40,001-\$ 60,000$ a year $(\$ 770-\$ 1153$ a week)

$\bigcirc \$ 60,001-\$ 100,000$ a year ( $\$ 1154$ - $\$ 1923$ a week)

$\$ 100,001$ - $\$ 150,000$ a year ( $\$ 1924$ - $\$ 2884$ a week)

More than $\$ 150,000$ a year ( $\$ 2885$ or more a week)

\section{What part of the country do you live in?}

Auckland

Christchurch

Wellington

Other South Island city

Other North Island city

A town in the South Island

A town in the North Island

A smaller centre or rural area in the South Island

A smaller centre or rural area in the North Island 


\section{BELIEVE IT OR NOT - The Sunday Star-Times beliefs survey}

If you would like to be entered into the draw to win one of two Vodafone Blackberrys, please enter your contact details below. Please note, this information is stored separately from your answers, and cannot be linked back to it.

To enter the draw, complete the sections below.

Name

Street Address

Suburb

City

Post Code

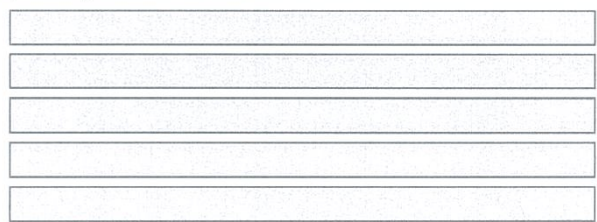




\section{BELIEVE IT OR NOT - The Sunday Star-Times beliefs survey}

Thank you for completing this survey!

We hope that you have enjoyed answering this survey, and thinking about some things you might otherwise not think about. The results will be reported in the Sunday Star Times over the next few weeks, so be sure to check back and see how typical your answers are! 


\section{APPENDIX D}

- Information Sheet for Participants (Study III(B))

- Debrief for Participants (Study III(B))

- Survey Questions (Study III(B)) 


\section{Lottery Study Information Sheet \\ (RM \#0000024667)}

Marco Zdrenka

PhD Student

Marco.Zdrenka@vuw.ac.nz
Marc Wilson

Professor of Psychology

Marc.Wilson@vuw.ac.nz

(04) 4635225

\section{What is the purpose of this research?}

- This research tests for luckiness in the lottery, or in other words, whether some people can better predict numbers in an upcoming lotto draw than others. This follows on from previous research where some types of people (e.g. people with certain personality traits) have been shown to perform better than others when asked to predict a random future outcome (milliseconds in the future). It also attempts to answer the age-old anecdotal claim that some people are just luckier than others and "always" win lotto.

\section{Who is conducting the research?}

- This research is being conducted by Marco Zdrenka, a PhD student at Victoria University of Wellington, and is supervised by Prof Marc Wilson of the School of Psychology (Marc.Wilson@vuw.ac.nz). This study has been approved by the School of Psychology Human Ethics Committee under the delegated authority of the Victoria University of Wellington Human Ethics Committee (\#0000024667).

\section{What is involved if you agree to participate?}

- You must be at least 18 years of age to participate in this study.

- If you agree to participate in this study, you will complete a personality-based survey of your views, experiences, and beliefs (this will take approximately 30 minutes in total).

- You will tell us (online) the numbers you guess for each Saturday draw for 8 weeks beginning 7 October 2017. While it is ideal that you also purchase a ticket with these numbers, it is not required.

- Each week that you submit your lotto numbers, you will be entered into a random draw for that week. Each draw will reward two entrants with a \$20 gift voucher each (you can choose from either an MTA voucher, Grocery voucher, or Movie voucher).

- Participation is entirely voluntary. If you complete the personality-based survey, you will be consenting to participation.

- During the research you are free to withdraw at any point before the end of the 8 weeks of data collection.

\section{Privacy and Confidentiality}

- Your responses will be kept completely confidential and the only identifying information we require is your email address, to send you the link to input your lotto numbers (this will be emailed to you every Friday prior to the following Saturday draw).

- Your identifying information (i.e. email address) will also link together your survey results with the 8 weeks of data on your lotto numbers. At the end of data collection, your email address will be destroyed.

- In accordance with the requirements of some scientific journals and organisations, your deidentified data may be shared with other competent researchers, and/or used in other related studies.

- You will not be identifiable in the results or any other presentation or publication of them.

- We will keep your responses electronically (without any identifying information) indefinitely.

What happens to the information that you provide?

- The data you provide may be used in a manuscript submitted for publication in a scientific journal, thesis, or presented at a scientific conference.

\section{Consent and Withdrawal}

- By entering your email address as part of the personality-based survey, you will be consenting to participate in this study.

- Furthermore, by completing the survey and submitting your lotto numbers each week, you are providing continued consent to participate. 
- You can withdraw at any time up until the end of the 8 weeks of data collection by sending an email directly to Marco (email address at the top) requesting withdrawal. All your data will then be removed from the study.

- Alternatively, you will automatically be withdrawn from the study if you fail to provide lotto numbers for two weeks in a row (however, your data provided up until this point will still be used). 


\section{Lottery Study Debrief \\ (RM \#0000024667)}

Marco Zdrenka

PhD Student

Marco.Zdrenka@vuw.ac.nz
Marc Wilson

Professor of Psychology

Marc.Wilson@vuw.ac.nz

(04) 4635225

\section{Thank you for participating in this experiment.}

The purpose of this study was to find out whether some people are luckier than others at lotto, and if so, what types of people they are.

While we aren't expecting any one person to be far luckier than anyone else in lotto (since previous research has not found such a pattern; Watt \& Nagtegaal, 2000; Smith, Wiseman, Machin, Harris, \& Joiner, 1997), we are still interested in seeing whether people with certain personalities or differences might in general perform better than others. This is because the first study of my PhD found that some people with certain individual differences did perform better in such tasks (specifically a psi task, which stands for a completely luck-based task).

The first study of my PhD was a meta-analysis that basically summarised all of the research looking at future prediction experiments where participants played a luckbased guessing game and had to choose the answer from a limited number of options. This meta-analysis looked at 35 individual differences and found that people seemed to be luckier with the following characteristics: luck belief (the belief that luck is primarily controllable), perceptual defensiveness, openness to experience, belief in psi, extraversion, and time belief as dynamic.

Our aim in this study was to test these six individual difference measures in a real-world setting (the lottery) in an attempt to see whether these relationships could be replicated all together in a single study.

If you have any questions about this research, please contact either Marco Zdrenka (Marco.Zdrenka@vuw.ac.nz) or Marc Wilson (Marc.Wilson@vuw.ac.nz).

If there was an aspect of this research that bothered you and was not addressed here, please feel free to contact us. It may also help to talk to a friend or relative, or to give Lifeline a call on 0800543354 - they provide free 24/7 counseling and support for anyone in New Zealand.

Alternatively, if you need someone to talk to about your Gambling, please give Gambling Helpline a call on 0800654655 as they provide 24/7 support for anyone needing help with their gambling (or have concerns about someone else's gambling).

If you have questions about the ethical approval of this study, please contact Associate Professor Susan Corbett (susan.corbett@vuw.ac.nz, telephone $\mathbf{+ 6 4 4 4 6 3 5 4 8 0}$ ).

\section{Marco Zdrenka}

Email: Marco.Zdrenka@vuw.ac.nz 


\section{References}

Watt, C., \& Nagtegaal, M. (2000). Luck in action? Belief in good-luck, psi-mediated instrumental response, and games of chance. Journal of Parapsychology, 64, 3352.

Smith, M., Wiseman, R., \& Harris, P. (1997). Perceived luckiness and the UK national lottery. Proceedings of Presented Papers: The Parapsychological Association's 40 th Annual Convention, 64, 387-398.

For access to a copy of these papers, please email Marco. 
Please provide your Student ID number below (to allocate credit):

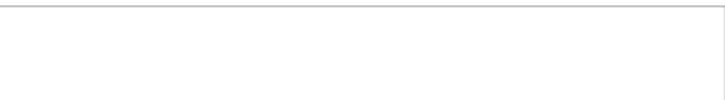

Thank you for participating in Victoria University of Wellington's (RM \#0000024667) Lotto Study.

This study will begin with asurvey to learn a little bit about you.

Following this, you'll predict lotto numbers for 8 weeks (starting Saturday October 7th until Saturday November 25th). You will receive more information via email about how this will work following the completion of this survey.

In order to award prizes to our draw winner(s) and match these responses with your lotto results, please tell us your email address below. If you do not consent to participating in this study, please do not continue. WE WILL DELETE THIS INFORMATION AFTER THE PRIZE DRAW(S) AND DATA-MATCHING. 
The following questions are designed to get a sense of how common various 'vivid' experiences are in the general population. Because these are about your experiences, there's no such thing as a right or wrong answer - the best answer is whatever makes sense to you.

Read through each statement and tell us if you've had this experience ('Yes') or not ('No')

I have occasionally had the feeling that a TV or radio broadcaster knew I was listening to
him.
I have felt that there were messages for me in the way things were arranged, like in a store
window.
Things sometimes seem to be in different places when I get home, even though no one has
been there.
I have never doubted that my dreams are the products of my own mind.
I have noticed sounds on my records that are not there at other times.
I have had the momentary feeling that someone's place has been taken by a look alike.
I have never had the feeling that certain thoughts of mine really belonged to someone else.
I have wondered whether the spirits of the dead can influence the living.
At times I perform certain little rituals to ward off negative influences.
I have felt that I might cause something to happen just by thinking too much about it.
At times, I have felt that a professor's lecture was meant especially for me.
I have sometimes felt that strangers were reading my mind.
If reincarnation were true, it would explain some unusual experiences I have had.
I sometimes have a feeling of gaining or losing energy when certain people look at me or
It is not possible to harm others merely by thinking bad thoughts about them.




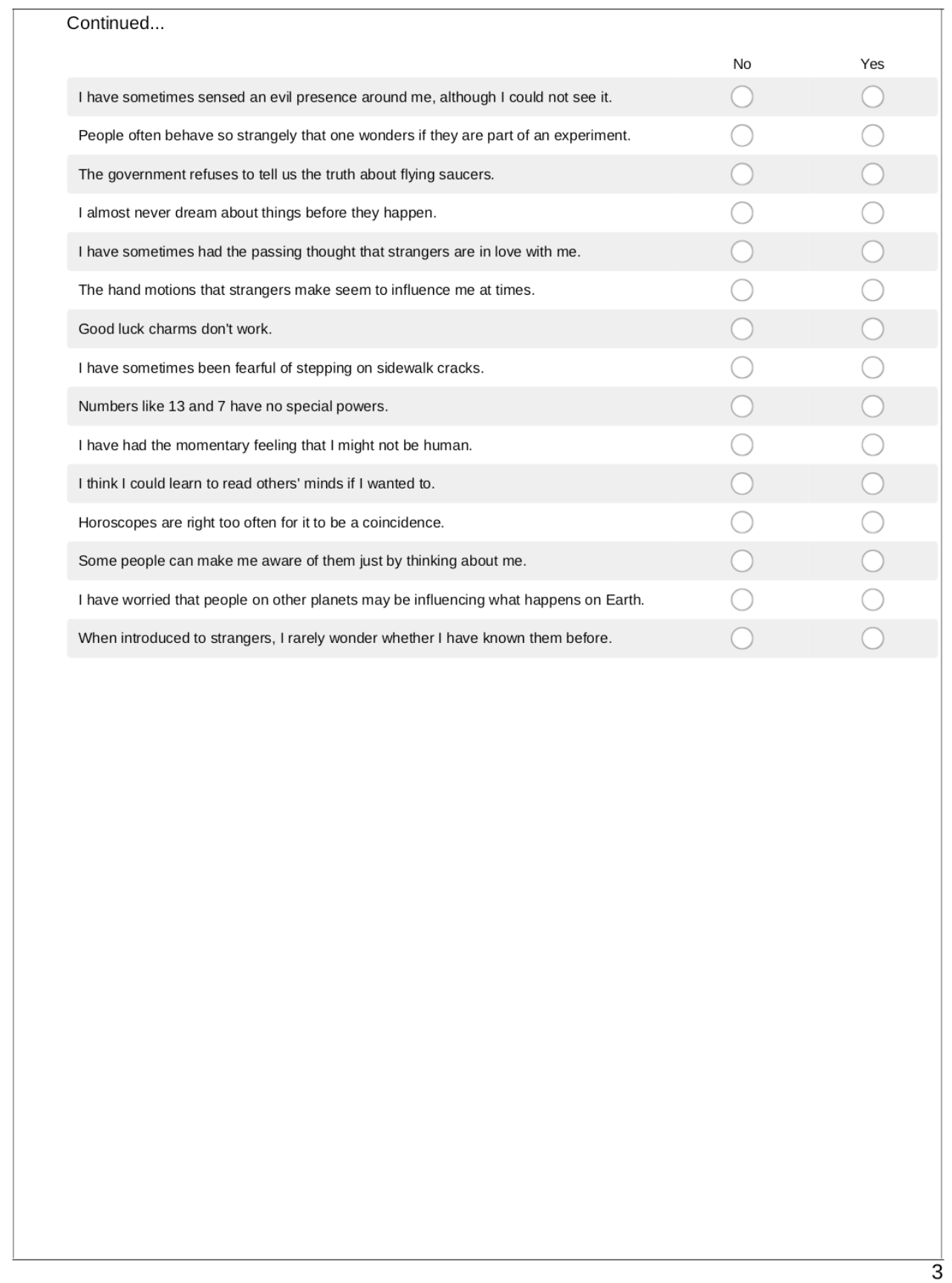


Continued..

At times I perform certain little rituals to ward off negative influences

I have experienced an altered state of consciousness in which I felt that I became cosmically enlightened

At the present time, I am very good at make-believe and imagining

I have felt that I had received special wisdom, to be communicated to the rest of humanity

I have sometimes sensed an evil presence around me, although I could not see it

My thoughts have sometimes come so quickly that I couldn't write them all down fast enough

It is sometimes possible for me to be completely immersed in nature or in art and to feel as if my whole state of consciousness has somehow temporarily been altered

Often I have a day when indoor lights seem so bright that they bother my eyes

I have experienced an altered state of consciousness which I believe utterly transformed (in a positive manner) the way I looked at myself

I think that I really know what some people mean when they talk about mystical experiences

I have gone through times when smells seemed stronger and more overwhelming than usual

I can clearly feel again in my imagination such things as: the feeling of a gentle breeze, warm sand under bare feet, the softness of fur, cool grass, the warmth of the sun and the smell of freshly cut grass

A person should try to understand their dreams and be guided by or take wamings from them

At times I somehow feel the presence of someone who is not physically there

No Yes

O

0

Q

0

For several days at a time I have had such a heightened awareness of sights and sounds that I cannot shut them out

I sometimes have a feeling of gaining or losing energy when certain people look at me or touch me

When listening to organ music or other powerful music, I sometimes feel as if I am being lifted up into the air 


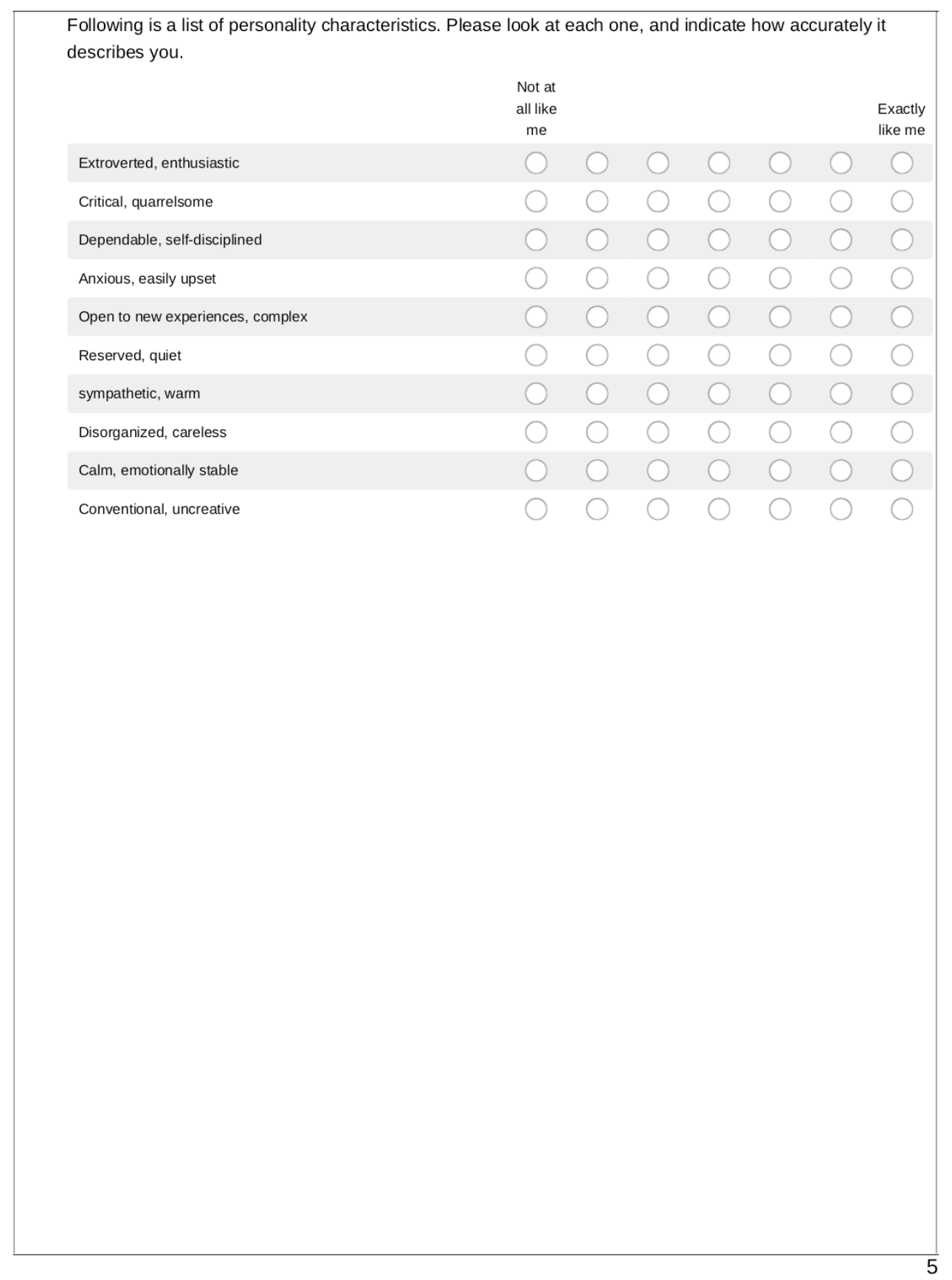


The statements below relate to the concept of 'luck'. Responses to the statements reflect a person's attitude and there are no right or wrong answers.

Please read each of the following statements and indicate how much you agree or disagree with it.

\begin{tabular}{|c|c|c|c|c|c|c|}
\hline & $\begin{array}{l}\text { Strongly } \\
\text { Disagree Disagree }\end{array}$ & $\begin{array}{l}\text { Slightly } \\
\text { Disagree }\end{array}$ & $\begin{array}{l}\text { Unsure/don't } \\
\text { Know }\end{array}$ & $\begin{array}{l}\text { Slightly } \\
\text { Agree }\end{array}$ & Agree & $\begin{array}{l}\text { Strongly } \\
\text { Agree }\end{array}$ \\
\hline \multicolumn{7}{|l|}{ Luck just happens to us } \\
\hline \multicolumn{7}{|l|}{ Lucky people are those who have good health } \\
\hline \multicolumn{7}{|l|}{ A person's luck can be improved by acting on instincts } \\
\hline \multicolumn{7}{|l|}{$\begin{array}{l}\text { Appeasement of God or a higher force can change a } \\
\text { person's luck }\end{array}$} \\
\hline \multicolumn{7}{|l|}{ Lucky events are determined by chance } \\
\hline \multicolumn{7}{|l|}{ Pessimism invites bad luck } \\
\hline \multicolumn{7}{|l|}{$\begin{array}{l}\text { Lucky people have more fulfilment in life than unlucky } \\
\text { people }\end{array}$} \\
\hline \multicolumn{7}{|l|}{ Chance determines who is lucky and who is unlucky } \\
\hline \multicolumn{7}{|l|}{ What you become in life depends on your luck } \\
\hline \multicolumn{7}{|l|}{ Unseen higher intelligences guide luck } \\
\hline \multicolumn{7}{|l|}{ A healthy family reflects a lucky life } \\
\hline \multicolumn{7}{|l|}{ Acting on lucky feelings can change one's luck } \\
\hline \multicolumn{7}{|l|}{ Supernatural forces or entities can alter a person's luck } \\
\hline \multicolumn{7}{|l|}{ All lucky and unlucky events are randomly determined } \\
\hline \multicolumn{7}{|l|}{ Being optimistic can improve a person's luck } \\
\hline \multicolumn{7}{|l|}{ The universe decides a person's luck } \\
\hline \multicolumn{7}{|l|}{ The big issues in life determine a person's luck } \\
\hline \multicolumn{7}{|l|}{ 'Higher' forces are responsible for guiding a person's luck } \\
\hline \multicolumn{7}{|l|}{ Luck just happens randomly } \\
\hline \multicolumn{7}{|l|}{ Lucky events can occur when they are desired enough } \\
\hline I consider myself to be a lucky person & & & & & & \\
\hline
\end{tabular}

Below are 25 phrases that may symbolise your sense of time. Indicate how appropriate you think each phrase is in evoking for you a satisfying image of time.

First select the five phrases that seem to you the most appropriate and put them in column (1). Then pick out the next five most appropriate phrases and put them in column (2). Continue this process until you have placed the five least appropriate phrases in your opinion in column (5). 


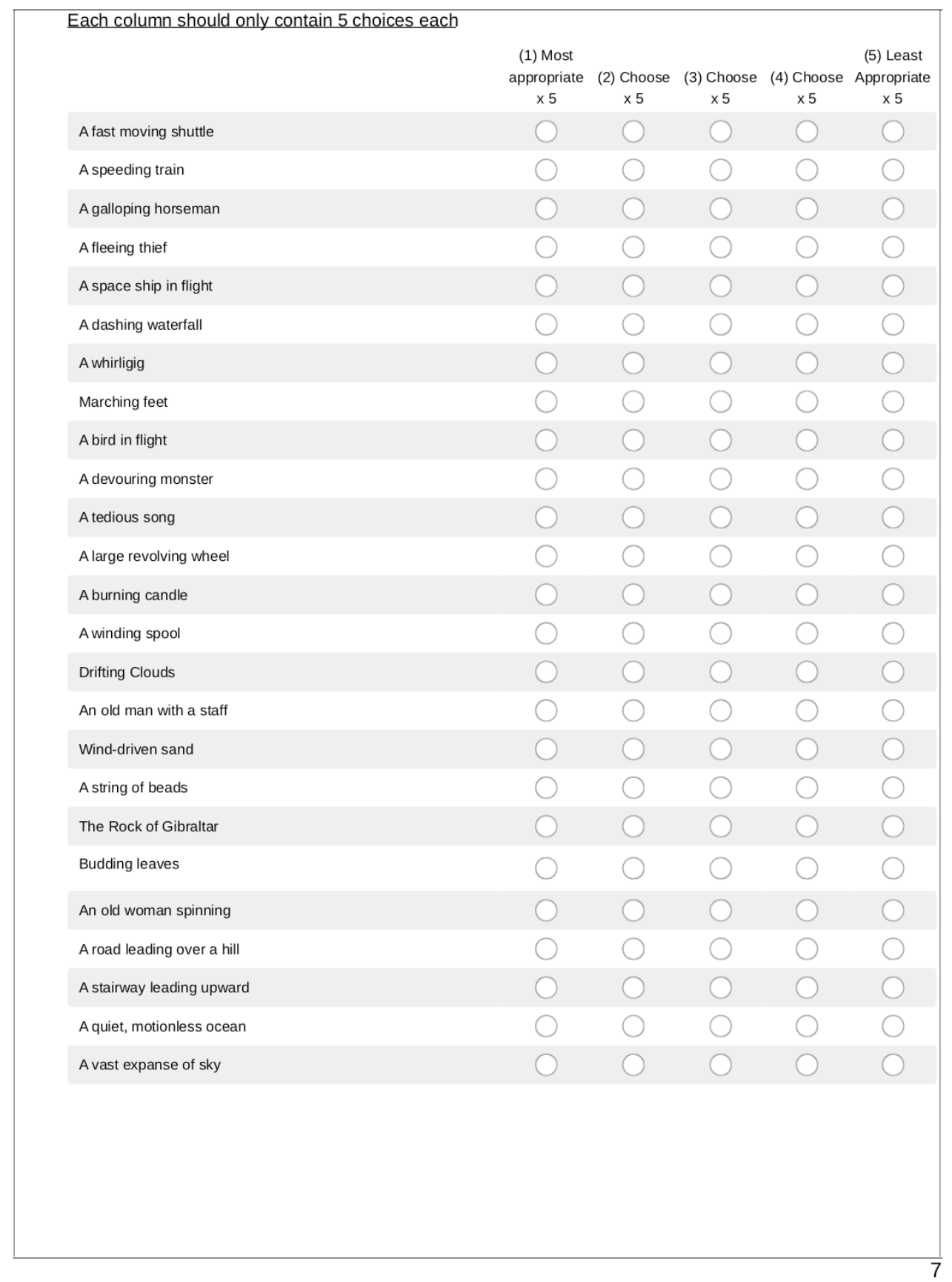


Extrasensory Perception (ESP) is the reception of information without the use of the known senses. It includes:

Telepathy: ESP of the thoughts of another person

Clairvoyance: ESP of hidden objects or distant events (also called remote viewing)

Precognition: ESP of future events

Please answer the question below.

Definitely Probably Probably Definitely Does Not Does Not Don't Know Does Does

Do you believe that ESP exists?

All done! Thanks for completing the survey section of this study.

We will be sending you an email soon with more information on the lotto draws and an overview of the study.

(You will receive this email before October 7 th)

If you have any other questions, please email lottoexperiment.vuw@gmail.com 